\title{
Top quark production at hadron colliders
}





\title{
Top quark production at hadron colliders
}

\author{
ACADEMISCH PROEFSCHRIFT
}

TER VERKRIJGING VAN DE GRAAD VAN DOCTOR

AAN DE UNIVERSITEIT VAN AMSTERDAM

OP GEZAG VAN DE RECTOR MAGNIFICUS

PROF. MR. P.F. VAN DER HEIJDEN

TEN OVERSTAAN VAN EEN DOOR HET COLLEGE VOOR PROMOTIES INGESTELDE COMMISSIE, IN HET OPENBAAR TE VERDEDIGEN

IN DE AUla DER UNIVERSITEIT

OP WOENSDAG 19 MEI 2004, TE 12:00 UUR

DOOR

\author{
Lukas Kaj Phaf
}

GEBOREN TE ROTTERDAM 
Promotores: $\quad$ Prof. dr. F. L. Linde

Prof. dr. E. L. M. P. Laenen

Co-promotor: $\quad$ Dr. ir. P. J. de Jong

Faculteit der Natuurwetenschappen, Wiskunde en Informatica

The work described in this thesis is part of the research programme of the "Nationaal Instituut voor Kernfysica en Hoge-Energie Fysica' (NIKHEF) in Amsterdam, the Netherlands. The author was financially supported by the 'Stichting voor Fundamenteel Onderzoek der Materie' (FOM). 


\section{Contents}

1 Introduction and outline $\quad 1$

2 Single-top production at Leading Order 5

2.1 Introduction . . . . . . . . . . . . . . . . . 5

2.2 Spinor helicity methods . . . . . . . . . . . . . 7

$2.3 \mathrm{~W}$-gluon fusion and $\mathrm{W}$-associated production . . . . . . . . . . 9

2.4 Flavor excitation and $s$-channel . . . . . . . . . . . . 11

2.5 Narrow width approximation . . . . . . . . . . . . . 13

2.6 Numerical studies . . . . . . . . . . . . . . . . . . . . 15

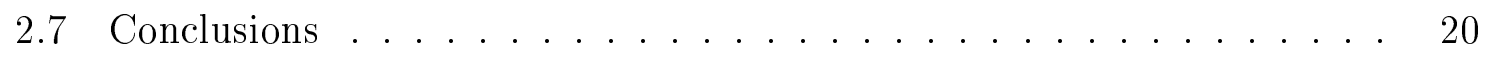

3 Massive Dipole Formalism 23

3.1 Introduction . . . . . . . . . . . . . . . . 23

3.2 Dipole formalism and factorization in singular limits . . . . . . . . . 24

3.3 Calculational technique for the integration . . . . . . . . . . 28

3.4 Dipole phase space measure in D dimensions . . . . . . . . . . . . 29

3.4.1 Phase space measure with no initial-state particles . . . . . . 30

3.4 .2 Phase space involving initial state particles . . . . . . . . . . . . 32

3.5 Dipole terms with no initial-state partons . . . . . . . . . . . 33

3.5.1 Massless final emitter, massive final spectator . . . . . . . . . 34

3.5.2 Massive final emitter, massless final spectator . . . . . . . . 37

3.5.3 Massive final emitter and massive final spectator of equal mass . 38

3.6 Dipole terms with initial-state partons . . . . . . . . . . . . 41

3.6.1 Massless initial emitter, massive final spectator . . . . . . . . 41

3.6.2 Massive final emitter, massless initial spectator . . . . . . . . 45

3.7 Conclusions . . . . . . . . . . . . . . . . . 46

4 Next to Leading Order $\quad 47$

4.1 Introduction . . . . . . . . . . . . . . . . . . 47

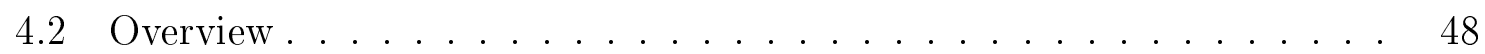


4.3 Phase space slicing . . . . . . . . . . . . . . 50

4.3.1 Phase space slicing with two cutoffs $\left(\delta_{s}, \delta_{c}\right) \ldots \ldots \ldots 51$

4.3.2 Phase space slicing with one cutoff $\left(s_{\min }\right) \ldots \ldots \ldots 55$

4.4 Massive Dipole Subtraction calculation . . . . . . . . . . . . 57

4.4.1 Calculation of the amplitudes ............. 57

4.4.2 Conversion between schemes and scheme independence . . . . . 60

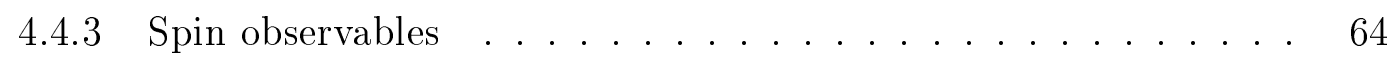

4.5 Numerical results . . . . . . . . . . . . . . . . 65

4.6 Conclusions ............................ 71

$\begin{array}{lll}5 & \text { Top pair production } & 75\end{array}$

$\begin{array}{lll}6 & \text { Experimental setup } & \mathbf{7 9}\end{array}$

6.1 The Tevatron collider . . . . . . . . . . . . . . 79

6.2 The $\mathrm{D} \varnothing$ detector ...................... 81

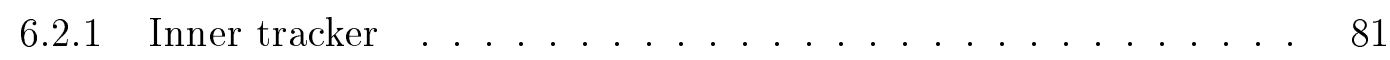

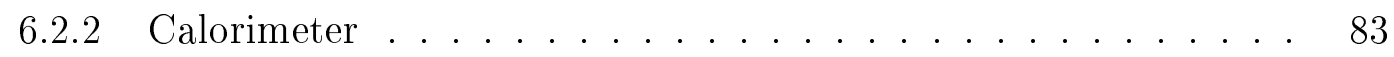

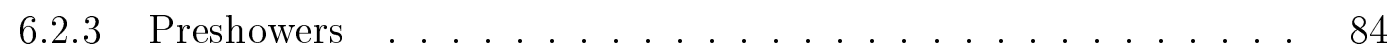

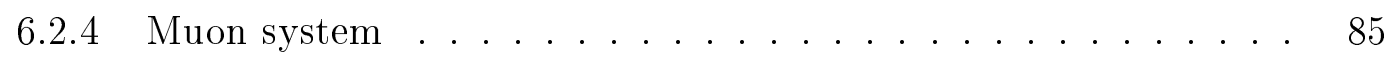

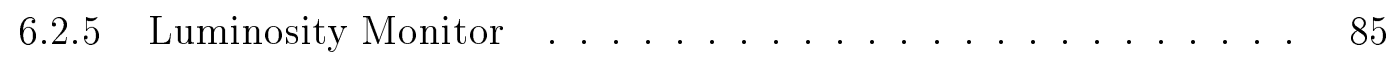

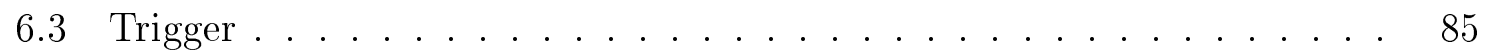

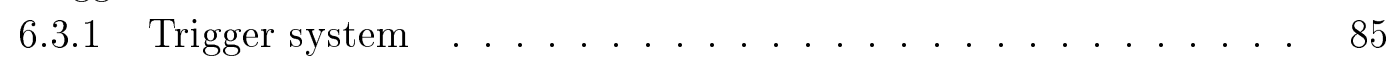

6.3 .2 Signal trigger . . . . . . . . . . . . . . . . . 87

6.3.3 Single object trigger efficiencies . . . . . . . . . . 88

6.3 .4 EM15_2JT15 efficiency . . . . . . . . . . . . . . . . . . 91

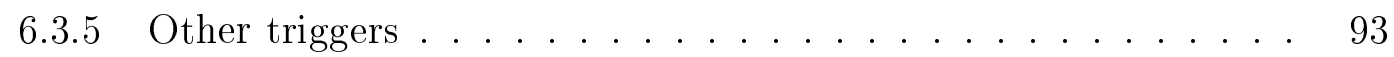

6.4 Data processing . . . . . . . . . . . . . . . . . . 93

6.5 Signal and background simulation . . . . . . . . . . . 95

$\begin{array}{lll}7 & \text { Object identification } & 97\end{array}$

7.1 Electrons . . . . . . . . . . . . . . . . . . . 97

7.1.1 Electron reconstruction and variables . . . . . . . . . . 97

7.1 .2 Sources of background . . . . . . . . . . . . . . . 99

7.1.3 Likelihood formulation . . . . . . . . . . . . . . 100

7.1.4 Likelihood performance . . . . . . . . . . . . . . 100

7.1.5 Likelihood topological dependence . . . . . . . . . . . . 108

7.1.6 Calorimeter reconstruction efficiency . . . . . . . . . . 108

7.1.7 Electron selection efficiency . . . . . . . . . . . . . 114

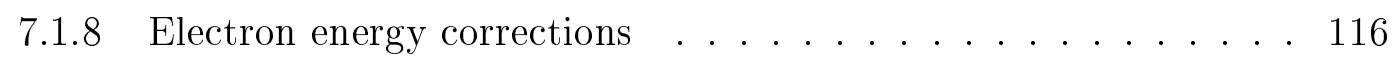

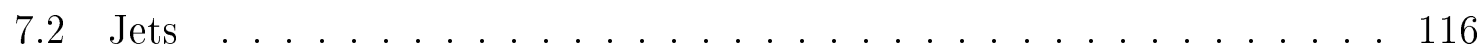


7.2 .1 Jet reconstruction . . . . . . . . . . . . . . . . 116

7.2 .2 Jet Energy Scale and resolution . . . . . . . . . . . . . 117

7.2 .3 Rejection of noise jets . . . . . . . . . . . 118

7.2 .4 Efficiency of noise rejection cuts . . . . . . . . . . 118

7.2 .5 Jet/EM separation . . . . . . . . . . . . . . . . . . . . 119

7.3 Missing transverse energy $\not_{T} \ldots \ldots \ldots \ldots \ldots$

8 Top pair cross section analysis $\quad 123$

8.1 Introduction . . . . . . . . . . . . . . . . . . 123

8.2 The $\mathrm{W}+$ jets selections . . . . . . . . . . . . . . . . . . . . . . . . . 124

8.3 QCD background . . . . . . . . . . . . . . . . 124

8.4 Berends scaling . . . . . . . . . . . . . . . . . 128

8.5 Topological selection . . . . . . . . . . . . . . . . . . . . 132

8.6 Alternative $W+$ jets background prediction . . . . . . . . . . . 135

8.7 Selection efficiency . . . . . . . . . . . . . 136

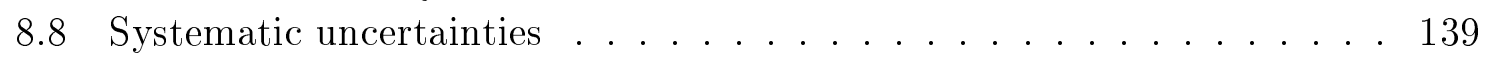

8.9 Cross section . . . . . . . . . . . . . . . . . . . 146

8.10 Combined cross section . . . . . . . . . . . . . . 147

8.11 Conclusion and outlook . . . . . . . . . . . . . . 149

$\begin{array}{lr}\text { A Leading order helicity amplitudes } & \mathbf{1 5 1}\end{array}$

A.1 Helicity amplitudes for W-gluon fusion . . . . . . . . . . . . . . 151

A.2 Helicity amplitudes for flavor excitation and the $s$-channel $\ldots \ldots$. . 156

$\begin{array}{ll}\text { B Scalar integrals } & 159\end{array}$

$\begin{array}{lr}\text { C Additional backgrounds } & \mathbf{1 6 1}\end{array}$

C.1 $W \rightarrow \tau \nu$ background . . . . . . . . . . . . . . . 161

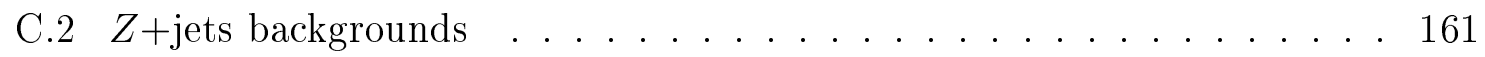

$\begin{array}{ll}\text { References } & 163\end{array}$

$\begin{array}{ll}\text { Summary } & 171\end{array}$

$\begin{array}{ll}\text { Samenvatting } & 173\end{array}$

$\begin{array}{ll}\text { Acknowledgements } & 175\end{array}$ 


\section{Chapter 1}

\section{Introduction and outline}

In this chapter we will introduce both the theoretical and experimental research described in this thesis, and mention the connection between the two.

The research in this thesis is done in the framework of the Standard Model (SM). This is a collection of gauge theories that describes all fundamental interactions known today except gravity. An important part of the SM is Quantum Chromodynamics (QCD). QCD is a relativistic quantum field theory ${ }^{1}$ based on local $S U(3)$ gauge invariance. It is the microscopic theory of the strong interaction.

Fields in the SM correspond to particles, both fermions and bosons. The heaviest fermion in the SM is the top quark. With its mass of about $175 \mathrm{GeV}$ it is much heavier than the other quarks (the mass of the next-heaviest bottom quark is about $5 \mathrm{GeV}$ ) and leptons (the heaviest lepton is the tau, which has a mass of $1.777 \mathrm{GeV}$ ).

The study of the top quark is very interesting for various reasons:

- In the SM, mass is generated by electroweak symmetry breaking. The large mass of the top quark, close to the electroweak scale, indicates that it couples strongly to the field that breaks this symmetry. The top quark could thus play an important role in understanding the generation of mass in the SM.

- In the SM, the top mass, together with the $W$ mass, constrains the Higgs mass. Combined with a direct measurement of the Higgs mass this can be used as a consistency check of the SM.

- So far, only the top quark mass has been measured. Only detailed study will tell if the properties of the top quark are as described by the SM.

- The high top mass implies a weak coupling to gluons, making it very suitable for perturbative QCD precision studies.

\footnotetext{
${ }^{1}$ Excellent expositions of quantum field theory can be found in books by Mandl and Shaw [1], Peskin and Schroeder [2] and Sterman [3].
} 
- The top quark's very short lifetime (large width) ensures that its properties are not obscured by QCD hadronization. For example, its spin can be studied directly.

It is clear from this, that studies of the top quark will be among the most important at the Tevatron and the upcoming Large Hadron Collider (LHC).

The top quark was first directly observed at the Fermilab Tevatron in 1995. The discovery was made in the process where a top/antitop pair is produced through QCD interaction $^{2}$. One of the Leading Order (LO) Feynman diagrams showing this interaction is given in Figure 1.1.

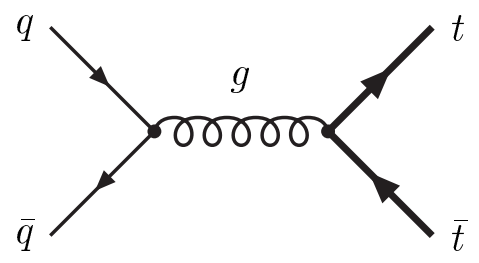

Figure 1.1: One of the LO Feynman diagrams for top pair production. The top quark line is thickened.

A top quark can also be produced through the charged-current coupling (electroweak interaction). This is called single-top production, because only one top (or antitop) quark is produced. The LO Feynman diagrams for this process are given in Figure 1.2. Single-top production has not yet been observed, due to a lower cross section and much higher backgrounds for this process.

This measurement has importance because the charged-current top coupling might be particularly sensitive to certain signals of new physics. For example, in the SM, but not in various extensions, the top quark is $100 \%$ polarized when produced via the charged-current interaction, and this structure can be verified by looking at the decay products of the top.

The first part of this thesis will develop the theoretical description of single-top production: Chapter 2 describes a calculation of single-top production at LO which includes the decay of the top quark. Numerical results of this calculation are also given. In Chapter 3, we develop techniques which are needed for a Next to Leading Order (NLO) QCD calculation with massive particles. In Chapter 4 a fully differential NLO (in QCD) calculation of single-top production is presented ${ }^{3}$.

\footnotetext{
${ }^{2}$ In Chapter 5 theoretical and experimental work that has been done on top pair production will be discussed.

${ }^{3}$ Chapters 2,3 and 4 are based on publications $[4,5,6]$.
} 


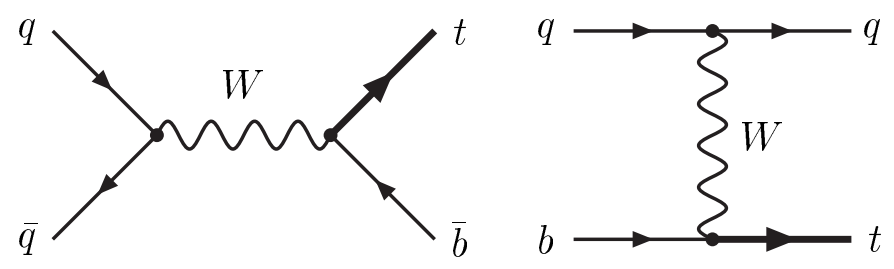

Figure 1.2: The LO Feynman diagrams for single-top production. The top quark line is thickened.

The second part of the thesis describes the measurement of a top cross section in recent data taken with the $\mathrm{D} \varnothing$ detector at the Tevatron collider. Due to the limited availability of data, a discovery of single-top production was not possible at the time of writing of this thesis. We therefore decided to do a measurement of the top pair cross section. Because many of the techniques used in this measurement are similar to the single-top cross section measurement, this will be an excellent preparation for the single-top measurement. Moreover, top pair production is an important background to single-top production. 


\section{Chapter 2}

\section{Single-top production at Leading Order}

\section{$2.1 \quad$ Introduction}

There are various partonic subprocesses that lead to the production of a single top. The ones for which we derive tree-level helicity amplitudes are the "W-gluon fusion" process

$$
u+g \rightarrow t+d+\bar{b}
$$

the "flavor excitation" process

$$
u+b \rightarrow t+d
$$

the Drell-Yan like "s-channel" process, which occurs via a virtual timelike W-boson

$$
u+\bar{d} \rightarrow t+\bar{b}
$$

and the "W-associated" production process

$$
g+b \rightarrow t+W
$$

with the $\mathrm{W}$ decaying hadronically. In reactions (2.1) and (2.2) it is understood that we may replace the $(u, d)$-quark pair by $(\bar{d}, \bar{u}),(c, s)$ and $(\bar{s}, \bar{c})$. In reaction $(2.3)$ we may replace the $(u, \bar{d})$-pair by $(c, \bar{s})$. In addition, CKM suppressed combinations may be included.

Because of its large mass, the electroweak decay of the top quark proceeds so rapidly that top bound states do not have time to form [7]. This also means that the decay 
products of the top quark are correlated with its spin. It is therefore desirable to include the semileptonic decay ${ }^{1}$ of the top quark in the amplitudes:

$$
t \rightarrow b+\bar{l}+\nu
$$

The complete tree-level amplitudes for flavor excitation and $s$-channel production can be obtained by crossing from those for

$$
0 \rightarrow b+\bar{l}+\nu+\bar{b}+\bar{u}+d .
$$

with "0" representing the vacuum. Likewise, the amplitudes for W-gluon fusion and $\mathrm{W}$-associated production are obtained by crossing from

$$
0 \rightarrow b+\bar{l}+\nu+\bar{b}+\bar{u}+d+g
$$

In this chapter we present the complete tree-level helicity amplitudes for (2.6) and (2.7). We obtain compact expressions by using spinor helicity methods. We present in addition all amplitudes for the subprocesses (2.1-2.4) in the narrow top width approximation $[8,9]$. This allows us to check the quality of this approximation against the full calculation, for which only a few of the diagrams actually involve a top quark ${ }^{2}$.

Although the helicity amplitudes for (2.1-2.4), including top decay, may be obtained as well as Fortran code from the program Madgraph [11], we believe that our analytical results are valuable for a number of reasons. First, analytical expressions can offer additional insights, e.g. in the phase space structure of the cross section near the top mass pole. Second, they lead to even more compact computer codes by allowing numerical crossing, and third, they allow evaluation in terms of spinor products, and in different computer languages (we use $\mathrm{C}++$ ). Finally, our results constitute a nontrivial check on this useful program. In this context we also mention the program Onetop [12], and the general purpose programs Pythia [13] and Herwig [14] which can be used as event generators for single-top production.

In order to test these amplitudes numerically, we study various distributions in momentum and angle of some of the final state particles for each process separately. We limit ourselves here to the subprocesses (2.1-2.3), because process (2.4) is negligible $[15,16]$ at the Tevatron. We note that whether a subprocess is a leading order contribution or a higher order correction to another, depends on the definition of the final state. Thus, for sufficiently inclusive quantities the W-gluon fusion and flavor excitation processes are not independent: a part of the former is then in fact a higher order QCD correction to the latter, and must be mass factorized [17]. A similar argument applies to W-gluon fusion and the $s$-channel process in the case where the gluon

\footnotetext{
${ }^{1}$ Single-top production with hadronic top decay suffers from large QCD backgrounds.

${ }^{2} \mathrm{~A}$ study of finite width effects in $t \bar{t}$ production is given in [10].
} 
couples to the $u$ - $d$-quark line, and the signal is defined to be inclusive with respect to the presence of the light quark jet. Furthermore, whether bottom quarks are part of the initial state is a choice of factorization scheme. We adopt a five-flavor scheme and include bottom quark parton distribution functions. In this chapter we wish to examine some characteristics of each process individually, rather than perform a comprehensive phenomenological study involving combinations of these processes and their backgrounds, as such studies already exist in the literature [16, 18]. We therefore focus on exclusive quantities, e.g. we require exactly three jets or exactly two jets.

Our conventions for spinor helicity methods are listed in Section 2.2. Sections 2.3 and 2.4, together with Appendix A, contain the helicity amplitudes for processes (2.6) and (2.7). In Section 2.5 we discuss the narrow top width approximation, while the results of our numerical studies can be found in Section 2.6. We conclude in Section 2.7.

\subsection{Spinor helicity methods}

To compute the amplitudes for (2.6) and (2.7) we use spinor helicity methods [19, 20, $21,22,23]$. We limit ourselves here to listing our conventions; for reviews of spinor helicity methods see e.g. [24, 25].

With spinor helicity methods we can express scattering amplitudes in terms of massless Weyl spinors of helicity $\pm \frac{1}{2}$

$$
u(p, \pm)=v(p, \mp)=|p \pm\rangle, \quad \bar{u}(p, \pm)=\bar{v}(p, \mp)=\langle p \pm| .
$$

External fermion states are directly expressed in terms of these. Our convention is to take all particles outgoing. For example an outgoing massless fermion with positive helicity is denoted by $\langle p+|$, while an outgoing massless antifermion with positive helicity is denoted by $|p-\rangle$. The gluon polarization vectors, of helicity \pm 1 , may be written as

$$
\varepsilon_{\mu}^{+}(k, q)=\frac{\left\langle q-\left|\gamma_{\mu}\right| k-\right\rangle}{\sqrt{2}\langle q k\rangle}, \quad \varepsilon_{\mu}^{-}(k, q)=\frac{\left\langle q+\left|\gamma_{\mu}\right| k+\right\rangle}{\sqrt{2}[k q]} .
$$

We have used the customary short-hand notation:

$$
\langle i j\rangle=\left\langle p_{i}-\mid p_{j}+\right\rangle, \quad[i j]=\left\langle p_{i}+\mid p_{j}-\right\rangle .
$$

In (2.9) $k$ is the gluon momentum and $q$ an arbitrary light-like "reference momentum". The dependence on the choice of $q$ drops out in gauge-invariant amplitudes. We shall also employ the abbreviations

$$
\begin{aligned}
\langle i-|k+l| j-\rangle & =\langle i k\rangle[k j]+\langle i l\rangle[l j] \\
s_{i j \ldots k} & =\left(p_{i}+p_{j}+\ldots+p_{k}\right)^{2}
\end{aligned}
$$


with all momenta null-vectors.

To treat the massive top quark within the framework of spinor helicity methods, we use the extension to massive fermions [20, 26, 27, 28]. Even though helicity is not a conserved quantum number for a massive particle, a massive positive-energy spinor satisfying the Dirac equation has a two-fold degeneracy (e.g. labelled by a spincomponent quantized along some axis). With slight abuse of notation we label these two states by "+" and "-_". Let $p$ be a four-vector with $p^{2}=m^{2}$ and $p_{0}>0$, and let $q$ be an arbitrary null vector with $q_{0}>0$. We define

$$
\begin{array}{ll}
u(p,+)=\frac{1}{\sqrt{2 p q}}(\not p+m)|q-\rangle, & v(p,+)=\frac{1}{\sqrt{2 p q}}(\not p-m)|q-\rangle, \\
u(p,-)=\frac{1}{\sqrt{2 p q}}(\not p+m)|q+\rangle, & v(p,-)=\frac{1}{\sqrt{2 p q}}(\not p-m)|q+\rangle .
\end{array}
$$

For the conjugate spinors we have

$$
\begin{array}{ll}
\bar{u}(p,+)=\frac{1}{\sqrt{2 p q}}\langle q-|(\not p+m), & \bar{v}(p,+)=\frac{1}{\sqrt{2 p q}}\langle q-|(\not p-m), \\
\bar{u}(p,-)=\frac{1}{\sqrt{2 p q}}\langle q+|(\not p+m), & \bar{v}(p,-)=\frac{1}{\sqrt{2 p q}}\langle q+|(\not p-m) .
\end{array}
$$

It is easy to check that for these spinors the Dirac equations, orthogonality and completeness relations hold. The dependency on the arbitrary reference momentum $q$ drops out in the final answer.

Given two four-vectors $p$ and $q$, the spinor product $\langle p q\rangle$ is calculated as follows: If $p_{t}>0$ and $q_{t}>0$,

$$
\langle p q\rangle=\frac{1}{\sqrt{p_{+} q_{+}}}\left(p_{\perp} q_{+}-p_{+} q_{\perp}\right) .
$$

Here the light-cone coordinates $p_{+}=p_{t}+p_{z}$ and $p_{\perp}=p_{x}+i p_{y}$ are used. For negativeenergy four-vectors we have

$$
\begin{array}{ll}
\langle p q\rangle=-\langle(-p) q\rangle, & \text { for } p_{t}<0, \\
\langle p q\rangle=-\langle p(-q)\rangle, & \text { for } q_{t}<0 .
\end{array}
$$

The spinor product $[p q]$ is related to $\langle p q\rangle$ by

$$
[p q]=\operatorname{sign}\left(p_{t} q_{t}\right)\langle q p\rangle^{*} .
$$

A massive quark propagator may be expressed in terms of massless Weyl spinors via

$$
\frac{i \delta_{i j}}{p^{2}-m^{2}+i \epsilon}\left(p_{+}+p_{-}+m\right)
$$


where $p_{+}$and $p_{-}$are of the form

$$
\begin{aligned}
& p_{+}=\left|p_{1}+\right\rangle\left\langle p_{1}+|+\ldots+| p_{n}+\right\rangle\left\langle p_{n}+\right| \\
& p_{-}=\left|p_{1}-\right\rangle\left\langle p_{1}-|+\ldots+| p_{n}-\right\rangle\left\langle p_{n}-\right|
\end{aligned}
$$

for some nullvectors $p_{1}, \ldots, p_{n}$.

For all other vertices and propagators we use standard Feynman rules, in the conventions of [29], and the 't Hooft-Feynman $R_{\xi}$-gauge with $\xi=1$. We neglected all fermion masses except the top mass. As a consequence, neither diagrams containing a Higgs boson nor diagrams with would-be Goldstone bosons contribute.

\subsection{W-gluon fusion and $\mathrm{W}$-associated production}

In this section we present the helicity amplitudes for the process

$$
0 \rightarrow \nu\left(p_{1}\right)+\bar{l}\left(p_{2}\right)+b\left(p_{3}\right)+\bar{b}\left(p_{4}\right)+g\left(p_{5}\right)+d\left(p_{6}\right)+\bar{u}\left(p_{7}\right) .
$$

The amplitudes are calculated in tree approximation at order $O\left(g g_{w}^{4}\right)$, where $g$ denotes the strong coupling and $g_{w}$ the electroweak coupling. There are also tree diagrams of order $O\left(g^{3} g_{w}^{2}\right)$ ("QCD + Weak") contributing to (2.19). This gauge-invariant set of graphs does not contain a top quark as an intermediate state, and we do not consider it.

The results for the (W-gluon fusion) processes $u+g \rightarrow \nu+\bar{l}+b+\bar{b}+d$ and $\bar{d}+g \rightarrow \nu+\bar{l}+b+\bar{b}+\bar{u}$, as well as for the (W-associated) process $b+g \rightarrow \nu+\bar{l}+b+d+\bar{u}$, can be obtained from those of process (2.19) by crossing, under which the crossed momentum and helicity change sign. The color decomposition for the amplitude (2.19) reads

$$
A_{W g}=g T_{34}^{a} \delta_{67} A_{W g}^{(1)}+g \delta_{34} T_{67}^{a} A_{W g}^{(2)} .
$$

The partial amplitudes $A_{W g}^{(1)}$ and $A_{W g}^{(2)}$ are gauge-invariant by themselves. $A_{W g}^{(1)}$ corresponds to diagrams where the gluon couples to the $b-\bar{b}$-fermion line, whereas $A_{W g}^{(2)}$ involves the gluon coupling to the $d-\bar{u}$-fermion line. Representative Feynman diagrams for the partial amplitudes $A_{W g}^{(1)}$ and $A_{W g}^{(2)}$ are shown in Figure 2.1 and Figure 2.2, respectively. There are 21 diagrams contributing to $A_{W g}^{(1)}, 3$ of them contain a top quark. The partial amplitude $A_{W g}^{(2)}$ is made up of 24 diagrams, with 2 containing a top quark.

For the color matrices we have used the short-hand notation $T_{34}^{a}=T_{i_{3} j_{4}}^{a}$. They are normalized as

$$
\operatorname{Tr} T^{a} T^{b}=\frac{1}{2} \delta^{a b} .
$$




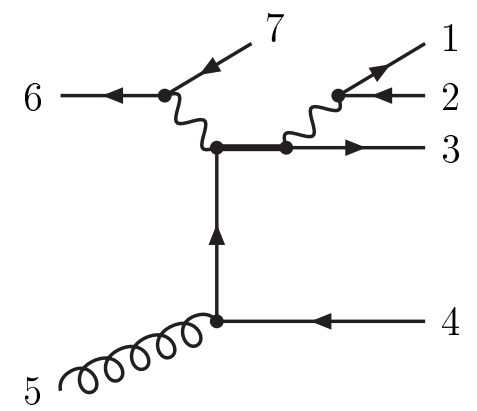

Figure 2.1: A representative Feynman diagram for single-top production contributing to the partial amplitude $A_{W g}^{(1)}$ (2.20). The top quark line is thickened.

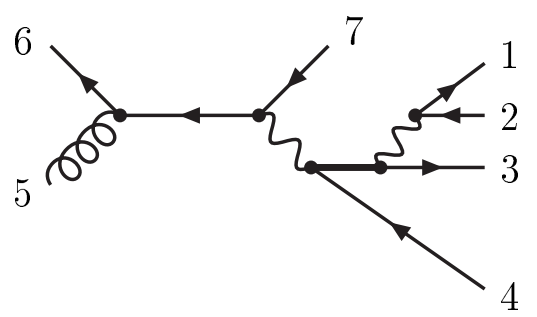

Figure 2.2: A representative Feynman diagram for single-top production contributing to the partial amplitude $A_{W g}^{(2)}$ (2.20). The top quark line is thickened.

We can decompose the two subamplitudes in (2.20) further according to their electroweak structure:

$$
\begin{aligned}
A_{W g}^{(1)}= & \frac{e^{4} V_{u d}^{*}}{2 \sin ^{2} \theta_{W}}\left(\frac{\left\|V_{t b}\right\|^{2}}{2 \sin ^{2} \theta_{W}} A_{W g}^{(1,1)}+\left(v_{d}^{\gamma} v_{b}^{\gamma}+v_{d}^{Z} v_{b}^{Z} \mathcal{P}_{Z}\left(s_{345}\right)\right) A_{W g}^{(1,2)}\right. \\
& +\left(v_{u}^{\gamma} v_{b}^{\gamma}+v_{u}^{Z} v_{b}^{Z} \mathcal{P}_{Z}\left(s_{345}\right)\right) A_{W g}^{(1,3)}+\left(v_{b}^{\gamma}-\frac{\cos \theta_{W}}{\sin \theta_{W}} v_{b}^{Z} \mathcal{P}_{Z}\left(s_{345}\right)\right) A_{W g}^{(1,4)} \\
& \left.+\left(v_{e}^{\gamma} v_{b}^{\gamma}+v_{e}^{Z} v_{b}^{Z} \mathcal{P}_{Z}\left(s_{345}\right)\right) A_{W g}^{(1,5)}+v_{\nu}^{Z} v_{b}^{Z} \mathcal{P}_{Z}\left(s_{345}\right) A_{W g}^{(1,6)}\right) \\
A_{W g}^{(2)}= & \frac{e^{4} V_{u d}^{*}}{2 \sin ^{2} \theta_{W}}\left(\frac{\left\|V_{t b}\right\|^{2}}{2 \sin ^{2} \theta_{W}} A_{W g}^{(2,1)}+\left(v_{d}^{\gamma} v_{b}^{\gamma}+v_{d}^{Z} v_{b}^{Z} \mathcal{P}_{Z}\left(s_{34}\right)\right) A_{W g}^{(2,2)}\right. \\
& +\left(v_{u}^{\gamma} v_{b}^{\gamma}+v_{u}^{Z} v_{b}^{Z} \mathcal{P}_{Z}\left(s_{34}\right)\right) A_{W g}^{(2,3)}+\left(v_{b}^{\gamma}-\frac{\cos \theta_{W}}{\sin \theta_{W}} v_{b}^{Z} \mathcal{P}_{Z}\left(s_{34}\right)\right) A_{W g}^{(2,4)} \\
& \left.+\left(v_{e}^{\gamma} v_{b}^{\gamma}+v_{e}^{Z} v_{b}^{Z} \mathcal{P}_{Z}\left(s_{34}\right)\right) A_{W g}^{(2,5)}+v_{\nu}^{Z} v_{b}^{Z} \mathcal{P}_{Z}\left(s_{34}\right) A_{W g}^{(2,6)}\right)
\end{aligned}
$$

Here

$$
\begin{gathered}
\mathcal{P}_{Z}(s)=\frac{s}{s-m_{Z}^{2}}, \quad e=g_{w} \sin \theta_{W} \\
v_{f}^{\gamma, L}=-Q, \quad v_{f}^{\gamma, R}=-Q
\end{gathered}
$$




$$
v_{f}^{Z, L}=\frac{I_{3}-Q \sin ^{2} \theta_{W}}{\sin \theta_{W} \cos \theta_{W}}, \quad v_{f}^{Z, R}=\frac{-Q \sin \theta_{W}}{\cos \theta_{W}},
$$

where $Q$ and $I_{3}$ denote the charge and the third component of the weak isospin of the fermion. The labels $L$ and $R$ denote the left- and right-handed couplings. Further, $\theta_{W}$ denotes the Weinberg angle and $V_{u d}$ and $V_{t b}$ denote CKM-matrix elements.

Because the W-boson only couples to left-handed fermions, all non-vanishing amplitudes have the helicity configuration $\left(p_{1}^{-}, p_{2}^{+}, p_{6}^{-}, p_{7}^{+}\right)$. Furthermore the helicity along the fermion line $b\left(p_{3}\right)-\bar{b}\left(p_{4}\right)$ is conserved. Due to their number and length we have collected the explicit expressions for the partial helicity amplitudes $A_{W g}^{(k, l)}$ in $(2.22)$ in Appendix A. We have verified the correctness of these expressions by numerical comparison with the computer code generated by Madgraph [11].

The cross section for W-gluon fusion, summed and averaged over helicities and colors is then given by

$$
\sigma_{W g}=\sum_{i, j} \int d x_{1} f_{i}\left(x_{1}\right) \int d x_{2} f_{j}\left(x_{2}\right) \int d \phi_{5} \frac{1}{8 \hat{s}} \sum_{\text {helicities }} \frac{1}{2}\left(\left|A_{W g}^{(1)}\right|^{2}+\left|A_{W g}^{(2)}\right|^{2}\right) \Theta(\text { cuts })
$$

where $f_{i}\left(x_{1}\right)$ and $f_{j}\left(x_{2}\right)$ are the parton densities of the initial partons $i$ and $j, d \phi_{5}$ denotes the phase space measure for five massless particles, $2 \hat{s}$ is the flux factor, $\Theta$ (cuts) represents the jet-defining cuts and $A_{W g}^{(1)}$ and $A_{W g}^{(2)}$ are the amplitudes in (2.22) with partons $i$ and $j$ crossed into the initial state.

\subsection{Flavor excitation and $s$-channel}

The helicity amplitudes for both these processes can be obtained by crossing from

$$
0 \rightarrow \nu\left(p_{1}\right)+\bar{l}\left(p_{2}\right)+b\left(p_{3}\right)+\bar{b}\left(p_{4}\right)+d\left(p_{6}\right)+\bar{u}\left(p_{7}\right) .
$$

The tree-level amplitudes correspond to order $O\left(g_{w}^{4}\right)$. To this order the color decomposition of the amplitude for (2.25) is simply

$$
A_{W b}=\delta_{34} \delta_{67} A_{W b}^{(1)}
$$

since no gluons are involved. A representative Feynman diagram is shown in Figure 2.3. There are 10 Feynman diagrams contributing to this amplitude, only one of them contains a top quark. We write the partial amplitudes $A_{W b}^{(1)}$ as

$$
A_{W b}^{(1)}=\frac{e^{4} V_{u d}^{*}}{2 \sin ^{2} \theta_{W}}\left(\frac{\left\|V_{t b}\right\|^{2}}{2 \sin ^{2} \theta_{W}} A_{W b}^{(1,1)}+\left(v_{d}^{\gamma} v_{b}^{\gamma}+v_{d}^{Z} v_{b}^{Z} \mathcal{P}_{Z}\left(s_{34}\right)\right) A_{W b}^{(1,2)}\right.
$$




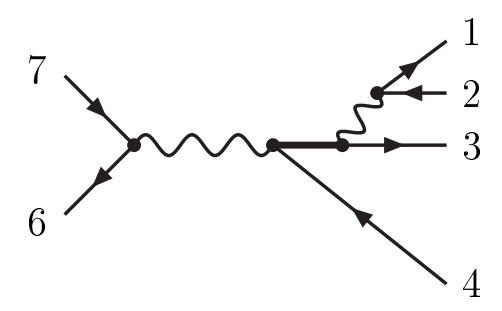

Figure 2.3: A representative Feynman diagram corresponding to the partial amplitude $A_{W b}^{(1)}$ (2.26). The top quark line is thickened.

$$
\begin{aligned}
& +\left(v_{u}^{\gamma} v_{b}^{\gamma}+v_{u}^{Z} v_{b}^{Z} \mathcal{P}_{Z}\left(s_{34}\right)\right) A_{W b}^{(1,3)}+\left(v_{b}^{\gamma}-\frac{\cos \theta_{W}}{\sin \theta_{W}} v_{b}^{Z} \mathcal{P}_{Z}\left(s_{34}\right)\right) A_{W b}^{(1,4)} \\
& \left.+\left(v_{e}^{\gamma} v_{b}^{\gamma}+v_{e}^{Z} v_{b}^{Z} \mathcal{P}_{Z}\left(s_{34}\right)\right) A_{W b}^{(1,5)}+v_{\nu}^{Z} v_{b}^{Z} \mathcal{P}_{Z}\left(s_{34}\right) A_{W b}^{(1,6)}\right)
\end{aligned}
$$

All helicity amplitudes have the helicities $\left(p_{1}^{-}, p_{2}^{+}\right)$and $\left(p_{6}^{-}, p_{7}^{+}\right)$. Again the helicity along the $b-\bar{b}$-line is conserved. The explicit expressions for the $A_{W b}^{(1, k)}$ in $(2.27)$ are collected in Appendix A.

We have checked these results numerically with the computer code produced by Madgraph [11], and found agreement. For the present process we could in addition compare to results produced by the Comphep program [30], and found agreement as well. With the helicity amplitudes at hand one obtains the cross section for flavor excitation, summed and averaged over helicities and colors, as

$$
\sigma_{W b}=\sum_{i, j} \int d x_{1} f_{i}\left(x_{1}\right) \int d x_{2} f_{j}\left(x_{2}\right) \int d \phi_{4} \frac{1}{8 \hat{s}} \sum_{\text {helicities }}\left|A_{W b}^{(1)}\right|^{2} \Theta(\text { cuts })
$$

with $d \phi_{4}$ the phase space measure for 4 massless particles and $i$ and $j$ label the partons crossed for flavor excitation. The cross section for the s-channel process is obtained in a similar way by crossing the appropriate partons.

There are tree diagrams of order $O\left(g^{2} g_{w}^{2}\right)$ that contribute to (2.25). As they are a separately gauge-invariant set and do not contain a top quark they are not directly relevant to us here. However, for this case we do present these "QCD + Weak"amplitudes because they may be easily obtained from the $O\left(g_{w}^{4}\right)$ tree amplitudes, as follows

$$
A_{\mathrm{QCD}+\text { Weak }}=\frac{1}{2}\left(\delta_{37} \delta_{64}-\frac{1}{N_{c}} \delta_{34} \delta_{67}\right) \frac{g^{2} e^{2} V_{u d}^{*}}{2 \sin ^{2} \theta_{W}}\left(A_{W b}^{(1,2)}+A_{W b}^{(1,3)}\right)
$$

where $N_{c}$ denotes the number of colors. In contrast to the process of the previous section, these "QCD + Weak"-amplitudes do not interfere with the $O\left(g_{w}^{4}\right)$ amplitudes. 


\subsection{Narrow width approximation}

By including the top quark semileptonic decay in the amplitudes for (2.6) and (2.7), we must include as well many diagrams in which no top is present. Therefore it is interesting to know to what extent results are approximated by producing the top quark as an on-shell particle, whose decay happens independently from its production.

A numerical indication that the narrow top width approximation for the W-gluon, $\mathrm{s}$-channel and $\mathrm{W}$-associated processes works well is already present in [16]. Here we examine this issue both numerically and analytically for the three subprocesses (2.1), $(2.2)^{3}$ and (2.3). Thus we need the helicity amplitudes for the W-gluon fusion process without top decay

$$
0 \rightarrow t\left(p_{8}\right)+\bar{b}\left(p_{4}\right)+g\left(p_{5}\right)+d\left(p_{6}\right)+\bar{u}\left(p_{7}\right) .
$$

The amplitude may again be color-decomposed as

$$
A_{W g, \text { prod }}=g T_{84}^{a} \delta_{67} A_{W g, \text { prod }}^{(1)}+g \delta_{84} T_{67}^{a} A_{W g, p r o d}^{(2)}
$$

with

$$
\begin{aligned}
A_{W g, p r o d}^{(1)} & =\frac{e^{2} V_{u d}^{*} V_{t b}}{2 \sin ^{2} \theta_{W}} \cdot \frac{(-i) 2 \sqrt{2}}{s_{67}-m_{W}^{2}} \frac{B_{W g, p r o d}^{(1)}}{\sqrt{-\langle 2-|4+5+6+7| 2-\rangle}} \\
A_{W g, p r o d}^{(2)} & =\frac{e^{2} V_{u d}^{*} V_{t b}}{2 \sin ^{2} \theta_{W}} \cdot \frac{(-i) 2 \sqrt{2}}{s_{567}-m_{W}^{2}} \frac{B_{W g, p r o d}^{(2)}}{\sqrt{-\langle 2-|4+5+6+7| 2-\rangle}}
\end{aligned}
$$

As reference momentum for the massive spinor we have chosen $q=p_{2}$. As before all non-vanishing amplitudes have the helicity configuration $\left(p_{6}^{-}, p_{7}^{+}\right)$. The non-vanishing amplitudes are

$$
\begin{aligned}
B_{W g, p r o d}^{(1)}\left(p_{4}^{+}, p_{5}^{+}, p_{8}^{-}\right)= & \frac{\langle 6-|4+5+7| 2-\rangle}{\langle 65\rangle} \\
& \left(\frac{\langle 6-|4+5| 7-\rangle}{\langle 45\rangle}+\frac{[74]\langle 6-|4+7| 5-\rangle}{s_{467}-m^{2}}\right), \\
B_{W g, p r o d}^{(1)}\left(p_{4}^{+}, p_{5}^{+}, p_{8}^{+}\right)= & -\frac{m\langle 26\rangle}{\langle 65\rangle}\left(\frac{\langle 6-|4+5| 7-\rangle}{\langle 45\rangle}+\frac{[74]\langle 6-|4+7| 5-\rangle}{s_{467}-m^{2}}\right), \\
B_{W g, p r o d}^{(1)}\left(p_{4}^{+}, p_{5}^{-}, p_{8}^{-}\right)= & \frac{[74]}{[54]\left(s_{467}-m^{2}\right)}\left(\langle 5-|4+6+7| 2-\rangle[47]\langle 76\rangle+m^{2}[24]\langle 56\rangle\right), \\
B_{W g, p r o d}^{(1)}\left(p_{4}^{+}, p_{5}^{-}, p_{8}^{+}\right)= & -\frac{m}{s_{467}-m^{2}} \frac{[47]}{[45]}(\langle 25\rangle\langle 67\rangle[74]+\langle 56\rangle\langle 2-|5+6+7| 4-\rangle),
\end{aligned}
$$

\footnotetext{
${ }^{3}$ For this channel the narrow width approximation has even been examined to one loop in [8].
} 


$$
\begin{aligned}
B_{W g, \text { prod }}^{(2)}\left(p_{4}^{+}, p_{5}^{+}, p_{8}^{-}\right) & =\frac{\langle 6-|4+5+7| 2-\rangle\langle 6-|5+7| 4-\rangle}{\langle 56\rangle\langle 75\rangle}, \\
B_{W g, \text { prod }}^{(2)}\left(p_{4}^{+}, p_{5}^{+}, p_{8}^{+}\right) & =\frac{m\langle 62\rangle\langle 6-|5+7| 4-\rangle}{\langle 56\rangle\langle 75\rangle}, \\
B_{W g, \text { prod }}^{(2)}\left(p_{4}^{+}, p_{5}^{-}, p_{8}^{-}\right) & =\frac{[74]\langle 2+|(4+5+6+7)(5+6)| 7-\rangle}{[57][56]}, \\
B_{W g, \text { prod }}^{(2)}\left(p_{4}^{+}, p_{5}^{-}, p_{8}^{+}\right) & =\frac{m[47]\langle 2-|5+6| 7-\rangle}{[57][56]} .
\end{aligned}
$$

The helicity amplitudes for flavor excitation and the s-channel process without the top decay

$$
0 \rightarrow t\left(p_{8}\right)+\bar{b}\left(p_{4}\right)+d\left(p_{6}\right)+\bar{u}\left(p_{7}\right)
$$

are relatively simple and are given by

$$
A_{W b, p r o d}=\delta_{84} \delta_{67} \frac{e^{2} V_{u d}^{*} V_{t b}}{2 \sin ^{2} \theta_{W}} \cdot \frac{2 i}{s_{67}-m_{W}^{2}} \frac{B_{W b, p r o d}^{(1)}}{\sqrt{-\langle 2-|4+6+7| 2-\rangle}} .
$$

As reference momentum for the massive spinor we have again chosen $q=p_{2}$. All helicity amplitudes have the configuration $\left(p_{6}^{-}, p_{7}^{+}\right)$. The non-vanishing amplitudes are

$$
\begin{aligned}
& B_{W b, \text { prod }}^{(1)}\left(p_{4}^{+}, p_{8}^{-}\right)=[47]\langle 6-|4+7| 2-\rangle, \\
& B_{W b, \text { prod }}^{(1)}\left(p_{4}^{+}, p_{8}^{+}\right)=m\langle 26\rangle[74] .
\end{aligned}
$$

Finally, let us give the amplitude for the top decay

$$
t\left(p_{8}\right) \rightarrow \nu\left(p_{1}\right)+\bar{l}\left(p_{2}\right)+b\left(p_{3}\right) .
$$

With the choice $q=p_{2}$ as reference momentum for the top-spinor the only nonvanishing amplitude is

$$
A_{d e c}\left(p_{1}^{-}, p_{2}^{+}, p_{3}^{-}, p_{8}^{-}\right)=\frac{e^{2} V_{t b}^{*}}{2 \sin ^{2} \theta_{W}} \frac{2 i}{s_{12}-m_{W}^{2}}\langle 31\rangle \sqrt{\langle 2-|1+3| 2-\rangle}
$$

To implement the narrow top width approximation we keep in the amplitude only terms with a propagator $1 /\left(p_{8}^{2}-m^{2}+i m \Gamma\right)$ with $\Gamma$ the inclusive top width. For the amplitude squared we obtain

$$
\begin{aligned}
|A|^{2} & =\left|\sum_{\lambda} A_{d e c}\left(\ldots, p_{8}^{\lambda}\right) \frac{i}{p_{8}^{2}-m^{2}+i m \Gamma} A_{\text {prod }}\left(\ldots, p_{8}^{\lambda}\right)\right|^{2} \\
& =\left|A_{d e c}\left(\ldots, p_{8}^{-}\right)\right|^{2} \frac{1}{\left(p_{8}^{2}-m^{2}\right)^{2}+m^{2} \Gamma^{2}}\left|A_{\text {prod }}\left(\ldots, p_{8}^{-}\right)\right|^{2}
\end{aligned}
$$


because, with our choice of reference momentum for the massive spinor, $A_{\text {dec }}\left(\ldots, p_{8}^{+}\right)=$ 0 . In the limit of vanishing top width the Breit-Wigner function in (2.40) reduces to a Dirac delta-function and we obtain for the squared amplitude

$$
\frac{\pi}{m \Gamma} \delta\left(p_{8}^{2}-m^{2}\right)\left|A_{d e c}\left(\ldots, p_{8}^{-}\right)\right|^{2}\left|A_{\text {prod }}\left(\ldots, p_{8}^{-}\right)\right|^{2} .
$$

The full $n$-particle phase space may be factorized accordingly

$$
d \phi_{n}\left(Q \rightarrow k_{1}, \ldots, k_{n}\right)=\frac{1}{2 \pi} d \phi_{n-2}\left(Q \rightarrow p_{8}, k_{4}, \ldots, k_{n}\right) d p_{8}^{2} d \phi_{3}\left(p_{8} \rightarrow k_{1}, k_{2}, k_{3}\right),
$$

with $n=5$ for the $\mathrm{W}$-gluon fusion and $\mathrm{W}$-associated production, and $n=4$ for flavor excitation and $s$-channel production. Note that

$$
\int\left|A_{d e c}\left(\ldots, p_{8}^{-}\right)\right|^{2} d \phi_{3}\left(p_{8} \rightarrow k_{1}, k_{2}, k_{3}\right)=2 m \Gamma_{\nu \overline{l b}} .
$$

Numerical results for the narrow top-width approximation are presented in the next section.

\subsection{Numerical studies}

As announced in the introduction, we consider each subprocess separately for the purposes of numerical studies. This is equivalent to assuming a hermetic detector with perfect momentum resolution capable of distinguishing these three processes.

Before describing our numerical studies we list our default choices for physical constants and parameters. For the masses and widths of the electroweak bosons we use $m_{Z}=91.187 \mathrm{GeV}, \Gamma_{Z}=2.49 \mathrm{GeV}, m_{W}=80.41 \mathrm{GeV}$ and $\Gamma_{W}=2.06 \mathrm{GeV}$. For the top quark mass we use $m_{t}=174 \mathrm{GeV}$. The width of the top quark is then calculated as $\Gamma_{t}=1.76 \mathrm{GeV}$. We use the leading order expression for the running of the strong coupling constant:

$$
\alpha_{s}(\mu)=\alpha_{s}\left(m_{Z}\right)\left[1+\frac{\alpha_{s}\left(m_{Z}\right)}{4 \pi} \beta_{0} \ln \frac{\mu^{2}}{m_{Z}^{2}}\right]^{-1},
$$

where $\beta_{0}=11-\frac{2}{3} N_{f}$ and $N_{f}=5$. We use the CTEQ4L set for the parton densities [31] and we take therefore $\alpha_{s}\left(m_{Z}\right)=0.132$. The running of the finestructure constant is taken in account according to

$$
\alpha(\mu)=\alpha(0)\left[1-\Delta \alpha\left(m_{Z}\right)-\frac{\alpha(0)}{3 \pi}\left(\frac{20}{3} \ln \frac{\mu^{2}}{m_{Z}^{2}}-\frac{4}{15} \frac{\left(\mu^{2}-m_{Z}^{2}\right)}{m_{t}^{2}}\right)\right]^{-1},
$$

with $\alpha(0)=1 / 137.036$ and $\Delta \alpha\left(m_{Z}\right)=0.059363([32,33,34,35])$. We consider $p-\bar{p}$ collisions at a center of mass energy $\sqrt{S}=2.0 \mathrm{TeV}$ (Tevatron) and $p$ - $p$ collisions at a 
center of mass energy $\sqrt{S}=14 \mathrm{TeV}$ (LHC). For the renormalization and factorization scale we use $\mu=\mu_{F}=m_{t}$.

Although defining jets is not strictly necessary for a LO calculation, we use them to avoid singularities. By requiring as many jets as there are partons in the event, collinear and soft divergent regions are avoided. Jets are defined by the hadronic $k_{T^{-}}$ algorithm [36]: we first remove the charged lepton and the neutrino from the event, then we precluster all remaining particles and assign them to the beamjets or to the hard scattering process. Particles which are assigned to the hard scattering process are then clustered into jets. For the resolution variable of the hadronic $k_{T}$-algorithm we use

$$
y_{i j}=2 \min \left(p_{T i}^{2}, p_{T j}^{2}\right)\left(\cosh \left(y_{i}-y_{j}\right)-\cos \left(\phi_{i}-\phi_{j}\right)\right),
$$

where $p_{T i}$ is the transverse momentum, $y_{i}$ the rapidity and $\phi_{i}$ the polar angle of particle $i$. We recombine two particles using the $E$-scheme. For the preclustering we use $d_{\text {cut }}=(20 \mathrm{GeV})^{2}$. The clustering is done with $y_{\text {cut }}=0.9$. We have implemented the finite width of the $W, Z$-bosons and of the top quark by using the complex-mass scheme [37] which respects full gauge invariance and which therefore gives a consistent description of the finite-width effects in tree-level calculations. Thus, the masses $m_{W}$, $m_{Z}$ and $m_{t}$ in the partial amplitudes are replaced according to

$$
m \rightarrow \sqrt{m^{2}-i \Gamma m}
$$

As a consequence the cosine squared of the Weinberg angle also becomes a complex number

$$
\cos ^{2} \theta_{W}=1-\sin ^{2} \theta_{W}=\frac{m_{W}^{2}-i \Gamma_{W} m_{W}}{m_{Z}^{2}-i \Gamma_{Z} m_{Z}}
$$

We give our results for single-top production only. Furthermore we concentrate most of our studies on the Tevatron. LHC kinematics reweights the various processes among each other, the amount of which is not so much our concern in this thesis (see [16] e.g.).

Although we do not combine the partonic subprocesses, and rather examine them individually, we wish to define their final state in a semi-realistic manner. Specifically, we keep the partons apart in phase space by means of a jet algorithm (this includes in particular, for the W-gluon channel, the beam and the $\bar{b}$ jet). However, we do assume perfect $b$-tagging, and no mistagging.

The inclusion of single-antitop production will multiply the cross section by a factor of two at the Tevatron. This is not the case at the LHC, which is a proton-proton collider. The numerical results are for one light lepton species only, e.g. $\bar{l}=e^{+}$and $\nu=\nu_{e}$. The inclusion of the muon-channel multiplies every result by two. 


\begin{tabular}{c|c|c|c}
\hline \hline Tevatron & $\sigma_{\text {tot }}$ & $\left|m_{\nu \overline{l b}}-m_{t}\right|<20 \mathrm{GeV}$ & narrow width \\
\hline \hline W-gluon fusion & $15.0 \pm 0.4 \mathrm{fb}$ & $14.3 \pm 0.3 \mathrm{fb}$ & $14.5 \pm 0.1 \mathrm{fb}$ \\
\hline flavor excitation & $87 \pm 1 \mathrm{fb}$ & $85 \pm 2 \mathrm{fb}$ & $87 \pm 1 \mathrm{fb}$ \\
\hline$s$-channel & $46 \pm 1 \mathrm{fb}$ & $32.3 \pm 0.3 \mathrm{fb}$ & $29.0 \pm 0.2 \mathrm{fb}$ \\
\hline \hline
\end{tabular}

Table 2.1: Numerical results for the Tevatron at $2 \mathrm{TeV}$.

\begin{tabular}{c|c|c|c}
\hline \hline LHC & $\sigma_{\text {tot }}$ & $\left|m_{\nu \overline{l b}}-m_{t}\right|<20 \mathrm{GeV}$ & narrow width \\
\hline \hline W-gluon fusion & $4.6 \pm 0.2 \mathrm{pb}$ & $4.5 \pm 0.4 \mathrm{pb}$ & $4.6 \pm 0.1 \mathrm{pb}$ \\
\hline flavor excitation & $13.1 \pm 0.3 \mathrm{pb}$ & $13.0 \pm 0.4 \mathrm{pb}$ & $13.3 \pm 0.1 \mathrm{pb}$ \\
\hline s-channel & $685 \pm 19 \mathrm{fb}$ & $479 \pm 16 \mathrm{fb}$ & $432 \pm 4 \mathrm{fb}$ \\
\hline \hline
\end{tabular}

Table 2.2: Numerical results for the LHC at $14 \mathrm{TeV}$.

For $\mathrm{W}$-gluon fusion we require three jets, two of them $b$-tagged, for flavor excitation two jets with one $b$-tag, whereas for the $s$-channel process we require two $b$-tagged jets. For simplicity we assume a $b$-tagging efficiency of $100 \%$, and that we know the longitudinal momentum component of the neutrino ${ }^{4}$.

In Table 2.1 we give the numerical results for the total cross section with the cuts described above for W-gluon fusion, flavor excitation and $s$-channel process at the Tevatron (first column). In the second column we required in addition that the invariant mass of the decay products of the top reconstruct to within $20 \mathrm{GeV}$ to the top quark mass. The third column contains the results in the narrow width approximation. Table 2.2 shows the corresponding results for the LHC. From Table 2.1 and Table 2.2 we see that the narrow width approximation describes the cross section very well for W-gluon fusion and flavor excitation. The approximation is less satisfactory for the s-channel process. Here non-resonant terms seem to give a more sizeable contribution. This can also be seen in Figure 2.4(a), which shows the distribution in the invariant mass $m_{\nu \bar{l} b}$ for $\mathrm{W}$-gluon fusion and the s-channel process at the Tevatron.

In Figure 2.4(b) we show for the $\mathrm{W}$-gluon fusion process at the Tevatron the distribution of the pseudorapidities for the $\bar{b}$-quark, the $b$-quark and the light quark $q$. The distribution for the $\bar{b}$-quark is slightly peaked in the backward region, the $b$-quark is almost central and the light quark goes dominantly in the forward region. Note that the jet algorithm suppresses $\bar{b}$ 's at sizeable negative pseudo-rapidities. These distributions essentially agree with Figure 7 in [39] and Figure 8 in [15].

In W-gluon fusion or flavor excitation the produced top quark is highly polarized along the direction of the $\bar{d}$-quark $[40,41]$. Furthermore the cross section at the Teva-

\footnotetext{
${ }^{4}$ E.g. from imposing the $W$ mass constraint on the neutrino plus lepton invariant mass [38].
} 


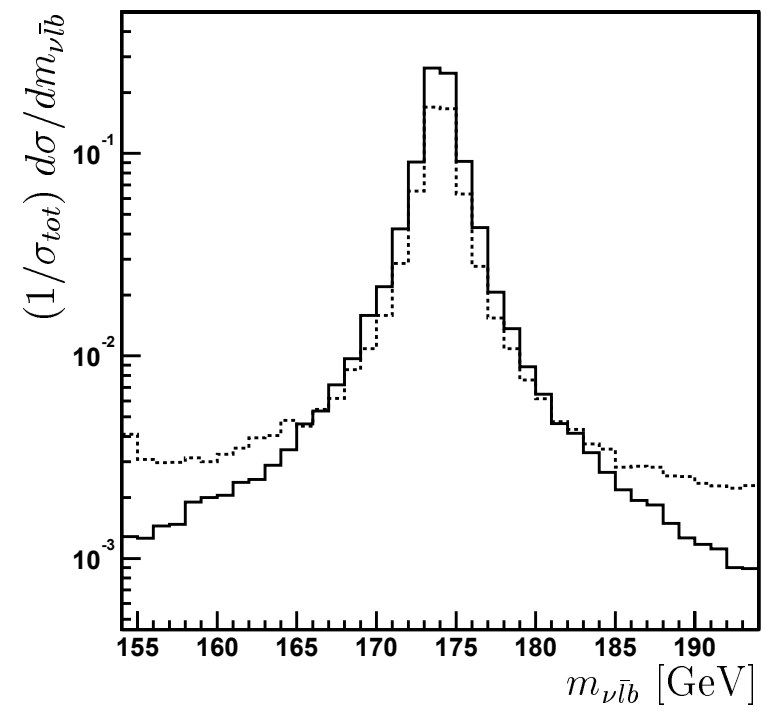

(a)

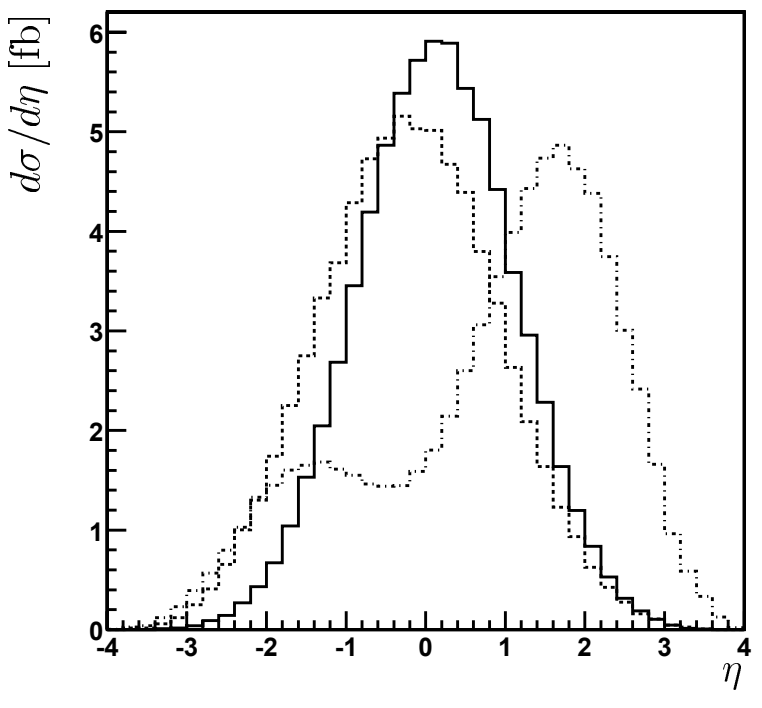

(b)

Figure 2.4: (a) The normalized $m_{\nu \bar{l} b}$ distribution for $W$-gluon fusion (solid) and the s-channel process (dashed) at the Tevatron. (b) The pseudo-rapidity distribution for $W$-gluon fusion of the $\bar{b}$-quark (dashed), the b-quark (solid) and the light quark $q$ (dot-dashed) at the Tevatron.
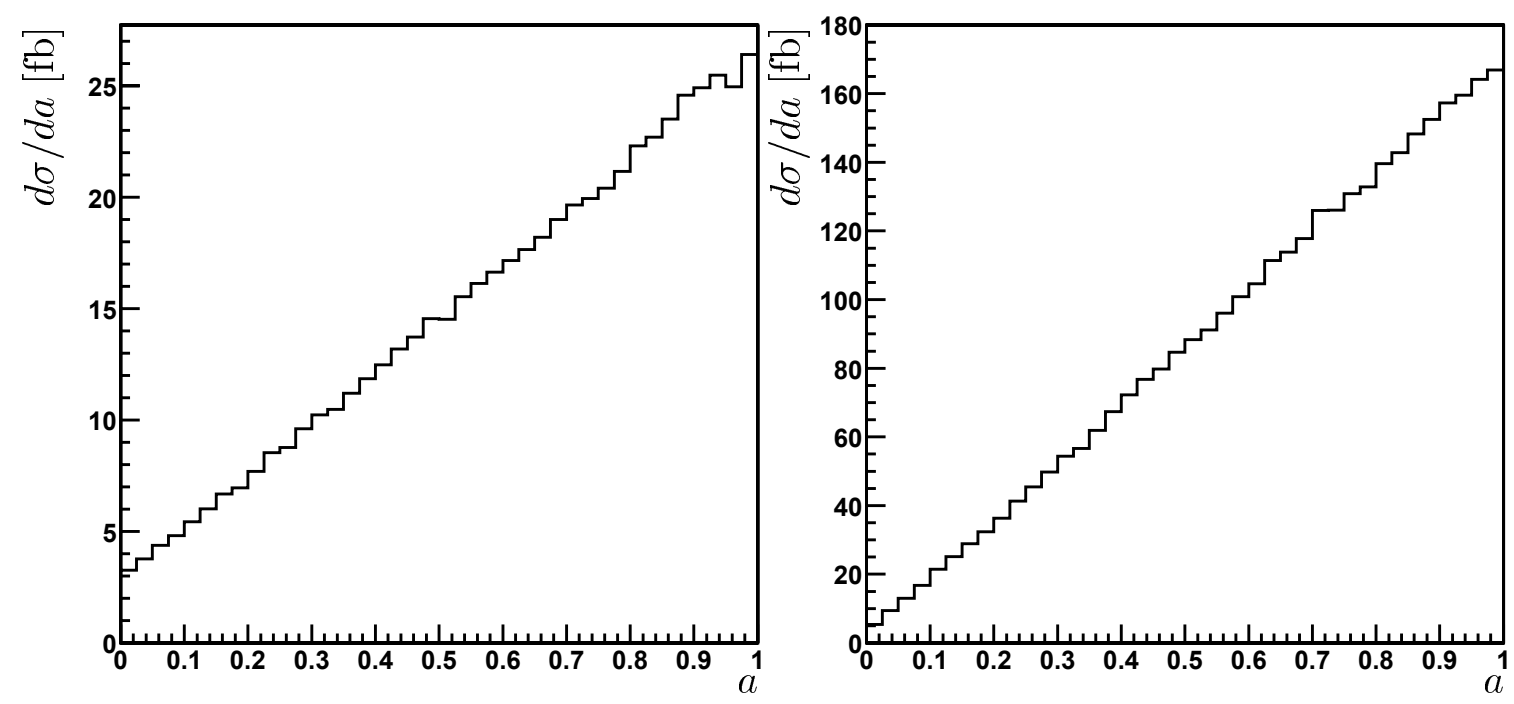

Figure 2.5: The distribution for the angular correlation a for $W$-gluon fusion (left) and flavor excitation (right) at the Tevatron. 


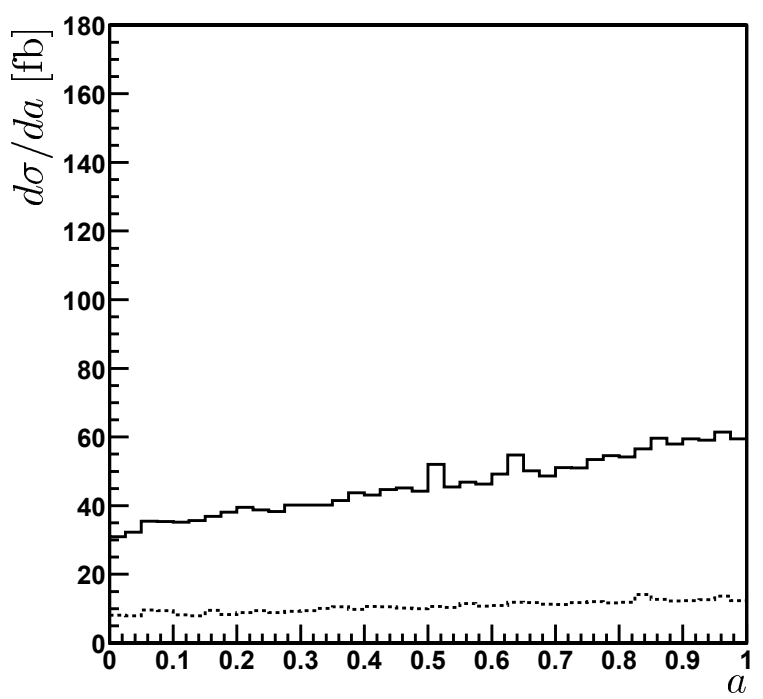

Figure 2.6: The distribution for the angular correlation a for the "QCD + Weak" background to flavor excitation at the Tevatron. We show the distribution with (dashed) and without (solid) a cut on the invariant mass $\left|m_{\nu \overline{l b}}-m_{t}\right|<20 \mathrm{GeV}$.

tron receives the dominant contribution from the configuration where the $u$-quark is in the initial state and the $\bar{d}$-quark in the final state, which in turn produces the non- $b$ tagged jet $q$. One considers therefore the variable

$$
a=\frac{1}{2}\left(1+\cos \theta_{q \bar{l}}\right)
$$

where $\theta_{q \bar{l}}$ is the angle between the non- $b$ tagged jet and the charged lepton in the rest frame of $p_{\nu}+p_{\bar{l}}+p_{b}$ [42]. (If a top is produced, $p_{\nu}+p_{\bar{l}}+p_{b}$ corresponds to its four-momentum.) For the angular correlation of a decaying top quark one has [43]

$$
\frac{d \sigma}{d a}=\sigma(2 P a+(1-P))
$$

where $P$ is the polarization of the top quark along the spin axis defined by the spectator jet $q$. For a $100 \%$ polarized top quark one has therefore $d \sigma / d a \sim a$. Figure 2.5 shows that this relation is fulfilled to a very good approximation for flavor excitation. For $\mathrm{W}$-gluon fusion we obtain the polarization along the spectator jet axis from the value at $a=0$ :

$$
P=1-\left.\frac{1}{\sigma} \frac{d \sigma}{d a}\right|_{a=0} .
$$

With the total cross section from Table 2.1 we find $P=77 \%$ for W-gluon fusion. The background is expected to give a flat distribution. For flavor excitation we show 
the result for the "QCD + Weak" background (discussed below (2.28)) process in Figure 2.6. The $a$ dependence after imposing a cut on the invariant mass $m_{\nu \bar{l} b}$ is flat, as expected. Our result is similar to that shown in Figure 5 of [16], which shows the same linear correlation of the signal (which is somewhat different from ours by employing a vetoed $\bar{b}$ jet), and shows the (flat) $a$ dependence of their more extensively treated background as well.

We suggest that this clear correlation may provide an alternative and attractive way to infer the visible $\mathrm{W}$-gluon fusion or flavor excitation cross section (defined here through the criteria given above) for single-top production from the slope of the distribution. The slope is given by

$$
2 P_{\text {signal }} \sigma_{\text {signal }}+2 P_{\text {background }} \sigma_{\text {background }} .
$$

Assuming that $P_{\text {background }} \sigma_{\text {background }}$ is small and that $P_{\text {signal }}$ may reliable be estimated from theory the visible cross section for the signal can be inferred from the slope by measuring two or more points of the $a$ distribution and extrapolating the distribution to a straight line. Although in principle of course any distribution may serve to infer the corresponding inclusive cross section, the $a$ distribution seems particularly attractive due to its simple shape.

\subsection{Conclusions}

We have presented the complete $O\left(g_{w}^{4}\right)$ and $O\left(g g_{w}^{4}\right)$ helicity amplitudes for processes whose final state result from the hadroproduction and semileptonic decay of a single top.

As only a small subset of graphs actually contain a top quark line in each process, we have examined, for three of these processes, to what extent the top quark dominates, and verified that for each process the top quark presence is manifested by a clear peak in the $m_{\nu \bar{l} b}$ distribution. We have studied various kinematic distribution of final state particles, and verified the correlation of the lepton angular distribution with the top quark polarization $[40,16,41]$. The actual identification of a single-top signal is a matter of careful definition, requiring full use of the kinematic and flavor characteristics of the final state, and a proper determination of the acceptance and background [16, $18,44]$. In idealized analyses, we have thus verified that the sensitivity of the full amplitudes to top quark mass, charged-current coupling strength and handedness are preserved in these amplitudes, even though most diagrams that contribute to them do not contain a top quark.

Now that we have completed the leading order calculation, the next step is to tackle the next to leading order calculation. For this, we would like to use the most advanced 
method available, the dipole method [45]. We will describe this method and develop its extension to massive particles in the next chapter. 


\section{Chapter 3}

\section{Massive Dipole Formalism}

\subsection{Introduction}

Reliable theoretical predictions of hard-scattering processes in QCD require at least the evaluation of Next to Leading Order (NLO) QCD corrections. Next to leading order calculations have to combine virtual one-loop corrections with the real emission contributions from unresolved partons. Treated separately, each of the two parts gives an infrared divergent contribution. Only the sum of the virtual corrections and the real emission contributions is infrared finite. Setting up a numerical general-purpose NLO Monte Carlo program requires therefore the analytical cancellation of infrared singularities before any numerical integration can be done. The two main methods to handle this task are phase space slicing $[46,47,48]$ and the subtraction method $[49,50]$. For massless partons both methods are available and have been applied to a variety of specific processes, see for example Refs. $[51,52,53,54,55]$ and the references therein. For electron-positron annihilation a general formulation of phase space slicing has been given by Giele and Glover [56]. It has been extended to initial-state partons by these authors and Kosower [57]. The extension of phase space slicing to massive partons and identified hadrons has been given by Laenen and Keller [58].

There are two general formulations of the subtraction method. One is the residue approach by Frixione, Kunzst and Signer [59] and the other the dipole formalism by Catani and Seymour [45]. Both variants can handle massless partons in the final and/or initial state. The subtraction method has already been applied to some specific processes with massive partons $[60,61,62,63]$. Up to now there is no extension of the dipole formalism to massive partons. Dittmaier has considered the dipole formalism for photon radiation from fermions [64]. In that work infrared divergences are regularized with small masses (as it is popular in electroweak physics). However this does not allow a simple application to QCD, where divergences are usually regularized by dimensional regularization. 
In this chapter we extend the dipole formalism to heavy fermions. The formulae we provide are relevant to top, bottom and charm production. With a simple change of the color factors they can be used as well for gluino production in SUSY QCD. Our results are not applicable for processes with identified hadrons in the final state, massive initial-state partons and processes with different species of massive fermions of unequal masses.

One-loop amplitudes may be calculated in different variants of dimensional regularization, such as conventional dimensional regularization (CDR), the 't Hooft-Veltman scheme (HV) or the four-dimensional scheme (FD), whereas the Born amplitudes entering the real emission part and/or parton densities are given in another variant. Of course, the final result has to be scheme-independent. The obvious way to ensure this is to calculate everything in the same scheme. Often this is not the most economical solution. Loop amplitudes are most easily computed in the FD scheme, whereas parton densities are given in the conventional scheme. Within the dipole formalism it is possible to perform different parts of the calculation with different variants of dimensional regularization and to correct for the mismatch by some universal terms [65]. We would like to keep this freedom also in the extension to massive partons. We therefore keep track of all scheme-dependent terms and our final results can be used with any variant of dimensional regularization (CDR, HV or FD).

Recently, Catani, Dittmaier and Trocsanyi considered the singular behavior of oneloop QCD and supersymmetric QCD amplitudes with massive partons in the dipole framework [66]. As a byproduct, we confirm their results for the singular terms.

This chapter is organized as follows. In the next section we review the dipole formalism and the factorization of QCD amplitudes in the soft and collinear limit. In Section 3.3 we outline our calculational technique. In Section 3.4 we derive the $D$ dimensional dipole phase space measure. Section 3.5 gives the dipole terms together with the integrated counterpart if all particles are in the final state. In Section 3.6 we consider the case if there are QCD partons in the initial state. Finally, Section 3.7 contains our conclusions.

\subsection{Dipole formalism and factorization in singular limits}

In this section we briefly review the dipole formalism and the factorization properties of QCD amplitudes in the soft and collinear limit. We use the notation of Catani and Seymour [45].

The dipole formalism is based on the subtraction method. We explain it for electronpositron annihilation, where all QCD partons are in the final state. The NLO cross 
section is rewritten as

$$
\begin{aligned}
\sigma^{N L O} & =\int_{n+1} d \sigma^{R}+\int_{n} d \sigma^{V} \\
& =\int_{n+1}\left(d \sigma^{R}-d \sigma^{A}\right)+\int_{n}\left(d \sigma^{V}+\int_{1} d \sigma^{A}\right) .
\end{aligned}
$$

In the second line an approximation term $d \sigma^{A}$ has been added and subtracted. The approximation $d \sigma^{A}$ has to fulfill the following requirements:

- $d \sigma^{A}$ must be a proper approximation of $d \sigma^{R}$ such as to have the same pointwise singular behavior (in $D$ dimensions) as $d \sigma^{R}$ itself. Thus, $d \sigma^{A}$ acts as a local counterterm for $d \sigma^{R}$ and one can safely perform the limit $\varepsilon \rightarrow 0$. This defines a cross section contribution

$$
\sigma_{\{n+1\}}^{N L O}=\int_{n+1}\left(\left.d \sigma^{R}\right|_{\varepsilon=0}-\left.d \sigma^{A}\right|_{\varepsilon=0}\right) .
$$

- Analytic integrability (in $D$ dimensions) over the one-parton subspace leading to soft and collinear divergences. This gives the contribution

$$
\sigma_{\{n\}}^{N L O}=\int_{n}\left(d \sigma^{V}+\int_{1} d \sigma^{A}\right)_{\varepsilon=0} .
$$

The final structure of an NLO calculation is then

$$
\sigma^{N L O}=\sigma_{\{n+1\}}^{N L O}+\sigma_{\{n\}}^{N L O} .
$$

Since both contributions on the r.h.s of eq.(3.4) are now finite, they can be evaluated with numerical methods. The $(n+1)$ matrix element is approximated by a sum of dipole terms

$$
\begin{aligned}
& \sum_{\text {pairs }} \sum_{i, j} \mathcal{D}_{i j, k}= \\
& =\sum_{\text {pairs } i, j, j} \sum_{k \neq i, j}-\frac{1}{2 p_{i} \cdot p_{j}}\left\langle 1, \ldots,(\tilde{i j}), \ldots, \tilde{k}, \ldots\left|\frac{\mathbf{T}_{k} \cdot \mathbf{T}_{i j}}{\mathbf{T}_{i j}^{2}} V_{i j, k}\right| 1, \ldots,(\tilde{i j}), \ldots, \tilde{k}, \ldots\right\rangle,
\end{aligned}
$$

where the emitter parton is denoted by $\tilde{i j}$ and the spectator by $\tilde{k}$. Here $\mathbf{T}_{i}$ denotes the color charge operator [45] for parton $i$ and $V_{i j, k}$ is a matrix in the helicity space of the emitter with the correct soft and collinear behavior. $|1, \ldots,(\tilde{i j}), \ldots, \tilde{k}, \ldots\rangle$ is a 
vector in color- and helicity space. By subtracting from the real emission part the fake contribution we obtain

$$
\begin{aligned}
d \sigma^{R}-d \sigma^{A}= & d \phi_{n+1}\left[\left|M\left(p_{1}, \ldots, p_{n+1}\right)\right|^{2} \theta_{n+1}^{\text {cut }}\left(p_{1}, \ldots, p_{n+1}\right)\right. \\
& \left.-\sum_{\text {pairs } i, j} \sum_{k \neq i, j} \mathcal{D}_{i j, k}\left(p_{1}, \ldots, p_{n+1}\right) \theta_{n}^{\text {cut }}\left(p_{1}, \ldots, \tilde{p}_{i j}, \ldots, \tilde{p}_{k}, \ldots, p_{n+1}\right)\right] .
\end{aligned}
$$

Both $d \sigma^{R}$ and $d \sigma^{A}$ are integrated over the same $(n+1)$ parton phase space, but it should be noted that $d \sigma^{R}$ is proportional to $\theta_{n+1}^{c u t}$, whereas $d \sigma^{A}$ is proportional to $\theta_{n}^{c u t}$. Here $\theta_{n}^{\text {cut }}$ denotes the jet-defining function for $n$-partons.

The subtraction term can be integrated over the one-parton phase space to yield the term

$$
\mathbf{I} \otimes d \sigma^{B}=\int_{1} d \sigma^{A}=\sum_{\text {pairs } i, j} \sum_{k \neq i, j} \int d \phi_{\text {dipole }} \mathcal{D}_{i j, k} .
$$

The universal factor I still contains color correlations, but does not depend on the unresolved parton $j$. The term $\mathbf{I} \otimes d \sigma^{B}$ lives on the phase space of the $n$-parton configuration and has the appropriate singularity structure to cancel the infrared divergences coming from the one-loop amplitude. Therefore

$$
d \sigma^{V}+\mathbf{I} \otimes d \sigma^{B}
$$

is infrared finite and can easily be integrated by Monte Carlo methods. The explicit forms of the dipole terms $\mathcal{D}_{i j, k}$, together with the integrated counterparts, can be found in Ref. [45] for the massless case.

In order to extend the dipole formalism to massive fermions, we have to provide three ingredients. First, we need the correct subtraction terms for the real emission contribution. Second, we have to integrate these subtraction terms over the dipole phase space. The integrated terms are combined with the virtual corrections. Third, we have to specify a mapping of the momenta, which relates the $(n+1)$-parton configuration $p_{1}, \ldots, p_{i}, \ldots, p_{j}, \ldots p_{k}, \ldots p_{n+1}$ to the $n$-parton configuration $p_{1}, \ldots, \tilde{p}_{i j}, \ldots, \tilde{p}_{k}, \ldots, p_{n+1}$.

In order to find appropriate dipole terms, one considers the soft and collinear limits of the matrix element. In the soft limit where the momentum of parton $j$ becomes soft, the $m+1$-parton matrix element behaves as

$$
\langle 1, \ldots, m+1 \mid 1, \ldots, m+1\rangle=-4 \pi \mu^{2 \varepsilon} \alpha_{s}\left\langle 1, \ldots, m\left|\mathbf{J}^{\mu \dagger} \mathbf{J}_{\mu}\right| 1, \ldots, m\right\rangle
$$

with

$$
\mathbf{J}^{\mu \dagger} \mathbf{J}_{\mu}|1, \ldots, m\rangle=\left(2 \sum_{i \neq k} \frac{2 p_{i} p_{k}}{\left(2 p_{i} p_{j}\right)\left(2 p_{j} p_{k}\right)} \mathbf{T}_{i} \cdot \mathbf{T}_{k}+\sum_{i} \frac{4 m_{i}^{2}}{\left(2 p_{i} p_{j}\right)^{2}} \mathbf{T}_{i} \cdot \mathbf{T}_{i}\right)|1, \ldots, m\rangle
$$




$$
=4 \sum_{i \neq k}\left(\frac{2 p_{i} p_{k}}{\left(2 p_{i} p_{j}\right)\left(2 p_{i} p_{j}+2 p_{j} p_{k}\right)}-\frac{m_{i}^{2}}{\left(2 p_{i} p_{j}\right)^{2}}\right) \mathbf{T}_{i} \cdot \mathbf{T}_{k}|1, \ldots, m\rangle
$$

We used

$$
\frac{2 p_{i} p_{k}}{\left(2 p_{i} p_{j}\right)\left(2 p_{j} p_{k}\right)}=\frac{2 p_{i} p_{k}}{\left(2 p_{i} p_{j}\right)\left(2 p_{i} p_{j}+2 p_{j} p_{k}\right)}+\frac{2 p_{i} p_{k}}{\left(2 p_{j} p_{k}\right)\left(2 p_{i} p_{j}+2 p_{j} p_{k}\right)}
$$

and color conservation

$$
\sum_{i} \mathbf{T}_{i}|1, \ldots, m\rangle=0
$$

The color charge operators $\mathbf{T}_{i}$ for a quark, gluon and antiquark in the final state are

$$
\begin{aligned}
\text { quark : } & \left\langle\ldots q_{i} \ldots\left|T_{i j}^{a}\right| \ldots q_{j} \ldots\right\rangle, \\
\text { gluon : } & \left\langle\ldots g^{c} \ldots\left|i f^{c a b}\right| \ldots g^{b} \ldots\right\rangle, \\
\text { antiquark : } & \left\langle\ldots \bar{q}_{i} \ldots\left|\left(-T_{j i}^{a}\right)\right| \ldots \bar{q}_{j} \ldots\right\rangle .
\end{aligned}
$$

If the particles are massless, there is also a singularity in the collinear limit. For final-state partons the momenta are parameterized as

$$
\begin{aligned}
p_{i} & =\hat{z} p+k_{\perp}-\frac{k_{\perp}^{2}}{\hat{z}} \frac{n}{2 p n}, \\
p_{j} & =(1-\hat{z}) p-k_{\perp}-\frac{k_{\perp}^{2}}{1-\hat{z}} \frac{n}{2 p n} .
\end{aligned}
$$

Here $n$ is a massless four-vector and the transverse component $k_{\perp}$ satisfies $2 p k_{\perp}=$ $2 n k_{\perp}=0$. The collinear limit occurs for $k_{\perp}^{2} \rightarrow 0$. In this limit the matrix element behaves as

$$
\langle 1, \ldots, m+1 \mid 1, \ldots, m+1\rangle=4 \pi \mu^{2 \varepsilon} \alpha_{s}\left\langle 1, \ldots, m\left|\frac{2}{2 p_{i} p_{j}} \hat{P}_{(i j), i}\left(\hat{z}, k_{\perp}, \varepsilon\right)\right| 1, \ldots, m\right\rangle .
$$

The splitting functions are given by

$$
\begin{aligned}
\left\langle s\left|\hat{P}_{q q}\left(z, k_{\perp}, \varepsilon\right)\right| s^{\prime}\right\rangle & =\delta_{s s^{\prime}} C_{F}\left[\frac{2 z}{1-z}+(1-\rho \varepsilon)(1-z)\right] \\
\left\langle s\left|\hat{P}_{q g}\left(z, k_{\perp}, \varepsilon\right)\right| s^{\prime}\right\rangle & =\delta_{s s^{\prime}} C_{F}\left[\frac{2(1-z)}{z}+(1-\rho \varepsilon) z\right] \\
\left\langle\mu\left|\hat{P}_{g q}\left(z, k_{\perp}, \varepsilon\right)\right| \nu\right\rangle & =T_{R}\left[-g^{\mu \nu}+4 z(1-z) \frac{k_{\perp}^{\mu} k_{\perp}^{\nu}}{k_{\perp}^{2}}\right] \\
\left\langle\mu\left|\hat{P}_{g g}\left(z, k_{\perp}, \varepsilon\right)\right| \nu\right\rangle & =2 C_{A}\left[-g^{\mu \nu}\left(\frac{z}{1-z}+\frac{1-z}{z}\right)-2(1-\rho \varepsilon) z(1-z) \frac{k_{\perp}^{\mu} k_{\perp}^{\nu}}{k_{\perp}^{2}}\right] .
\end{aligned}
$$


We introduced the parameter $\rho$, which specifies the variant of dimensional regularization: $\rho=1$ for the CDR/HV schemes and $\rho=0$ for the FD scheme. Later on we will chose the dipole terms to have the same soft and collinear behavior as the appropriate limit of the $(n+1)$-parton matrix element.

If the emitting particle is in the initial state the collinear limit is defined as

$$
\begin{aligned}
p_{a} & =p \\
p_{i} & =(1-x) p+k_{\perp}-\frac{k_{\perp}^{2}}{1-x} \frac{n}{2 p n}, \\
p_{a i} & =x p-k_{\perp}+\frac{k_{\perp}^{2}}{1-x} \frac{n}{2 p n} .
\end{aligned}
$$

The color charge operators for a quark, gluon and antiquark in the initial state are

$$
\begin{aligned}
\text { quark : } & \left\langle\ldots \bar{q}_{i} \ldots\left|\left(-T_{j i}^{a}\right)\right| \ldots \bar{q}_{j} \ldots\right\rangle, \\
\text { gluon : } & \left\langle\ldots g^{c} \ldots\left|i f^{c a b}\right| \ldots g^{b} \ldots\right\rangle, \\
\text { antiquark : } & \left\langle\ldots q_{i} \ldots\left|T_{i j}^{a}\right| \ldots q_{j} \ldots\right\rangle .
\end{aligned}
$$

We denoted in the amplitude an incoming quark as an outgoing antiquark and vice versa.

\subsection{Calculational technique for the integration}

The integration of the dipole terms over the dipole phase space is highly non-trivial. We first find a suitable parameterization of the phase space such that all integrals are of one of the following types:

$$
\begin{aligned}
\int_{0}^{1} d x x^{a-1}(1-x)^{c-a-1} & =\frac{\Gamma(a) \Gamma(c-a)}{\Gamma(c)}, \\
\int_{0}^{1} d x x^{a-1}(1-x)^{c-a-1}\left(1-x_{0} x\right)^{-b} & =\frac{\Gamma(a) \Gamma(c-a)}{\Gamma(c)}{ }_{2} F_{1}\left(a, b ; c, x_{0}\right), \\
\int_{0}^{1} d x x^{a-1}(1-x)^{c-a-1}\left(1-x_{1} x\right)^{-b_{1}}\left(1-x_{2} x\right)^{-b_{2}} & =\frac{\Gamma(a) \Gamma(c-a)}{\Gamma(c)} F_{1}\left(a, b_{1}, b_{2} ; c ; x_{1}, x_{2}\right) .
\end{aligned}
$$

These integrals yield the Euler-Beta function, the hypergeometric function and the first Appell function, respectively. The last two are then rewritten as a Taylor series in $x_{0}$ 
or $x_{1}$ and $x_{2}$, respectively.

$$
\begin{aligned}
{ }_{2} F_{1}\left(a, b ; c, x_{0}\right) & =\sum_{n=0}^{\infty} \frac{(a)_{n}(b)_{n}}{(c)_{n}} \frac{x_{0}^{n}}{n !}, \\
F_{1}\left(a, b_{1}, b_{2} ; c ; x_{1}, x_{2}\right) & =\sum_{m_{1}=0}^{\infty} \sum_{m_{2}=0}^{\infty} \frac{(a)_{m_{1}+m_{2}}\left(b_{1}\right)_{m_{1}}\left(b_{2}\right)_{m_{2}}}{(c)_{m_{1}+m_{2}}} \frac{x_{1}^{m_{1}}}{m_{1} !} \frac{x_{2}^{m_{2}}}{m_{2} !} .
\end{aligned}
$$

Here $(a)_{n}=\Gamma(a+n) / \Gamma(a)$ denotes the Pochhammer symbol. Taylor expansion allows us to perform the next integral, which is of the same type as above. After all integrals have been performed, we end up with multiple sums involving $\Gamma$-functions depending on $\varepsilon=(4-D) / 2$. These $\Gamma$-functions are then expanded according to

$$
\begin{aligned}
& \frac{\Gamma(n+\varepsilon)}{\Gamma(n) \Gamma(\varepsilon)}= \\
& \quad=\varepsilon\left(1+\varepsilon Z_{1}(n-1)+\varepsilon^{2} Z_{11}(n-1)+\varepsilon^{3} Z_{111}(n-1)+\ldots+\varepsilon^{n-1} Z_{11 \ldots 1}(n-1)\right)
\end{aligned}
$$

where $Z_{11 \ldots 1}(n)$ are Euler-Zagier sums defined by

$$
Z_{m_{1}, \ldots, m_{k}}(n)=\sum_{i_{1}>i_{2}>\ldots>i_{k}>0}^{n} \frac{1}{i_{1}^{m_{1}}} \frac{1}{i_{2}^{m_{2}}} \ldots \frac{1}{i_{k}^{m_{k}}} .
$$

Rearranging summation indices we recognize that all sums fall in the class of Goncharov's multiple polylogarithms $[67,68]$

$$
\mathrm{Li}_{m_{k}, \ldots, m_{2}, m_{1}}\left(x_{k}, \ldots, x_{2}, x_{1}\right)=\sum_{i_{1}>i_{2}>\ldots>i_{k}>0}^{\infty} \frac{x_{1}^{i_{1}}}{i_{1}^{m_{1}}} \frac{x_{2}^{i_{2}}}{i_{2}^{m_{2}}} \ldots \frac{x_{k}^{i_{k}}}{i_{k}^{m_{k}}} .
$$

In the case with only one massive parton it is actually sufficient to restrict oneself to harmonic polylogarithms [69, 70], defined by

$$
H_{m_{1}, m_{2} \ldots, m_{k}}(x)=\sum_{i_{1}>i_{2}>\ldots>i_{k}>0}^{\infty} \frac{x^{i_{1}}}{i_{1}^{m_{1}}} \frac{1}{i_{2}^{m_{2}}} \ldots \frac{1}{i_{k}^{m_{k}}} .
$$

The additional dipole corresponding to gluon emission from a massive quark-antiquark system can be expressed in terms of two-dimensional harmonic polylogarithms [71].

\subsection{Dipole phase space measure in $\mathrm{D}$ dimensions}

In this section we derive the appropriate phase space measure for the dipoles with massive particles. Since singularities are regulated by dimensional regularization, this has 
to be done in $D$ dimensions. The phase space measure for $n$ particles in $D$ dimensions is given by [72]

$$
\begin{aligned}
d \phi_{n}\left(P \rightarrow p_{1}, \ldots, p_{n}\right) & =(2 \pi)^{D} \delta^{D}\left(P-\sum_{i=1}^{n} p_{i}\right) \prod_{i=1}^{n} \frac{d^{D} p_{i}}{(2 \pi)^{D-1}} \theta\left(p_{i}^{0}\right) \delta\left(p_{i}^{2}-m_{i}^{2}\right) \\
& =(2 \pi)^{D} \delta^{D}\left(P-\sum_{i=1}^{n} p_{i}\right) \prod_{i=1}^{n} \frac{d^{D-1} p_{i}}{(2 \pi)^{D-1} 2 E_{i}}
\end{aligned}
$$

with

$$
E_{i}=\sqrt{\left(\vec{p}_{i}\right)^{2}+m_{i}^{2}}
$$

The phase space measure factorizes according to

$$
d \phi_{n}\left(P \rightarrow p_{1}, \ldots, p_{n}\right)=\frac{1}{2 \pi} d \phi_{n-j+1}\left(P \rightarrow Q, p_{j+1}, \ldots, p_{n}\right) d Q^{2} d \phi_{j}\left(Q \rightarrow p_{1}, \ldots, p_{j}\right) .
$$

\subsubsection{Phase space measure with no initial-state particles}

We first evaluate the two particle phase space

$$
\int d \phi_{2}\left(P \rightarrow \tilde{p}_{i j}, \tilde{p}_{k}\right)=\int \frac{d^{D-1} \tilde{p}_{i j}}{(2 \pi)^{D-1} 2 E_{i j}} \frac{d^{D-1} \tilde{p}_{k}}{(2 \pi)^{D-1} 2 E_{k}}(2 \pi)^{D} \delta^{D}\left(P-\tilde{p}_{i j}-\tilde{p}_{k}\right)
$$

in the rest frame of $P$, e.g. $P=\left(\sqrt{P^{2}}, \overrightarrow{0}\right)$. We obtain

$$
\begin{aligned}
& \int d \phi_{2}\left(P \rightarrow \tilde{p}_{i j}, \tilde{p}_{k}\right) \\
& =(2 \pi)^{2-D}\left(\frac{1}{2}\right)^{D-1}\left(P^{2}\right)^{1-D / 2}\left(\sqrt{\left(P^{2}-m_{i j}^{2}-m_{k}^{2}\right)^{2}-4 m_{i j}^{2} m_{k}^{2}}\right)^{D-3} \int d \Omega_{D-1},
\end{aligned}
$$

where $\Omega_{D-1}$ parameterizes the solid angle of the $(D-1)$ spatial components of $\tilde{p}_{k}$ in $(D-1)$ (spatial) dimensions. With this convention we have

$$
\int d \Omega_{D}=\frac{2 \pi^{D / 2}}{\Gamma(D / 2)} .
$$

We then evaluate the three particle phase space

$$
\begin{aligned}
& \int d \phi_{3}\left(P \rightarrow p_{i}, p_{j}, p_{k}\right) \\
& \quad=\int \frac{d^{D-1} p_{i}}{(2 \pi)^{D-1} 2 E_{i}} \frac{d^{D-1} p_{j}}{(2 \pi)^{D-1} 2 E_{j}} \frac{d^{D-1} p_{k}}{(2 \pi)^{D-1} 2 E_{k}}(2 \pi)^{D} \delta^{D}\left(P-p_{i}-p_{j}-p_{k}\right)
\end{aligned}
$$


in the rest frame of $P$, e.g. $P=\left(\sqrt{P^{2}}, \overrightarrow{0}\right)$. We shall orient our frame such that the solid angle of the spatial components of $p_{k}$ coincides with the solid angle of the spatial components of $\tilde{p}_{k}$ in (3.28). It will be convenient to parameterize the spatial components of $p_{i}$ with spherical coordinates, using $\vec{p}_{k}$ as polar axis, e.g

$$
d^{D-1} p_{i}=\left|\vec{p}_{i}\right|^{D-2} d\left|\vec{p}_{i}\right| d \theta_{1} \sin ^{D-3} \theta_{1} d \Omega_{D-2}^{(i)}
$$

We therefore have

$$
2 \vec{p}_{i} \vec{p}_{k}=2\left|\vec{p}_{i}\right|\left|\vec{p}_{k}\right| \cos \theta_{1}
$$

Finally we obtain the three-particle phase space as the product of a two-particle phase space and a dipole phase space:

$$
\int d \phi_{3}\left(P \rightarrow p_{i}, p_{j}, p_{k}\right)=\int d \phi_{2}\left(P \rightarrow \tilde{p}_{i j}, \tilde{p}_{k}\right) d \phi_{\text {dipole }}\left(2 p_{i} p_{j}, 2 p_{j} p_{k}, 2 p_{i} p_{k}, \Omega_{D-2}^{(i)}\right),
$$

where

$$
\begin{gathered}
d \phi_{\text {dipole }}\left(2 p_{i} p_{j}, 2 p_{j} p_{k}, 2 p_{i} p_{k}\right)=(2 \pi)^{1-D} \frac{2 \pi^{D / 2-1}}{\Gamma\left(\frac{D}{2}-1\right)} \frac{1}{4}\left(\left(P^{2}-m_{i}^{2}-m_{k}^{2}\right)^{2}-4 m_{i}^{2} m_{k}^{2}\right)^{\frac{3-D}{2}} \\
\int d\left(2 p_{i} p_{j}\right) d\left(2 p_{j} p_{k}\right) d\left(2 p_{i} p_{k}\right) \delta\left(P^{2}-m_{i}^{2}-m_{j}^{2}-m_{k}^{2}-2 p_{i} p_{j}-2 p_{j} p_{k}-2 p_{i} p_{k}\right) \\
\left(-P^{2} \lambda\left(\left|\vec{p}_{i}\right|^{2},\left|\vec{p}_{j}\right|^{2},\left|\vec{p}_{k}\right|^{2}\right)\right)^{\frac{D-4}{2}} \theta\left(-\lambda\left(\left|\vec{p}_{i}\right|^{2},\left|\vec{p}_{j}\right|^{2},\left|\vec{p}_{k}\right|^{2}\right)\right)
\end{gathered}
$$

Note that we already performed the angular integration over $d \Omega_{D-2}^{(i)}$. The triangle function $\lambda$ is defined by

$$
\lambda(x, y, z)=x^{2}+y^{2}+z^{2}-2 x y-2 y z-2 z x .
$$

We further have

$$
\begin{aligned}
\left|\vec{p}_{i}\right|^{2} & =\frac{1}{4 P^{2}}\left(P^{2}+m_{i}^{2}-m_{k}^{2}-m_{j}^{2}-2 p_{j} p_{k}\right)^{2}-m_{i}^{2}, \\
\left|\vec{p}_{k}\right|^{2} & =\frac{1}{4 P^{2}}\left(P^{2}-m_{i}^{2}+m_{k}^{2}-m_{j}^{2}-2 p_{i} p_{j}\right)^{2}-m_{k}^{2} \\
\left|\vec{p}_{j}\right|^{2} & =\frac{1}{4 P^{2}}\left(P^{2}-m_{i}^{2}-m_{k}^{2}+m_{j}^{2}-2 p_{i} p_{k}\right)^{2}-m_{j}^{2}
\end{aligned}
$$

so that

$$
\begin{aligned}
& -P^{2} \lambda\left(\left|\vec{p}_{i}\right|^{2},\left|\vec{p}_{j}\right|^{2},\left|\vec{p}_{k}\right|^{2}\right) \\
& \quad=2 p_{i} p_{k} 2 p_{i} p_{j} 2 p_{j} p_{k}-m_{i}^{2}\left(2 p_{j} p_{k}\right)^{2}-m_{k}^{2}\left(2 p_{i} p_{j}\right)^{2}-m_{j}^{2}\left(2 p_{i} p_{k}\right)^{2}+4 m_{i}^{2} m_{j}^{2} m_{k}^{2} .
\end{aligned}
$$


To find a suitable parametrization of the dipole phase space, we treat the various cases separately. We consider first the case where two particles are massless $m_{i}=m_{j}=0$. With $D=4-2 \varepsilon$ the dipole phase space becomes

$$
\begin{aligned}
d \phi_{\text {dipole }}= & \frac{(4 \pi)^{\varepsilon-2}}{\Gamma(1-\varepsilon)}\left(P^{2}\right)^{1-\varepsilon}\left(u_{0}\right)^{2-2 \varepsilon} \\
& \cdot \int_{0}^{1} d u u^{1-2 \varepsilon}(1-u)^{1-2 \varepsilon}\left(1-u_{0} u\right)^{-1+\varepsilon} \int_{0}^{1} d v v^{-\varepsilon}(1-v)^{-\varepsilon}
\end{aligned}
$$

where

$$
\begin{aligned}
u_{0} & =\frac{P^{2}-m_{k}^{2}}{P^{2}} \\
u & =\frac{2 p_{i} p_{j}+2 p_{j} p_{k}}{2 p_{i} p_{k}+2 p_{i} p_{j}+2 p_{j} p_{k}}=\frac{2 p_{i} p_{j}+2 p_{j} p_{k}}{P^{2}-m_{k}^{2}} \\
v & =\frac{2 p_{i} p_{j}\left(2 p_{i} p_{k}+m_{k}^{2}\right)}{2 p_{i} p_{k}\left(2 p_{i} p_{j}+2 p_{j} p_{k}\right)}=\frac{2 p_{i} p_{j}\left(2 p_{i} p_{k}+m_{k}^{2}\right)}{2 p_{i} p_{k}\left(P^{2}-m_{k}^{2}-2 p_{i} p_{k}\right)} .
\end{aligned}
$$

In the case where one mass vanishes $\left(m_{j}=0\right)$ and the other two are equal $\left(m_{i}=m_{k}=\right.$ $m$ ) we obtain

$$
\begin{aligned}
d \phi_{\text {dipole }}= & \frac{(4 \pi)^{\varepsilon-2}}{\Gamma(1-\varepsilon)}\left(P^{2}\right)^{1-\varepsilon}\left(r_{0}\right)^{2-2 \varepsilon} 2^{2 \varepsilon-1} \\
& \cdot \int_{0}^{1} d r r^{1-2 \varepsilon}(1-r)^{-\varepsilon+1 / 2}\left(1-r_{0} r\right)^{-1 / 2} \int_{-1}^{1} d s\left(1-s^{2}\right)^{-\varepsilon}
\end{aligned}
$$

with

$$
\begin{aligned}
r_{0} & =1-4 \frac{m^{2}}{P^{2}}, \\
2 p_{i} p_{j} & =\frac{1}{2} P^{2} r_{0} r\left(1-s \sqrt{\frac{r_{0}(1-r)}{1-r_{0} r}}\right), \\
2 p_{j} p_{k} & =\frac{1}{2} P^{2} r_{0} r\left(1+s \sqrt{\frac{r_{0}(1-r)}{1-r_{0} r}}\right) .
\end{aligned}
$$

\subsubsection{Phase space involving initial state particles}

If initial state particles are involved, we obtain the following convolution:

$$
d \phi\left(p_{a}+p_{b} \rightarrow K+p_{k}+p_{i}\right)=\int_{0}^{1} d x d \phi\left(\tilde{p}_{a}+p_{b} \rightarrow K+\tilde{p}_{k}\right) d \phi_{\text {dipole }}
$$


with

$$
\tilde{p}_{a}=x p_{a}
$$

In the case $m_{a}=m_{i}=0$ we obtain

$d \phi_{\text {dipole }}=\frac{(4 \pi)^{\varepsilon-2}}{\Gamma(1-\varepsilon)}\left(-P^{2}\right)^{1-\varepsilon} x_{0}^{\varepsilon-1} x^{-1+\varepsilon}(1-x)^{1-2 \varepsilon}\left(1-x_{0} x\right)^{-1+\varepsilon} \int_{0}^{1} d w w^{-\varepsilon}(1-w)^{-\varepsilon}$

with $P=p_{k}+p_{i}-p_{a}$ and

$$
\begin{aligned}
x_{0} & =\frac{-P^{2}}{m_{k}^{2}-P^{2}}, \\
x & =\frac{2 p_{a} p_{i}+2 p_{a} p_{k}-2 p_{i} p_{k}}{2 p_{a} p_{i}+2 p_{a} p_{k}}, \\
w & =\frac{2 p_{a} p_{i}\left(2 p_{i} p_{k}+m_{k}^{2}\right)}{2 p_{i} p_{k}\left(2 p_{a} p_{i}+2 p_{a} p_{k}\right)} .
\end{aligned}
$$

Eq. (3.43) and eq. (3.45) are derived as follows: From the factorization of phase space we have

$$
d \phi\left(p_{a}+p_{b} \rightarrow K+p_{k}+p_{i}\right)=\frac{1}{2 \pi} d \phi\left(p_{a}+p_{b} \rightarrow K+Q\right) d m_{Q}^{2} d \phi\left(Q \rightarrow p_{i}+p_{k}\right) .
$$

We then derive

$$
d \phi\left(p_{a}+p_{b} \rightarrow K+Q\right)=x d \phi\left(\tilde{p}_{a}+p_{b} \rightarrow K+\tilde{p}_{k}\right) .
$$

This equation can be obtained by writing out the explicit expressions for the phase space measures in the rest frame of $p_{a}+p_{b}$ and $\tilde{p}_{a}+p_{b}$, respectively. These two frames are related by a boost. Further, since $\tilde{p}_{a}=x p_{a}$, the boost does not affect the transverse components. Next, one writes out the parameterization for $d \phi\left(Q \rightarrow p_{i}+p_{k}\right)$ in terms of solid angles of particle $p_{k}$, singles out one angle $\theta_{1}=\angle\left(p_{k}, p_{a}\right)$ and replaces the integrations over $d m_{Q}^{2} d \cos \theta_{1}$ by integrations over $d x d w$.

\subsection{Dipole terms with no initial-state partons}

In this section we give the dipole factors corresponding to the case where all relevant partons are in the final state. Initial-state partons are considered in the next section. We distinguish the cases of (i) a massless emitter and massive spectator, (ii) a massive 
emitter and a massless spectator and (iii) a massive emitter and a massive spectator of equal mass. We follow closely the notation of Catani and Seymour [45].

The generic form of the dipole terms is given by

$$
\mathcal{D}_{i j, k}=-\frac{1}{2 p_{i} \cdot p_{j}}\left\langle 1, \ldots,(\tilde{i j}), \ldots, \tilde{k}, \ldots\left|\frac{\mathbf{T}_{k} \cdot \mathbf{T}_{i j}}{\mathbf{T}_{i j}^{2}} V_{i j, k}\right| 1, \ldots,(\tilde{i j}), \ldots, \tilde{k}, \ldots\right\rangle .
$$

The explicit forms of the functions $V_{i j, k}$ are given below.

\subsubsection{Massless final emitter, massive final spectator}

We consider first the case of massless emitter (particle $i$ ) and a massive spectator (particle $k$ ), both in the final state. The variables $y$ and $z$ of Catani and Seymour are given by

$$
y=\frac{2 p_{i} p_{j}}{2 p_{i} p_{j}+2 p_{j} p_{k}+2 p_{i} p_{k}}, \quad z=\frac{2 p_{i} p_{k}}{2 p_{i} p_{k}+2 p_{j} p_{k}}
$$

and related to the variables $u$ and $v$ as follows:

$$
\begin{aligned}
y & =u_{0} v \frac{u(1-u)}{1-u_{0} u}, \quad u_{0}=1-\frac{m_{k}^{2}}{\left(p_{i}+p_{j}+p_{k}\right)^{2}} \\
z(1-y) & =1-u .
\end{aligned}
$$

In the collinear limit eq.(3.14) we have

$$
y \rightarrow 0, \quad z \rightarrow \hat{z}
$$

and in the soft limit $p_{j} \rightarrow 0$

$$
y \rightarrow 0, \quad z \rightarrow 1
$$

In addition, the subtraction term for the splitting $g \rightarrow g g$ has to match the soft limit $p_{i} \rightarrow 0$, corresponding to $y \rightarrow 0, z \rightarrow 0$.

As subtraction terms we use

$$
\begin{aligned}
\left\langle s\left|V_{q_{i} g_{j}, k}\right| s^{\prime}\right\rangle= & 8 \pi \mu^{2 \varepsilon} \alpha_{s} C_{F} \delta_{s s^{\prime}}\left[\frac{2 z(1-y)}{1-z(1-y)}+(1-\rho \varepsilon)(1-z(1-y))\right], \\
\left\langle\mu\left|V_{q_{i} \bar{q}_{j}, k}\right| \nu\right\rangle= & 8 \pi \mu^{2 \varepsilon} \alpha_{s} T_{R}\left[-g^{\mu \nu}-\frac{4}{2 p_{i} p_{j}} S^{\mu \nu}\right], \\
\left\langle\mu\left|V_{g_{i} g_{j}, k}\right| \nu\right\rangle= & 16 \pi \mu^{2 \varepsilon} \alpha_{s} C_{A}\left[-g^{\mu \nu}\left(\frac{z(1-y)}{1-z(1-y)}+\frac{(1-z)(1-y)}{1-(1-z)(1-y)}\right)\right. \\
& \left.+(1-\rho \varepsilon) \frac{2}{2 p_{i} p_{j}} S^{\mu \nu}\right]
\end{aligned}
$$


where the spin correlation tensor is given by

$$
S^{\mu \nu}=\left(z(1-y) p_{i}^{\mu}-(1-z(1-y)) p_{j}^{\mu}\right)\left(z(1-y) p_{i}^{\nu}-(1-z(1-y)) p_{j}^{\nu}\right) .
$$

The momenta are mapped as follows:

$$
\begin{aligned}
& \tilde{p}_{i}=a p_{i}+b p_{j}+c p_{k}, \\
& \tilde{p}_{k}=(1-a) p_{i}+(1-b) p_{j}+(1-c) p_{k},
\end{aligned}
$$

with

$$
\begin{aligned}
c & =\frac{y u_{0}}{(2 u-1-y(1-u))^{2} u_{0}+4 u(1-u) y}\left(2 u(1-u)-\frac{N}{\sqrt{1-v}}\right) \\
b & =\frac{1}{N}\left\{u+\frac{c}{y u_{0}}\left[-2 y u-u_{0}\left(\left(1-2 u-u^{2}\right) y+1-3 u+2 u^{2}\right)\right]\right\} \\
a & =\frac{1}{N}\left\{1-u-\frac{c}{y u_{0}}\left[2 y(1-u)-u_{0}\left((1-u) y^{2}+\left(1-u+u^{2}\right) y+u-2 u^{2}\right)\right]\right\} \\
N & =u^{2}+(1-u)^{2}+(1-u) y .
\end{aligned}
$$

This mapping satisfies momentum conservation

$$
\tilde{p}_{i}+\tilde{p}_{k}=p_{i}+p_{j}+p_{k},
$$

and the on-shell conditions

$$
\tilde{p}_{i}^{2}=0, \quad \tilde{p}_{k}^{2}=m_{k}^{2}
$$

In the limit $y \rightarrow 0$ we have

$$
\lim _{y \rightarrow 0} \tilde{p}_{i}=p_{i}+p_{j}, \quad \lim _{y \rightarrow 0} \tilde{p}_{k}=p_{k} .
$$

In addition we have

$$
S_{\mu \nu} \tilde{p}_{i}^{\nu}=0
$$

e.g. $\tilde{p}_{i}$ is orthogonal to the spin correlation tensor. The integral over the spin correlation can be written as

$$
\int d \phi_{\text {dipole }} \frac{-2}{\left(2 p_{i} p_{j}\right)^{2}} S^{\mu \nu}=C_{21} \tilde{p}_{i}^{\mu} \tilde{p}_{i}^{\nu}+C_{22} \tilde{p}_{k}^{\mu} \tilde{p}_{k}^{\nu}+C_{23}\left(\tilde{p}_{i}^{\mu} \tilde{p}_{k}^{\nu}+\tilde{p}_{k}^{\mu} \tilde{p}_{i}^{\nu}\right)+C_{24} g^{\mu \nu} .
$$

Using $S_{\mu \nu} \tilde{p}_{i}^{\nu}=0$ and $\tilde{p}_{i}^{2}=0$ this reduces to

$$
C_{21} \tilde{p}_{i}^{\mu} \tilde{p}_{i}^{\nu}-C_{24}\left(-g^{\mu \nu}+2 \frac{\tilde{p}_{i}^{\mu} \tilde{p}_{k}^{\nu}+\tilde{p}_{k}^{\mu} \tilde{p}_{i}^{\nu}}{2 \tilde{p}_{i} \tilde{p}_{k}}\right) .
$$


Due to gauge invariance only the term $-C_{24}\left(-g^{\mu \nu}\right)$ will give a non-vanishing contribution. $C_{24}$ is obtained by contracting with $g_{\mu \nu}$ :

$$
C_{24}=\frac{1}{2(1-\rho \varepsilon)} \int d \phi_{\text {dipole }} \frac{-2}{\left(2 p_{i} p_{j}\right)^{2}} g_{\mu \nu} S^{\mu \nu}
$$

Integration of the subtraction terms yields:

$$
\begin{aligned}
& \mathcal{V}_{q_{i} g_{j}, k}=\int d \phi_{\text {dipole }} \frac{1}{2 p_{i} p_{j}} V_{q_{i} g_{j}, k}=\frac{\alpha_{s}}{2 \pi} \frac{1}{\Gamma(1-\varepsilon)}\left(\frac{4 \pi \mu^{2} P^{2}}{\left(P^{2}-m_{k}^{2}\right)^{2}}\right)^{\varepsilon} \mathcal{V}_{q g}\left(u_{0}, \varepsilon\right), \\
& \mathcal{V}_{q g}\left(u_{0}, \varepsilon\right) \\
& =C_{F} \delta_{s s^{\prime}} \int_{0}^{1} d u \int_{0}^{1} d v u^{-2 \varepsilon}(1-u)^{-2 \varepsilon}\left(1-u_{0} u\right)^{\varepsilon} v^{-1-\varepsilon}(1-v)^{-\varepsilon} \\
& {\left[2 \frac{1-u}{u}+(1-\rho \varepsilon) u\right]} \\
& =C_{F} \delta_{s s^{\prime}} \frac{\Gamma(-\varepsilon) \Gamma(1-\varepsilon)}{\Gamma(1-2 \varepsilon)} \Gamma(2-2 \varepsilon)\left[2 \frac{\Gamma(-2 \varepsilon)}{\Gamma(2-4 \varepsilon)}{ }_{2} F_{1}\left(-\varepsilon,-2 \varepsilon ; 2-4 \varepsilon ; u_{0}\right)\right. \\
& \left.+(1-\rho \varepsilon) \frac{\Gamma(1-2 \varepsilon)}{\Gamma(3-4 \varepsilon)}{ }_{2} F_{1}\left(-\varepsilon, 2-2 \varepsilon ; 3-4 \varepsilon ; u_{0}\right)\right] \\
& =C_{F} \delta_{s s^{\prime}}\left[\frac{1}{\varepsilon^{2}}+\frac{3}{2 \varepsilon}+\frac{17}{4}+\frac{1}{2} \rho-\frac{5}{6} \pi^{2}+\frac{1}{2 u_{0}}+\frac{1}{2} \frac{\left(1-u_{0}\right)\left(1-3 u_{0}\right)}{u_{0}^{2}} \ln \left(1-u_{0}\right)\right. \\
& \left.+2 \mathrm{Li}_{2}\left(u_{0}\right)\right]+O(\varepsilon), \\
& \mathcal{V}_{q_{i} \bar{q}_{j}, k}=\int d \phi_{\text {dipole }} \frac{1}{2 p_{i} p_{j}} V_{q_{i} \bar{q}_{j}, k}=\frac{\alpha_{s}}{2 \pi} \frac{1}{\Gamma(1-\varepsilon)}\left(\frac{4 \pi \mu^{2} P^{2}}{\left(P^{2}-m_{k}^{2}\right)^{2}}\right)^{\varepsilon} \mathcal{V}_{q \bar{q}}\left(u_{0}, \varepsilon\right) \text {, } \\
& \mathcal{V}_{q \bar{q}}\left(u_{0}, \varepsilon\right) \\
& =\int_{0}^{1} d u \int_{0}^{1} d v u^{-2 \varepsilon}(1-u)^{-2 \varepsilon}\left(1-u_{0} u\right)^{\varepsilon} v^{-1-\varepsilon}(1-v)^{-\varepsilon} T_{R}\left[-g^{\mu \nu}-\frac{4}{2 p_{i} p_{j}} S^{\mu \nu}\right] \\
& =T_{R}\left(-g^{\mu \nu}\right) \frac{\Gamma(-\varepsilon) \Gamma(1-\varepsilon)}{\Gamma(1-2 \varepsilon)}\left[\frac{\Gamma(1-2 \varepsilon)^{2}}{\Gamma(2-4 \varepsilon)}{ }_{2} F_{1}\left(-\varepsilon, 1-2 \varepsilon, 2-4 \varepsilon, u_{0}\right)\right. \\
& \left.-\frac{2}{1-\rho \varepsilon} \frac{\Gamma(2-2 \varepsilon)^{2}}{\Gamma(4-4 \varepsilon)}{ }_{2} F_{1}\left(-\varepsilon, 2-2 \varepsilon, 4-4 \varepsilon, u_{0}\right)\right]+ \text { gauge terms } \\
& =T_{R}\left(-g^{\mu \nu}\right)\left[-\frac{2}{3 \varepsilon}-\frac{13}{6}+\frac{1}{3} \rho+\frac{2}{3} \frac{\left(1-u_{0}\right)}{u_{0}^{2}}\right. \\
& \left.+\frac{1}{3} \frac{\left(1-u_{0}\right)}{u_{0}^{3}}\left(2 u_{0}^{2}-u_{0}+2\right) \ln \left(1-u_{0}\right)\right]+ \text { gauge terms }+O(\varepsilon), \\
& \mathcal{V}_{g_{i} g_{j}, k}=\int d \phi_{\text {dipole }} \frac{1}{2 p_{i} p_{j}} V_{g_{i} g_{j}, k}=\frac{\alpha_{s}}{2 \pi} \frac{1}{\Gamma(1-\varepsilon)}\left(\frac{4 \pi \mu^{2} P^{2}}{\left(P^{2}-m_{k}^{2}\right)^{2}}\right)^{\varepsilon} \mathcal{V}_{g g}\left(u_{0}, \varepsilon\right)
\end{aligned}
$$




$$
\begin{aligned}
\mathcal{V}_{g g}\left(u_{0}, \varepsilon\right) & \\
= & 2 C_{A} \int_{0}^{1} d u \int_{0}^{1} d v u^{-2 \varepsilon}(1-u)^{-2 \varepsilon}\left(1-u_{0} u\right)^{\varepsilon} v^{-1-\varepsilon}(1-v)^{-\varepsilon} \\
& \cdot\left[-g^{\mu \nu}\left(\frac{1-u}{u}+\frac{u}{1-u}-\frac{u}{(1-u)} \frac{u_{0} v}{\left(1-u_{0} u(1-v)\right)}\right)+(1-\rho \varepsilon) \frac{2}{2 p_{i} p_{j}} S^{\mu \nu}\right] \\
= & 2 C_{A}\left(-g^{\mu \nu}\right) \frac{\Gamma(-\varepsilon) \Gamma(1-\varepsilon)}{\Gamma(1-2 \varepsilon)}\left[\frac { \Gamma ( - 2 \varepsilon ) \Gamma ( 2 - 2 \varepsilon ) } { \Gamma ( 2 - 4 \varepsilon ) } \left({ }_{2} F_{1}\left(-\varepsilon,-2 \varepsilon, 2-4 \varepsilon, u_{0}\right)\right.\right. \\
& \left.\left.+{ }_{2} F_{1}\left(-\varepsilon, 2-2 \varepsilon, 2-4 \varepsilon, u_{0}\right)\right)+\frac{\Gamma(2-2 \varepsilon)^{2}}{\Gamma(4-4 \varepsilon)}{ }_{2} F_{1}\left(-\varepsilon, 2-2 \varepsilon, 4-4 \varepsilon, u_{0}\right)\right] \\
& +2 C_{A}\left(-g^{\mu \nu}\right)\left(-u_{0}\right) \frac{\Gamma(1-\varepsilon) \Gamma(-2 \varepsilon)}{\Gamma(-\varepsilon)} \\
& \sum_{n=0}^{\infty} \sum_{m=0}^{\infty} \frac{\Gamma(n+1-\varepsilon)}{\Gamma(n+2-2 \varepsilon)} \frac{\Gamma(m+n+2-2 \varepsilon)}{\Gamma(m+n+2-4 \varepsilon)} \frac{\Gamma(m-\varepsilon)}{m !} u_{0}^{n+m}+\text { gauge terms } \\
= & 2 C_{A}\left(-g^{\mu \nu}\right)\left[\frac{1}{\varepsilon^{2}}+\frac{11}{6} \frac{1}{\varepsilon}-\frac{5}{6} \pi^{2}+\frac{67}{12}-\frac{1}{3} \frac{\left(1-u_{0}\right)}{u_{0}^{2}}+2 \operatorname{Li}_{2}\left(u_{0}\right)\right. \\
& \left.-\frac{1}{6}\left(\frac{\left(1-u_{0}\right)}{u_{0}^{3}}\left(2-u_{0}\right)+11 \frac{\left(1-u_{0}\right)}{u_{0}}\right) \ln \left(1-u_{0}\right)\right] \\
+ & \text { gauge terms }+O(\varepsilon) .
\end{aligned}
$$

\subsubsection{Massive final emitter, massless final spectator}

We now consider the case of a massive emitter (particle $k$ ) and a massless spectator (particle $i$ ). It is sufficient to consider the case where a heavy quark (or antiquark) emits a gluon. We now have

$$
y=\frac{2 p_{j} p_{k}}{2 p_{i} p_{j}+2 p_{j} p_{k}+2 p_{i} p_{k}}, \quad z=\frac{2 p_{i} p_{k}}{2 p_{i} p_{k}+2 p_{i} p_{j}}
$$

and

$$
\begin{aligned}
y & =u\left(1-\frac{(1-u) u_{0}}{1-u_{0} u} v\right), \quad u_{0}=1-\frac{m_{k}^{2}}{\left(p_{i}+p_{j}+p_{k}\right)^{2}} \\
z(1-y) & =1-u .
\end{aligned}
$$

The momenta are mapped as follows:

$$
\begin{aligned}
\text { emitter : } & \tilde{p}_{k}=p_{k}+p_{j}-\frac{y}{1-y} p_{i}, \\
\text { spectator : } & \tilde{p}_{i}=\frac{1}{1-y} p_{i} .
\end{aligned}
$$


Since there is no collinear singularity, we just have to match the part of the soft singularity which corresponds to this dipole factor:

$$
\left\langle s\left|V_{q_{k} g_{j}, i}\right| s^{\prime}\right\rangle=8 \pi \mu^{2 \varepsilon} \alpha_{s} C_{F} \delta_{s s^{\prime}}\left[\frac{2 z(1-y)}{1-z(1-y)}-2\left(\frac{1-u_{0}}{u_{0}}\right) \frac{1}{y}\right] .
$$

With

$$
v_{0}=\frac{u_{0}(1-u)}{1-u_{0} u}
$$

we obtain for the integral over the dipole phase space

$$
\mathcal{V}_{q_{k} g_{j}, i}=\int d \phi_{\text {dipole }} \frac{1}{2 p_{j} p_{k}} V_{q_{k} g_{j}, i}=\frac{\alpha_{s}}{2 \pi} \frac{1}{\Gamma(1-\varepsilon)}\left(\frac{4 \pi \mu^{2} P^{2}}{\left(P^{2}-m_{k}\right)^{2}}\right)^{\varepsilon} \mathcal{V}_{Q g}\left(u_{0}, \varepsilon\right)
$$

$$
\begin{aligned}
\mathcal{V}_{Q g}\left(u_{0}, \varepsilon\right)= & C_{F} \delta_{s s^{\prime}} \cdot 2 \int_{0}^{1} d u \int_{0}^{1} d v u^{-2 \varepsilon-1}(1-u)^{1-2 \varepsilon}\left(1-u_{0} u\right)^{\varepsilon-1} \\
& v^{-\varepsilon}(1-v)^{-\varepsilon}\left(1-v_{0} v\right)^{-1}\left[u_{0}(1-u)-\left(1-u_{0}\right)\left(1-v_{0} v\right)^{-1}\right] \\
= & C_{F} \delta_{s s^{\prime}} \cdot 2 \Gamma(1-\varepsilon) \sum_{n=0}^{\infty} \sum_{m=0}^{\infty} \frac{\Gamma(m-2 \varepsilon) \Gamma(n+m+1-\varepsilon)}{\Gamma(n+m+2-4 \varepsilon)} \\
& \cdot\left(\frac{n+2-2 \varepsilon}{n+m+2-4 \varepsilon} u_{0}-(n+1)\left(1-u_{0}\right)\right) \frac{u_{0}^{n+m}}{m !} \\
= & C_{F} \delta_{s s^{\prime}}\left[\frac{1}{\varepsilon}\left(1+\ln \left(1-u_{0}\right)\right)+4+\ln \left(1-u_{0}\right)-4 \operatorname{Li}_{2}\left(u_{0}\right)-\frac{1}{2} \ln ^{2}\left(1-u_{0}\right)\right] \\
& +O(\varepsilon) .
\end{aligned}
$$

Here we used

$$
\begin{aligned}
& \sum_{i_{1}=2}^{\infty} \sum_{i_{2}=1}^{i_{1}-1} \frac{u_{0}^{i_{1}}}{i_{1}} \frac{1}{i_{2}}=\operatorname{Li}_{1,1}\left(1, u_{0}\right)=H_{11}\left(u_{0}\right)=\frac{1}{2} \ln ^{2}\left(1-u_{0}\right), \\
& \sum_{i_{1}=2}^{\infty} \sum_{i_{2}=1}^{i_{1}-1} u_{0}^{i_{1}} \frac{1}{i_{2}}=\operatorname{Li}_{1,0}\left(1, u_{0}\right)=-\frac{u_{0}}{1-u_{0}} \ln \left(1-u_{0}\right) .
\end{aligned}
$$

\subsubsection{Massive final emitter and massive final spectator of equal mass}

We now consider the case of a massive emitter (particle $i$ ) and a massive spectator (particle $k$ ) of equal mass $\left(m_{i}=m_{k}=m\right)$. It is sufficient to consider the case where 
a heavy quark (or antiquark) emits a gluon. Since there is no collinear singularity, we just have to match the part of the soft singularity which corresponds to this dipole factor:

$$
\begin{aligned}
& \left\langle s\left|V_{q_{i} g_{j}, k}\right| s^{\prime}\right\rangle \\
& =8 \pi \mu^{2 \varepsilon} \alpha_{s} C_{F} \delta_{s s^{\prime}} \sqrt{\frac{\left(2 p_{i} p_{j}+2 p_{j} p_{k}+2 p_{i} p_{k}\right)^{2}-\left(2 m^{2}\right)^{2}}{\left(2 p_{i} p_{k}\right)^{2}-\left(2 m^{2}\right)^{2}}}\left[2 \frac{2 p_{i} p_{k}}{2 p_{i} p_{j}+2 p_{j} p_{k}}-2 \frac{m^{2}}{2 p_{i} p_{j}}\right] \\
& =8 \pi \mu^{2 \varepsilon} \alpha_{s} C_{F} \delta_{s s^{\prime}} \frac{1}{r_{0} r \sqrt{(1-r)\left(1-r_{0} r\right)}}\left[2\left(1-r_{0} r\right)-\left(1-r_{0}\right)-\frac{1-r_{0}}{1-s_{0} s}\right],
\end{aligned}
$$

where

$$
\begin{aligned}
r & =\frac{2 p_{i} p_{j}+2 p_{j} p_{k}}{2 p_{i} p_{j}+2 p_{j} p_{k}+2 p_{i} p_{k}-2 m^{2}} \\
s & =-\frac{1}{s_{0}} \frac{2 p_{i} p_{j}-2 p_{j} p_{k}}{2 p_{i} p_{j}+2 p_{j} p_{k}} \\
s_{0} & =\sqrt{\frac{r_{0}(1-r)}{1-r_{0} r}}, \quad r_{0}=1-\frac{4 m^{2}}{\left(p_{i}+p_{j}+p_{k}\right)^{2}} .
\end{aligned}
$$

The singularity occurs for $r \rightarrow 0$. In this limit, the expression in the square root tends to 1 . The inclusion of the square root term facilitates the analytic integration of the dipole term. The momenta are mapped as follows:

$$
\begin{aligned}
& \tilde{p}_{i}=\frac{1}{2} P-\sqrt{\frac{r_{0}}{y_{0}^{2}-\left(1-r_{0}\right)}}\left(p_{k}-\frac{y_{0}}{2} P\right), \\
& \tilde{p}_{k}=\frac{1}{2} P+\sqrt{\frac{r_{0}}{y_{0}^{2}-\left(1-r_{0}\right)}}\left(p_{k}-\frac{y_{0}}{2} P\right),
\end{aligned}
$$

with $P=p_{i}+p_{j}+p_{k}$ and

$$
y_{0}=\frac{2 p_{i} p_{k}+2 p_{j} p_{k}+2 m^{2}}{P^{2}}
$$

This mapping satisfies the on-shell conditions $\tilde{p}_{i}^{2}=\tilde{p}_{k}^{2}=m^{2}$. In the soft limit $y_{0}$ tends to 1 and the correct asymptotic behavior of the mapping is easily verified. Integration yields:

$$
\mathcal{V}_{q_{i} g_{j}, k}=\int d \phi_{\text {dipole }} \frac{1}{2 p_{i} p_{j}} V_{q_{i} g_{j}, k}=\frac{\alpha_{s}}{2 \pi} \frac{1}{\Gamma(1-\varepsilon)}\left(\frac{4 \pi \mu^{2}}{P^{2}}\right)^{\varepsilon} \mathcal{V}_{Q Q}\left(r_{0}, \varepsilon\right)
$$




$$
\begin{aligned}
& \mathcal{V}_{Q Q}\left(r_{0}, \varepsilon\right)=r_{0}^{-2 \varepsilon} 2^{2 \varepsilon} \int_{0}^{1} d r r^{-2 \varepsilon-1}(1-r)^{-\varepsilon}\left(1-r_{0} r\right)^{-1} \int_{-1}^{1} d s\left(1-s^{2}\right)^{-\varepsilon} \\
& C_{F} \delta_{s s^{\prime}}\left[\left(2\left(1-r_{0} r\right)-\left(1-r_{0}\right)\right)\left(1-s_{0} s\right)^{-1}-\left(1-r_{0}\right)\left(1-s_{0} s\right)^{-2}\right] \\
& =C_{F} \delta_{s s^{\prime}} \frac{1}{1-\varepsilon} 2^{2 \varepsilon} r_{0}^{-2 \varepsilon} \sum_{i=0}^{\infty} \sum_{j=0}^{\infty} \sum_{k=0}^{\infty}(-1)^{i}\left(1+(-1)^{j}\right) r_{0}^{k+j / 2} \\
& \frac{\Gamma(1+i+j) \Gamma(2-\varepsilon)}{\Gamma(2+i+j-\varepsilon)} \frac{\Gamma(i+\varepsilon)}{\Gamma(\varepsilon) i !} \frac{\Gamma(k+j / 2) \Gamma(k-2 \varepsilon) \Gamma(1+j / 2-\varepsilon)}{\Gamma(j / 2) \Gamma(k+j / 2+1-3 \varepsilon) k !} \\
& {\left[2-\left(1-r_{0}\right)(2+j) \frac{j+2 k}{j}\right]} \\
& =C_{F} \delta_{s s^{\prime}}\left\{\frac{1}{\varepsilon}\left(1-\frac{1}{2} \frac{1+r_{0}}{\sqrt{r_{0}}} \ln \frac{1+\sqrt{r_{0}}}{1-\sqrt{r_{0}}}\right)-2 \ln r_{0}\right. \\
& -\ln ^{2}\left(\frac{1+\sqrt{r_{0}}}{1-\sqrt{r_{0}}}\right)+\frac{1}{\sqrt{r_{0}}} \ln \left(\frac{1+\sqrt{r_{0}}}{1-\sqrt{r_{0}}}\right) \\
& -\frac{1+r_{0}}{2 \sqrt{r_{0}}}\left(\operatorname{Li}_{2}\left(\sqrt{r_{0}}\right)-\operatorname{Li}_{2}\left(-\sqrt{r_{0}}\right)+2 \operatorname{Li}_{2}\left(\frac{1+\sqrt{r_{0}}}{2}\right)\right. \\
& -2 \operatorname{Li}_{2}\left(\frac{1-\sqrt{r_{0}}}{2}\right)+\operatorname{Li}_{2}\left(\frac{\sqrt{r_{0}}-1}{2 \sqrt{r_{0}}}\right)-\operatorname{Li}_{2}\left(\frac{\sqrt{r_{0}}-1}{\sqrt{r_{0}}}\right) \\
& +\mathrm{Li}_{2}\left(\frac{1}{1+\sqrt{r_{0}}}\right)-\mathrm{Li}_{2}\left(\frac{1-\sqrt{r_{0}}}{1+\sqrt{r_{0}}}\right) \\
& -2 \ln r_{0} \ln \left(\frac{1+\sqrt{r_{0}}}{1-\sqrt{r_{0}}}\right)+\ln 2 \ln \frac{\sqrt{r_{0}}}{1+\sqrt{r_{0}}}+\frac{1}{2} \ln ^{2} 2 \\
& \left.\left.+\ln \left(1-\sqrt{r_{0}}\right) \ln \left(\frac{1+\sqrt{r_{0}}}{\sqrt{r_{0}}}\right)+\frac{1}{2} \ln ^{2}\left(1+\sqrt{r_{0}}\right)-\frac{1}{2} \ln ^{2}\left(1-\sqrt{r_{0}}\right)\right)\right\} \\
& +O(\varepsilon) .
\end{aligned}
$$

Here we used

$$
\begin{aligned}
& \sum_{i_{1}=2}^{\infty} \sum_{i_{2}=1}^{i_{1}-1} \frac{x_{1}^{i_{1}}}{i_{1}} \frac{x_{2}^{i_{2}}}{i_{2}}=\operatorname{Li}_{1,1}\left(x_{2}, x_{1}\right) \\
& =\ln \left(1-x_{1}\right) \ln \left(1-x_{2}\right)+\operatorname{Li}_{2}\left(-\frac{x_{2}}{1-x_{2}}\right)-\operatorname{Li}_{2}\left(-\frac{x_{2}\left(1-x_{1}\right)}{1-x_{2}}\right) \\
& =\quad-\frac{1}{2} \ln ^{2}\left(1-x_{1} x_{2}\right)+\ln \left(x_{2}\left(1-x_{1}\right)\right) \ln \left(1-x_{1} x_{2}\right)-\operatorname{Li}_{2}\left(\frac{1-x_{2}}{1-x_{1} x_{2}}\right) \\
& \quad+\operatorname{Li}_{2}\left(1-x_{2}\right), \\
& \sum_{i_{1}=2}^{\infty} \sum_{i_{2}=1}^{i_{1}-1} x_{1}^{i_{1}} \frac{x_{2}^{i_{2}}}{i_{2}}=\operatorname{Li}_{1,0}\left(x_{2}, x_{1}\right)=-\frac{x_{1}}{1-x_{1}} \ln \left(1-x_{1} x_{2}\right),
\end{aligned}
$$




$$
\sum_{i_{1}=2}^{\infty} \sum_{i_{2}=1}^{i_{1}-1} \frac{x_{1}^{i_{1}}}{i_{1}} x_{2}^{i_{2}}=\operatorname{Li}_{0,1}\left(x_{2}, x_{1}\right)=\frac{1}{1-x_{2}} \ln \left(1-x_{1} x_{2}\right)-\frac{x_{2}}{1-x_{2}} \ln \left(1-x_{1}\right) .
$$

\subsection{Dipole terms with initial-state partons}

In this section we consider initial-state partons. We distinguish the cases of (i) a massless emitter in the initial state and massive spectator in the final state, (ii) a massive emitter in the final state and a massless spectator in the initial state. The generic form of the dipole terms for case (i) is given by

$$
\mathcal{D}_{k}^{a i}=-\frac{1}{2 p_{a} \cdot p_{i}} \frac{1}{x}\left\langle 1, \ldots,(\tilde{a i}), \ldots, \tilde{k}, \ldots\left|\frac{\mathbf{T}_{k} \cdot \mathbf{T}_{a i}}{\mathbf{T}_{a i}^{2}} V_{k}^{a i}\right| 1, \ldots,(\tilde{a i}), \ldots, \tilde{k}, \ldots\right\rangle .
$$

In case (ii) we have

$$
\mathcal{D}_{k i}^{a}=-\frac{1}{2 p_{i} \cdot p_{k}} \frac{1}{x}\left\langle 1, \ldots,(\tilde{k i}), \ldots, \tilde{a}, \ldots\left|\frac{\mathbf{T}_{a} \cdot \mathbf{T}_{k i}}{\mathbf{T}_{k i}^{2}} V_{k i}^{a}\right| 1, \ldots,(\tilde{k} i), \ldots, \tilde{a}, \ldots\right\rangle .
$$

The explicit forms of the functions $V_{k}^{a i}$ and $V_{k i}^{a}$ are given below.

\subsubsection{Massless initial emitter, massive final spectator}

We now consider the case of a massless emitter (particle $a$ ) in the initial state and a massive spectator (particle $k$ ) in the final state. The variables $u$ and $x$ of Catani and Seymour are given by

$$
u=\frac{2 p_{i} p_{a}}{2 p_{i} p_{a}+2 p_{k} p_{a}}, \quad x=\frac{2 p_{i} p_{a}+2 p_{k} p_{a}-2 p_{i} p_{k}}{2 p_{i} p_{a}+2 p_{k} p_{a}} .
$$

The variables $u$ and $w$ are related by

$$
u=\frac{1-x}{1-x_{0} x} w, \quad x_{0}=1-\frac{m_{k}^{2}}{m_{k}^{2}-\left(p_{k}+p_{i}-p_{a}\right)^{2}} .
$$

As subtraction terms we use

$$
\begin{aligned}
\left\langle s\left|V_{k}^{q_{a} g_{i}}\right| s^{\prime}\right\rangle= & 8 \pi \mu^{2 \varepsilon} \alpha_{s} C_{F} \delta_{s s^{\prime}}\left[\frac{2}{1-x+u}-(1+x)-\rho \varepsilon(1-x)\right], \\
\left\langle s\left|V_{k}^{g_{a} \bar{q}_{i}}\right| s^{\prime}\right\rangle= & 8 \pi \mu^{2 \varepsilon} \alpha_{s} T_{R} \delta_{s s^{\prime}}[1-\rho \varepsilon-2 x(1-x)], \\
\left\langle\mu\left|V_{k}^{q_{a} q_{i}}\right| \nu\right\rangle= & 8 \pi \mu^{2 \varepsilon} \alpha_{s} C_{F}\left[-g^{\mu \nu} x+4 \frac{(1-x)}{x} \frac{r(1-r)}{2 p_{i} q} S^{\mu \nu}\right], \\
\left\langle\mu\left|V_{k}^{g_{a} g_{i}}\right| \nu\right\rangle= & 16 \pi \mu^{2 \varepsilon} \alpha_{s} C_{A}\left[-g^{\mu \nu}\left(\frac{1}{1-x+u}-1+x(1-x)\right)\right. \\
& \left.+2(1-\rho \varepsilon) \frac{(1-x)}{x} \frac{r(1-r)}{2 p_{i} q} S^{\mu \nu}\right]
\end{aligned}
$$


with

$$
S^{\mu \nu}=\left(\frac{1}{r} p_{i}^{\mu}-\frac{1}{1-r} q^{\mu}\right)\left(\frac{1}{r} p_{i}^{\nu}-\frac{1}{1-r} q^{\nu}\right) .
$$

Here $q$ is an arbitrary null vector not equal to $p_{i}$ and

$$
r=\frac{2 p_{i} p_{a}}{2 p_{i} p_{a}+2 q p_{a}} .
$$

The momenta are mapped as follows:

$$
\begin{aligned}
& \tilde{p}_{a}=x p_{a} \\
& \tilde{p}_{k}=p_{k}+p_{i}-(1-x) p_{a} .
\end{aligned}
$$

This mapping satisfies momentum conservation

$$
\tilde{p}_{k}-\tilde{p}_{a}=p_{k}+p_{i}-p_{a},
$$

and the on-shell conditions

$$
\tilde{p}_{a}^{2}=0, \quad \tilde{p}_{k}^{2}=m_{k}^{2} .
$$

The desired asymptotic behavior

$$
\lim _{u \rightarrow 0} \tilde{p}_{a}=x p_{a}, \quad \lim _{u \rightarrow 0} \tilde{p}_{k}=p_{k}
$$

is fulfilled. In addition we have

$$
S_{\mu \nu} \tilde{p}_{a}^{\nu}=0
$$

e.g. $\tilde{p}_{a}$ is orthogonal to the spin correlation tensor. We start with the integration of $V_{k}^{q_{a} g_{i}}$. We obtain

$$
\begin{aligned}
\mathcal{V}_{k}^{q_{a} g_{i}}= & \int d \phi_{\text {dipole }} \frac{1}{2 p_{i} p_{a}} V_{k}^{q_{a} g_{i}}=\frac{\alpha_{s}}{2 \pi} \frac{1}{\Gamma(1-\varepsilon)}\left(\frac{4 \pi \mu^{2}}{m_{k}^{2}-P^{2}}\right)^{\varepsilon} \mathcal{V}^{q q}\left(x, x_{0}, \varepsilon\right), \\
\mathcal{V}^{q q}\left(x, x_{0}, \varepsilon\right) & \\
= & x^{\varepsilon}(1-x)^{-2 \varepsilon}\left(1-x_{0} x\right)^{\varepsilon} \int_{0}^{1} d w w^{-\varepsilon-1}(1-w)^{-\varepsilon} \\
& C_{F} \delta_{s s^{\prime}}\left[\frac{2}{1-x+u}-(1+x)-\rho \varepsilon(1-x)\right] \\
= & C_{F} \delta_{s s^{\prime}} x^{\varepsilon}(1-x)^{-2 \varepsilon}\left(1-x_{0} x\right)^{\varepsilon} \frac{\Gamma(-\varepsilon) \Gamma(1-\varepsilon)}{\Gamma(1-2 \varepsilon)} \\
& \cdot\left(\frac{2}{1-x}{ }_{2} F_{1}\left(1,-\varepsilon ; 1-2 \varepsilon ; \frac{-1}{1-x_{0} x}\right)-(1+x)-\rho \varepsilon(1-x)\right) .
\end{aligned}
$$


$\mathcal{V}^{q q}$ is a distribution in $x$. In order to obtain expressions which are integrable at $x=1$ we rewrite

$$
(1-x)^{-2 \varepsilon-1}=\left.(1-x)^{-2 \varepsilon-1}\right|_{+}+\delta(1-x) \int_{0}^{1} d y(1-y)^{-2 \varepsilon-1}
$$

where

$$
\left.(1-x)^{-2 \varepsilon-1}\right|_{+}=\left.\frac{1}{1-x}\right|_{+}-\left.2 \varepsilon \frac{\ln (1-x)}{1-x}\right|_{+}+O\left(\varepsilon^{2}\right)
$$

In order to expand the hypergeometric function we use the analytical continuation formula [73]

$$
\begin{aligned}
&{ }_{2} F_{1}(a, b ; c ;-x)=(x)^{-a} \frac{\Gamma(c) \Gamma(b-a)}{\Gamma(b) \Gamma(c-a)}{ }_{2} F_{1}\left(a, 1+a-c ; 1+a-b ;-\frac{1}{x}\right) \\
&+(x)^{-b} \frac{\Gamma(c) \Gamma(a-b)}{\Gamma(a) \Gamma(c-b)}{ }_{2} F_{1}\left(b, 1+b-c ; 1+b-a ;-\frac{1}{x}\right), \\
&|\arg x|<\pi .
\end{aligned}
$$

We obtain

$$
\begin{aligned}
\mathcal{V}^{q q}\left(x, x_{0}, \varepsilon\right) & C_{F} \delta_{s s^{\prime}}\left[-\frac{1}{\varepsilon}\left(\left.\frac{2}{1-x}\right|_{+}-(1+x)\right)+\rho(1-x)\right. \\
& +\delta(1-x)\left(\frac{1}{\varepsilon^{2}}+\frac{1}{\varepsilon} \ln \left(2-x_{0}\right)+\frac{\pi^{2}}{6}+2 \ln \left(1-x_{0}\right) \ln \left(2-x_{0}\right)+2 \operatorname{Li}_{2}\left(x_{0}-1\right)\right. \\
& \left.-\frac{1}{2} \ln ^{2}\left(2-x_{0}\right)\right)-2\left(\ln x+\ln \left(2-x_{0} x\right)\right)\left(\left.\frac{1}{1-x}\right|_{+}\right)+\left.4 \frac{\ln (1-x)}{1-x}\right|_{+} \\
& \left.+(1+x)\left(\ln x-2 \ln (1-x)+\ln \left(1-x_{0} x\right)\right)\right]+O(\varepsilon)
\end{aligned}
$$

For the integration of the other dipole terms we proceed in a similar way and we obtain

$$
\begin{aligned}
\mathcal{V}_{k}^{g_{a} \bar{q}_{i}} & =\int d \phi_{\text {dipole }} \frac{1}{2 p_{i} p_{a}} \frac{n_{s}(q)}{n_{s}(g)} V_{k}^{g_{a} \bar{q}_{i}}=\frac{\alpha_{s}}{2 \pi} \frac{1}{\Gamma(1-\varepsilon)}\left(\frac{4 \pi \mu^{2}}{m_{k}^{2}-P^{2}}\right)^{\varepsilon} \mathcal{V}^{g q}\left(x, x_{0}, \varepsilon\right), \\
\mathcal{V}^{g q}\left(x, x_{0}, \varepsilon\right) & \\
& =x^{\varepsilon}(1-x)^{-2 \varepsilon}\left(1-x_{0} x\right)^{\varepsilon} \int_{0}^{1} d w w^{-\varepsilon-1}(1-w)^{-\varepsilon} T_{R} \delta_{s s^{\prime}} \frac{1-\rho \varepsilon-2 x(1-x)}{1-\rho \varepsilon} \\
& =T_{R} \delta_{s s^{\prime}} x^{\varepsilon}(1-x)^{-2 \varepsilon}\left(1-x_{0} x\right)^{\varepsilon} \frac{\Gamma(-\varepsilon) \Gamma(1-\varepsilon)}{\Gamma(1-2 \varepsilon)} \frac{1-\rho \varepsilon-2 x(1-x)}{1-\rho \varepsilon}
\end{aligned}
$$




$$
\begin{aligned}
= & T_{R} \delta_{s s^{\prime}}\left[-\frac{1}{\varepsilon}\left(x^{2}+(1-x)^{2}\right)+2 \rho x(1-x)\right. \\
& \left.-\left(x^{2}+(1-x)^{2}\right)\left(\ln x-2 \ln (1-x)+\ln \left(1-x_{0} x\right)\right)\right] \\
& +O(\varepsilon),
\end{aligned}
$$

$$
\begin{aligned}
\mathcal{V}_{k}^{q_{a} q_{i}}= & \int d \phi_{\text {dipole }} \frac{1}{2 p_{i} p_{a}} \frac{n_{s}(g)}{n_{s}(q)} V_{k}^{q_{a} q_{i}}=\frac{\alpha_{s}}{2 \pi} \frac{1}{\Gamma(1-\varepsilon)}\left(\frac{4 \pi \mu^{2}}{m_{k}^{2}-P^{2}}\right)^{\varepsilon} \mathcal{V}^{q g}\left(x, x_{0}, \varepsilon\right) \\
\mathcal{V}^{q g}\left(x, x_{0}, \varepsilon\right) & \\
= & x^{\varepsilon}(1-x)^{-2 \varepsilon}\left(1-x_{0} x\right)^{\varepsilon} \int_{0}^{1} d w w^{-\varepsilon-1}(1-w)^{-\varepsilon} \\
& C_{F}\left[-g^{\mu \nu} x+4 \frac{1-x}{x} \frac{r(1-r)}{2 p_{i} q} S^{\mu \nu}\right](1-\rho \varepsilon) \\
= & C_{F}\left(-g^{\mu \nu}\right) \frac{\Gamma(-\varepsilon) \Gamma(1-\varepsilon)}{\Gamma(1-2 \varepsilon)} x^{\varepsilon}(1-x)^{-2 \varepsilon}\left(1-x_{0} x\right)^{\varepsilon} \\
& \left(x+\frac{2}{1-\rho \varepsilon} \frac{1-x}{x}\right)(1-\rho \varepsilon) \\
& + \text { gauge terms } \\
= & C_{F}\left(-g^{\mu \nu}\right)\left[-\frac{1}{\varepsilon}\left(x+2 \frac{1-x}{x}\right)+\rho x-\left(x+2 \frac{1-x}{x}\right)(\ln x-2 \ln (1-x)\right. \\
& \left.\left.+\ln \left(1-x_{0} x\right)\right)\right] \\
& + \text { gauge terms }+O(\varepsilon),
\end{aligned}
$$

$$
\begin{aligned}
\mathcal{V}_{k}^{g_{a} g_{i}}= & \int d \phi_{\text {dipole }} \frac{1}{2 p_{i} p_{a}} V_{k}^{g_{a} g_{i}}=\frac{\alpha_{s}}{2 \pi} \frac{1}{\Gamma(1-\varepsilon)}\left(\frac{4 \pi \mu^{2}}{m_{k}^{2}-P^{2}}\right)^{\varepsilon} \mathcal{V}^{g g}\left(x, x_{0}, \varepsilon\right), \\
\mathcal{V}^{g g}\left(x, x_{0}, \varepsilon\right) & \\
= & x^{\varepsilon}(1-x)^{-2 \varepsilon}\left(1-x_{0} x\right)^{\varepsilon} \int_{0}^{1} d w w^{-\varepsilon-1}(1-w)^{-\varepsilon} \\
& C_{A}\left[-g^{\mu \nu}\left(\frac{1}{1-x+u}-1+x-x^{2}\right)+2(1-\rho \varepsilon) \frac{1-x}{x} \frac{r(1-r)}{2 p_{i} q} S^{\mu \nu}\right] \\
= & 2 C_{A}\left(-g^{\mu \nu}\right) x^{\varepsilon}(1-x)^{-2 \varepsilon}\left(1-x_{0} x\right)^{\varepsilon} \frac{\Gamma(-\varepsilon) \Gamma(1-\varepsilon)}{\Gamma(1-2 \varepsilon)} \\
& \cdot\left[\frac{1}{1-x}{ }^{2} F_{1}\left(1,-\varepsilon ; 1-2 \varepsilon ; \frac{-1}{1-x_{0} x}\right)-1+x-x^{2}+\frac{1-x}{x}\right] \\
& + \text { gauge terms } \\
= & 2 C_{A}\left(-g^{\mu \nu}\right)\left[-\left.\frac{1}{\varepsilon} \frac{1}{1-x}\right|_{+}-\left(\ln x+\ln \left(2-x_{0} x\right)\right)\left(\left.\frac{1}{1-x}\right|_{+}\right)+\left.2 \frac{\ln (1-x)}{1-x}\right|_{+}\right.
\end{aligned}
$$




$$
\begin{aligned}
& +\left(\frac{1}{\varepsilon}+\ln x-2 \ln (1-x)+\ln \left(1-x_{0} x\right)\right)\left(1-x+x^{2}-\frac{1-x}{x}\right) \\
& +\delta(1-x)\left(\frac{1}{2 \varepsilon^{2}}+\frac{\pi^{2}}{12}+\frac{1}{2 \varepsilon} \ln \left(2-x_{0}\right)+\ln \left(1-x_{0}\right) \ln \left(2-x_{0}\right)+\operatorname{Li}_{2}\left(x_{0}-1\right)\right. \\
& \left.\left.-\frac{1}{4} \ln ^{2}\left(2-x_{0}\right)\right)\right]+ \text { gauge terms }+O(\varepsilon)
\end{aligned}
$$

Here $n_{s}(q)=2$ denotes the polarizations of a fermion and $n_{s}(g)=2(1-\rho \varepsilon)$ denotes the polarizations of a gluon. Note that the dependence on the momentum $q$ in $\mathcal{V}_{k}^{q_{a} q_{i}}$ and $\mathcal{V}_{k}^{g_{a} g_{i}}$ dropped out after integration.

\subsubsection{Massive final emitter, massless initial spectator}

We now consider the case of a massive emitter (particle $k$ ) in the final state and a massless spectator (particle $a$ ) in the initial state. It is sufficient to consider the case where a heavy quark (or antiquark) emits a gluon. The variables $z$ and $x$ of Catani and Seymour are given by

$$
z=\frac{2 p_{k} p_{a}}{2 p_{i} p_{a}+2 p_{k} p_{a}}, \quad x=\frac{2 p_{i} p_{a}+2 p_{k} p_{a}-2 p_{i} p_{k}}{2 p_{i} p_{a}+2 p_{k} p_{a}} .
$$

The variables $z$ and $w$ are related by

$$
z=1-\frac{1-x}{1-x_{0} x} w, \quad x_{0}=1-\frac{m_{k}^{2}}{m_{k}^{2}-\left(p_{k}+p_{i}-p_{a}\right)^{2}} .
$$

Since there is no collinear singularity, we just have to match the part of the soft singularity which corresponds to this dipole factor:

$$
\left\langle s\left|V_{q_{k} g_{i}}^{a}\right| s^{\prime}\right\rangle=8 \pi \mu^{2 \varepsilon} \alpha_{s} C_{F} \delta_{s s^{\prime}}\left[\frac{2}{1-z+(1-x)}-2\left(1-x_{0}\right) \frac{x^{2}}{1-x}\right] .
$$

We use the same mapping for the momenta as in the previous section:

$$
\begin{aligned}
& \tilde{p}_{a}=x p_{a} \\
& \tilde{p}_{k}=p_{k}+p_{i}-(1-x) p_{a} .
\end{aligned}
$$

This mapping fulfills the asymptotic behavior

$$
\lim _{2 p_{k} p_{i} \rightarrow 0} x=1, \quad \lim _{2 p_{k} p_{i} \rightarrow 0} \tilde{p}_{k}=p_{k}+p_{i}, \quad \lim _{2 p_{k} p_{i} \rightarrow 0} \tilde{p}_{a}=p_{a} .
$$

Integration yields

$$
\mathcal{V}_{q_{k} g_{i}}^{a}=\int d \phi_{\text {dipole }} \frac{1}{2 p_{i} p_{k}} V_{q_{k} g_{i}}^{a}=\frac{\alpha_{s}}{2 \pi} \frac{1}{\Gamma(1-\varepsilon)}\left(\frac{4 \pi \mu^{2}}{m_{k}^{2}-P^{2}}\right)^{\varepsilon} \mathcal{V}_{Q g}\left(x, x_{0}, \varepsilon\right)
$$




$$
\begin{aligned}
& \mathcal{V}_{Q g}\left(x, x_{0}, \varepsilon\right) \\
& =x^{\varepsilon}(1-x)^{-2 \varepsilon}\left(1-x_{0} x\right)^{\varepsilon-1} \int_{0}^{1} d w w^{-\varepsilon}(1-w)^{-\varepsilon} \\
& C_{F}\left[\frac{2}{1-z+(1-x)}-2\left(1-x_{0}\right) \frac{x^{2}}{1-x}\right] \\
& =C_{F} \delta_{s s^{\prime}} x^{\varepsilon}(1-x)^{-2 \varepsilon}\left(1-x_{0} x\right)^{\varepsilon-1} \frac{\Gamma(1-\varepsilon)^{2}}{\Gamma(2-2 \varepsilon)} \\
& \cdot\left(\frac{2}{1-x}{ }_{2} F_{1}\left(1,1-\varepsilon ; 2-2 \varepsilon ; \frac{-1}{1-x_{0} x}\right)-2\left(1-x_{0}\right) \frac{x^{2}}{1-x}\right) \\
& =C_{F} \delta_{s s^{\prime}}\left[\delta ( 1 - x ) \left(\frac{1}{\varepsilon}\left(1+\ln \left(1-x_{0}\right)-\ln \left(2-x_{0}\right)\right)+2-\frac{\pi^{2}}{3}+\ln \left(1-x_{0}\right)\right.\right. \\
& \left.+\frac{1}{2} \ln ^{2}\left(1-x_{0}\right)+\frac{1}{2} \ln ^{2}\left(2-x_{0}\right)-2 \ln \left(1-x_{0}\right) \ln \left(2-x_{0}\right)-2 \operatorname{Li}_{2}\left(x_{0}-1\right)\right) \\
& \left.+2\left(\ln \left(2-x_{0} x\right)-\ln \left(1-x_{0} x\right)-\frac{\left(1-x_{0}\right) x^{2}}{1-x_{0} x}\right)\left(\left.\frac{1}{1-x}\right|_{+}\right)\right] \\
& +O(\varepsilon) \text {. }
\end{aligned}
$$

\subsection{Conclusions}

In this chapter we have extended the dipole formalism to processes involving heavy fermions. We gave the explicit subtraction terms, together with a mapping of the momenta from the $(n+1)$-parton configuration to the $n$-parton configuration. We evaluated the integrals of the subtraction terms over the dipole phase space to order $O\left(\varepsilon^{0}\right)$. These ingredients are sufficient to set up numerical NLO programs based on the dipole formalism for processes involving heavy fermions. This we will now do in the next chapter for single-top production. 


\section{Chapter 4}

\section{Next to Leading Order}

\subsection{Introduction}

In this chapter we present and discuss new calculations of single-top-quark production at Next to Leading Order (NLO) in QCD. Earlier calculations of the NLO single-topquark production cross sections exist in the literature. The first calculation [74] was for the double differential cross section and used small masses for the gluons and quarks to regularize infrared and collinear divergences. Mass factorization was performed in the Deep Inelastic Scattering (DIS) scheme. Subsequent NLO calculations for the $s$-channel $[75,76]$ and $t$-channel $[77,78]$ modes used dimensional regularization and expressed the semi-inclusive cross section in terms of the Modified Minimal Subtraction $(\overline{\mathrm{MS}})$ factorization scheme. The value of our work is that the results are fully differential (meaning experimental cuts and jet finders can be applied), the results contain spin information, and the results use standard methods and schemes. The calculational methods used are an instructive step toward the computation of NLO corrections to the $t \bar{b} j$ production channel that should also be considered when studying single-topquark production.

The NLO cross section receives contributions from virtual corrections and real emission diagrams. Taken separately, both parts are divergent and therefore cannot be evaluated in a straightforward way numerically on a computer. Only the sum of the virtual corrections and the real emission contributions is finite after mass factorization. Writing a general-purpose NLO Monte Carlo based program therefore requires the analytic cancellation of singularities before any numerical integration can be performed.

The two main general methods to handle the cancellation of singularities without loss of information are the phase space slicing [46, 48, 79, 51, 56, 57, 58, 52] and the subtraction $[49,50,55,60,59,45,5,80]$ methods. In this chapter we implement the phase space slicing method of one [58] and two [52] cutoffs, and the subtractionbased dipole formalism of Ref. [5]. We find that the results of all methods agree. The 
dipole calculation uses helicity amplitudes and therefore contains the complete spin correlations of the participating partons.

We organize this chapter as follows. In the next section we present an overview of the amplitudes entering the calculation. Section 4.3 discusses the framework of the phase space slicing method and presents the attendant analytic results. Section 4.4 gives information relevant to the dipole calculation and all relevant helicity amplitudes. This section also contains a detailed discussion of scheme independence for different ways of handling $\gamma_{5}$. The analytic results of Sections 4.3 and 4.4 are presented using different, but self-consistent, notations appropriate to their methods of handling of infrared divergences. Brief numerical results are presented in Section 4.5, along with a comparison of the methods. We conclude in Section 4.6.

\subsection{Overview}

The lowest-order Feynman diagrams are shown in Figure 4.1. They are commonly distinguished by the sign of the $W$ boson momentum squared. The $t$-channel flavor excitation process

$$
u+b \rightarrow t+d
$$

occurs via the exchange of a virtual space-like $W$-boson, and the Drell-Yan-like $s$ channel process

$$
u+\bar{d} \rightarrow t+\bar{b}
$$

occurs via a virtual time-like $W$-boson. In reaction (4.1) it is understood that we may replace the $(u, d)$-quark pair by $(\bar{d}, \bar{u}),(c, s)$ and $(\bar{s}, \bar{c})$. In reaction (4.2) we may replace the $(u, \bar{d})$-pair by $(c, \bar{s})$. In addition, CKM suppressed combinations are included at all vertices.

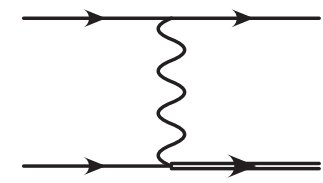

(a)

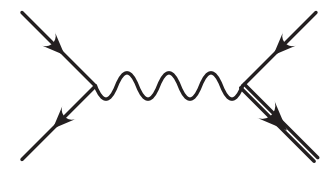

(b)

Figure 4.1: The leading order Feynman diagrams for reactions given in (a) Eq. (4.1) and (b) Eq. (4.2). The top quark line is doubled.

For each channel the fully differential spin-averaged Born cross section can be written as

$$
d \sigma_{p}^{(0)}=\frac{1}{2 s} \bar{\sum}\left|\mathcal{M}_{p, \text { Born }}\right|^{2} d \mathrm{PS}_{2},
$$


where $s$ is the partonic center-of-momentum energy squared, and we use $p=s, t$ to denote the channel. The two body phase space is given by

$$
d \mathrm{PS}_{2}=\frac{1}{(2 \pi)^{2}} \frac{d^{3} p_{1}}{2 E_{1}} \frac{d^{3} p_{2}}{2 E_{2}} \delta^{(4)}\left(q-p_{1}-p_{2}\right) .
$$

The $t$-channel Born matrix element squared summed (averaged) over final (initial) state spin and color states is

$$
\bar{\sum}\left|\mathcal{M}_{t, \text { Born }}\right|^{2}=\frac{1}{4} g^{4}\left|V_{u d}\right|^{2}\left|V_{t b}\right|^{2} s\left(s-m_{t}^{2}\right)\left|\frac{1}{t-M_{W}^{2}}\right|^{2} .
$$

Here, $s=\left(p_{u}+p_{b}\right)^{2}$ and $t=\left(p_{u}-p_{d}\right)^{2}$, the partonic reaction sub-energy squared and the square of the momentum transfer across the $W$, respectively. The CKM matrix elements $\left|V_{i j}\right|$ may be changed for the given particles, and $m_{t}$ is the top-quark mass. The result for the $s$-channel is obtained by interchanging $s$ and $t$, and letting $s=\left(p_{u}+p_{\bar{d}}\right)^{2}$ and $t=\left(p_{u}-p_{\bar{b}}\right)^{2}$. In Section 4.4 we discuss the cross section without the sum over spins.
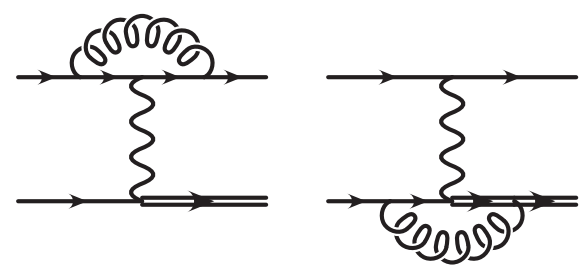

(a)

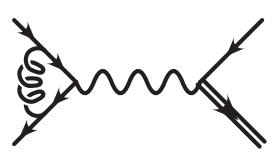

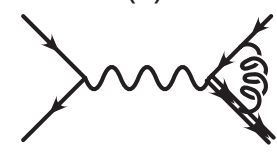

(b)
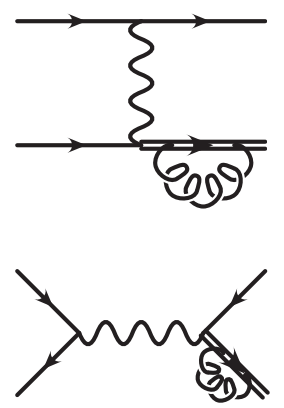

Figure 4.2: The one-loop virtual corrections to the (a) t-channel and (b) s-channel amplitudes.

At next to leading order we must include the virtual QCD corrections to Eqs. (4.1) and (4.2), shown in Figure 4.2. To obtain the real emission contributions one must attach a gluon in all possible ways to the diagrams in Figure 4.1. The resulting crossed diagrams may be written as

$$
\begin{aligned}
& u+\bar{d} \rightarrow t+\bar{b}+g, \\
& u+b \rightarrow t+d+g \\
& \bar{d}+b \rightarrow t+\bar{u}+g, \\
& u+g \rightarrow t+\bar{b}+d
\end{aligned}
$$




$$
\begin{aligned}
\bar{d}+g & \rightarrow t+\bar{b}+\bar{u} \\
g+b & \rightarrow t+d+\bar{u} .
\end{aligned}
$$

These crossings may belong either to the $s$ or $t$ channel, and their assignment is governed by the collinear singularity structure. It is useful to distinguish whether the gluon attaches to the fermion current containing the heavy-quark line $(h)$ in Figure 4.1 or to the current containing only the light-quark lines $(l)$. Note that the contributions from $h$ and $l$ radiation do not interfere in the cross section due to color conservation [75, 77].

The diagrams in Eq. (4.6) belong fully to the $s$-channel, and diagrams in Eq. (4.7) belong to the $t$-channel. For crossing in Eq. (4.8) let us consider the first process $u+g \rightarrow t+\bar{b}+d$. In the heavy-quark line the gluon can split into a collinear $b \bar{b}$-pair with reduced process $u+b \rightarrow t+d$, so that this crossing belongs to the $t$-channel. In the light-quark line the gluon can split into a collinear $d \bar{d}$-pair, with reduced process $u+\bar{d} \rightarrow t+\bar{b}$, so that this crossing belongs to the $s$-channel. The classification of the second process in Eq. (4.8) is similar, with the role of the $u$ - and $d$-type quarks exchanged. Finally, in Eq. (4.9), the gluon connected to the light-quark line can split either into a collinear $u \bar{u}$-pair or into a collinear $d \bar{d}$-pair. In both cases the process reduces to the $t$-channel process $u+b \rightarrow t+d$ or $\bar{d}+b \rightarrow t+\bar{u}$. Crossing of Eq. (4.9) to the heavy-quark line is not included in our calculation as it involves on-shell $W$-decay into light fermions, and is classified as $W$-top associated production. The cross section for this process is estimated to be negligibly small for the Tevatron [15, 16, 18, 81, 82].

In what follows we consider only the production of a single top quark, but the relevant expressions for the production of an antitop quark may easily be obtained by charge conjugation. Our calculational framework is further specified as follows: we set the bottom quark mass to zero, so that we work with 5 massless flavors in the parton distribution functions. It is important to use a consistent set of 5-flavor parton distribution functions, in which the 5-flavor set has been computed from a lower flavor number set via NLO matching conditions [83] that preserve the momentum sum rule. To handle divergences occurring at intermediate stages of the calculation we use dimensional regularization $(D=4-2 \epsilon)$. Details describing renormalization of the vertices that contain $\gamma_{5}$ are given in Section 4.4.2. Collinear divergences are subtracted in the standard $\overline{\mathrm{MS}}$ scheme. We use the Feynman gauge for the gluon propagator.

\subsection{Phase space slicing}

In the phase space slicing method a subregion of phase space containing soft and collinear singularities is defined. If the subregion is small enough, one may make simplifying kinematic approximations in the phase space integrals, so that they can be performed analytically. To define the size and shape of this subregion one introduces 
one or two theoretical cutoff parameters (for common or separate treatment of collinear and soft contributions, respectively). The final cross section should not depend on the choice made for the partitioning. Below we discuss the application of these methods to single-top-quark production.

\subsubsection{Phase space slicing with two cutoffs $\left(\delta_{s}, \delta_{c}\right)$}

A detailed description of the phase space slicing method with two cutoffs has recently been given in Ref. [52]. In this section we present the results needed for single-top-quark production. We follow closely the notation of Ref. [52].

In the two cutoff method, phase space is divided into a hard $(\mathrm{H})$ and $\operatorname{soft}(\mathrm{S})$ region. The contribution from the latter region is computed by applying the eikonal approximation to the radiative processes in Eqs. (4.6-4.9). The soft region of phase space is defined by a condition on the energy of the soft gluon in the partonic centerof-momentum frame:

$$
0 \leq E_{g} \leq \delta_{s} \frac{\sqrt{s}}{2}
$$

The hard region is then defined by $E_{g}>\delta_{s} \sqrt{s} / 2$. After performing the $D$-dimensional angular integrations of the gluon in the region defined in Eq. (4.10), the soft contribution is found to be

$$
d \sigma_{p}^{(S)}=d \sigma_{p}^{(0)}\left[\frac{\alpha_{s}}{2 \pi} \frac{\Gamma(1-\epsilon)}{\Gamma(1-2 \epsilon)}\left(\frac{4 \pi \mu^{2}}{s}\right)^{\epsilon}\right]\left(\frac{A_{2}^{p}}{\epsilon^{2}}+\frac{A_{1}^{p}}{\epsilon}+A_{0}^{p}\right),
$$

with $p=s, t$ labeling the channel. For the $s$-channel

$$
\begin{aligned}
A_{2}^{s} & =3 C_{F} \\
A_{1}^{s} & =C_{F}\left[1-6 \ln \delta_{s}-\ln \left(\frac{s}{m^{2}}\right)\right] \\
A_{0}^{s} & =C_{F}\left[6 \ln ^{2} \delta_{s}-2 \ln \delta_{s}+2 \ln \delta_{s} \ln \left(\frac{s}{m^{2}}\right)\right. \\
& \left.+\frac{s+m^{2}}{s-m^{2}} \ln \left(\frac{s}{m^{2}}\right)-2 \operatorname{Li}_{2}(\beta)-\frac{1}{2} \ln ^{2}\left(\frac{s}{m^{2}}\right)\right],
\end{aligned}
$$

and for the $t$-channel

$$
\begin{aligned}
& A_{2}^{t}=3 C_{F} \\
& A_{1}^{t}=C_{F}\left[1-6 \ln \delta_{s}-2 \ln \left(\frac{-t}{s \beta}\right)-\ln \left(\frac{\left(m^{2}-t\right)^{2}}{m^{2} s}\right)\right] \\
& A_{0}^{t}=C_{F}\left[6 \ln ^{2} \delta_{s}-2 \ln \delta_{s}+4 \ln \delta_{s} \ln \left(\frac{-t}{s \beta}\right)\right.
\end{aligned}
$$




$$
\begin{aligned}
& +2 \ln \delta_{s} \ln \left(\frac{\left(m^{2}-t\right)^{2}}{m^{2} s}\right)+\frac{s+m^{2}}{s-m^{2}} \ln \left(\frac{s}{m^{2}}\right)+\ln ^{2}\left(\frac{-t}{s \beta}\right)+2 \operatorname{Li}_{2}\left(1+\frac{t}{s \beta}\right) \\
& \left.-\frac{1}{2} \ln ^{2}\left(\frac{s}{m^{2}}\right)+\ln ^{2}\left(\frac{m^{2}}{m^{2}-t}\right)+2 \operatorname{Li}_{2}\left(\frac{t}{m^{2}}\right)-2 \operatorname{Li}_{2}\left(\frac{u}{s+u}\right)\right],
\end{aligned}
$$

where the top-quark mass is denoted as $m$, and $\beta=1-m^{2} / s$.

The hard region is further divided into hard collinear (HC) and hard-noncollinear $(\mathrm{H} \overline{\mathrm{C}})$ regions. The latter is computed in 4 dimensions, integrating numerically over the $\mathrm{H} \overline{\mathrm{C}}$ phase space using standard Monte Carlo methods. The HC contributions arise from integration over those regions of phase space where any invariant, $s_{i j}=\left(p_{i}+p_{j}\right)^{2}$ or $t_{i j}=\left(p_{i}-p_{j}\right)^{2}$, appearing in the denominator becomes smaller in magnitude than $\delta_{c} s$. The singular regions are distinguished by whether they come from initial or final state radiation. The latter are given by

$$
d \sigma_{p}^{(H C, F S)}=d \sigma_{p}^{(0)}\left[\frac{\alpha_{s}}{2 \pi} \frac{\Gamma(1-\epsilon)}{\Gamma(1-2 \epsilon)}\left(\frac{4 \pi \mu^{2}}{s}\right)^{\epsilon}\right]\left(\frac{A_{1}}{\epsilon}+A_{0}\right)
$$

with

$$
\begin{aligned}
A_{1}= & C_{F}\left(2 \ln \delta_{s}+\frac{3}{2}-2 \ln \beta\right) \\
A_{0}= & C_{F}\left[\frac{7}{2}-\frac{\pi^{2}}{3}-\ln ^{2} \delta_{s}-\ln ^{2} \beta+2 \ln \delta_{s} \ln \beta\right. \\
& \left.-\ln \delta_{c}\left(2 \ln \delta_{s}+\frac{3}{2}-2 \ln \beta\right)\right],
\end{aligned}
$$

for both channels (all massless partons in the Born cross sections are fermions).

The collinear contributions from the initial state are given by the sum of two contributions. The first is the finite remainder after mass factorization. The second results from a mismatch in integration limits when subtracting the mass factorization counterterm.

$$
d \sigma_{p, C}^{i j \rightarrow F S}=d \sigma_{p}^{(0)}\left[\frac{\alpha_{s}}{2 \pi} \frac{\Gamma(1-\epsilon)}{\Gamma(1-2 \epsilon)}\left(\frac{4 \pi \mu^{2}}{s}\right)^{\epsilon}\right]\left[\tilde{f}_{j}^{H}\left(z, \mu_{F}\right)+\left(\frac{A_{1}^{s c}}{\epsilon}+A_{0}^{s c}\right) f_{j}^{H}\left(z, \mu_{F}\right)\right] .
$$

The modified parton distribution function $\tilde{f}$ is given in Ref. [52]. For the reaction at hand we only need consider the quark-quark initial state splitting, so

$$
\begin{aligned}
& A_{1}^{s c}=C_{F}\left(2 \ln \delta_{s}+\frac{3}{2}\right) \\
& A_{0}^{s c}=C_{F}\left(2 \ln \delta_{s}+\frac{3}{2}\right) \ln \left(\frac{s}{\mu_{F}^{2}}\right) .
\end{aligned}
$$


The virtual contribution is obtained as explained in the Sections 4.4.1 and 4.4.2. The results in the notation of this section are

$$
d \sigma_{p}^{(V)}=d \sigma_{p}^{(0)}\left[\frac{\alpha_{s}}{2 \pi} \frac{\Gamma(1-\epsilon)}{\Gamma(1-2 \epsilon)}\left(\frac{4 \pi \mu^{2}}{s}\right)^{\epsilon}\right]\left(\frac{A_{2}^{V}}{\epsilon^{2}}+\frac{A_{1}^{V}}{\epsilon}+A_{0}^{V}\right)+\left(\frac{\alpha_{s}}{2 \pi}\right) d \tilde{\sigma}_{p}^{(V)},
$$

where

$$
\begin{aligned}
A_{2}^{V} & =C_{F}\{[-2]-[1]\} \\
A_{1}^{V} & =C_{F}\left\{\left[-3-2 \ln \left(\frac{s}{-q^{2}}\right)\right]+\left[-\frac{5}{2}-2 \ln (1-\lambda)-\ln \left(\frac{s}{m^{2}}\right)\right]\right\} \\
A_{0}^{V} & =C_{F}\left\{\left[-\ln ^{2}\left(\frac{s}{-q^{2}}\right)-3 \ln \left(\frac{s}{-q^{2}}\right)-8-\frac{\pi^{2}}{3}\right]\right. \\
& +\left[-\frac{1}{2} \ln ^{2}\left(\frac{s}{m^{2}}\right)-\frac{5}{2} \ln \left(\frac{s}{m^{2}}\right)-2 \ln (1-\lambda) \ln \left(\frac{s}{m^{2}}\right)-6\right. \\
& \left.\left.-\frac{1}{\lambda} \ln (1-\lambda)-\ln ^{2}(1-\lambda)-2 \ln (1-\lambda)+2 \operatorname{Li}_{2}(\lambda)-\frac{\pi^{2}}{3}\right]\right\} .
\end{aligned}
$$

In the above we have defined $\lambda \equiv q^{2} /\left(q^{2}-m^{2}\right)$. Further, the separate terms in the square brackets originate from the massless-massless or the massive-massless vertex corrections. For the $t$-channel one sets $q^{2}=t$, while for the $s$-channel one sets $q^{2}=s$.

$$
d \tilde{\sigma}_{t}^{(V)}=\frac{1}{2 s} \frac{1}{4} g^{4}\left|V_{u d}\right|^{2}\left|V_{t b}\right|^{2} C_{F} \frac{m^{2} s u}{t} \ln \left(\frac{m^{2}}{m^{2}-t}\right)\left(\frac{1}{t-M_{W}^{2}}\right)^{2} d \Gamma_{2},
$$

is the $t$-channel finite piece in the virtual contribution that is not proportional to the Born cross section. It results from the interference of the renormalized massive-massless vertex with the Born amplitude. The $s$-channel version may be obtained by crossing.

At this point one can see that the two body weight is finite: $A_{2}^{p}+A_{2}^{V}=0$ and $A_{1}^{p}+A_{1}^{V}+A_{1}+2 A_{1}^{s c}=0$. The factor of two occurs since there are two quark legs, either of which can emit a gluon. The final finite two-body cross section is given by the sum of the residual $\tilde{f}$ terms from both the quark-quark and quark-gluon initiated processes and the finite two-body weights. The result, summed over parton flavors is

$$
\begin{aligned}
\sigma^{(2)}= & \left(\frac{\alpha_{s}}{2 \pi}\right) \sum_{a, b} \int d x_{1} d x_{2} \\
& \left\{f_{a}^{H_{1}}\left(x_{1}, \mu_{F}\right) f_{b}^{H_{2}}\left(x_{2}, \mu_{F}\right)\left[d \sigma_{p}^{(0)}\left(A_{0}^{p}+A_{0}^{V}+A_{0}+2 A_{0}^{s c}\right)+d \tilde{\sigma}_{p}^{(V)}\right]\right. \\
& \left.+d \sigma_{p}^{(0)}\left[f_{a}^{H_{1}}\left(x_{1}, \mu_{F}\right) \tilde{f}_{b}^{H_{2}}\left(x_{2}, \mu_{F}\right)+\tilde{f}_{a}^{H_{1}}\left(x_{1}, \mu_{F}\right) f_{b}^{H_{2}}\left(x_{2}, \mu_{F}\right)\right]+\left(x_{1} \leftrightarrow x_{2}\right)\right\} .
\end{aligned}
$$

The three-body contribution is given by

$$
\sigma^{(3)}=\sum_{a, b} \int d x_{1} d x_{2} \frac{1}{2 s} \int_{H \bar{C}} \bar{\sum}\left|M_{3}^{(a b)}\right|^{2} d \Gamma_{3},
$$


with

$$
\bar{\sum}\left|M_{3}^{(a b)}\right|^{2}=-\pi g^{4}\left|V_{u d}\right|^{2}\left|V_{t b}\right|^{2} \Psi_{i}, i=1-3 .
$$

The $\Psi_{i}$ contain the luminosity and Dirac algebra of Eqs. (4.7-4.9). We choose initial-state momenta as incoming, and label the momenta for the $t$-channel as

$$
\begin{aligned}
& \Psi_{1}: u\left(p_{1}\right) b\left(p_{2}\right) \rightarrow d\left(p_{3}\right) t\left(p_{4}\right) g\left(p_{5}\right), \\
& \Psi_{2}: u\left(p_{1}\right) g\left(p_{2}\right) \rightarrow d\left(p_{3}\right) t\left(p_{4}\right) \bar{b}\left(p_{5}\right), \\
& \Psi_{3}: g\left(p_{1}\right) b\left(p_{2}\right) \rightarrow d\left(p_{3}\right) t\left(p_{4}\right) \bar{u}\left(p_{5}\right) .
\end{aligned}
$$

The $t$-channel $\Psi_{i}$ are given by

$$
\begin{aligned}
\Psi_{1}= & 2 C_{F} F_{1}\left(\frac{s_{12}\left(t_{14}^{\prime}-s_{34}^{\prime}\right)-s_{34}^{\prime} t_{25}}{t_{15}}-\frac{s_{12}\left(s_{34}^{\prime}+s_{45}^{\prime}\right)-s_{34}^{\prime} t_{23}}{s_{35}}\right. \\
& \left.-\frac{t_{13}\left[s_{12}\left(2 s_{34}^{\prime}+s_{45}^{\prime}\right)+s_{34}^{\prime} t_{25}\right]}{t_{15} s_{35}}\right) \\
& +2 C_{F} F_{2}\left(\frac{s_{12}\left(t_{23}-s_{34}^{\prime}\right)-s_{34}^{\prime} t_{15}}{t_{25}}-\frac{s_{12}\left(s_{34}^{\prime}+s_{35}\right)\left(1-2 m_{t}^{2} / s_{45}^{\prime}\right)-s_{34}^{\prime} t_{14}^{\prime}}{s_{45}^{\prime}}\right. \\
\Psi_{2}= & F_{2}\left(\frac{s_{12} s_{34}^{\prime}+t_{15}\left(s_{34}^{\prime}-s_{35}\right)}{t_{25}}+\frac{t_{15}\left(s_{34}^{\prime}+t_{23}\right)\left(1-2 m_{t}^{2} / t_{24}^{\prime}\right)-s_{34}^{\prime} t_{14}^{\prime}}{t_{24}^{\prime}}\right. \\
& \left.+\frac{s_{45}^{\prime}\left[s_{12} s_{34}^{\prime}+t_{15}\left(t_{23}+2 s_{34}^{\prime}\right)\right]}{t_{24}^{\prime} t_{25}}\right) \\
\Psi_{3}= & F_{1}\left(\frac{s_{12} s_{34}^{\prime}+t_{25}\left(s_{34}^{\prime}-s_{45}^{\prime}\right)}{t_{15}}+\frac{t_{25}\left(s_{34}^{\prime}+t_{14}^{\prime}\right)-s_{34}^{\prime} t_{23}}{t_{13}}\right. \\
& \left.+\frac{s_{35}\left[s_{12} s_{34}^{\prime}+t_{25}\left(t_{14}^{\prime}+2 s_{34}^{\prime}\right)\right]}{t_{13} t_{15}}\right)
\end{aligned}
$$

where $C_{F}=4 / 3, s_{i j}=\left(p_{i}+p_{j}\right)^{2}, t_{i j}=\left(p_{i}-p_{j}\right)^{2}, s_{i j}^{\prime}=s_{i j}-m_{t}^{2}, t_{i j}^{\prime}=t_{i j}-m_{t}^{2}$,

$$
\begin{aligned}
& F_{1}=\frac{\alpha_{s l}}{\left(t_{24}-M_{W}^{2}\right)^{2}} L_{l}, \\
& F_{2}=\frac{\alpha_{s h}}{\left(t_{13}-M_{W}^{2}\right)^{2}} L_{h},
\end{aligned}
$$

$\alpha_{s l(h)}$ and the luminosity functions $L_{l(h)}=f_{a}^{H_{1}}\left(x_{1}, \mu_{F l(h)}\right) f_{b}^{H_{2}}\left(x_{2}, \mu_{F l(h)}\right)$ are evaluated using the scales at the light(heavy)-quark lines, respectively. All other $s$ - and $t$-channel matrix elements can be obtained by crossing. Physical predictions follow from the sum $\sigma^{(2)}+\sigma^{(3)}$, which is cutoff independent for sufficiently small cutoffs as shown below. 


\subsubsection{Phase space slicing with one cutoff $\left(s_{\min }\right)$}

The calculation using the one cutoff slicing method is similar to the one using the two cutoff slicing method, with some differences that we now address. In this method, a pair of partons with momenta $p_{i}$ and $p_{j}$ is defined to be unresolved if

$$
\left|2 p_{i} \cdot p_{j}\right|<s_{\min }
$$

with $s_{\min }$ small compared to the hard scale of the process. The condition in Eq. (4.32) can occur if either $p_{i}$ and $p_{j}$ are collinear, or if one of the two is soft. This method, combined with the use of color-decomposed amplitudes and universal crossing functions, has been developed into a general method for computing with minimal calculational effort fully differential NLO production cross sections of bosons and jets in $[56,57]$ and identified hadrons and heavy quarks in [58]. The single-top-quark production process has a relatively simple color structure, so we do not need to decompose the scattering amplitudes into color-ordered subamplitudes.

The treatment of the virtual corrections is no different from the two cutoff method. To determine the radiative corrections, all partons are first crossed to the final state, and resolved and unresolved contributions are identified according to the criterion in Eq. (4.32). The unresolved contributions, (soft and collinear) can be found in [56, 57, $58]$ expressed in $D=4-2 \epsilon$ dimensions. The soft contributions are expressed in terms of the momenta for partons in lowest order kinematics with all partons in the final state

$$
0 \rightarrow \bar{u}+d+t+\bar{b}
$$

and are given by

$$
d \sigma^{(S)}=d \sigma^{(0)}\left[\frac{\alpha_{s} C_{F}}{\pi} \frac{1}{\Gamma(1-\epsilon)}\left(\frac{4 \pi \mu^{2}}{s_{\min }}\right)^{\epsilon}\right]\left[\frac{1}{\epsilon^{2}}\left(\frac{2 p_{u} \cdot p_{d}}{s_{\min }}\right)^{\epsilon}+\mathcal{J}(m, 0)\left(\frac{2 p_{t} \cdot p_{b}}{s_{\min }}\right)^{\epsilon}\right]
$$

with $d \sigma^{(0)}$ obtained by crossing all momenta to the final state, and

$$
\begin{aligned}
& \text { 1) } 2 p_{t} \cdot p_{b} \geq m^{2}: \mathcal{J}(m, 0)=\frac{1}{\epsilon^{2}}-\frac{1}{2 \epsilon^{2}}\left(\frac{2 p_{t} \cdot p_{b}}{m^{2}}\right)^{\epsilon}+\frac{1}{2 \epsilon}\left(\frac{2 p_{t} \cdot p_{b}}{m^{2}}\right)^{\epsilon}-\frac{\pi^{2}}{12}+\frac{m^{2}}{2 p_{t} \cdot p_{b}} \\
& \text { 2) } 2 p_{t} \cdot p_{b} \leq m^{2}: \mathcal{J}(m, 0)=\left(\frac{2 p_{t} \cdot p_{b}}{m^{2}}\right)^{-\epsilon}\left(\frac{1}{2 \epsilon^{2}}+\frac{1}{2 \epsilon}-\frac{\pi^{2}}{12}+1\right) .
\end{aligned}
$$

The $s$-channel contribution is then obtained by replacing $2 p_{u} \cdot p_{d} \rightarrow s, 2 p_{t} \cdot p_{b} \rightarrow s-m^{2}$. The $t$-channel contribution is obtained by replacing $2 p_{u} \cdot p_{d} \rightarrow t, 2 p_{t} \cdot p_{b} \rightarrow t-m^{2}$, which leads to $\pi^{2}$ terms after expanding in $\epsilon$. The collinear contributions are likewise given by

$$
d \sigma^{(C)}=-d \sigma^{(0)}\left[\frac{\alpha_{s} C_{F}}{\pi} \frac{1}{\Gamma(1-\epsilon)}\left(\frac{4 \pi \mu^{2}}{s_{\min }}\right)^{\epsilon}\right] \frac{1}{\epsilon}
$$




$$
\times\left[I_{q \rightarrow q g}\left(0, \frac{s_{\min }}{2 p_{u} \cdot p_{d}}\right)+I_{\bar{q} \rightarrow q g}\left(\frac{s_{\min }}{2 p_{u} \cdot p_{d}}, 0\right)+I_{\bar{q} \rightarrow q g}\left(\frac{s_{\min }}{2 p_{t} \cdot p_{b}}, 0\right)\right]
$$

The $I$ functions are given in Refs. [56, 57]. The $s$-channel contribution is obtained by replacing $2 p_{u} \cdot p_{d} \rightarrow s, 2 p_{t} \cdot p_{b} \rightarrow s-m^{2}$. The $t$-channel contribution is obtained by replacing $2 p_{u} \cdot p_{d} \rightarrow t, 2 p_{t} \cdot p_{b} \rightarrow t-m^{2}$. The sum of these contributions is already finite. One now generates the various subprocesses of single-top-quark production by crossing pairs of partons back to the initial state. Crossing symmetry is not a property of next to leading order cross sections, but it may be implemented in the following way.

In general a NLO fully differential cross section for a process with initial hadrons $H_{1}$ and $H_{2}$ may be written as

$$
d \sigma_{H_{1} H_{2}}=\sum_{a, b} \int d x_{1} \int d x_{2} \mathcal{F}_{a}^{H_{1}}\left(x_{1}\right) \mathcal{F}_{b}^{H_{2}}\left(x_{2}\right) d \sigma_{a b}^{N L O}\left(x_{1}, x_{2}\right),
$$

where $a, b$ denote parton flavors and $x_{1}, x_{2}$ are parton momentum fractions. The function $d \sigma_{a b}^{N L O}$ is computed with all-partons-in-the-final-state matrix elements, in which partons $a$ and $b$ have simply been crossed to the initial state, i.e. in which their momenta $p_{a}$ and $p_{b}$ have been replaced by $-p_{a}$ and $-p_{b}$ (this function does include the $\pi^{2}$ terms resulting from this replacement in the one-loop virtual graphs). The functions $\mathcal{F}_{a}^{H}(x)$ are modifications of the parton distribution functions $f_{a}^{H}\left(x, \mu_{F}\right)$

$$
\mathcal{F}_{a}^{H}(x)=f_{a}^{H}\left(x, \mu_{F}\right)+\alpha_{s} C_{a}^{H}\left(x, \mu_{F}\right)+O\left(\alpha_{s}^{2}\right),
$$

where $C_{a}^{H}\left(x, \mu_{F}\right)$ are finite, universal "crossing functions" [57]. They implement the crossing property for the unresolved contributions, and are given by

$$
C_{a}^{H, \overline{\mathrm{MS}}}\left(x, \mu_{F}\right)=\frac{N_{C}}{2 \pi}\left[A_{a}^{H}\left(x, \mu_{F}\right) \ln \left(\frac{s_{\mathrm{min}}}{\mu_{F}^{2}}\right)+B_{a}^{H, \overline{\mathrm{MS}}}\left(x, \mu_{F}\right)\right] .
$$

The functions $A_{a}^{H}, B_{a}^{H}$ for the proton are given in [57]. In the unresolved contribution one may simply cross pairs of partons without further modifications.

The full NLO differential cross section can now be written as:

$$
\begin{aligned}
d \sigma_{H_{1} H_{2}}= & \sum_{a, b} \int d x_{1} d x_{2} f_{a}^{H_{1}}\left(x_{1}, \mu_{F}\right) f_{b}^{H_{2}}\left(x_{2}, \mu_{F}\right)\left[d \sigma_{a b}^{N L O}\left(x_{1}, x_{2}\right)+\alpha_{s}\left(\mu_{F}\right)\right. \\
& \left.\times\left(C_{a}^{H_{1}}\left(x_{1}, \mu_{F}\right) f_{b}^{H_{2}}\left(x_{2}, \mu_{F}\right)+f_{a}^{H_{1}}\left(x_{1}, \mu_{F}\right) C_{b}^{H_{2}}\left(x_{2}, \mu_{F}\right)\right) d \sigma_{a b}^{L O}\left(x_{1}, x_{2}\right)\right] .
\end{aligned}
$$

The unresolved contribution, now including the crossing functions, depends analytically on $s_{\min }$, but this $s_{\min }$ dependence cancels against that of the resolved contribution. The results produced with this method agree with those of the previous section. In this thesis we limit ourselves to some illustrative numerical studies, so that we only employ the dipole and two-cutoff slicing methods for numerical results. 


\subsection{Massive Dipole Subtraction calculation}

We will use the massive dipole formalism as developed in Chapter 3 for this calculation. As was explained there, we need to calculate the virtual and radiative amplitudes for the process under study, which together with the expressions for the dipoles will give us all ingredients to complete the calculation.

\subsubsection{Calculation of the amplitudes}

We have performed three different calculations of the loop amplitudes. One calculation was done using the standard approach in the 't Hooft-Veltman scheme. The second calculation involved treating the $\gamma_{5}$ as anti-commuting in $D$ dimensions, thereby retaining Ward identities for the charged current vertex. In the third one we calculated helicity amplitudes using a four-dimensional scheme. The effects of different prescriptions for $\gamma_{5}$ are discussed more extensively in Section 4.4.2. The results of the three calculations agree with each other, in the sense that they can be related to each other through process-independent finite renormalizations. In addition, we find agreement with the earlier calculations of Refs. [84, 85]. We present here the helicity amplitudes obtained with the third approach ${ }^{1}$. They are more compact and contain the complete spin information. In the standard approach one just calculates the interference between the loop amplitude and the Born term and sums over all spins.

We employ amplitudes with all partons outgoing, generating in an economical way the relevant scattering amplitudes by crossing. The lowest-order amplitude is shown in Figure 4.1

$$
A_{W b}: 0 \rightarrow t\left(p_{8}\right)+\bar{b}\left(p_{4}\right)+d\left(p_{6}\right)+\bar{u}\left(p_{7}\right),
$$

where all momenta are outgoing. We use here the notation of Ref. [4], which explains the unusual labeling of the momenta with $p_{4}, p_{6}, p_{7}$ and $p_{8}$.

Each amplitude we decompose into gauge-invariant partial amplitudes. The color decomposition of the Born amplitude is

$$
A_{W b, b o r n}=\delta_{84} \delta_{67} A_{W b, b o r n}^{[1]}
$$

It is convenient to factor out some common prefactors from the partial amplitude $A_{W b, b o r n}^{[1]}$ and to write it as follows:

$$
A_{W b, \text { born }}^{[1]}=\frac{e^{2} V_{u d}^{*} V_{t b}}{2 \sin ^{2} \theta_{W}} \cdot \frac{2 i}{s_{67}-m_{W}^{2}} \frac{B_{W b, \text { born }}^{[1]}}{\sqrt{-\langle 2-|4+6+7| 2-\rangle}} .
$$

\footnotetext{
${ }^{1}$ The spinor helicity methods that are used in this calculation are summarized in Section 2.2.
} 
Here we denote the reference momentum for the massive spinor by $q=p_{2}$. If one is only interested in the spin-summed squared amplitude, one may choose any arbitrary null vector for $p_{2}$, the choices $p_{2}=(1,0,0,1)$ or $p_{2}=p_{6}$ are examples. However, by keeping $p_{2}$ unspecified, we keep the complete spin information, and our formulas become only slightly more lengthy. The non-vanishing Born amplitudes are

$$
\begin{aligned}
& B_{W b, \text { born }}^{[1]}\left(p_{4}^{+}, p_{6}^{-}, p_{7}^{+}, p_{8}^{-}\right)=[47]\langle 6-|4+7| 2-\rangle, \\
& B_{W b, \text { born }}^{[1]}\left(p_{4}^{+}, p_{6}^{-}, p_{7}^{+}, p_{8}^{+}\right)=m\langle 26\rangle[74] .
\end{aligned}
$$

We now turn our attention to the loop amplitudes. The color decomposition of the one-loop amplitude is given by

$$
\begin{aligned}
A_{W b, \text { loop }}= & \delta_{67} T_{8 i}^{a} T_{i 4}^{a} A_{W b, \text { loop }}^{[1]}+T_{6 i}^{a} T_{i 7}^{a} \delta_{84} A_{W b, \text { loop }}^{[2]}+T_{67}^{a} T_{84}^{a} A_{W b, \text { loop }}^{[3]} \\
= & \frac{N^{2}-1}{2 N} \delta_{67} \delta_{84} A_{W b, \text { loop }}^{[1]}+\frac{N^{2}-1}{2 N} \delta_{67} \delta_{84} A_{W b, \text { loop }}^{[2]} \\
& +\frac{1}{2}\left(\delta_{64} \delta_{87}-\frac{1}{N} \delta_{67} \delta_{84}\right) A_{W b, \text { loop }}^{[3]} .
\end{aligned}
$$

Here we used the short-hand notation

$$
T_{84}^{a}=T_{i_{8} j_{4}}^{a},
$$

where $a$ is the color index of the gluon, $i_{8}$ is the color index of the quark $t\left(p_{8}\right)$ and $j_{4}$ is the color index of the quark $\bar{b}\left(p_{4}\right)$. $A_{W b \text {, loop }}^{[1]}$ corresponds to loop corrections on the $t-b$ line, $A_{W b, l o o p}^{[2]}$ to corrections on the $u$ - $d$ line, and $A_{W b, \text { loop }}^{[3]}$ to a gluon exchange between the two lines. Note that we do not have to calculate $A_{W b, l o o p}^{[3]}$ :

$$
2 \operatorname{Re}\left(\delta_{84} \delta_{67} A_{W b}^{[1]}\right)^{*} T_{67}^{a} T_{84}^{a} A_{W b, l o o p}^{[3]}=0,
$$

because the color matrices are traceless. We write

$$
\begin{aligned}
& A_{W b, \text { loop }}^{[1]}=\frac{e^{2} V_{u d}^{*} V_{t b}}{2 \sin ^{2} \theta_{W}} \frac{2 i}{s_{67}-m_{W}^{2}} \frac{B_{W b, \text { loop }}^{[1]}}{\sqrt{-\langle 2-|4+6+7| 2-\rangle}} \frac{g^{2}}{(4 \pi)^{2}} \\
& A_{W b, \text { loop }}^{[2]}=\frac{e^{2} V_{u d}^{*} V_{t b}}{2 \sin ^{2} \theta_{W}} \frac{2 i}{s_{67}-m_{W}^{2}} \frac{B_{W b, \text { loop }}^{[2]}}{\sqrt{-\langle 2-|4+6+7| 2-\rangle}} \frac{g^{2}}{(4 \pi)^{2}} .
\end{aligned}
$$

For the helicity configuration $p_{4}^{+}, p_{6}^{-}, p_{7}^{+}, p_{8}^{-}$we obtain

$$
\begin{aligned}
& B_{W b, \text { loop }}^{[1]}\left(p_{4}^{+}, p_{6}^{-}, p_{7}^{+}, p_{8}^{-}\right)= \\
& \quad 2[47]\left\{\langle 6-|4+7| 2-\rangle\langle 4-|6+7| 4-\rangle C_{0}^{(a)}\left(s_{67}, m^{2}\right)\right.
\end{aligned}
$$




$$
\begin{aligned}
&+ {\left[\left(-\frac{1}{2}+\frac{s_{67}}{\langle 4-|6+7| 4-\rangle}\right)\langle 6-|4+7| 2-\rangle\right.} \\
&+\left.\frac{1}{2} \frac{s_{467}}{\langle 4-|6+7| 4-\rangle}\langle 64\rangle[42]\right] B_{0}^{(a)}\left(s_{67}, m^{2}\right) \\
&-\frac{1}{\langle 4-|6+7| 4-\rangle}\left(s_{67}\langle 6-|4+7| 2-\rangle+s_{467}\langle 64\rangle[42]\right) B_{0}^{(b)}\left(m^{2}\right) \\
&\left.+\frac{1}{2} \frac{\langle 64\rangle[42]}{\langle 4-|6+7| 4-\rangle} A_{0}\left(m^{2}\right)-s_{467} \frac{\langle 64\rangle[42]}{\langle 4-|6+7| 4-\rangle} C_{0}^{(-2 \varepsilon)}\left(s_{67}, m^{2}\right)\right\} \\
& B_{W b, \text { loop }}^{[2]}\left(p_{4}^{+}, p_{6}^{-}, p_{7}^{+}, p_{8}^{-}\right)= \\
& \quad-2[47]\langle 6-|4+7| 2-\rangle\left(s_{67} C_{0}^{(b)}\left(s_{67}\right)+\frac{3}{2} B_{0}^{(c)}\left(s_{67}\right)\right) .
\end{aligned}
$$

For the helicity configuration $p_{4}^{+}, p_{6}^{-}, p_{7}^{+}, p_{8}^{+}$we obtain

$$
\begin{aligned}
& B_{W b, \text { loop }}^{[1]}\left(p_{4}^{+}, p_{6}^{-}, p_{7}^{+}, p_{8}^{+}\right)= \\
& \quad-2 m[74]\left[\langle 62\rangle\langle 4-|6+7| 4-\rangle C_{0}^{(a)}\left(s_{67}, m^{2}\right)\right. \\
& \quad+\frac{1}{\langle 4-|6+7| 4-\rangle}\left(s_{67}\langle 62\rangle-\frac{1}{2}\langle 67\rangle[74]\langle 42\rangle\right) B_{0}^{(a)}\left(s_{67}, m^{2}\right) \\
& \quad-\frac{1}{\langle 4-|6+7| 4-\rangle}\left(s_{67}\langle 62\rangle+\langle 64\rangle\langle 4+|6+7| 2+\rangle\right) B_{0}^{(b)}\left(m^{2}\right) \\
& \left.\quad+\frac{1}{2} \frac{\langle 64\rangle\langle 4+|6+7| 2+\rangle}{s_{467}\langle 4-|6+7| 4-\rangle} A_{0}\left(m^{2}\right)+\frac{\langle 2-|6+7| 4-\rangle\langle 46\rangle}{\langle 4-|6+7| 4-\rangle} C_{0}^{(-2 \varepsilon)}\left(s_{67}, m^{2}\right)\right] \\
& B_{W b, \text { loop }}^{[2]}\left(p_{4}^{+}, p_{6}^{-}, p_{7}^{+}, p_{8}^{+}\right)= \\
& \quad-2 m[74]\langle 26\rangle\left(s_{67} C_{0}^{(b)}\left(s_{67}\right)+\frac{3}{2} B_{0}^{(c)}\left(s_{67}\right)\right) .
\end{aligned}
$$

The expressions for the standard scalar integrals are collected in Appendix B. The ultraviolet (UV) renormalization is discussed in the next section.

Finally, we need the real emission amplitudes with one additional gluon. These are listed in Eqs. (4.6-4.9), and correspond to the process

$$
A_{W g}: 0 \rightarrow t\left(p_{8}\right)+\bar{b}\left(p_{4}\right)+g\left(p_{5}\right)+d\left(p_{6}\right)+\bar{u}\left(p_{7}\right) .
$$

The color decomposition reads

$$
A_{W g, \text { real }}=T_{84}^{5} \delta_{67} A_{W g, \text { real }}^{[1]}+\delta_{84} T_{67}^{5} A_{W g, \text { real }}^{[2]} .
$$

These amplitudes have been calculated in Chapter 2. For convenience we repeat them here. We have

$$
A_{W g, \text { real }}^{[1]}=\frac{g e^{2} V_{u d}^{*} V_{t b}}{2 \sin ^{2} \theta_{W}} \cdot \frac{(-i) 2 \sqrt{2}}{s_{67}-m_{W}^{2}} \frac{B_{W g, \text { real }}^{[1]}}{\sqrt{-\langle 2-|4+5+6+7| 2-\rangle}},
$$




$$
A_{W g, \text { real }}^{[2]}=\frac{g e^{2} V_{u d}^{*} V_{t b}}{2 \sin ^{2} \theta_{W}} \cdot \frac{(-i) 2 \sqrt{2}}{s_{567}-m_{W}^{2}} \frac{B_{W g, \text { real }}^{[2]}}{\sqrt{-\langle 2-|4+5+6+7| 2-\rangle}} .
$$

As reference momentum for the massive spinor we have chosen $q=p_{2}$. The nonvanishing amplitudes are

$$
\begin{aligned}
& B_{W g, \text { real }}^{[1]}\left(p_{4}^{+}, p_{5}^{+}, p_{6}^{-}, p_{7}^{+}, p_{8}^{-}\right) \\
& =\frac{\langle 6-|4+5+7| 2-\rangle}{\langle 65\rangle}\left(\frac{\langle 6-|4+5| 7-\rangle}{\langle 45\rangle}+\frac{[74]\langle 6-|4+7| 5-\rangle}{s_{467}-m^{2}}\right), \\
& B_{W g, \text { real }}^{[1]}\left(p_{4}^{+}, p_{5}^{+}, p_{6}^{-}, p_{7}^{+}, p_{8}^{+}\right) \\
& \quad=-\frac{m\langle 26\rangle}{\langle 65\rangle}\left(\frac{\langle 6-|4+5| 7-\rangle}{\langle 45\rangle}+\frac{[74]\langle 6-|4+7| 5-\rangle}{s_{467}-m^{2}}\right) \\
& B_{W g, \text { real }}^{[1]}\left(p_{4}^{+}, p_{5}^{-}, p_{6}^{-}, p_{7}^{+}, p_{8}^{-}\right) \\
& =\frac{[74]}{[54]\left(s_{467}-m^{2}\right)}\left(\langle 5-|4+6+7| 2-\rangle[47]\langle 76\rangle+m^{2}[24]\langle 56\rangle\right), \\
& B_{W g, \text { real }}^{[1]}\left(p_{4}^{+}, p_{5}^{-}, p_{6}^{-}, p_{7}^{+}, p_{8}^{+}\right) \\
& \quad=-\frac{m}{s_{467}-m^{2}} \frac{[47]}{[45]}(\langle 25\rangle\langle 67\rangle[74]+\langle 56\rangle\langle 2-|5+6+7| 4-\rangle), \\
& B_{W g, \text { real }}^{[2]}\left(p_{4}^{+}, p_{5}^{+}, p_{6}^{-}, p_{7}^{+}, p_{8}^{-}\right)=\frac{\langle 6-|4+5+7| 2-\rangle\langle 6-|5+7| 4-\rangle}{\langle 56\rangle\langle 75\rangle}, \\
& B_{W g, \text { real }}^{[2]}\left(p_{4}^{+}, p_{5}^{+}, p_{6}^{-}, p_{7}^{+}, p_{8}^{+}\right)=\frac{m\langle 62\rangle\langle 6-|5+7| 4-\rangle}{\langle 56\rangle\langle 75\rangle}, \\
& B_{W g, \text { real }}^{[2]}\left(p_{4}^{+}, p_{5}^{-}, p_{6}^{-}, p_{7}^{+}, p_{8}^{-}\right)=\frac{[74]\langle 2+|(4+5+6+7)(5+6)| 7-\rangle}{[57][56]} \\
& B_{W g, \text { real }}^{[2]}\left(p_{4}^{+}, p_{5}^{-}, p_{6}^{-}, p_{7}^{+}, p_{8}^{+}\right)=\frac{m[47]\langle 2-|5+6| 7-\rangle}{[57][56]} .
\end{aligned}
$$

The matrix element squared is given by

$$
\left|A_{W g, \text { Real }}\right|^{2}=\frac{1}{2} N_{C}\left(N_{C}^{2}-1\right)\left(\left|A_{W g, \text { Real }}^{[1]}\right|^{2}+\left|A_{W g, \text { Real }}^{[2]}\right|^{2}\right) .
$$

There are no interference terms between $A_{W g, \text { Real }}^{[1]}$ and $A_{W g, \text { Real }}^{[2]}$.

\subsubsection{Conversion between schemes and scheme independence}

The one-loop amplitudes presented in the previous section have been calculated in a four-dimensional scheme. They differ from the corresponding amplitudes in the 
't Hooft-Veltman scheme by finite terms. These finite terms are either of ultraviolet (UV) or infrared (IR) origin and result from expressions of the form $\varepsilon / \varepsilon$. To obtain the unique and correct result, one proceeds through the following steps:

- The bare one-loop amplitudes may contain UV-divergences. With the help of a specific regularization scheme these divergences are isolated and removed by renormalization. The explicit form of the renormalization depends on the chosen renormalization scheme.

- The specific combination of regularization and renormalization scheme may break certain Ward identities. These Ward identities have to be restored through finite renormalizations. The required finite renormalizations are universal, i.e. they do not depend on the process under consideration. After this step all finite parts of UV-origin are uniquely fixed.

- In addition, QCD amplitudes may contain IR-divergences. Unitarity requires that we employ the same regularization scheme in the phase space integral over the unresolved real emission part as in the one-loop integral.

Alternatively, since the structure of the IR-divergences is universal, we may derive simple formulas, which relate the finite parts specific to a certain regularization scheme to the ones of another scheme. We can therefore convert a one-loop amplitude calculated in one scheme to the corresponding amplitude calculated in another scheme.

We will discuss the three steps in detail for the case at hand. To start, let us briefly summarize the properties of the 't Hooft-Veltman scheme and the four-dimensional scheme. The 't Hooft-Veltman scheme treats unobserved particles (particles in loops and unresolved partons in the real emission part) in $D=4-2 \varepsilon$ dimensions. Observed particles are taken in four dimensions. $\gamma_{5}$ is a four-dimensional object in the 't HooftVeltman scheme, anti-commuting with the first four Dirac matrices and commuting with the remaining ones.

The four-dimensional scheme is specified in simple terms by the fermion propagators

$$
i \frac{p_{(4)}+m 1_{(4)}}{p_{(D)}^{2}-m^{2}} .
$$

Four-dimensional Dirac-matrices occur in the numerator, whereas $D$-dimensional quantities occur in the denominator. Two adjacent Dirac-matrices in the numerator are contracted as

$$
\not p_{(4)} p_{(4)}=\left(p_{(D)}^{2}-p_{(-2 \varepsilon)}^{2}\right) \cdot 1_{(4)},
$$

which can be interpreted as the statement " $D$ is effectively larger then 4 ". $p_{(D)}^{2}$ can cancel a propagator, whereas $p_{(-2 \varepsilon)}^{2}$ will give rise to an integral in $6-2 \varepsilon$ dimensions. 
It should be kept in mind that the specification given here is just a simple prescription relevant to practical calculations. The scheme is rigorously defined in Ref. [86].

$A_{W b \text {, loop }}$ contains ultraviolet and infrared divergences. Ultraviolet divergences are removed after renormalization of the quark fields

$$
\psi_{\text {bare }}=Z_{\psi}^{1 / 2} \psi_{\text {ren }}
$$

The renormalized amplitude is obtained as

$$
A_{W b, \text { loop }, \text { ren }}=\left(Z_{\psi}^{1 / 2}\right)^{4} A_{W b, \text { loop }, \text { bare }} .
$$

We have to renormalize the fields such that the residuum of the propagators is 1 . For light quarks the appropriate renormalization constant is 1 , due to a cancellation of UV- and IR- divergent parts. In more detail we have

$$
\begin{aligned}
& Z_{\psi, \text { onshell }, F D}^{1 / 2}=1+\frac{1}{2} \frac{g^{2}}{(4 \pi)^{2}} C_{F}\left(-\Delta_{U V}+\Delta_{I R}\right) \\
& Z_{\psi, \text { onshell }, H V}^{1 / 2}=1+\frac{1}{2} \frac{g^{2}}{(4 \pi)^{2}} C_{F}\left(-\Delta_{U V}+1_{U V}+\Delta_{I R}-1_{I R}\right)
\end{aligned}
$$

where $\Delta=1 / \varepsilon-\gamma+\ln 4 \pi$. Here we have indicated with a subscript UV or IR the origins of the divergent parts as well as the origin of additional finite terms which arise from a cancellation of $1 / \varepsilon$-terms with terms of order $\varepsilon$. For massive quarks we have

$$
\begin{aligned}
& Z_{\psi, \text { onshell }, F D}^{1 / 2}=1+\frac{1}{2} \frac{g^{2}}{(4 \pi)^{2}} C_{F}\left(-3 \Delta_{U V}-5+3 \ln \frac{m^{2}}{\mu^{2}}\right) \\
& Z_{\psi, \text { onshell }, H V}^{1 / 2}=1+\frac{1}{2} \frac{g^{2}}{(4 \pi)^{2}} C_{F}\left(-3 \Delta_{U V}+1_{U V}-5+3 \ln \frac{m^{2}}{\mu^{2}}\right) .
\end{aligned}
$$

After renormalization of the quark field we have

$$
\begin{aligned}
& B_{W b, \text { loop,ren }, F D}^{[1]}=B_{W b, \text { loop, bare }, F D}^{[1]}+\frac{1}{2}\left(-3 \Delta-5+3 \ln \frac{m^{2}}{\mu^{2}}\right) B_{W b, \text { born }}^{[1]} \\
& B_{W b, \text { loop,ren }, F D}^{[2]}=B_{W b, \text { loop }, \text { bare }, F D}^{[2]} .
\end{aligned}
$$

The only divergences left in the renormalized amplitudes are of infrared origin. The infrared divergent parts are given by

$$
\begin{aligned}
& \left.B_{W b, \text { loop }}^{[1]}\right|_{I R}=-2 \Delta\left(\frac{1}{2} \Delta+\frac{1}{2} \gamma-\frac{1}{2} \ln 4 \pi+\frac{5}{4}-\ln \frac{m^{2}-s_{67}}{\mu^{2}}+\frac{1}{2} \ln \frac{m^{2}}{\mu^{2}}\right) B_{W b, \text { born }} \\
& \left.B_{W b, \text { loop }}^{[2]}\right|_{I R}=-2 \Delta\left(\Delta+\gamma-\ln 4 \pi+\frac{3}{2}-\ln \frac{-s_{67}}{\mu^{2}}\right) B_{W b, b o r n} .
\end{aligned}
$$


We now turn our attention to Ward identities and finite renormalizations. In the 't Hooft-Veltman scheme $\gamma_{5}$ anti-commutes with the first four Dirac matrices and commutes with the remaining ones. This treatment violates a Ward identity, which has to be restored by a finite renormalization. Explicitly, one splits the left-handed interaction into a vector $\left(\gamma_{\mu}\right)$ and axial-vector $\left[\Gamma_{\mu 5}=1 / 2\left(\gamma_{\mu} \gamma_{5}-\gamma_{5} \gamma_{\mu}\right)\right]$ part.

$$
\gamma_{\mu} \frac{1}{2}\left(1-\gamma_{5}\right)=\frac{1}{2} \gamma_{\mu}-\frac{1}{4}\left(\gamma_{\mu} \gamma_{5}-\gamma_{5} \gamma_{\mu}\right)
$$

The Ward identity is restored by a finite renormalization of the axial-vector coupling:

$$
\begin{aligned}
\Gamma_{\mu 5}^{\text {bare }} & =Z_{\text {axial }, H V, \text { fin }} \Gamma_{\mu 5}^{\text {renorm }} \\
Z_{\text {axial }, H V, f i n} & =1+4 \frac{g^{2}}{(4 \pi)^{2}} C_{F} .
\end{aligned}
$$

There is some freedom in how we continue the left-handed coupling in the 't HooftVeltman scheme to $D$ dimensions. For example, the expressions

$$
\gamma_{\mu} \frac{1}{2}\left(1-\gamma_{5}\right), \frac{1}{2}\left(1+\gamma_{5}\right) \gamma_{\mu}, \frac{1}{2}\left(1+\gamma_{5}\right) \gamma_{\mu} \frac{1}{2}\left(1-\gamma_{5}\right), \frac{1}{2} \gamma_{\mu}-\frac{1}{4}\left(\gamma_{\mu} \gamma_{5}-\gamma_{5} \gamma_{\mu}\right)
$$

all agree in four dimensions, but differ in $D$ dimensions. Of course this difference is compensated by the appropriate finite renormalization. $Z_{\text {axial }, H V, f i n}$ corresponds to the choice $-1 / 4\left(\gamma_{\mu} \gamma_{5}-\gamma_{5} \gamma_{\mu}\right)$.

The four-dimensional scheme violates a Ward identity as well, which is restored by a finite renormalization of the left-handed coupling $\Gamma_{\text {left }}=1 / 2 \gamma_{\mu}\left(1-\gamma_{5}\right)$,

$$
\begin{aligned}
\Gamma_{\text {left }}^{\text {bare }} & =Z_{E W, F D \text { fin }} \Gamma_{\text {left }}^{\text {renorm }} \\
Z_{E W, F D, f i n} & =1+\frac{g^{2}}{(4 \pi)^{2}} C_{F} .
\end{aligned}
$$

After the finite renormalization the renormalized amplitudes

$$
\begin{aligned}
& A_{W b, \text { loop }, \text { ren }, H V}=\left(Z_{\psi, H V}^{1 / 2}\right)^{4} A_{W b, l o o p, H V} \\
& A_{W b, \text { loop }, \text { ren }, F D}=\left(Z_{\psi, F D}^{1 / 2}\right)^{4}\left(Z_{E W, F D, f i n}\right)^{-2} A_{W b, l o o p, F D}
\end{aligned}
$$

agree up to terms resulting from a different treatment of the infrared divergences. The axial-vector coupling in the 't Hooft-Veltman scheme has been renormalized according to

$$
\Gamma_{\mu 5}^{\text {bare }}=Z_{\text {axial }, H V, f i n} \Gamma_{\mu 5}^{r e n o r m}
$$


In detail, we have for the finite parts of UV-origin in terms of $g^{2} /(4 \pi)^{2} C_{F} B^{\text {Born }}$

\begin{tabular}{c|c|c|c}
\hline \hline & $F D$ & $\mathrm{HV}_{\text {vector }}$ & $\mathrm{HV}_{\text {axial }}$ \\
\hline \hline$B_{\text {Wb,loop,bare }}$ & 0 & -2 & +2 \\
\hline$Z_{\text {coupling }}^{-1}$ & -1 & 0 & -4 \\
\hline$Z_{\psi}$ & 0 & +1 & +1 \\
\hline \hline Sum & -1 & -1 & -1 \\
\hline \hline
\end{tabular}

The remaining differences are due to finite terms of infrared origin. Due to the universal structure of the infrared divergences we may relate the amplitudes calculated in the four-dimensional scheme to the ones in the 't Hooft-Veltman scheme [87, 65, 5]. The relations are

$$
\begin{aligned}
& B_{W b, \text { loop }, H V}^{[1]}=B_{W b, \text { loop }, F D}^{[1]}-\frac{1}{2} B_{W b, \text { Born }}^{[1]} \\
& B_{W b, \text { loop }, H V}^{[2]}=B_{W b, \text { loop }, F D}^{[2]}-B_{W b, \text { Born }}^{[1]}
\end{aligned}
$$

This completes our discussion on scheme-independence. In summary, we are able to perform the calculation in a four-dimensional scheme and to obtain from this result the amplitudes in the 't Hooft-Veltman scheme through simple and universal relations.

We would like to comment on the original formulation of dimensional reduction. Dimensional reduction $[88,89]$ differs from the four-dimensional scheme by use of the relation

$$
\not p_{(4)} p_{(4)}=p_{(D)}^{2} \cdot 1_{(4)}
$$

which can be interpreted as " $\mathrm{D}$ is smaller than 4". The results in dimensional reduction can be easily obtained from ours by dropping the terms $C_{0}^{(-2 \varepsilon)}$. The $C_{0}^{(-2 \varepsilon)}$-terms yield finite terms related to UV-divergences. We note that the results in dimensional reduction cannot be related to ours, nor to the 't Hooft-Veltman scheme by a finite renormalization, since the term with $C_{0}^{(-2 \varepsilon)}$ in $B_{W b, l o o p}^{[1]}$ is not proportional to $B_{W b, b o r n}$. Therefore the naive approach of dimensional reduction is not consistent. The situation may be cured at the expense of introducing additional scalar ghost particles [90], however this spoils the calculational simplicity of the scheme. From a calculational point of view we prefer the scheme defined in Ref. [86], since $6-2 \varepsilon$-dimensional integrals are rather "inexpensive" to evaluate.

\subsubsection{Spin observables}

The helicity amplitudes contain the complete spin information for the single-top-quark processes. In the old-fashioned method, spin observables are calculated by inserting 
the spin projection operator

$$
u(p, s) \bar{u}(p, s)=(\not p+m) \frac{1}{2}\left(1+\gamma_{5} \not\right),
$$

into the matrix element squared. In Eq. (4.74) $s$ denotes a spin four-vector with $s^{2}=-1$, and $p \cdot s=0$. In the rest frame of the particle the spatial components of $s$ point in the same direction as the spin of the particle [42]. To make contact with this formulation, we first introduce the spin density matrix [91] in the basis of Eq. (2.12):

$$
\rho=\left(\begin{array}{cc}
A\left(\ldots, p^{+}, \ldots\right) A^{*}\left(\ldots, p^{+}, \ldots\right) & A\left(\ldots, p^{+}, \ldots\right) A^{*}\left(\ldots, p^{-}, \ldots\right) \\
A\left(\ldots, p^{-}, \ldots\right) A^{*}\left(\ldots, p^{+}, \ldots\right) & A\left(\ldots, p^{-}, \ldots\right) A^{*}\left(\ldots, p^{-}, \ldots\right)
\end{array}\right) .
$$

In addition we need the projection operator Eq. (4.74) in the basis of Eq. (2.12). This one is obtained as

$$
P=\frac{1}{2} \frac{1}{2 p q}\left(\begin{array}{ll}
-\langle q-|(\not p+m)(1-\not)| q-\rangle & \langle q-|(\not p+m)(1-\not)| q+\rangle \\
-\langle q+|(\not p+m)(1-\not)| q-\rangle & \langle q+|(\not p+m)(1-\not)| q+\rangle
\end{array}\right) .
$$

Spin observables are then calculated as

$$
\operatorname{Tr}(P \cdot \rho)
$$

Note that the entries of the matrices are complex numbers, and that the spin vector $s$ enters only through the matrix $P$. It is easily verified that this expression agrees with the one obtained from Eq. (4.74). The spin summed result is recovered by replacing $P$ in Eq. (4.77) with the unit matrix.

\subsection{Numerical results}

The inclusive NLO cross sections for $s$ - and $t$-channel production of single-top-quarks were published in Refs. [75] and [77], respectively. After we choose our numerical inputs, we compare to these older calculations, and update the inclusive cross sections using newer parton distribution functions (PDFs).

In order to make a definite comparison to the older calculations, we reevaluate all results with the following parameters: For the mass of the $W$ boson we use $m_{W}=80.4$ $\mathrm{GeV}$. For the top-quark mass we take $m_{t}=175 \mathrm{GeV}$. In LO calculations we use CTEQ5L PDFs [92]. In NLO calculations we use CTEQ5M1 PDFs with 2-loop running of $\alpha_{s}$. We define the electroweak coupling by $g^{2}=8 G_{F} M_{W}^{2} / \sqrt{2}$, with Fermi coupling constant of $G_{F}=1.16639 \times 10^{-5} \mathrm{GeV}^{-2}$. We consider $p \bar{p}$ collisions with center-ofmomentum energy $\sqrt{S}=1.8,1.96$, or $2.0 \mathrm{TeV}$ (Tevatron), and $p p$ collisions with a center-of-momentum energy $\sqrt{S}=14 \mathrm{TeV}$ (LHC). 
Unlike the massive dipole formalism, the phase space slicing method of two cutoffs depends on explicit parameters $\delta_{s}$ and $\delta_{c}$. In Figures 4.3 and 4.4 we show the $s$ - and $t$-channel inclusive cross sections as a function of $\delta_{s}=300 \times \delta_{c}$. The logarithmic dependence cancels in the sum of the two- and three-body contributions and leaves terms proportional to $\delta_{s}$ and $\delta_{c}$. By taking $\delta_{s}<$ a few $\times 10^{-3}$, the cross sections converge to the updated analytic results.

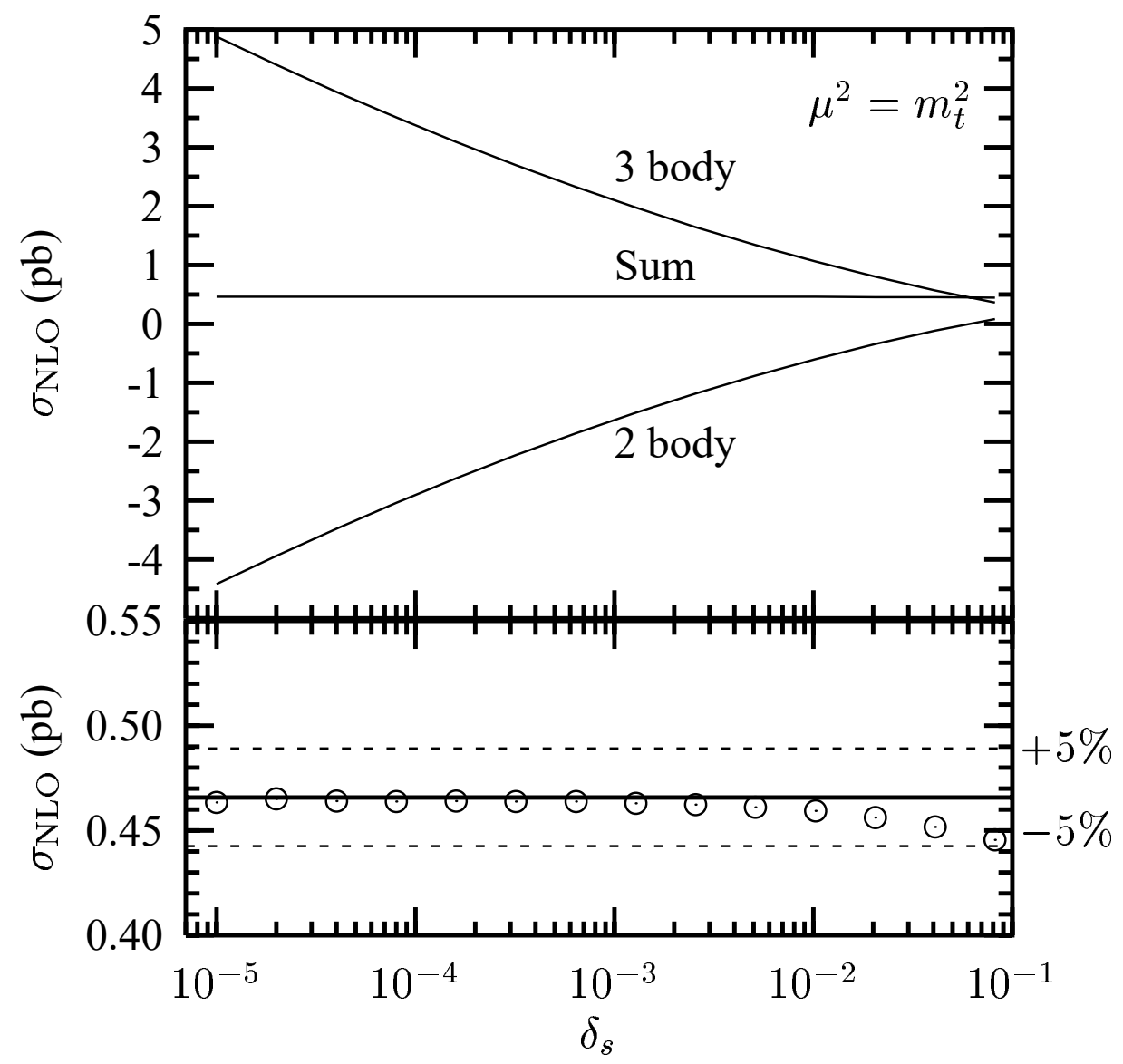

Figure 4.3: The next to leading order single-top-quark total cross section for the $s$ channel at a $\sqrt{S}=2 \mathrm{TeV}$ proton-antiproton machine. The two- and three- body contributions, together with their sum, are shown as a function of the soft cutoff $\delta_{s}$. The bottom enlargement shows the sum (open circles) relative to $\pm 5 \%$ (dotted lines) of the analytic result (solid line).

The LO and NLO cross sections for $s$-channel and $t$-channel single-top-quark production are listed in picobarns in Tables 4.1 and 4.2. Since the Tevatron is a $p \bar{p}$ collider, the cross sections for top-quark $(t)$ and antitop-quark $(\bar{t})$ production are the same. The LHC is a $p p$ collider, and hence the $t$ and $\bar{t}$ cross sections are listed sepa- 


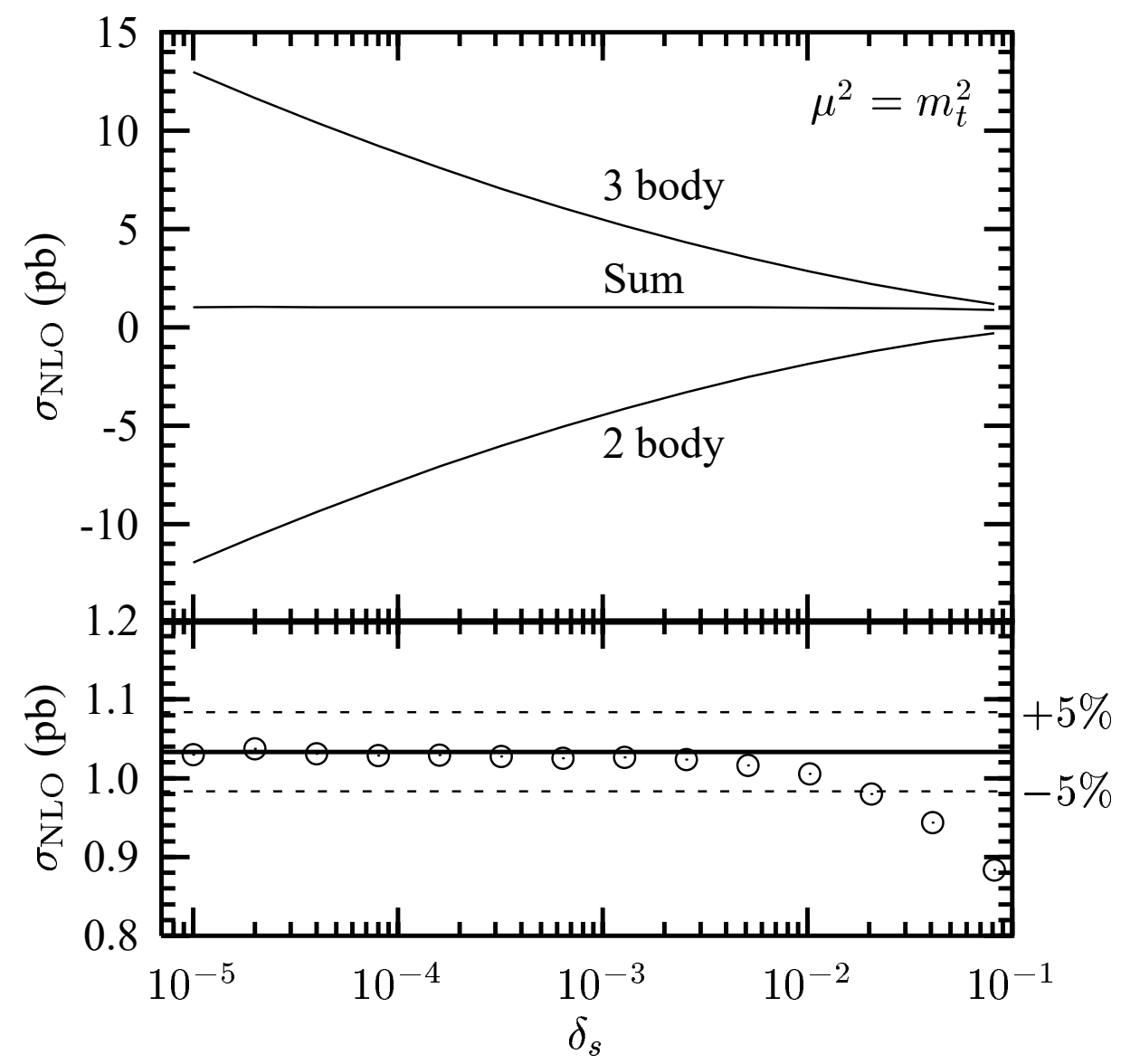

Figure 4.4: The next to leading order single-top-quark total cross section for the $t$ channel at a $\sqrt{S}=2 \mathrm{TeV}$ proton-antiproton machine. The two- and three- body contributions, together with their sum, are shown as a function of the soft cutoff $\delta_{s}$. The bottom enlargement shows the sum (open circles) relative to $\pm 5 \%$ (dotted lines) of the analytic result (solid line).

rately. Factorization $\left(\mu_{F}\right)$ and renormalization $\left(\mu_{R}\right)$ scales are set equal. In Table 4.1 all scales are set to the top mass $m_{t}$. In Table 4.2 the $s$-channel cross sections are calculated using the invariant mass of the top-quark/antibottom-jet pair for the scale, $\mu^{2}=M_{t \bar{b}}^{2}=\left(P_{t}+P_{\bar{b}}\right)^{2}$, where $P_{t}$ and $P_{\bar{b}}$ are the four-momenta of the top quark and antibottom jet, respectively. ${ }^{2}$ The $t$-channel cross section uses the double deepinelastic-scattering (DDIS) scales, $\mu_{l}^{2}=Q^{2}=-\left(p_{b}-p_{t}\right)^{2}$ for the light-quark line and $\mu_{h}^{2}=Q^{2}+m_{t}^{2}=-\left(p_{u}-p_{d}\right)^{2}+m_{t}^{2}$ for the heavy-quark line, where $Q^{2}$ is the virtuality

\footnotetext{
${ }^{2}$ The choice of jet definition induces a cone-size dependence in the scale that always enters the cross section at one higher order in QCD. We have confirmed that this effect is numerically less than the overall scale uncertainty for any infrared-safe jet definition.
} 


\begin{tabular}{c|r|c|c}
\hline \hline Process & $\sqrt{S}$ & $\sigma_{L O}(\mathrm{pb})$ & $\sigma_{N L O}(\mathrm{pb})$ \\
\hline \hline$s$-channel & $1.8 \mathrm{TeV} p \bar{p}(t)$ & 0.259 & $0.380 \pm 0.002$ \\
\hline & $1.96 \mathrm{TeV} p \bar{p}(t)$ & 0.304 & $0.447 \pm 0.002$ \\
\hline & $2 \mathrm{TeV} p \bar{p}(t)$ & 0.315 & $0.463 \pm 0.002$ \\
\hline & $14 \mathrm{TeV} p p(t)$ & 4.53 & $6.55 \pm 0.03$ \\
\hline \hline & $14 \mathrm{TeV} p p(\bar{t})$ & 2.74 & $4.07 \pm 0.02$ \\
\hline & $1.8 \mathrm{TeV} p \bar{p}(t)$ & 0.648 & $0.702 \pm 0.003$ \\
\hline & $1.96 \mathrm{TeV} p \bar{p}(t)$ & 0.883 & $0.959 \pm 0.002$ \\
\hline & $2 \mathrm{TeV} p \bar{p}(t)$ & 0.948 & $1.029 \pm 0.004$ \\
\hline & $14 \mathrm{TeV} p p(t)$ & 144.8 & $152.6 \pm 0.6$ \\
\hline \hline & $14 \mathrm{TeV} p p(\bar{t})$ & 83.4 & $90.0 \pm 0.5$ \\
\hline
\end{tabular}

Table 4.1: LO and NLO cross sections for single-top-quark production at the Tevatron and LHC for $m_{t}=175 \mathrm{GeV}$. Cross sections are evaluated with CTEQ5L and CTEQ5M1 PDFs, and all scales set to $m_{t}$. Errors include only Monte Carlo statistics.

of the exchanged $W$ boson (valid through NLO), and $p_{i}$ are the four-momenta of the partons in Eq. (4.1). All LO cross sections are identical to those in Refs. [75] and [77] once updated to the CTEQ5 PDFs.

We compare the NLO $s$-channel cross sections to a recoding of Refs. [93] and [75]. The results agree to within $1 \%$ for all scale choices. Of note is our use of $M_{t \bar{b}}$ for the scale in Table 4.2 rather than $Q^{2}$, the virtuality of the $W$, which was used in Ref. [75]. These two scales are identical at LO and in initial-state corrections, but differ by the emitted gluon in final state corrections. Since we are interested in making cuts based on observables, such as $M_{t \bar{b}}$, we choose this as the scale. While the central value of any of the scale choices is very similar, the uncertainty is slightly larger at NLO using $m_{t}$ or $M_{t \bar{b}}$. In particular, if we vary the scales between $M_{t \bar{b}} / 2$ and $2 M_{t \bar{b}}$ (or $m_{t} / 2$ and $\left.2 m_{t}\right)$, we find the $s$-channel cross section varies by $+7.8-6.9 \%$ at LO, and $+5.7-5.0 \%$ $(+5.5-4.6 \%)$ at NLO. In contrast, using a scale of $Q^{2}$, as in Ref. [75], would predict a NLO scale uncertainty of $\pm 4 \%$. Given that we can probe a more restricted phase space with cuts, we take the conservative view that $\pm 5.7 \%$ is an appropriate estimate of the NLO scale uncertainty at the Tevatron when looking at exclusive final states. At the LHC the scale uncertainty is less than $\pm 2 \%$.

At LO and NLO, but not NNLO, color conservation forbids the exchange of a gluon between the light- and heavy-quark lines. Hence, the $t$-channel process may be factorized at NLO into two independent corrections that each resemble deep-inelasticscattering. The NLO t-channel cross sections in Ref. [77] were calculated using the 


\begin{tabular}{c|r|c|c}
\hline \hline Process & $\sqrt{S}$ & $\sigma_{L O}(\mathrm{pb})$ & $\sigma_{N L O}(\mathrm{pb})$ \\
\hline \hline$s$-channel & $1.8 \mathrm{TeV} p \bar{p}(t)$ & 0.244 & $0.377 \pm 0.002$ \\
\hline & $1.96 \mathrm{TeV} p \bar{p}(t)$ & 0.287 & $0.442 \pm 0.002$ \\
\hline & $2 \mathrm{TeV} p \bar{p}(t)$ & 0.297 & $0.459 \pm 0.002$ \\
\hline & $14 \mathrm{TeV} p p(t)$ & 4.612 & $6.56 \pm 0.03$ \\
\hline & $14 \mathrm{TeV} p p(\bar{t})$ & 2.788 & $4.09 \pm 0.02$ \\
\hline \hline -channel & $1.8 \mathrm{TeV} p \bar{p}(t)$ & 0.735 & $0.725 \pm 0.003$ \\
\hline & $1.96 \mathrm{TeV} p \bar{p}(t)$ & 0.996 & $0.990 \pm 0.002$ \\
\hline & $2 \mathrm{TeV} p \bar{p}(t)$ & 1.068 & $1.062 \pm 0.004$ \\
\hline & $14 \mathrm{TeV} p p(t)$ & 152.7 & $155.9 \pm 0.6$ \\
\hline \hline & $14 \mathrm{TeV} p p(\bar{t})$ & 86.1 & $90.7 \pm 0.5$ \\
\hline
\end{tabular}

Table 4.2: $L O$ and NLO cross sections for single-top-quark production at the Tevatron and LHC for $m_{t}=175 \mathrm{GeV}$. Cross sections are evaluated with CTEQ5L and CTEQ5M1 PDFs, and $M_{t \bar{b}}$ or the DDIS scales $\left(\mu_{l}=Q^{2}, \mu_{h}=Q^{2}+m_{t}^{2}\right)$, for $s$-channel or t-channel, respectively. Errors include only Monte Carlo statistics.

scales suggested by this relation to double deep-inelastic-scattering. When using the DDIS scales, our results in Table 4.2 match the updated Ref. [77] to better than $0.3 \%$ at the Tevatron, but are larger by $2.9 \%$ at the LHC. The cross sections in Table 4.1 agree with the updated results of Ref. [77] to within $1 \%$ when evaluated at $\mu=m_{t}$. In all cases, the dipole subtraction calculations and phase space slicing calculations agree within the statistical errors.

A subtle issue arises in attempting to ascertain the effect of higher orders by varying the scale. In Ref. [77] only the scales in the vertex and PDFs of the heavy-quark line $(b \rightarrow t)$ were varied because the corrections to the light-quark structure function are small. ${ }^{3}$ However, to the extent this process looks like double deep-inelastic-scattering, we expect a similarly small effect for the heavy-quark corrections as well. Indeed, the results for LO and NLO are nearly identical when using the DDIS scales, but differ significantly when using a fixed scale such as $m_{t}$. In Table 4.3 we show the effects of varying the scales at the Tevatron $(\sqrt{S}=2 \mathrm{TeV})$ together, and separately in the light- and heavy-quark lines. What we see is that varying the scales together at LO, particularly when using $m_{t}$, severely underestimates the NLO correction.

The reason for the underestimate in varying the scales together is a series of accidental cancellations that are driven by the range of proton momentum fraction probed

\footnotetext{
${ }^{3}$ The NLO correction to the light-quark line effectively undoes the extraction of the NLO PDFs from the DIS data.
} 


\begin{tabular}{|c|c|c|c|c|}
\hline & $\sigma_{t}$ & $\mu_{h} \& \mu_{l}$ & $\mu_{h}$ & $\mu_{l}$ \\
\hline $\mathrm{LO}_{t}\left(m_{t}\right)$ & $0.95 \mathrm{pb}$ & $\pm 1 \%$ & $\begin{array}{l}-7.5 \% \\
+5.5 \%\end{array}$ & $\begin{array}{l}+6.7 \% \\
{ }_{-5.8}\end{array}$ \\
\hline $\mathrm{NLO}_{t}\left(m_{t}\right)$ & $1.03 \mathrm{pb}$ & $\pm 2.5 \%$ & $\begin{array}{l}-3.5 \% \\
+4.0 \\
\end{array}$ & $\pm 1 \%$ \\
\hline $\mathrm{LO}_{t}(\mathrm{DDIS})$ & $1.07 \mathrm{pb}$ & ${ }_{-2}^{+0.1 \%}$ & $\begin{array}{l}-7.2 \% \\
+5.2 \% \\
\end{array}$ & $\begin{array}{l}+8.8 \% \\
\end{array}$ \\
\hline $\mathrm{NLO}_{t}(\mathrm{DDIS})$ & $1.06 \mathrm{pb}$ & $\pm 3.5 \%$ & $\begin{array}{l}-3 \% \\
+4 \%\end{array}$ & $\pm 0.6 \%$ \\
\hline
\end{tabular}

Table 4.3: Scale variation of the $L O$ and NLO cross sections for t-channel single-topquark production at the Tevatron $(\sqrt{S}=2 \mathrm{TeV})$. Variation in the light-quark and heavy-quark lines are listed as $\mu_{l}$ and $\mu_{h}$, respectively. Fixed $\left(\mu=m_{t}\right)$, and double deep-inelastic-scattering scales are shown separately.

at the Tevatron. For a top mass of $175 \mathrm{GeV}$ and a machine of around $2 \mathrm{TeV}$, the typical $x \sim 0.1$. For scales also around $100-200 \mathrm{GeV}$, the $b$ PDF happens to increase with increasing scale, whereas the valence quarks decrease. This may be seen in the opposite signs in the last two columns of Table 4.3. The net effect is that to estimate the uncertainty, we must vary the scales independently, and then add them in quadrature. ${ }^{4}$ This leads to a LO uncertainty of $\sim \pm 10 \%$, and a NLO uncertainty for the Tevatron of $\pm 4 \%$. At the LHC, the NLO uncertainty is $\pm 3 \%$. These uncertainties are consistent with expected higher-order corrections, and with both fixed and DDIS scale choices.

While the $s$-channel cross section has only changed by a couple of percent from [75], the $t$-channel cross section is $13 \%$ smaller at the Tevatron than appears in [77]. The shift in the NLO $t$-channel cross section is due to the correction of bugs that appeared in the evolution of the gluon PDF in all of the older MRS and CTEQ NLO PDFs [94]. Because the $b$ PDF is constructed almost entirely out of gluon evolution [77, 95], the effect of the bug is greatly enhanced in all processes where there is a $c$ or $b$ quark in the initial state. The programming bug is corrected in MRS99 (updated) [96] and CTEQ5M1 [92].

The central goal of our calculations is not to recalculate inclusive cross sections, but to provide full momentum and spin dependent distributions with the option for arbitrary cuts. Detailed analyses of the phenomenological issues concerning these distributions will appear in the future. Here we restrict ourselves to a comparison of the results using the phase space slicing method and massive dipole formalism.

For our comparisons we reconstruct jets using a $k_{T}$ cluster algorithm [97] with $\Delta R=1$. We define a simple detector acceptance by assuming that only jets with $p_{T}>20 \mathrm{GeV}$ and $|\eta|<2$ are observed. We calculate all cross sections at the scale

\footnotetext{
${ }^{4}$ The light- and heavy-quark corrections are factorizable through NLO, and hence are only weakly correlated through the evolution of the PDFs.
} 
$\mu=m_{t}=175 \mathrm{GeV}$, and at a $2 \mathrm{TeV} p \bar{p}$ collider.

In Figures 4.5 and 4.6 we present the NLO transverse momentum $p_{T b}$ and pseudorapidity $\eta_{b}$ distributions of the $b$-jet in $s$-channel production of a top quark, with cuts based on the "jet veto" search strategy in Ref. [16]. We accept events where only the top-quark and $b$-jet pass the cuts above, and any additional jets are either soft $\left(p_{T j}<20 \mathrm{GeV}\right)$ or are outside the simple detector $\left(\left|\eta_{j}\right|>2\right)$. The phase space slicing method and massive dipole formalism give identical distributions, even in this region of restricted phase space.

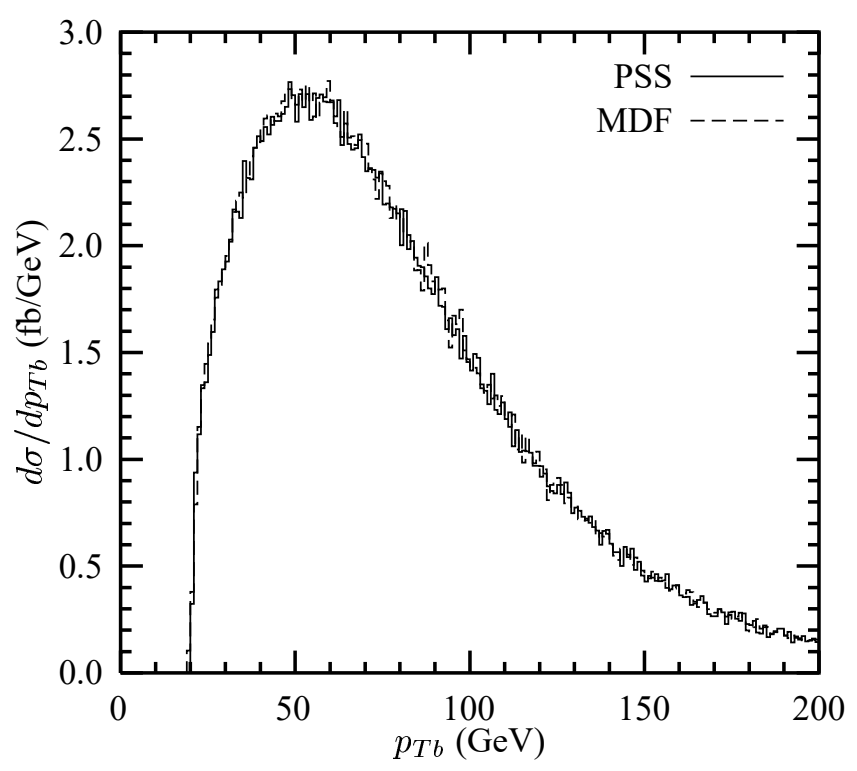

Figure 4.5: Transverse momentum distribution of the b-jet at NLO in s-channel production of a top quark at the Tevatron $(\sqrt{S}=2 \mathrm{TeV})$ after cuts. Phase space slicing (PSS) results (solid) and massive dipole formalism (MDF) results (dashed) are both shown.

In Figures 4.7 and 4.8 we present the NLO transverse momentum $p_{T j_{1}}$ and pseudorapidity $\eta_{j_{1}}$ distributions of the highest- $p_{T}$ jet in $t$-channel production of a top quark, using the same "jet veto" search strategy as above. We accept events where only the top-quark and highest- $p_{T}$ jet pass the cuts above, and any additional jets are either soft $\left(p_{T j_{2}}<20 \mathrm{GeV}\right)$ or are outside the simple detector $\left(\left|\eta_{j_{2}}\right|>2\right)$. The phase space slicing method and massive dipole formalism predict identical distributions at NLO.

\subsection{Conclusions}

We presented three independent calculations of the fully differential production of a single top quark plus one jet at next to leading order in hadronic collisions. At this 


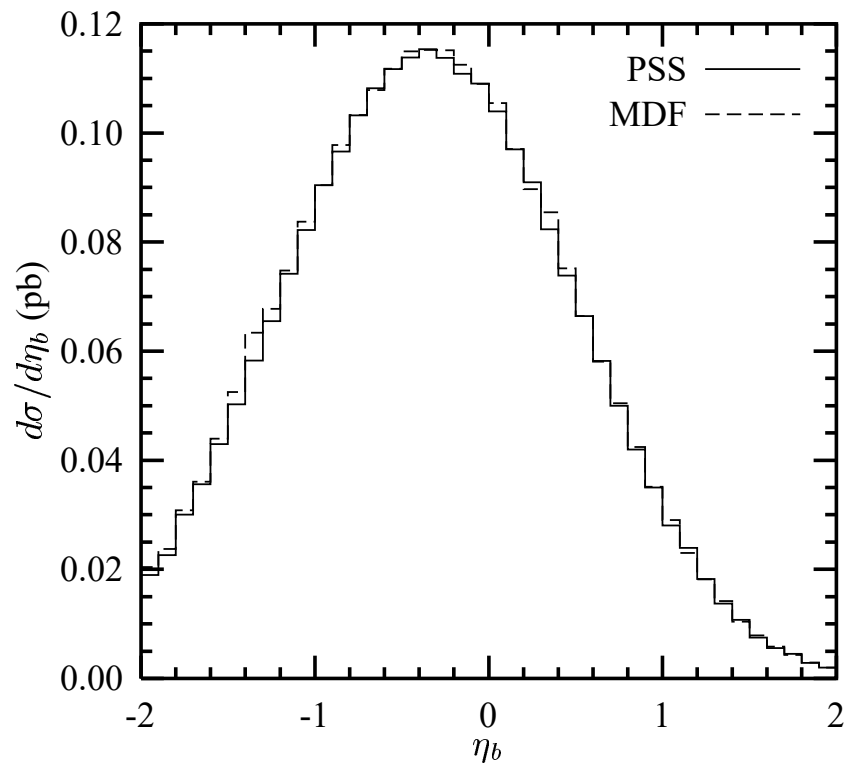

Figure 4.6: Pseudorapidity distribution of the b-jet at NLO in s-channel production of a top quark at the Tevatron $(\sqrt{S}=2 \mathrm{TeV})$ after cuts. Phase space slicing (PSS) results (solid) and massive dipole formalism (MDF) results (dashed) are both shown.

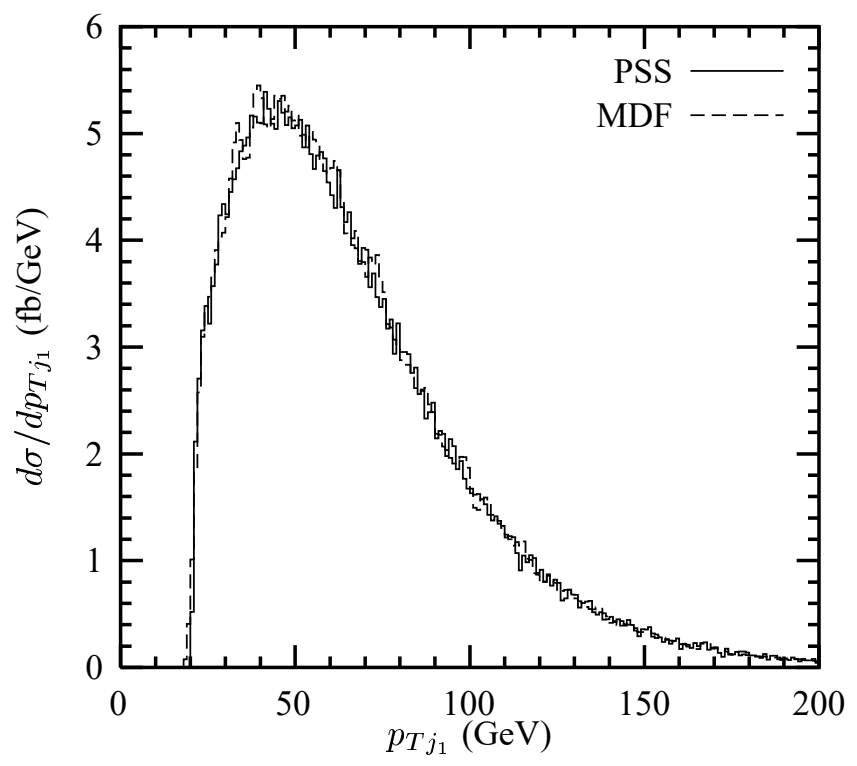

Figure 4.7: Transverse momentum distribution of the highest- $p_{T}$ jet $\left(j_{1}\right)$ at NLO in t-channel production of a top quark at the Tevatron $(\sqrt{S}=2 \mathrm{TeV})$ after cuts. Phase space slicing (PSS) results (solid) and massive dipole formalism (MDF) results (dashed) are both shown. 


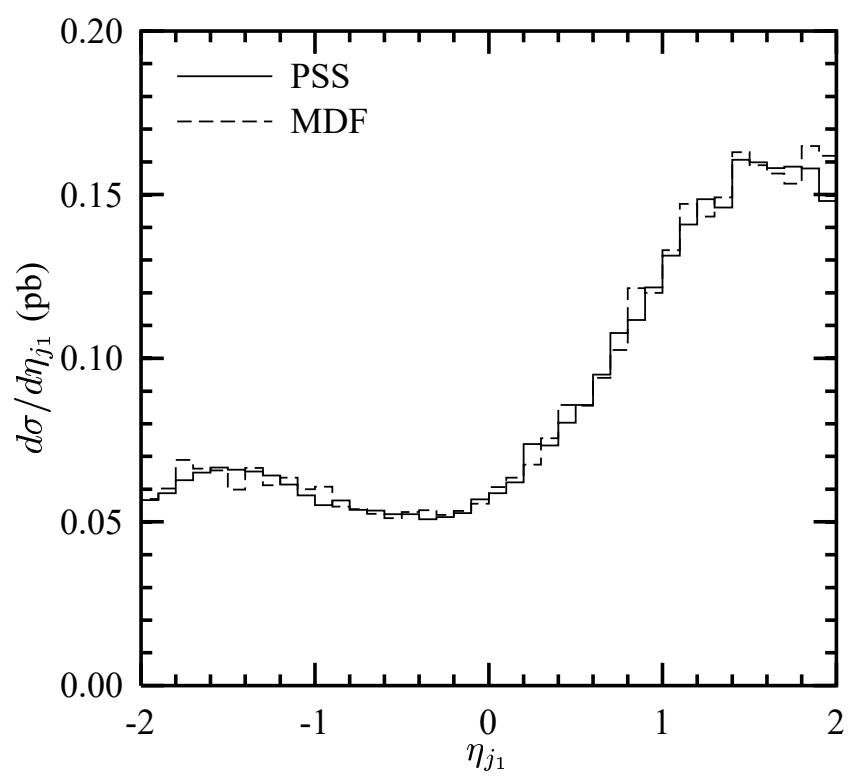

Figure 4.8: Pseudorapidity distribution of the highest- $p_{T}$ jet $\left(j_{1}\right)$ at NLO in $t$-channel production of a top quark at the Tevatron $(\sqrt{S}=2 \mathrm{TeV})$ after cuts. Phase space slicing (PSS) results (solid) and massive dipole formalism (MDF) results (dashed) are both shown.

order in QCD the cross sections factorize into two non-interfering production modes that may be identified by the $s$-channel or $t$-channel exchange of a $W$ boson. The $s$-channel cross section is characterized by having a typical final state of a top quark and a bottom-quark jet. The jet in the $t$-channel cross section tends to be somewhat more forward, and rarely contains a bottom quark. New physics scenarios tend to affect these production modes differently, and hence an accurate measurement and theoretical calculation of both modes is desirable. Furthermore, the cross section is directly proportional to the CKM matrix element $\left|V_{t b}\right|^{2}$, thus the ability to extract $V_{t b}$ is ultimately limited by our ability to predict the measured exclusive cross sections with experimental cuts.

The total cross sections for $s$ - and $t$-channel production are updated with CTEQ 5 parton distribution functions for runs I and II of the Tevatron, and for the LHC. The $t$-channel cross section is smaller than previously published by $13 \%$. This decrease is due entirely to the correction of a bug in the parton distribution functions. As the Tevatron will continue to run at $1.96 \mathrm{TeV}$ instead of $2 \mathrm{TeV}$, the $s(t)$-channel production modes will produce $4 \%(7 \%)$ fewer events than previously expected. We discussed some subtleties in estimating the effects of higher-order corrections on these cross sections.

We showed that the phase space slicing methods of one and two cutoffs and the massive dipole formalism produce the same jet distributions and cross sections. The 
dipole calculation retains the full spin dependence of the external particles, and so may be used to predict spin-dependent correlations of the partons.

This completes the first half of this thesis. We have computed the fully differential cross section for single-top production, which will be very important when precision measurements of this process become available. Due to the limited amount of data available for this thesis, we will now switch our attention to top pair production. 


\section{Chapter 5}

\section{Top pair production}

So far we have concentrated on developing a theoretical description of single-top production, up to next to leading order accuracy. Obviously, we would like to compare the theoretical prediction to experiment. However, the amount of Tevatron data available at the time of writing of this thesis was too low to make this possible. As mentioned in the introduction, the measurements of the single-top and $t \bar{t}$ processes are closely related. Thus, in the second part of this thesis we will describe a measurement of the top pair (also called $t \bar{t}$ ) cross section at the DØ experiment at Fermilab. We will start with a description of the measurements and theoretical calculations that have been done on top pair production.

The top quark was discovered at Fermilab's Tevatron collider, which during that time was colliding protons and antiprotons at a center of mass energy of $1.8 \mathrm{TeV}$. The first evidence for $t \bar{t}$ production was claimed by the CDF collaboration in April of 1994 [98]. The discovery of the top quark was made the following year by both the CDF and D $\varnothing$ collaborations. CDF reported a $t \bar{t}$ production cross section of $\sigma_{t \bar{t}}=6.8_{-2.4}^{+3.6 \mathrm{pb}}$ for a top mass $^{1}$ of $176 \mathrm{GeV}$ [99], and $\mathrm{D} \varnothing$ found $\sigma_{t \bar{t}}=6.4 \pm 2.2 \mathrm{pb}$ for a top mass of $199 \mathrm{GeV}[100]$.

In 2001, the CDF collaboration reported $\sigma_{t \bar{t}}=6.5_{-1.4}^{+1.7} \mathrm{pb}$ for $m_{t}=175.0 \mathrm{GeV}$ [101] as their final $t \bar{t}$ production cross section based on the 1992-1996 run of the Tevatron. The corresponding D $\varnothing$ result (published in 2003) is $\sigma_{t \bar{t}}=5.7 \pm 1.6 \mathrm{pb}$ for $m_{t}=172.1$ $\mathrm{GeV}$ [102]. In addition, measurements of the top mass were made. Combining results from D $\varnothing$ and CDF, the measured top mass is $174.3 \pm 5.1 \mathrm{GeV}$ [103].

On the theoretical side, many calculations of the top pair production cross section have been performed. Recent calculations are at next-to-leading-order (NLO) [60]. Some representative Feynman diagrams for this calculation are shown in Figure 5.1.

\footnotetext{
${ }^{1}$ The measured cross section is always quoted together with the top mass used for the analysis. This is because the signal efficiency and thus the cross section measurement depends on the top mass. This mass is taken from a separate measurement, which we will not discuss here.
} 

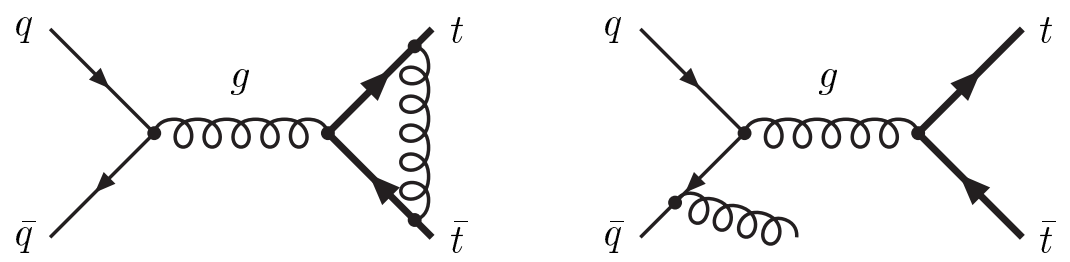

Figure 5.1: Some of the Feynman diagrams that contribute to the NLO cross section. The top quark line is thickened.

Beyond NLO, two techniques have been used. The first method [104, 105] constructs a double differential NNLO estimate. The cross sections found for a top mass of 175 $\mathrm{GeV}$ using this method are:

$$
\begin{aligned}
\sigma_{t \bar{t}}(1.8 \mathrm{TeV}) & =5.8 \pm 0.4 \pm 0.1 \mathrm{pb} \\
\sigma_{t \bar{t}}(1.96 \mathrm{TeV}) & =7.4 \pm 0.5 \pm 0.1 \mathrm{pb}
\end{aligned}
$$

The first uncertainty indicates an ambiguity in the kinematics, while the second is an estimate of the scale uncertainty. It should be noted that the uncertainty due to the parton distribution functions is not included.

In the second technique, the full next to leading log (NLL) resummation is computed $[106,107]$ for the inclusive cross section. This method gives the following cross sections for a top mass of $175 \mathrm{GeV}$ :

$$
\begin{aligned}
\sigma_{t \bar{t}}(1.8 \mathrm{TeV}) & =5.19_{-0.68}^{+0.52} \mathrm{pb} \\
\sigma_{t \bar{t}}(1.96 \mathrm{TeV}) & =6.70_{-0.88}^{+0.71} \mathrm{pb} .
\end{aligned}
$$

All theoretical uncertainties are included in these results.

Although these very advanced NLO differential and even higher order inclusive cross section calculations are available, until recently events generated for the full detector simulation used only LO accuracy for the top pair production process. This was due to problems in matching the beyond leading order calculations with the showering required for full event generation. These issues have now been solved, and an event generator has recently become available that treats the top pair production process to NLO accuracy (MC@NLO) [108].

We show some results for this MC@NLO program for a proton-antiproton collider at a center of mass energy of $1.96 \mathrm{TeV}$. In Figure 5.2(a) we compare the differential cross section as a function of the transverse momentum of the top quark that we get from MC@NLO with the LO HERWIG [14] result. In Figure 5.2(b) we do the same for the transverse momentum of the $t \bar{t}$ pair. At the high end of the second distribution, 


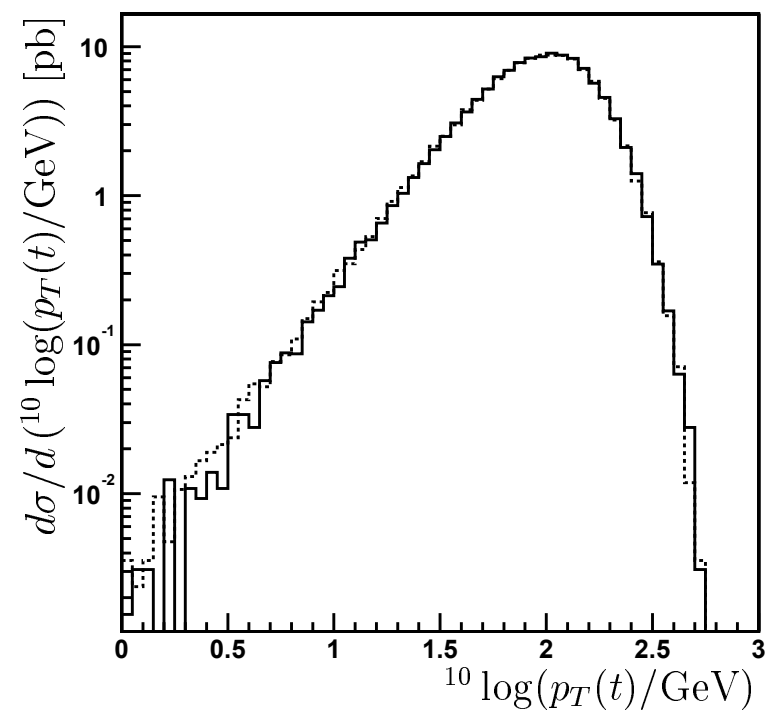

(a)

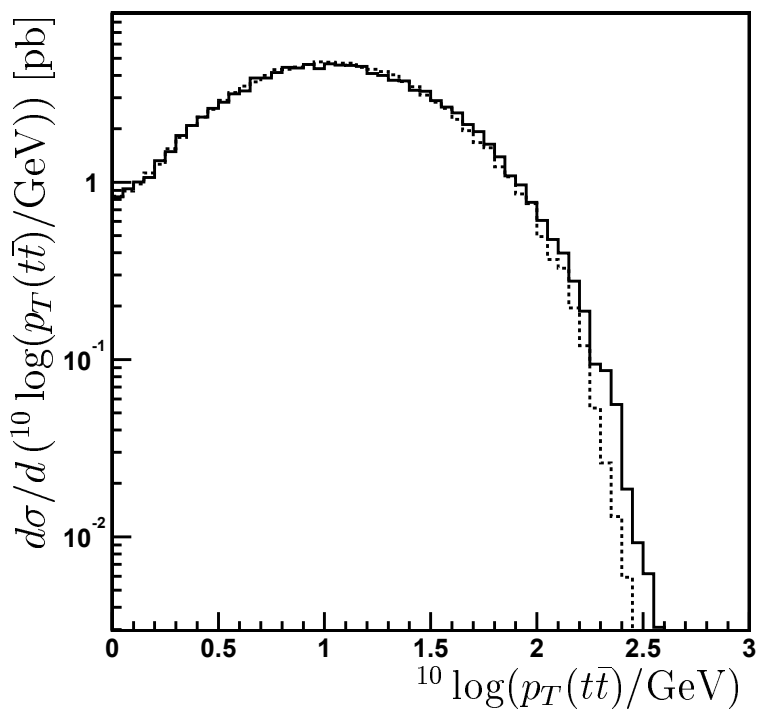

(b)

Figure 5.2: Differential cross section as a function of the transverse momentum of the top quark (a) and the transverse momentum of the $t \bar{t}$ pair (b). The solid line is the NLO result from MC@NLO, the dashed line is the LO result from HERWIG. The total $L O$ cross section is scaled to the NLO result.

we see that the NLO prediction is higher than the LO prediction. This is because hard emissions are treated correctly in the former.

After this short discussion of previous measurements and theoretical calculations, we shall describe a measurement of the top pair cross section. In Chapter 6 we will discuss the current state of the collider, the experiment and the trigger used for the analysis, followed by a chapter on the identification of physics objects (like electrons and jets). Finally, in Chapter 8 the actual analysis will be described. 


\section{Chapter 6}

\section{Experimental setup}

\subsection{The Tevatron collider}

The Tevatron collider is a proton-antiproton collider situated at Fermilab, near Chicago. The protons and antiprotons are produced and accelerated in a chain of accelerators before being injected into the Tevatron, which is depicted in Figure 6.1. The first step in the chain is the Cockroft-Walton accelerator, where negatively charged hydrogen ions are accelerated to $750 \mathrm{keV}$. Bunches of these hydrogen ions are accelerated up to $400 \mathrm{MeV}$ by a linear accelerator (the LINAC). The hydrogen ions are now stripped of their electrons by going through a carbon foil, and are then accelerated up to an energy of $8 \mathrm{GeV}$ by the booster, a circular accelerator. At this energy the protons are put into the Main Injector, which accelerates them either to $150 \mathrm{GeV}$ for injection into the Tevatron, or up to $120 \mathrm{GeV}$ for the production of antiprotons. In the later case, the $120 \mathrm{GeV}$ protons are collided with a nickel target, producing many secondary particles. About one in every $10^{5}$ protons produces an antiproton among the secondary particles. These antiprotons are collected and stored in the accumulator or antiproton source. When enough antiprotons have been collected, bunches of protons (at $150 \mathrm{GeV}$ ) are injected into the Tevatron by the Main Injector, followed by bunches of antiprotons from the accumulator, which are also accelerated up to $150 \mathrm{GeV}$ by the Main Injector. The Tevatron finally accelerates the bunches of protons and antiprotons up to $980 \mathrm{GeV}$. The beams are made to collide at two interaction points along the Tevatron, where the CDF and $\varnothing \varnothing$ experiments are located, resulting in $p \bar{p}$ collisions at a center of mass energy of $1.96 \mathrm{TeV}$.

\section{Luminosity}

An important collider parameter is the luminosity. The instantaneous luminosity is related to the number of particles that cross the collision region per unit of (transverse) 


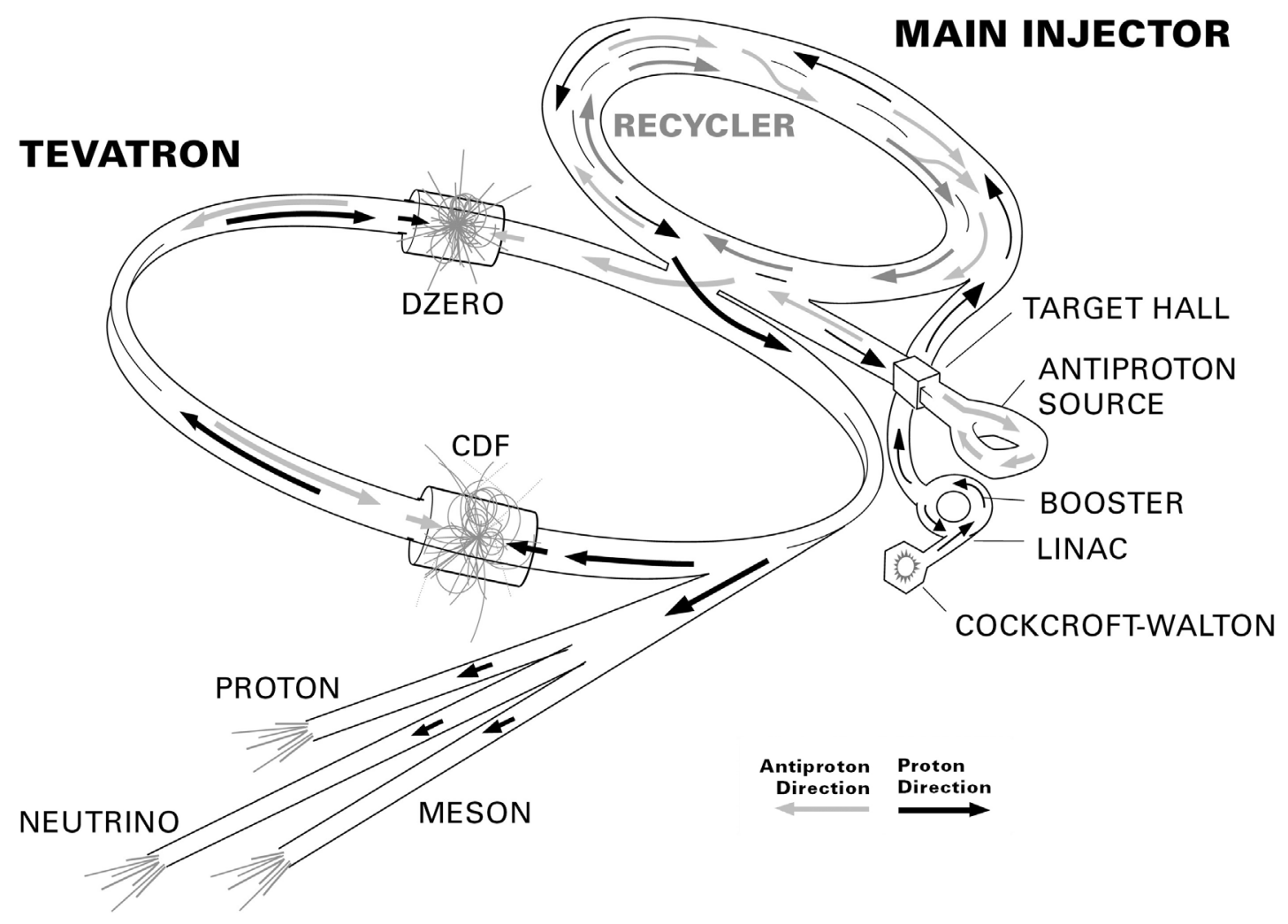

Figure 6.1: Fermilab accelerator complex.

area per second. The event rate $\frac{d N}{d t}$ of a certain physical process is proportional to the instantaneous luminosity $L$ :

$$
\frac{d N}{d t}=L \cdot \sigma_{\text {proc }} .
$$

This proportionality constant $\sigma_{\text {proc }}$ is called the cross section for that process. This can obviously be measured in an experiment, and for certain well defined processes, the cross section can also be calculated from theory. This makes the cross section a very interesting property of processes, because it can be used to compare theory and experiment. Integrating the previous equation, we see that the total number of events available is determined by the integrated luminosity:

$$
\int_{\Delta t} L \mathrm{~d} t=\frac{N}{\sigma_{\mathrm{proc}}} .
$$

The typical cross sections for processes that we are interested in are expressed in picobarn (pb, 1 barn $\equiv 10^{-28} \mathrm{~m}^{2}$ ), so the integrated luminosity is often quoted in units 
of inverse cross section, such as $\mathrm{pb}^{-1}$.

\subsection{The DØ detector}

The DØ detector is a general purpose detector which is optimized for the measurement of electron and jet energies. It consists of several subdetectors, which are positioned more or less symmetrically around the interaction point. Starting at the interaction point, moving outward, the $\mathrm{D} \varnothing$ subdetectors are:

- Silicon Microstrip Tracker (SMT);

- Central Fiber Tracker (CFT);

- Central and Forward Preshowers (CPS and FPS);

- Calorimeter;

- Muon system.

In addition to these subdetectors that cover more or less all directions, there are the Luminosity Monitors which cover only a small region. A cross section view of the detector is given in Figure 6.2.

The following convention for coordinates is used: The direction of the protons is the positive $z$ direction. The positive $x$ direction points away from the center of the Tevatron ring and thus the positive $y$ direction points up. In hadron colliders, pseudorapidity is normally used instead of the polar angle. The pseudorapidity $\eta$ is defined as $\eta=-\log \tan (\theta / 2)$, where $\theta$ is the polar angle (the angle with respect to the proton beam).

\subsubsection{Inner tracker}

Together, the SMT and the CFT form the inner tracker. They are placed in a 2.0 Tesla solenoidal magnet of about 1.2 meter diameter, which makes it possible to measure the transverse momentum and the charge-sign of charged particles. A cross section view of the inner tracker is given in Figure 6.3.

The SMT is composed of silicon strip detectors arranged in disks and cylinders centered on the beam. Most silicon modules used are double sided, so that all three coordinates of the hit position are measured. The number of hits on a typical track (at low $\eta$ ) is 4 .

The CFT is made of $830 \mu \mathrm{m}$ diameter scintillating fibers. They are arranged in 8 layers between radii 20 and $60 \mathrm{~cm}$, each layer containing a sublayer of axial fibers and a sublayer of stereo fibers mounted under a 3 degree angle. Due to the high efficiency 


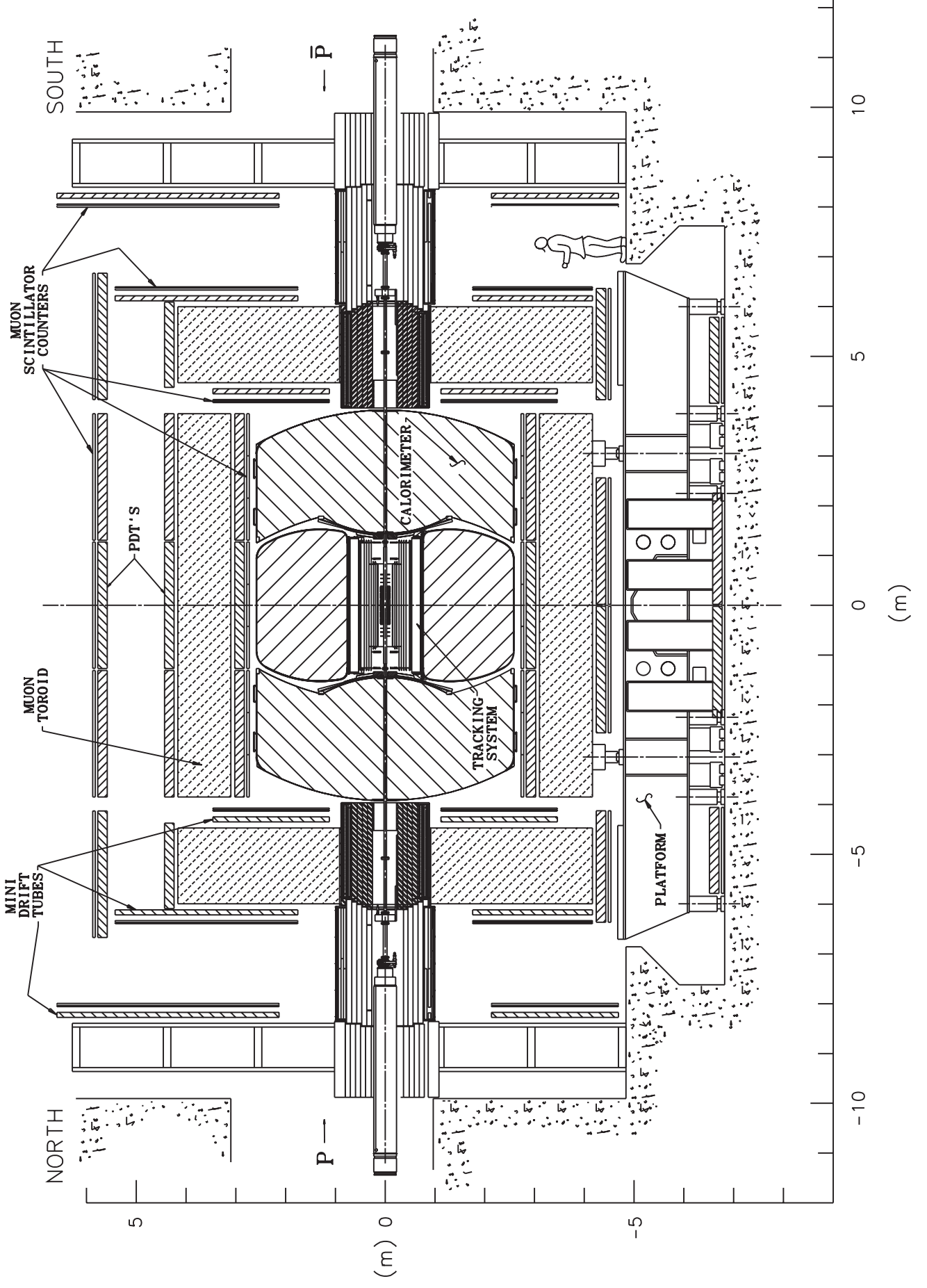

Figure 6.2: Cross sectional view of the $D \varnothing$ detector. 


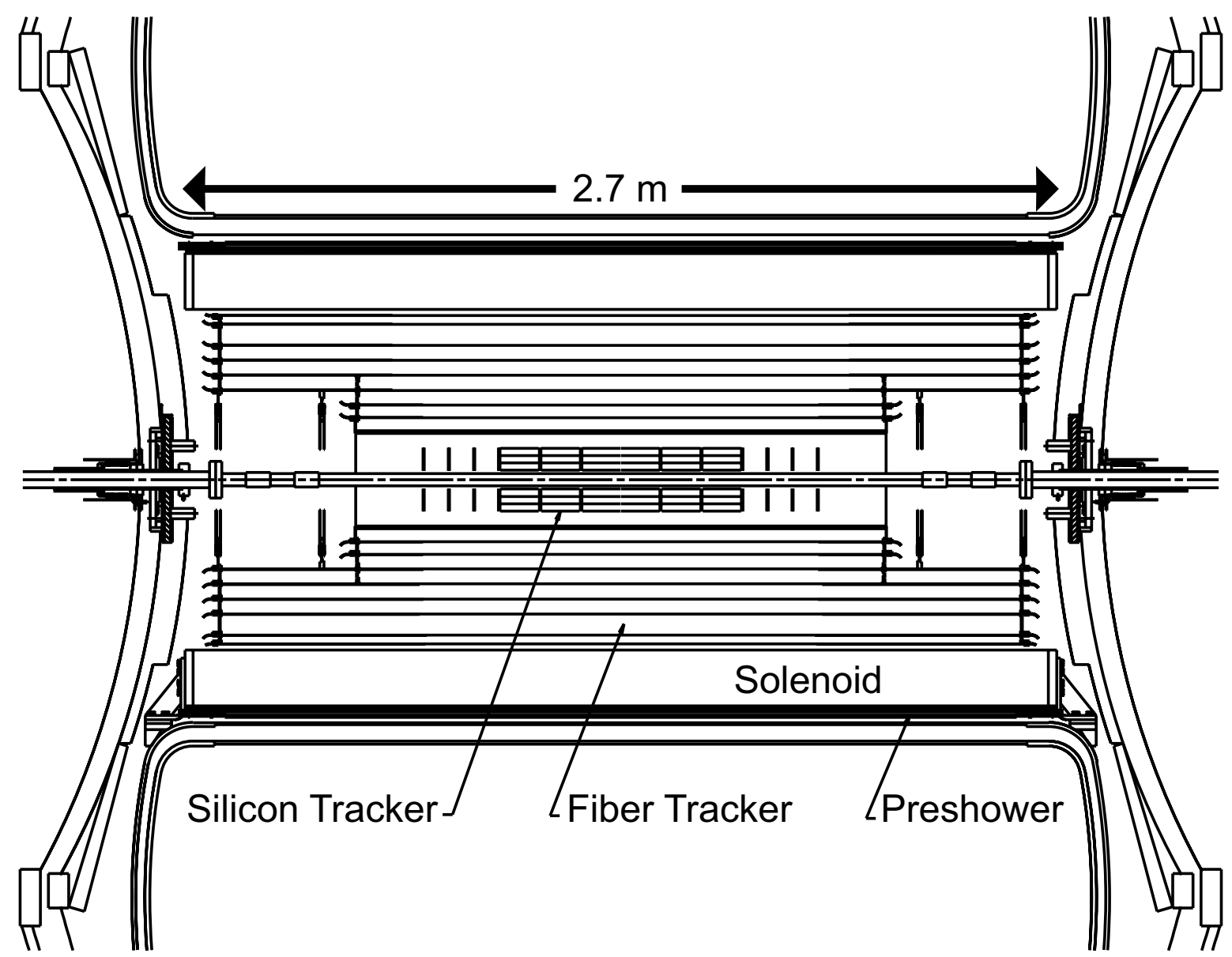

Figure 6.3: Schematic view of the inner tracker.

of the light collection, the typical number of measurements on a track should equal its maximum value of 16 ( 8 points).

\subsubsection{Calorimeter}

The $\mathrm{D} \varnothing$ calorimeter is a liquid argon calorimeter. It consists of three parts: the central calorimeter (CC) and two endcap calorimeters (EC). The CC covers the area up to $|\eta|$ of 1.2, and the EC extends the coverage in $|\eta|$ to above 4.0.

Each calorimeter is built up out of the inner electromagnetic (EM) layers, followed by the fine hadronic (FH) layers, and the coarse hadronic $(\mathrm{CH})$ layer furthest from the interaction point. The electromagnetic and fine hadronic layers use uranium absorber plates, while both copper and stainless steel is used in the coarse hadronic. Each layer is split into cells, which have a size of about $\Delta \eta \times \Delta \phi=0.1 \times 0.1$, except for the third EM layer which has double the granularity. This is the layer in the calorimeter where 
electrons are expected to deposit most of their energy. The improved segmentation here gives a better position measurement of the electrons. Beyond $|\eta|$ of 3.2 the overall cell size is increased to $\Delta \eta \times \Delta \phi=0.2 \times 0.2$, so that the physical size of these cells is still reasonable. The cells are arranged in towers. The arrangement of towers is projective, i.e. the towers point toward the interaction point, but the cells themselves are not. This can clearly be seen in Figure 6.4.

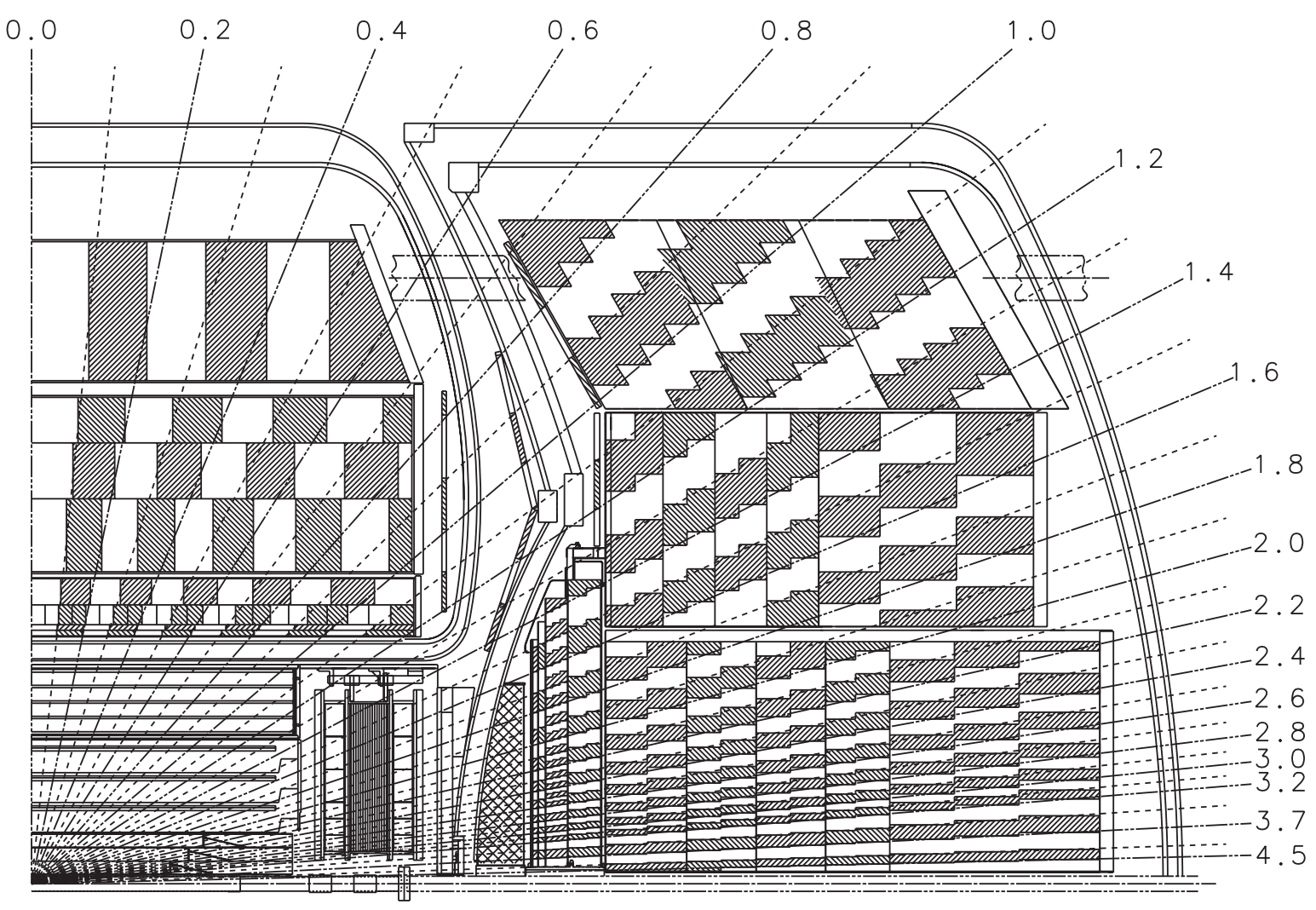

Figure 6.4: Side view of a quarter of the calorimeter. The lines with numbers at the end are lines of constant $\eta$. This is a picture of the Run I detector, so the depiction of the area inside and between the calorimeters is not correct for Run II.

In between the cryostats of the forward and central calorimeters scintillating material has been placed (covering $1.1<|\eta|<1.4$ ) to improve the energy measurement for particles traversing this area. This subdetector is called the intercryostat detector (ICD).

\subsubsection{Preshowers}

The central and forward preshower detectors are placed before the CC and EC calorimeters. They consist of lead radiators combined with scintillating material. These are 
intended to be used for particle identification. As these were not well understood at the time of this analysis, they were not used.

\subsubsection{Muon system}

The DØ muon system consists of several layers of drift chambers and scintillators, combined with a toroidal field solid-iron magnet. A detailed description can be found in [109]. Here it will suffice to say that the system can both identify and measure the momenta of muons out to $|\eta|$ of 2.0 .

\subsubsection{Luminosity Monitor}

The delivered luminosity (which is an important quantity, as we have seen) at $D \varnothing$ is measured by looking at the total event rate for $p \bar{p}$ inelastic interactions. This rate is measured by hodoscopes of scintillation counters mounted close to the beam on the inner surfaces of the endcap calorimeters. As the acceptance of the hodoscopes and the total cross section of $p \bar{p}$ inelastic interactions (measured by CDF, E710 and E811) are known, the instantaneous luminosity can be calculated (Eq. 6.1).

\subsection{Trigger}

\subsubsection{Trigger system}

For the analysis described in this thesis three different parts of the detector system are used for triggering purposes: the calorimeter, the muon system and the luminosity monitors.

\section{Overview}

The D0 Run II trigger system is designed to operate in a high rate environment, taking an input rate of $7 \mathrm{MHz}$ (132 ns bunch crossing time, at present the bunch crossing time is $396 \mathrm{~ns}$ ) and filtering out interesting physics events at the rate of $50 \mathrm{~Hz}$, without incurring more than $5 \%$ deadtime. The trigger system is organized in three major levels, the hardware based Level 1 (L1) and Level 2 (L2) and the software based Level 3 (L3). We will describe the state of the trigger as it was used to collect the data used in this analysis.

The hardware L1 trigger takes input from the calorimeter, the muon system and the Luminosity Monitor, and produces as output a list of trigger terms. The L1 Framework (L1FW), employing a series of Field Programmable Gate Arrays (FPGA's), examines these trigger terms and issues a L1 accept when certain criteria are met. 
The L2 trigger is comprised of two stages, a preprocessor stage and a global processor stage. In the preprocessors stage, each individual subdetector forms physics objects. In the global processor stage, physics objects from the different preprocessors can be combined to make the final L2 trigger decision.

Once a L2 accept has occurred, digitized data is loaded onto the single board computers (SBC) that sit in the front-end crates. Data from the SBCs are then transferred to the L3 farms where sophisticated filter codes are run to identify interesting events. At this level close-to-offline reconstruction of physics objects like electrons, muons, jets, missing $E_{T}$ is performed. Events satisfying L3 filter requirements are then transferred to tape for offline reconstruction.

\section{Calorimeter}

At L1, calorimeter triggering is based on towers. The size of these trigger towers is $0.2 \times 0.2$ in $\eta \times \phi$ space. Both the energy deposited in the EM layers of the tower and the total energy deposited in the tower (excluding the coarse hadronic layers) is available. Triggers can be set to require a number of both EM and total towers above certain thresholds in $E_{T}$. An example is $\operatorname{CEM}(1,10) \mathrm{CJT}(2,5)^{1}$. This requires an EM tower with $E_{T} \geq 10 \mathrm{GeV}$, and two total (jet) towers above $5 \mathrm{GeV}$. It should be noted that the EM and total towers are not exclusive, so the EM tower above $10 \mathrm{GeV}$ will also count as one of the total towers.

At L2, trigger decisions are also based on towers. L2 receives from L1 all trigger towers, both the EM $E_{T}$ and total $E_{T}$. L1 also sends two seed masks (one for EM and one for total), which have bits for every trigger tower. The EM bit in the seed mask is set to 1 if the trigger tower has at least $1 \mathrm{GeV}$ of EM $E_{T}$. The total bit is set to 1 if the trigger tower has at least $2 \mathrm{GeV}$ total $E_{T}$. The L2 calorimeter algorithms will only attempt to find jets or electrons around trigger towers for which the seed bit is set. Electromagnetic (EM) and jet objects are handled in different ways.

For every EM seed tower, the neighbor with the largest EM $E_{T}$ deposit is found, out of the 4 neighboring towers. The seed tower and its largest neighbor form the L2 electron. The total $E_{T}$ of the electron is the sum of the energy deposited in the EM layers of the two towers. The EM fraction of the L2 electron is the sum of the EM $E_{T}$ 's of the two trigger towers divided by the sum of the total $E_{T}$ 's of the two trigger towers. Trigger requirements can be placed on both the $E_{T}$ and the EM fraction of the L2 electrons.

For total (jet) seed towers all energy deposited in the $3 \times 3$ or $5 \times 5$ tower region $^{2}$ around the seed tower is added, and this is defined as a L2 jet. The $E_{T}$ of the L2 jet is the sum of the total transverse energy deposited in these 9 or 25 towers. Trigger

\footnotetext{
${ }^{1}$ The $\mathrm{C}$ in CEM and CJT indicates that this is a calorimeter trigger term.

${ }^{2}$ The algorithm was changed during the period that the data used in this analysis was taken.
} 
requirements are set on the number and $E_{T}$ of the L2 jets. Because of the algorithm used, all L2 electrons that pass a certain $E_{T}$ requirement will also pass as a L2 jet of the same $E_{T}$.

Both L1 and L2 use a readout of the calorimeter based on towers ("trigger readout") which is separate from the cell based readout ("precision readout") which is used for L3 and offline reconstruction.

At L3, the full cell information of the calorimeter is available. Again, L3 electrons and jets are treated differently. L3 electrons start out using a narrow cone (0.4) jet algorithm (based on towers). This defines the electron cluster. In the next step, only cells within a $\Delta R=\sqrt{\Delta \phi^{2}+\Delta \eta^{2}}$ of 0.25 around the axis of this cluster are used to define the electron object. Trigger requirements can be placed on the $E_{T}$ (which is the sum over all layers), EM fraction and width of the energy deposit in each of the EM layers. The width in EM layer $l$ is defined as

$$
W_{l}=\frac{\sum_{i} E_{i} \sqrt{\Delta \phi_{i}^{2}+\Delta \eta_{i}^{2}}}{\sum_{i} E_{i}},
$$

with i running over cells of the cluster in the $l^{\text {th }}$ EM layer, and $\Delta \phi$ and $\Delta \eta$ the distance (in $\phi$ and $\eta$ ) between the cell and the cluster axis.

L3 jets are found by using a cone algorithm (based on towers) with a cone size of 0.7. No splitting and merging of jets is performed. Again, because of the algorithms used, every electron above a certain $E_{T}$ threshold will also show up as a jet above that same threshold.

\section{Muon}

At L1, the trigger looks for hit coincidences between different layers of the muon scintillators and drift chambers. A coincidence with hits consistent with a $p \bar{p}$ interaction in the Luminosity Monitor can also be required. At L2, the drift chamber information is combined with the scintillator hits to do a full reconstruction of the muon. The same thing is basically done in the L3 trigger, only with higher precision.

For this analysis, muon based triggers were only used to define samples (for trigger studies) that are unbiased with respect to calorimeter triggers.

\subsubsection{Signal trigger}

The $t \bar{t}$ events that we are interested in for this analysis have the following event signature (see Section 8.1): one isolated electron and four or more jets. The signal trigger used to select these events relies solely on calorimeter information at all three levels of the trigger system. In designing this trigger, we have noted two important points: the single electron trigger rate is very high and runs the risk of being prescaled at high 
luminosity running, and we want to maximize efficiency by avoiding an overly stringent selection on the electron. We also note that, since there are several jets in signal events, it is efficient to require at least one jet. Therefore, at all three trigger levels, we select one moderate $p_{T}$ electron and one jet.

The used trigger is called EM15_2JT15, and the requirements at each level of the trigger are $^{3}$ :

- L1:

- one L1 electron with $E_{T} \geq 10 \mathrm{GeV}(\operatorname{CEM}(1,10))$

- one L1 jet with $E_{T} \geq 5 \mathrm{GeV}$ ( CJT $\left.(1,5)\right)$;

- L2:

- one L2 electron with $E_{T} \geq 10 \mathrm{GeV}, \mathrm{EM}$ fraction $\geq 0.85(\operatorname{L2} \operatorname{EM}(0.85,10))$

- one L2 jet with $E_{T} \geq 10 \mathrm{GeV}$;

- L3:

- one L3 electron with $E_{T} \geq 15 \mathrm{GeV}$, EM fraction $\geq 0.90$ and shower shape cuts of $W_{1}<0.09, W_{2}<0.08$ and $W_{3}<0.05 \quad($ L3Ele $(\mathrm{SH}, 15))$

- one L3 jet with $E_{T} \geq 15 \mathrm{GeV}$.

\subsubsection{Single object trigger efficiencies}

Trigger efficiencies are measured from data. To obtain an event efficiency in this situation for signal events, we measure the per-electron and per-jet efficiencies and fold them into the signal simulation to measure a probability that an individual event would fire the trigger.

We measure efficiencies for single objects with respect to $p_{T}$ and $\eta$ for each trigger term. An independent control sample, unbiased with respect to the trigger under test ("test trigger"), is defined. In such an unbiased sample, offline objects are identified, yielding the unbiased reference sample with a certain $p_{T^{-}}$or $\eta$-spectrum. The fraction of these events, for which the test trigger has fired, is a measure of the trigger efficiency with respect to the offline object $p_{T}$ or $\eta$. The resulting dependence of the efficiency versus $p_{T}$ is also termed 'turn-on curve'.

\footnotetext{
${ }^{3}$ It has been noted that at all levels of the trigger, electrons above a certain $p_{T}$ threshold also show up as a jet above that same threshold. We will not list those objects here. When one jet is required, we mean one jet in addition to the electron.
} 


\section{EM objects}

The electron trigger efficiencies are measured on events which were triggered by the muon system and which have an offline reconstructed electron. All offline electron energies are corrected with the EM energy corrections.

The L1 turn-on curve for $\operatorname{CEM}(1,10)$ is shown in Figure 6.5(a). The trigger efficiency is not $50 \%$ at $10 \mathrm{GeV}$, indicating that the gain of the L1 energy readout is low. The trigger reaches saturation at approximately twice the threshold. Figure 6.5(b) shows the efficiency as a function of detector ${ }^{4} \eta$ for electrons that have $p_{T}>20 \mathrm{GeV}$. There is low statistics around $\left|\eta_{D}\right|=1.3$ because hardly any offline electrons are reconstructed in this region. The EM layers don't have complete coverage in this ICD region, resulting in very low efficiency for electrons to pass the offline EM fraction requirement.

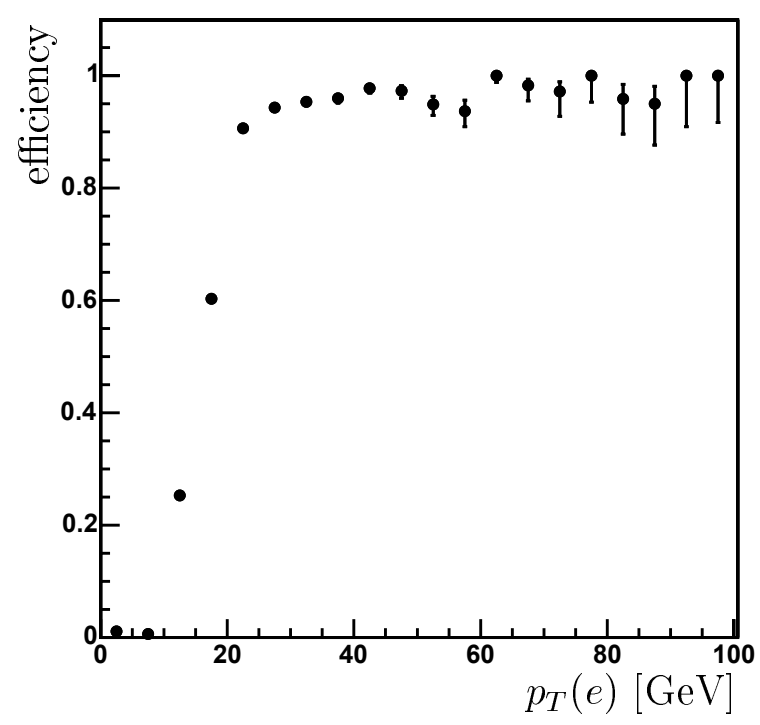

(a)

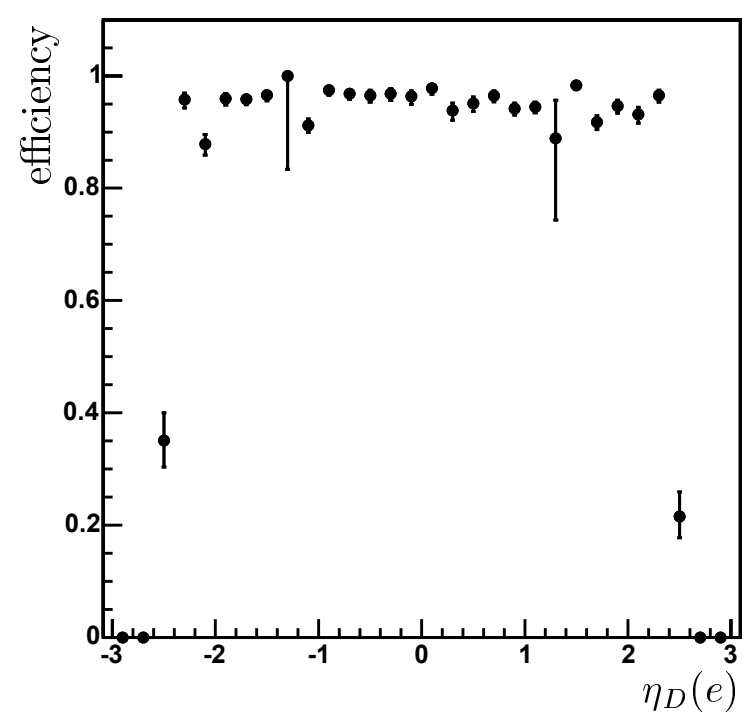

(b)

Figure 6.5: Efficiency for an electron to fire the CEM(1,10) trigger term, as a function of offline electron $p_{T}(\mathrm{a})$ and detector $\eta(b)$.

The Level 2 electron efficiency for $\operatorname{L} 2 \operatorname{EM}(0.85,10)$ is $100 \%$ for electrons with an offline $p_{T}>10 \mathrm{GeV}$ that have already passed L1 CEM $(1,10)$.

\footnotetext{
${ }^{4}$ Detector $\eta\left(\eta_{D}\right)$ is the $\eta$ calculated from the electron cluster position (see Chapter 7 ) and $(0,0,0)$. Detector $\eta$ is thus directly correlated with the position of the cluster in the calorimeter. This is different from the usual $\eta$ (also called physics $\eta$ ), which is taken from the direction of the track that has been associated with the electron.
} 
The Level 3 electron efficiency for L3Ele(SH,15) is measured on the Mark\&Pass events for the EM15_2JT15 trigger. These are events that passed the L1 and L2 requirements of the trigger, but where no trigger requirements were made at L3. Thus this efficiency is measured on objects that have passed the L1 and L2 electron requirements, and look like electrons offline. In Figure 6.6(a) its turn-on curve is shown, for events that have already passed the L1 \& L2 requirements discussed above. Figure 6.6(b) shows its efficiency as a function of detector $\eta$ for electrons that have $p_{T}>20 \mathrm{GeV}$.

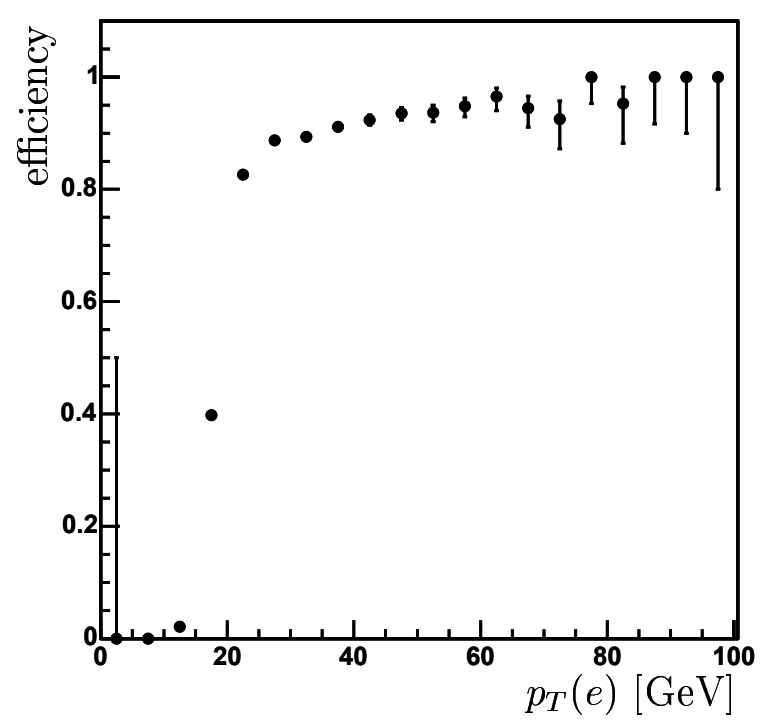

(a)

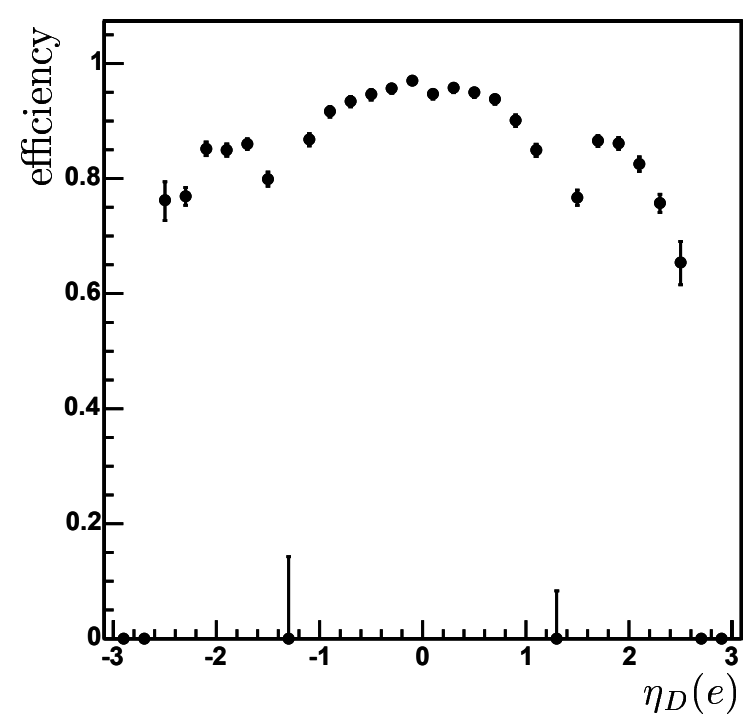

(b)

Figure 6.6: Efficiency for an electron to fire the L3Ele(SH,15) trigger term, as a function of offline electron $p_{T}$ (a) and detector $\eta(b)$. This is for electrons that have already passed the $L 1$ and L2 electron requirements.

\section{Jet objects}

The Level 1 efficiency for jets to fire the CJT(1,5) term is shown in Figure 6.7 as a function of the reconstructed jet $p_{T}$ and detector $\eta$. All offline jet energies and momenta are corrected with the Jet Energy Scale, which is described in Chapter 7. There are dips in the efficiency in the ICD region (around $\left|\eta_{D}\right|=1.1$ ) because the gain of the trigger readout is too low in this region. The efficiency falls off rapidly for $\left|\eta_{D}\right|>2.4$ because the trigger readout was not instrumented in that region.

The efficiency for a jet that has passed L1 CJT(1,5) to fire the L2 jet trigger with a threshold of $10 \mathrm{GeV}$ is shown in Figure 6.8(a) as a function of the reconstructed jet $p_{T}$. 


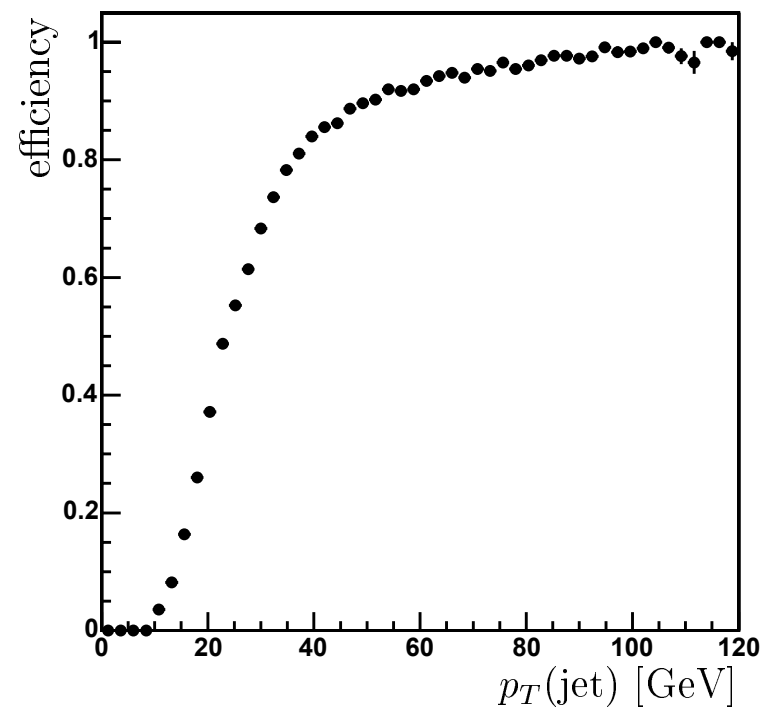

(a)

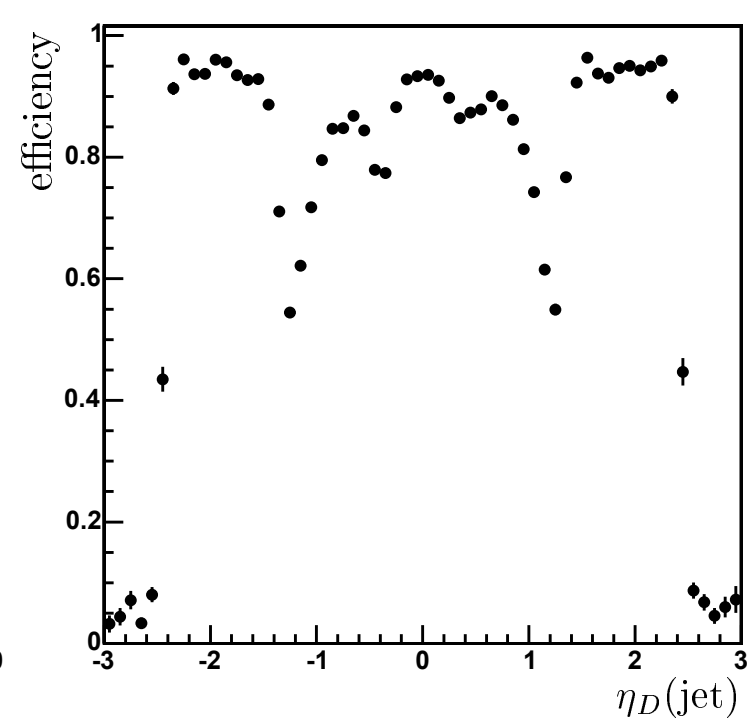

(b)

Figure 6.7: Efficiency for a jet to fire the CJT(1,5) trigger term, as a function of offline jet $p_{T}$ (a) and detector $\eta(b)$. The plot as a function of detector $\eta$ is for jets with an offline $p_{T}>30 \mathrm{GeV}$.

The Level 3 jet efficiency as a function of reconstructed jet $p_{T}$ is shown in Figure 6.8(b) for a Level 3 jet threshold of $15 \mathrm{GeV}$. This is for jets that have already passed L1 CJT $(1,5)$ and the L2 jet trigger at $10 \mathrm{GeV}$.

No parameterization was used for the jet turn-on curves. The full $\eta$ and $p_{T}$ dependent trigger curve is folded with the simulated $\mathrm{MC}$ jets distribution in order to obtain the jet part of the trigger efficiency for $t \bar{t}$ events.

\subsubsection{EM15_2JT15 efficiency}

For the analysis, we require that the electron is in the Central Calorimeter (see Chapter 8). Thus in this section where the final trigger efficiency is derived, trigger turn-on curves are used where the electron was required to be in the CC.

The turn-on curves for the EM and the jet terms for the three levels of trigger combined can be observed in Figures 6.9(a) and 6.9(b) respectively. For the electrons, we take only the $p_{T}$ dependence into account, because there is not enough statistics to use the full $\eta$ and $p_{T}$ dependence. It should be noted that the $\eta$ dependence is rather flat in the region used $\left(\left|\eta_{D}\right|<1.1\right)$. The parametrization used for the $p_{T}$ dependence is shown in the figure. For the jets, we take the full $\eta$ and $p_{T}$ dependence of the trigger 


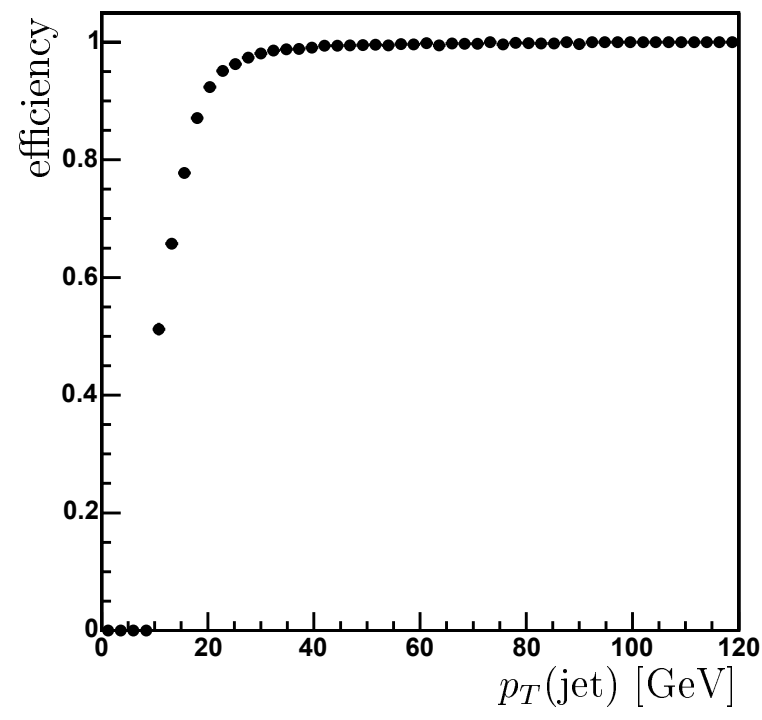

(a)

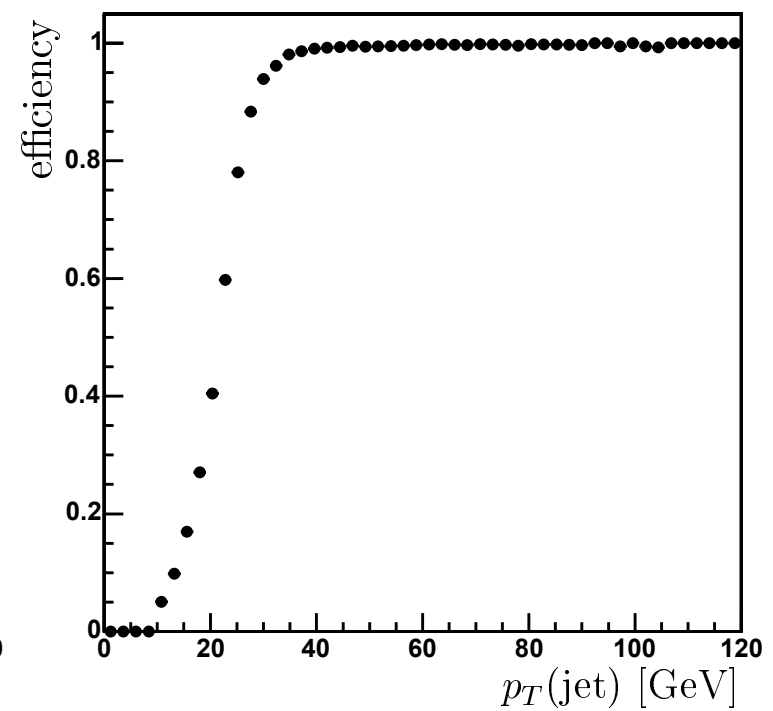

(b)

Figure 6.8: The efficiency for a jet to fire the L2 (a) and L3 (b) jet trigger, as a function of offline jet $p_{T}$.

efficiency into account, which is shown in Figure $6.10^{5}$. To obtain the trigger efficiency for the $t \bar{t}$ events, we assume that the trigger terms factorize:

$$
\varepsilon_{t \bar{t}}=\varepsilon_{\mathrm{EM}} \times\left[1-\prod_{\text {jets }}\left(1-\varepsilon_{\mathrm{jet}}\right)\right]
$$

When we fold the measured single object trigger efficiencies into the $t \bar{t}$ signal MC (using the above equation), we find a total trigger efficiency of $92 \pm 1 \%$.

The assumption of factorization could break down in the case where a jet fires part of the electron trigger. We studied the effect where the jet fires the L1 $\times$ L2 EM requirement, and the electron fires the L3 EM requirement. This could happen if the electron goes through a L1 trigger tower that is dead. By looking at the trigger turnon curve for jets to fire the EM trigger (see Figure 6.11) it was shown that at most $50 \%$ of the electron L1 $\times$ L 2 inefficiency can be recovered by this scenario. Therefore we will quote a $2 \%$ systematic uncertainty due to factorization. Thus the final trigger efficiency used is $92 \pm 1 \pm 2 \%$. The number given is the efficiency corresponding to those events passing all selection criteria and cuts.

\footnotetext{
${ }^{5}$ In this figure we can again see the low gain in the ICD region.
} 


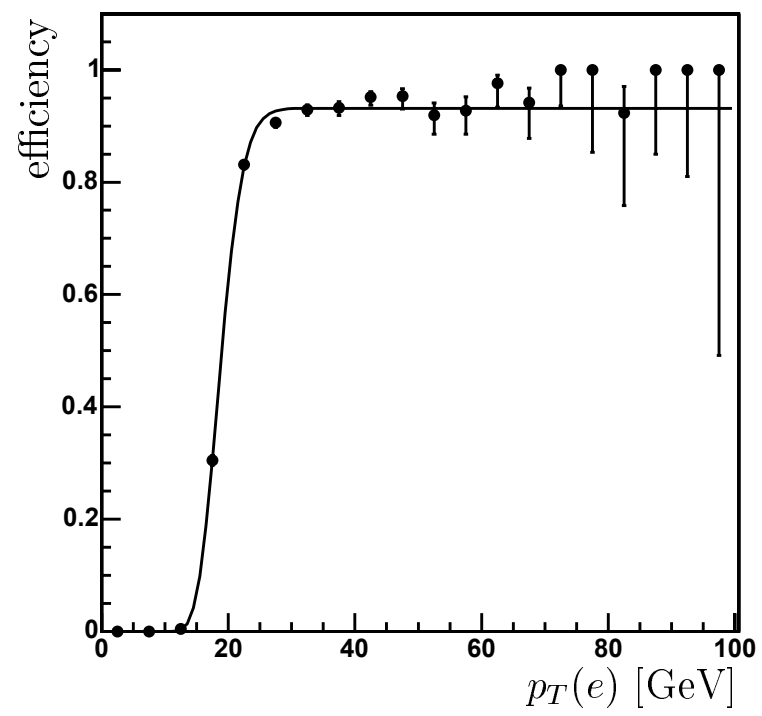

(a)

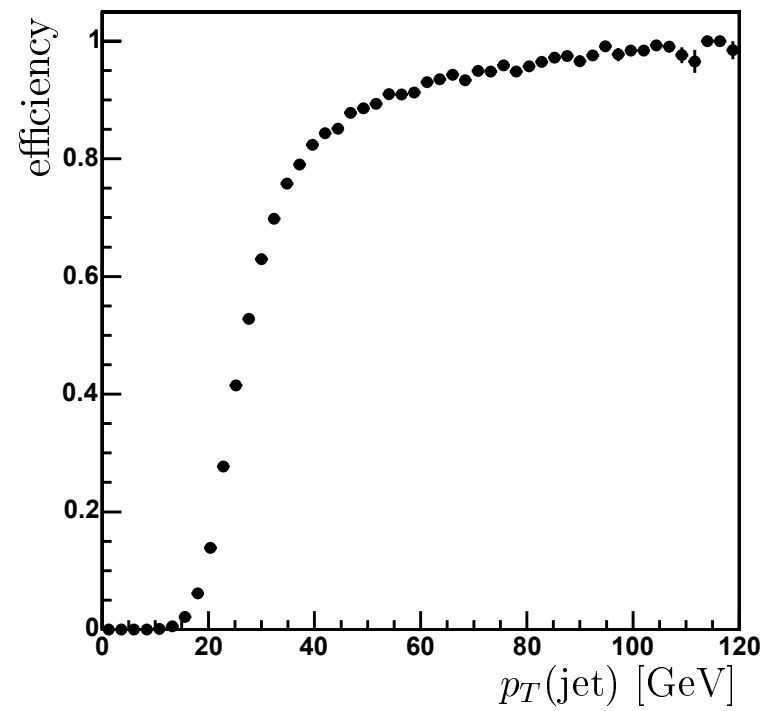

(b)

Figure 6.9: The combined $L 1 \times L 2 \times L 3$ trigger efficiency for electrons (a) and jets (b), as a function of offline $p_{T}$.

\subsubsection{Other triggers}

In addition to the EM15_2JT15 trigger other triggers were used. Examples of these are a single electron trigger, which was used in the analysis described in Chapter 8 to estimate the bias on the number of jets in an event introduced by requiring a jet in the trigger, and a two electron trigger used to define $Z \rightarrow e e$ samples for electron identification studies described in Chapter 7. Other examples are the muon based triggers, which were used to select unbiased samples for calorimeter trigger studies. The efficiency for these triggers was not derived, because the studies that they were used for do not depend on the trigger efficiency.

\subsection{Data processing}

After having passed the L3 trigger, events are written to tape in the raw data format. These events are later reconstructed on a dedicated farm of computers by the D $\varnothing$ reconstruction program ( $\mathrm{d} 0 \mathrm{reco})$. This program takes the raw data from the detector and reconstructs physics objects (electrons, tracks, jets), writing out these objects into a mini-DST, the so-called Thumbnail format. The next step is to select interesting events from this full set of events, based on simple requirements on the physics objects 


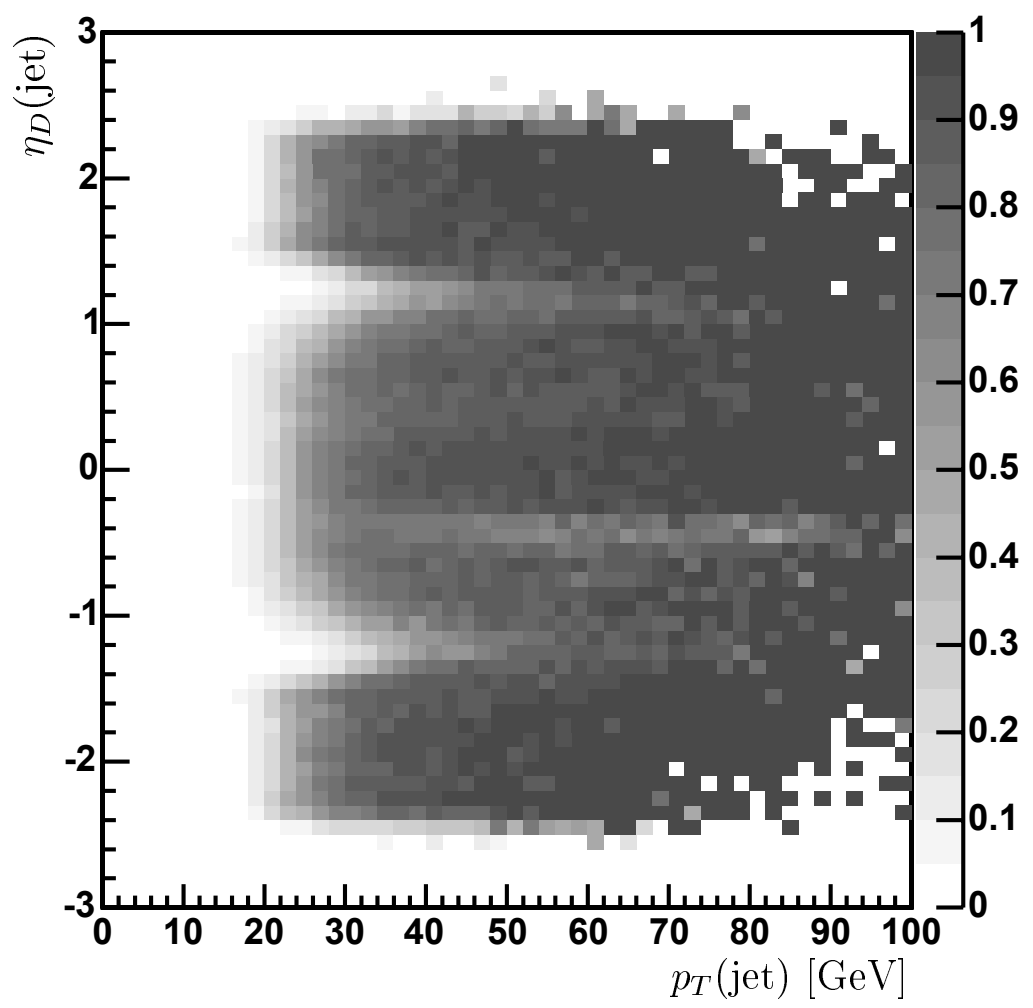

Figure 6.10: The combined $L 1 \times L 2 \times L 3$ jet trigger efficiency as a function of offline $p_{T}$ and $\eta$.

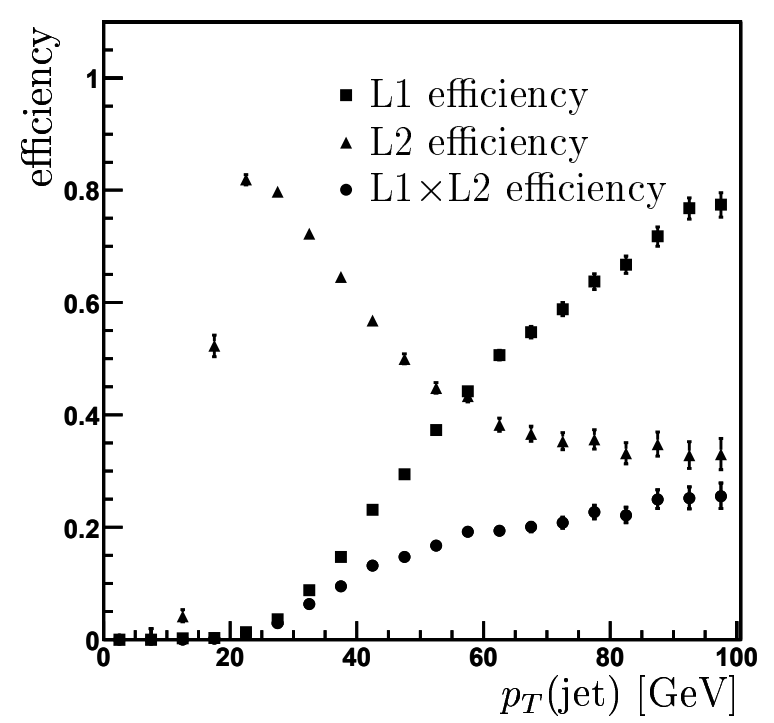

Figure 6.11: The efficiency for jets to fire the L1, L2 and L1×L2 EM trigger as a function of offline jet $p_{T}$. The shape of the L2 efficiency is due to the EM fraction cut at $L 2$. 
or by requiring that the events passed a certain trigger. This process is called skimming. The skimmed samples (which are still in Thumbnail format) are now processed through a program called top_analyze, which applies all the required energy corrections to the objects (see the next chapter), applies most of the object identification criteria, and does an even tighter selection of events. For the events selected in the top_analyze stage, a root tuple is written out containing all the information about the physics objects in the event. The final stage is a set of root macros that extracts the results from these root tuples.

\subsection{Signal and background simulation}

Apart from the actual data events, we also need simulated events. These events are used for estimating acceptances and certain efficiencies.

We simulated the $t \bar{t}$ signal and the $\mathrm{W}+$ jets background processes using ALPGEN 1.1 [110] for calculating the matrix elements, and PYTHIA [13] to perform the parton showering and hadronization of the ALPGEN partons. PYTHIA was also used to generate simulated $Z \rightarrow e e$ events. The output of PYTHIA is then processed through d0gstar (a GEANT simulation of the D $\varnothing$ detector). This step propagates the PYTHIA particles through a computer model of the detector, calculating how much energy the particles deposit in the material they traverse.

The next step in the simulation, d0sim, models the response of active material in the detector to these energy deposits. It also models the electronics that reads out these responses. In addition, it adds minimum bias events to the events. This models the fact that during any bunch crossing, except for the hard collision, there might also be one or more soft proton-antiproton collisions. The number of added minimum bias events is taken from a Poisson distribution with a mean of 0.8 events, which corresponds to the rate expected for the luminosity at which the data used for the analysis in this thesis was taken. Finally, d0sim writes out a raw data format that is equivalent to the raw data format that comes from the detector. These simulated events can thus now be processed exactly the same as real data events.

Simulated events that are produced in this way are called Monte Carlo (MC) events. 


\section{Chapter 7}

\section{Object identification}

In this chapter we will discuss how the different physics objects used in this analysis (electrons, jets and neutrinos) are identified in the detector.

\subsection{Electrons}

\subsubsection{Electron reconstruction and variables}

Electrons are reconstructed from information from the calorimeter and tracking subdetectors. This typically proceeds in two stages; a cluster is formed in the calorimeter, and subsequently confirmation is sought from the tracker.

\section{Calorimeter reconstruction}

At the reconstruction stage, an EM cluster is defined as a set of towers in a cone of radius $R=\sqrt{\Delta \eta^{2}+\Delta \varphi^{2}}=0.4$ which have a minimum transverse energy of 1.5 $\mathrm{GeV}$. Among all reconstructed clusters, genuine EM showers produced by electrons or photons are expected to have a large EM fraction $f_{\mathrm{EM}} \equiv E_{\mathrm{EM}} / E_{\mathrm{tot}}$, where $E_{\mathrm{EM}}$ is the cluster energy in the EM section of the calorimeter and $E_{\text {tot }}$ is its total energy within the cone (the EM fraction of a cluster can be greater than one because of the noise subtraction used on the calorimeter cells). In addition, genuine EM showers should have a longitudinal and lateral development compatible with that of an electron. Each cluster is attributed a $\chi_{\text {Cal }}^{2}$ (also called 'H matrix') based on the comparison of the energy deposited in each layer of the EM calorimeter with average distributions obtained from Monte Carlo simulation ${ }^{1}$.

\footnotetext{
${ }^{1}$ The variables used in the calculation of $\chi_{C a l}^{2}$ are: energy fractions in EM1, EM2, EM3 and EM4, two cluster sizes in EM3 (one in the $\phi$ direction, and the other in the direction perpendicular to $\phi$ in the EM3 plane), ${ }^{10} \log E$, and $z_{\text {vertex }}(e) / z_{w}$, where $z_{w}=28 \mathrm{~cm}$ is the $z$ width of the vertex distribution.
} 
Electron candidates are selected by requiring that

$$
\left.f_{\mathrm{EM}}>0.9 \text { and } \quad \chi_{\text {Cal }}^{2}<20 \text { (8 d.o.f. }\right) \text {. }
$$

We additionally require that the cluster is isolated:

$$
f_{\text {iso }}=\frac{E_{\mathrm{tot}}(R<0.4)-E_{\mathrm{EM}}(R<0.2)}{E_{\mathrm{EM}}(R<0.2)}<0.15 .
$$

\section{Central track confirmation}

The electron reconstruction in the calorimeter suppresses a large portion of the QCD background contamination. However, due to the overwhelming nature of this background, the additional rejection of a matching central track has typically been required. This also rejects photons, in which we are not interested for this analysis.

For each EM cluster candidate and each available track, a $\chi^{2}$, defined as

$$
\chi_{E M-\text { track }}^{2}=\left(\frac{\delta \varphi}{\sigma_{\varphi}}\right)^{2}+\left(\frac{\delta z}{\sigma_{z}}\right)^{2}+\left(\frac{E_{T} / p_{T}-1}{\sigma_{E / p}}\right)^{2},
$$

is computed. In these expressions:

- $\delta \varphi($ resp. $\delta z)$ denotes the difference between $\varphi$ (resp. $z$ ) of the track impact at the third EM layer and $\varphi$ (resp. $z$ ) of the center of gravity of the cluster position measured therein;

- $E_{T} / p_{T}$ is the ratio of the measured transverse energy of the cluster to the measured $p_{T}$ of the track;

- $\sigma_{\varphi}, \sigma_{z}$ and $\sigma_{E / p}$ are the root-mean-square (RMS) of the experimental distributions of the three associated quantities $\left(\delta \varphi, \delta z\right.$ and $\left.E_{T} / p_{T}-1\right)$.

A cluster is matched when the condition $\operatorname{Prob}\left(\chi_{E M-\text { track }}^{2}, N_{\text {dof }}\right)>1 \%$ is met by at least one track. If more than one track meets this condition, the one with the highest $\operatorname{Prob}\left(\chi_{E M-\text { track }}^{2}, N_{\text {dof }}\right)$ is selected.

\section{Likelihood confirmation}

The confirmation of an electron through the presence of a central track is a powerful technique. However, in seeking to reduce the background further one may take advantage of further pieces of available information.

For this analysis, we will replace the step of track confirmation with a likelihoodbased confirmation which has the advantage of taking into account more pieces of 
information, and which provides characterization of both the background and the signal shapes.

Further, we will extract the discrimination in the $E_{T} / p_{T}$ variable directly as part of the likelihood, rather than including it in the EM-track match $\chi^{2}$ quantity. This frees us from assuming that the distribution is Gaussian, and allows us to take advantage of our knowledge of the shape of the background as well. Thus, the likelihood will include six quantities:

- $\log \left(\operatorname{Prob}\left(\chi_{\text {spatial }}^{2}\right)\right)$, where

$$
\chi_{\text {spatial }}^{2}=\left(\frac{\delta \varphi}{\sigma_{\varphi}}\right)^{2}+\left(\frac{\delta z}{\sigma_{z}}\right)^{2}
$$

- $E_{T} / p_{T}$, described above;

- H matrix or $\chi_{\text {Cal }}^{2}$, described above;

- EM fraction, or $f_{\mathrm{EM}}$, described above;

- Distance of Closest Approach (DCA) measures the shortest distance of the selected track to the line parallel to the $z$ axis which passes through the primary vertex position;

- Track isolation $\left(\Delta R_{2}\right)$ measures the distance in $R$ to the track second closest to the EM cluster, with the assumption that the closest track is likely to be due to the passage of the electron.

\subsubsection{Sources of background}

As discussed in [111], the predominant sources of background to reconstructed electrons are $\pi^{0}$ showers with an overlapping track from a charged particle and photons converting to $e^{+} e^{-}$pairs. Without a measurement of the energy loss, $d E / d x$, it is difficult to separate these two components of the background ${ }^{2}$.

In terms of the variables defined above, photon conversions may be marked by the presence of a second track extremely close to the EM cluster, and a large $E_{T} / p_{T}$; their calorimeter quantities, however, would be nearly identical to that of an electron. Asymmetric conversions would give a nearly irreducible background as the second electron may be very soft.

\footnotetext{
${ }^{2}$ It should be possible to study the behavior of both components separately by looking at Monte Carlo events. In practice, this is hard due to the very small probability of having a fake electron in an event.
} 
Neutral pions may also have nearby tracks, as they are produced in association with other charged hadrons; if the track and the EM cluster arise from separate objects then their $E_{T} / p_{T}$ would not tend to be one and their track matching would be poor. Their $\chi_{\text {Cal }}^{2}$ and $f_{\mathrm{EM}}$ may be worse due to the surrounding hadrons.

\subsubsection{Likelihood formulation}

We begin with an empirical description of typical distributions

$$
P(\bar{x} \mid \operatorname{sig}), P(\bar{x} \mid b k g)
$$

in a vector of variables $\bar{x}$ for signal and background objects, respectively. Assuming flat prior distributions, we can describe

$$
P_{s i g}(\bar{x}), P_{b k g}(\bar{x}) \text {. }
$$

We further make the simplifying assumption that $P$ is uncorrelated in $\bar{x}$ (see Figure 7.1) so that

$$
P(\bar{x})=\prod_{i} P\left(x_{i}\right) .
$$

To select between objects that are likely to be signal and those that are likely to be background, we form a simple discriminant:

$$
D(\bar{x})=\frac{P_{s i g}(\bar{x})}{P_{s i g}(\bar{x})+P_{b k g}(\bar{x})}
$$

\subsubsection{Likelihood performance}

\section{Dataset definitions}

We construct two typical samples, one enriched in electrons and the other in background. We do this for both the central $\left(\left|\eta_{D}\right|<1.1\right)$ and endcap $\left(1.5<\left|\eta_{D}\right|<2.5\right)$ calorimeters and treat these separately. The region in between $\left(1.1<\left|\eta_{D}\right|<1.5\right)$ is not used, because the coverage of the EM layers is incomplete there, resulting in very few electron candidates in this region.

The electron sample is selected from $Z \rightarrow e e$ events, requiring:

- 2 EM clusters, each with:

$$
\begin{aligned}
& -p_{T}>20 \mathrm{GeV} \\
& -\chi_{\text {Cal }}^{2}<20, f_{\mathrm{EM}}>0.9, f_{\text {iso }}<0.15
\end{aligned}
$$



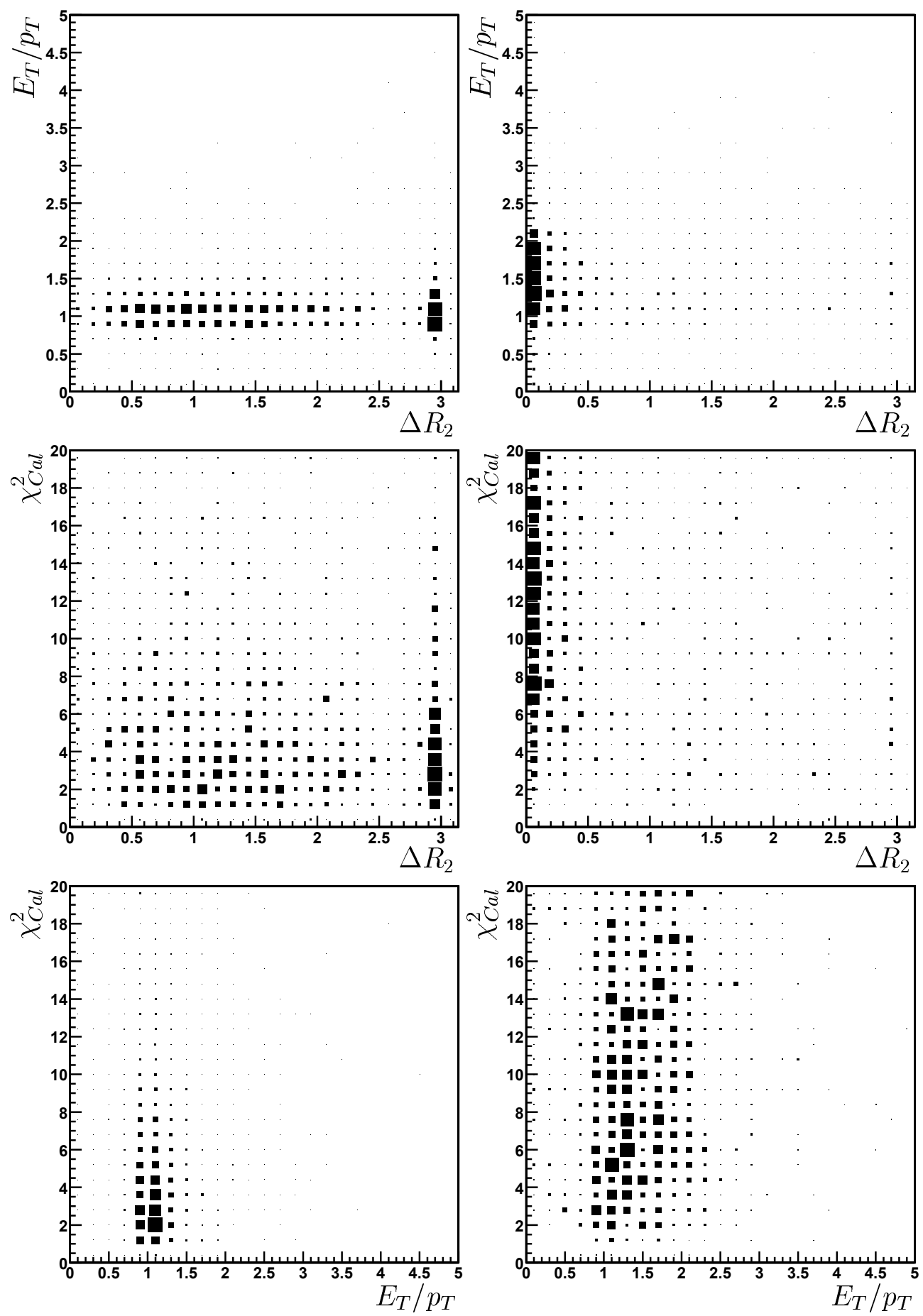

Figure 7.1: Correlations between selected variables used in the construction of the likelihood, for the central calorimeter signal (left) and background sample (right). Distributions are shown only for those electrons which have a track candidate (as defined in the text). 


\begin{tabular}{l|r|r}
\hline \hline Sample & CC & EC \\
\hline \hline Signal & $77 \%$ & $63 \%$ \\
\hline Background & $6 \%$ & $15 \%$ \\
\hline \hline
\end{tabular}

Table 7.1: Efficiency to have an associated track candidate for signal and background samples, in the central and endcap calorimeters. Because these are rough indications that should be recalculated in the actual sample used, no errors are given.

- $80<M_{\text {inv }}(e, e)<100 \mathrm{GeV}$.

The background sample is selected from EM+jet events, which are dominated by QCD di-jet and $\gamma+$ jet events by requiring:

- Exactly 1 EM cluster, with:

$$
\begin{aligned}
& -p_{T}>20 \mathrm{GeV} \\
& -\chi_{\text {Cal }}^{2}<20, f_{\mathrm{EM}}>0.9, f_{\text {iso }}<0.15
\end{aligned}
$$

- $\not_{T}<15 \mathrm{GeV}, \not_{T}$ is defined in Section 7.3;

- Exactly 1 jet, with $p_{T}>15 \mathrm{GeV}$;

- $\Delta R(e, j e t)>2.5$.

In both samples, we require that the EM object be associated with a track candidate. This association is made to the track with the largest $\operatorname{Prob}\left(\chi_{E M-t r a c k}^{2}, N_{\text {dof }}\right)$ which satisfies an initial selection ${ }^{3}$ :

$$
\left|\Delta \phi_{E M, \text { track }}\right|<0.05,\left|\Delta \eta_{E M, \text { track }}\right|<0.05
$$

The efficiency to be associated with a track candidate is given in Figure 7.2 for both the central region and the endcap calorimeters, and summarized in Table 7.1. After this requirement each of the samples (signal/background, CC/EC) has between 500 and 3000 EM clusters.

\section{Central Calorimeter}

In the central calorimeter, we restrict the EM clusters to $\left|\eta_{D}\right|<1.1$. Distributions of the six variables are given in Figure 7.3 for both signal and background samples. These distributions are used to construct the individual probabilities $P\left(x_{i}\right)$ with which the joint probability $P(\bar{x})$ and the discriminant $D(\bar{x})$ are built. Each of the variables shows 

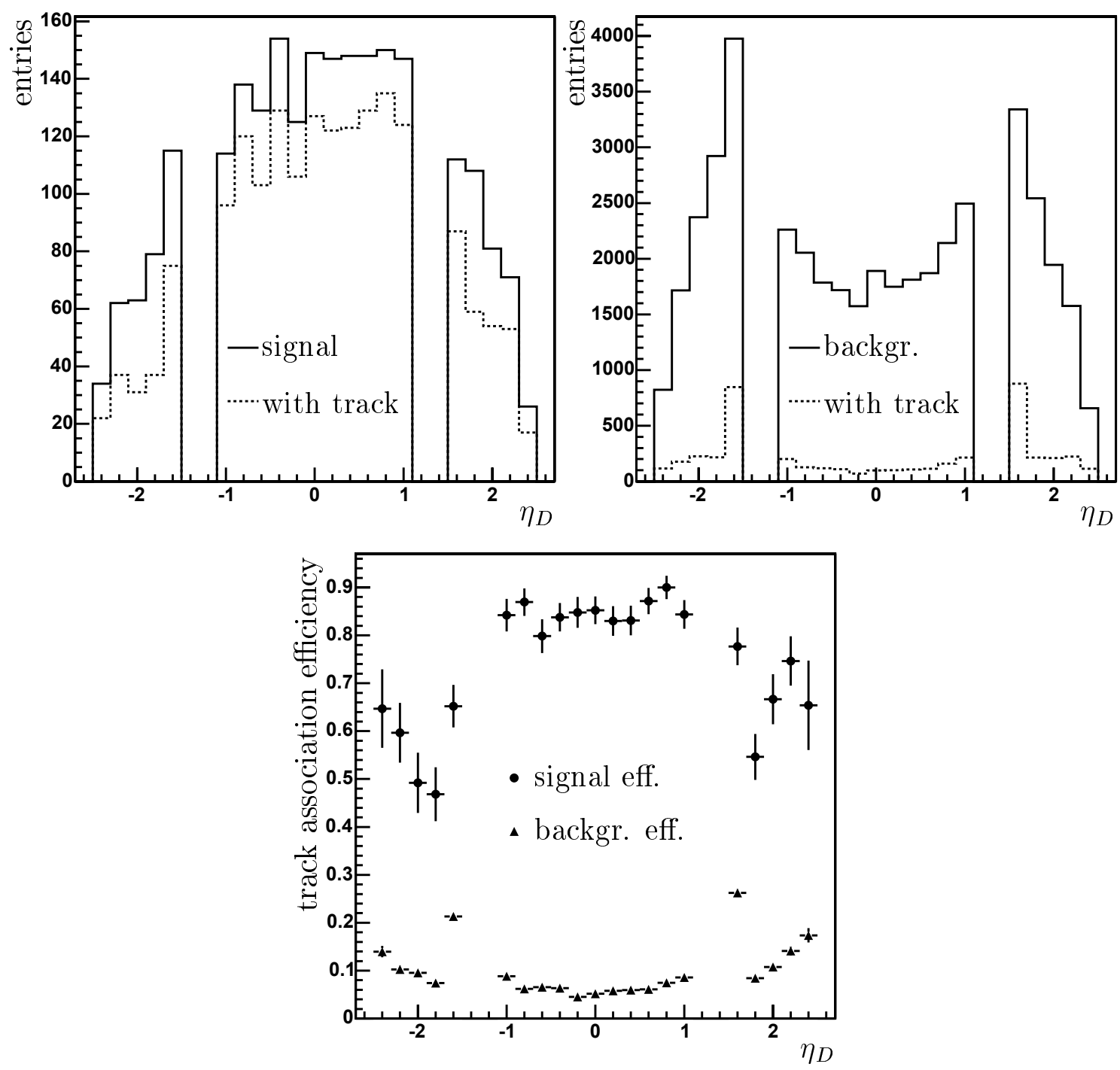

Figure 7.2: The top left and top right plots show all electron candidates and those for which an associated track is found, for signal and background samples respectively, as a function of detector $\eta$. The bottom plot shows the corresponding track association efficiencies. 

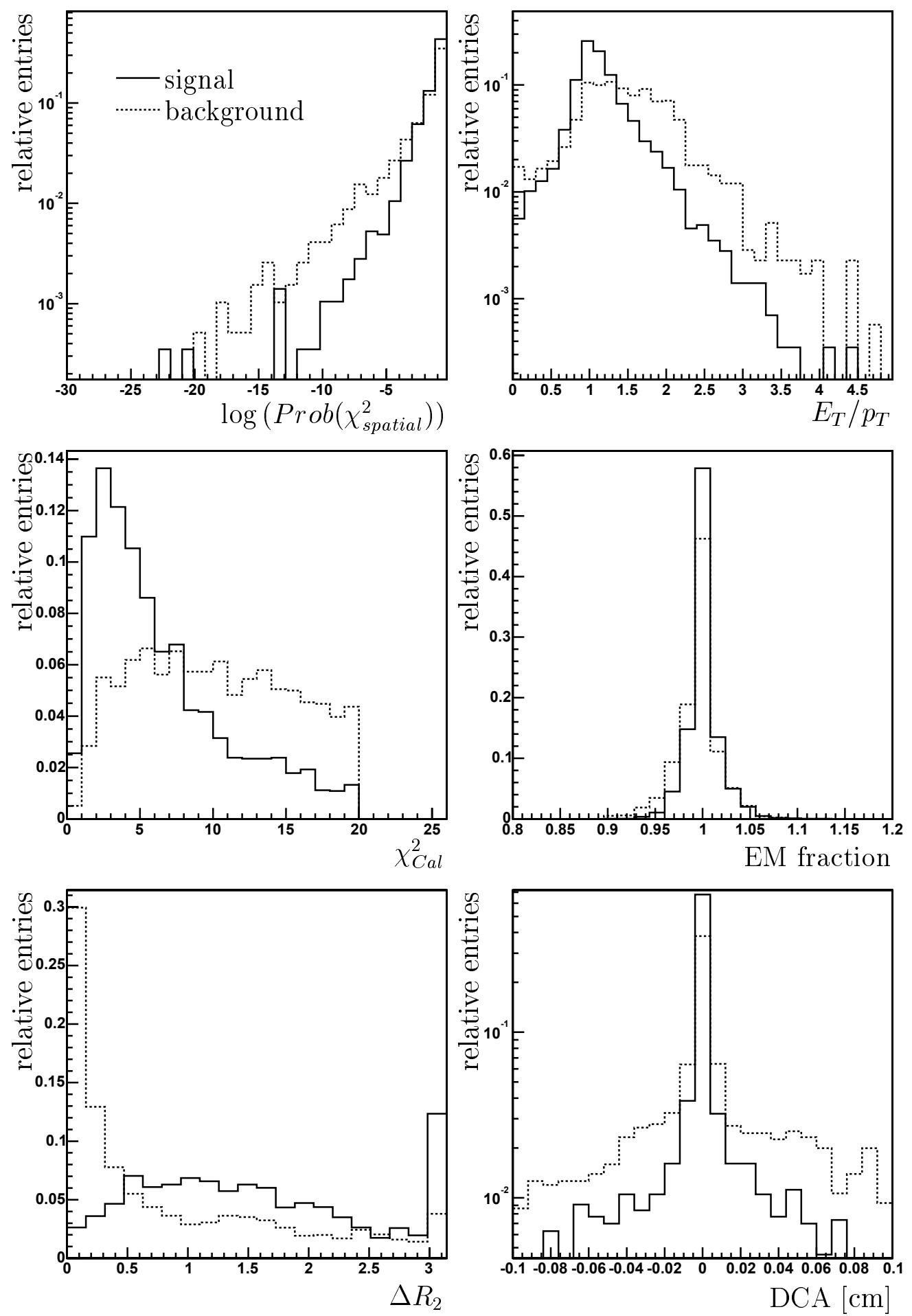

Figure 7.3: Distributions of variables used in the likelihood for central calorimeter signal and backgrounds samples, as defined in the text. Distributions are shown only for EM objects which have a track candidate. 


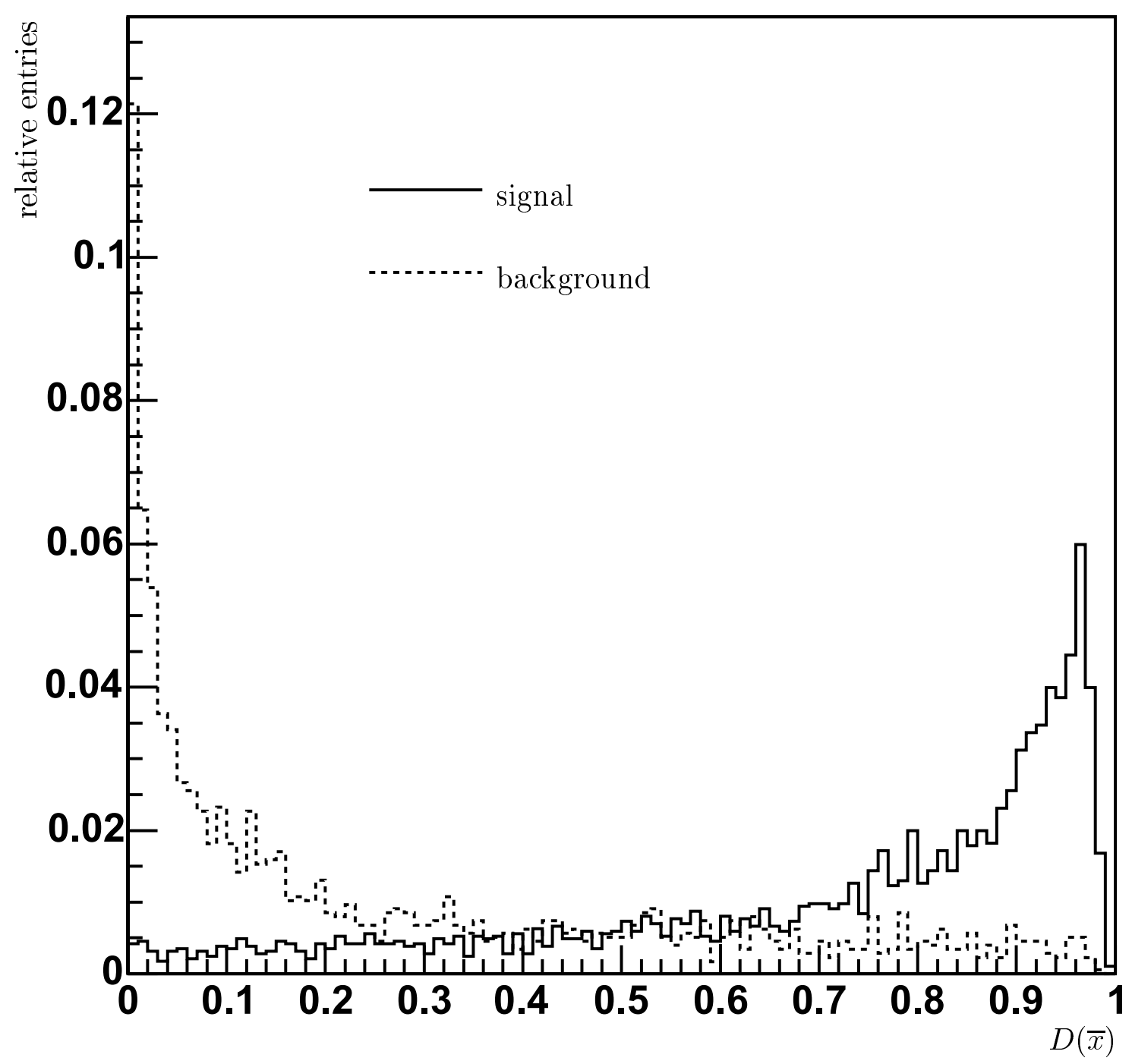

Figure 7.4: Distribution of the discriminant $D(\bar{x})$ for central calorimeter signal and background samples, as defined in the text. The discriminant includes the information of all variables.

some discrimination between the two samples. The distribution of the discriminant $D(\bar{x})$ built of all variables is shown in Figure 7.4 for both signal and background samples.

By varying the cut on the discriminant, both the signal and background efficiency change, giving curves in the signal/background efficiency plane. These curves are

\footnotetext{
${ }^{3}$ The typical resolutions for $\Delta \phi_{E M, t r a c k}$ and $\Delta \eta_{E M, \text { track }}$ for real electrons are 0.008 and 0.007 respectively.
} 


\begin{tabular}{l|r|r}
\hline \hline Threshold & Signal Eff. & Background Eff. \\
\hline \hline \multicolumn{3}{|c}{ Central Calorimeter } \\
\hline \hline$D>0.15$ & $97 \%$ & $50 \%$ \\
\hline$D>0.40$ & $84 \%$ & $25 \%$ \\
\hline \hline \multicolumn{3}{|c}{ Endcap Calorimeter } \\
\hline \hline$D>0.06$ & $96 \%$ & $20 \%$ \\
\hline$D>0.40$ & $88 \%$ & $10 \%$ \\
\hline \hline
\end{tabular}

Table 7.2: Sample selection points and their efficiencies, assuming an associated track. Because these are rough indications that should be recalculated in the actual sample used, no errors are given.

shown in Figure 7.5 for discriminants using different combinations of variables. A simple likelihood which contains only the information regarding the spatial matching and the $E_{T} / p_{T}$ performs just as well as the track matching based on the $\chi_{E M-t r a c k}^{2}$ quantity, with a small improvement in performance at very high efficiencies. Using the full information available in the six variables, we achieve a marked improvement in background rejection at a given electron efficiency. Note that the signal and background efficiencies assume an associated track, the efficiency for which is given in Table 7.1.

We compare the performance of the likelihood to that of cutting on the variables directly by performing a random grid search over cuts on the six likelihood variables. Figure 7.6 shows that the likelihood provides superior performance.

We investigate the discriminating power of each individual variable by comparing the discrimination achieved by the full likelihood to that achieved by the likelihood constructed without that variable. Figure 7.7 shows that each variable, with the exception of $\chi_{\text {spatial }}^{2}$, contributes unique discrimination. That $\chi_{\text {spatial }}^{2}$ doesn't contribute is surprising, because Figure 7.3 shows some discrimination for this variable. This can probably be explained by the fact that other variables (DCA and $E_{T} / p_{T}$ ) contain information about the quality of the track match.

Examples of selection points for the likelihood discriminant and resulting signal and background efficiencies are given in Table 7.2.

\section{Endcap Calorimeter}

In the case of the endcap calorimeters, we restrict ourselves to the region $1.5<\left|\eta_{D}\right|<$ 2.5. Figures 7.8 and 7.9 show the variables and the signal and background efficiencies. Note that the signal and background efficiencies assume a candidate track, the efficiency for which is detailed in Table 7.1. See Table 7.2 for examples of selection points. 


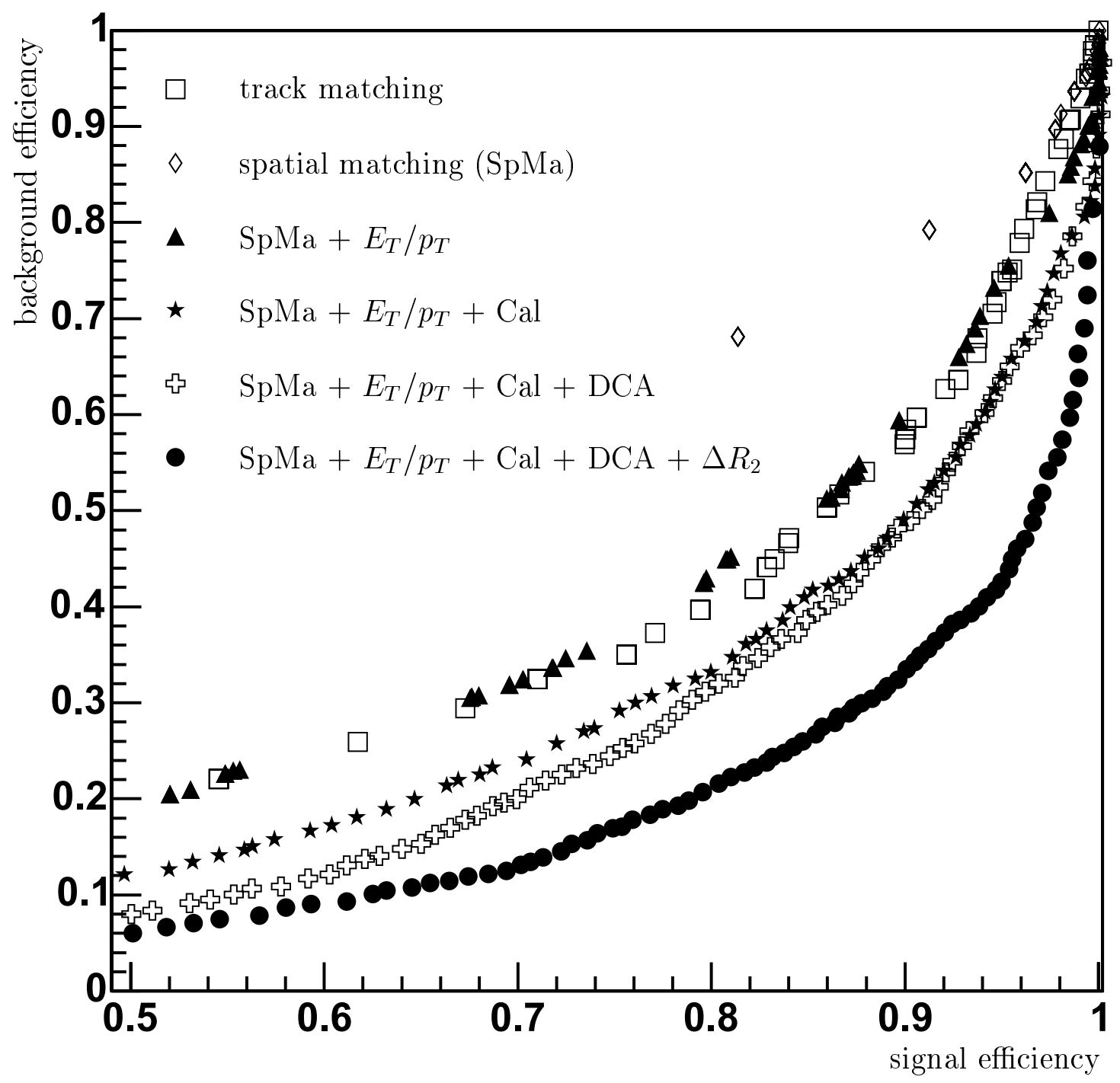

Figure 7.5: Signal and background efficiencies in the central calorimeter for likelihoods using different combinations of variables (Cal means that the calorimeter variables $f_{\mathrm{EM}}$ and $\chi_{\text {Cal }}^{2}$ are included in the likelihood). The curves are produced by varying the thresholds of the discriminants. Note that the signal and background efficiencies assume a candidate track, the efficiency for which is detailed in Table 7.1. 


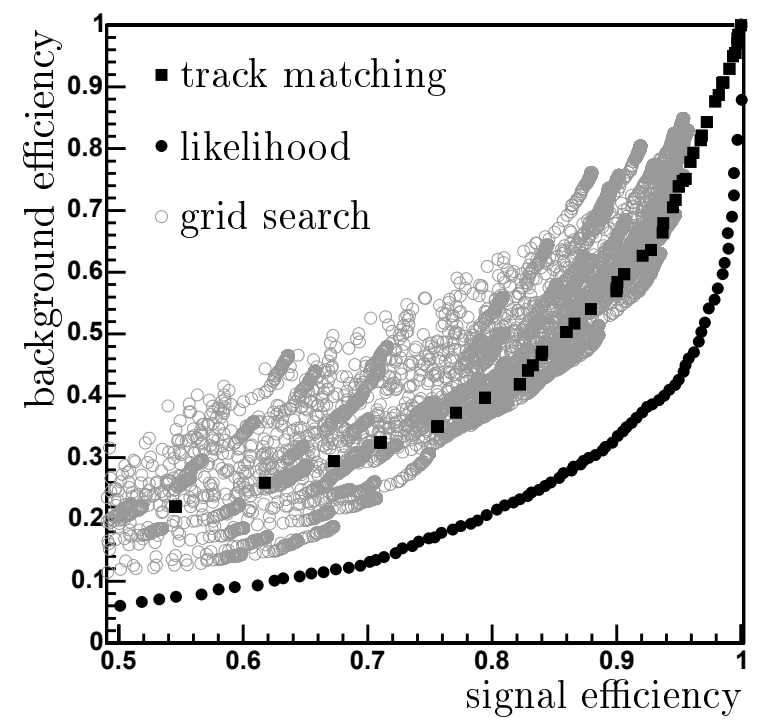

Figure 7.6: A comparison of the performance of the track matching, the likelihood and a grid search in the central calorimeter over the six likelihood variables.

\subsubsection{Likelihood topological dependence}

The construction of the likelihood and its efficiency measurements have been conducted on electrons from the decay of the $Z$. This likelihood discriminant has potential application to other analyses, in which the topology of the event may be very different. We examine the topological dependence of the signal efficiency at a fixed discriminant threshold. Figure 7.10 shows that the efficiency is fairly stable as a function of jet multiplicity, track multiplicity and electron $p_{T}$ and $\eta_{D}$.

\subsubsection{Calorimeter reconstruction efficiency}

The efficiency for finding an EM cluster for an electron in the central calorimeter (the reconstruction efficiency) is measured in data. This is done because the Monte Carlo description of energy deposits in the cracks between the $\mathrm{CC}$ calorimeter modules is known to be unreliable.

We measure this reconstruction efficiency by selecting a $Z \rightarrow e e$ sample, where one of the electrons (the tag side) is required to be found as an electron with a track match, and for the other electron (the probe side) we just require that we find a track. We then find the reconstruction efficiency by counting in how many events the track can be matched (in $\phi$ ) to an EM cluster. The background to the $Z$ events is taken into account by comparing like-sign and unlike-sign electron pairs. Further cleanup of the background is achieved by number of hits requirements on the tracks, and a significance cut on the $\frac{q}{p_{T}}$ (and thus the charge) measurement. 

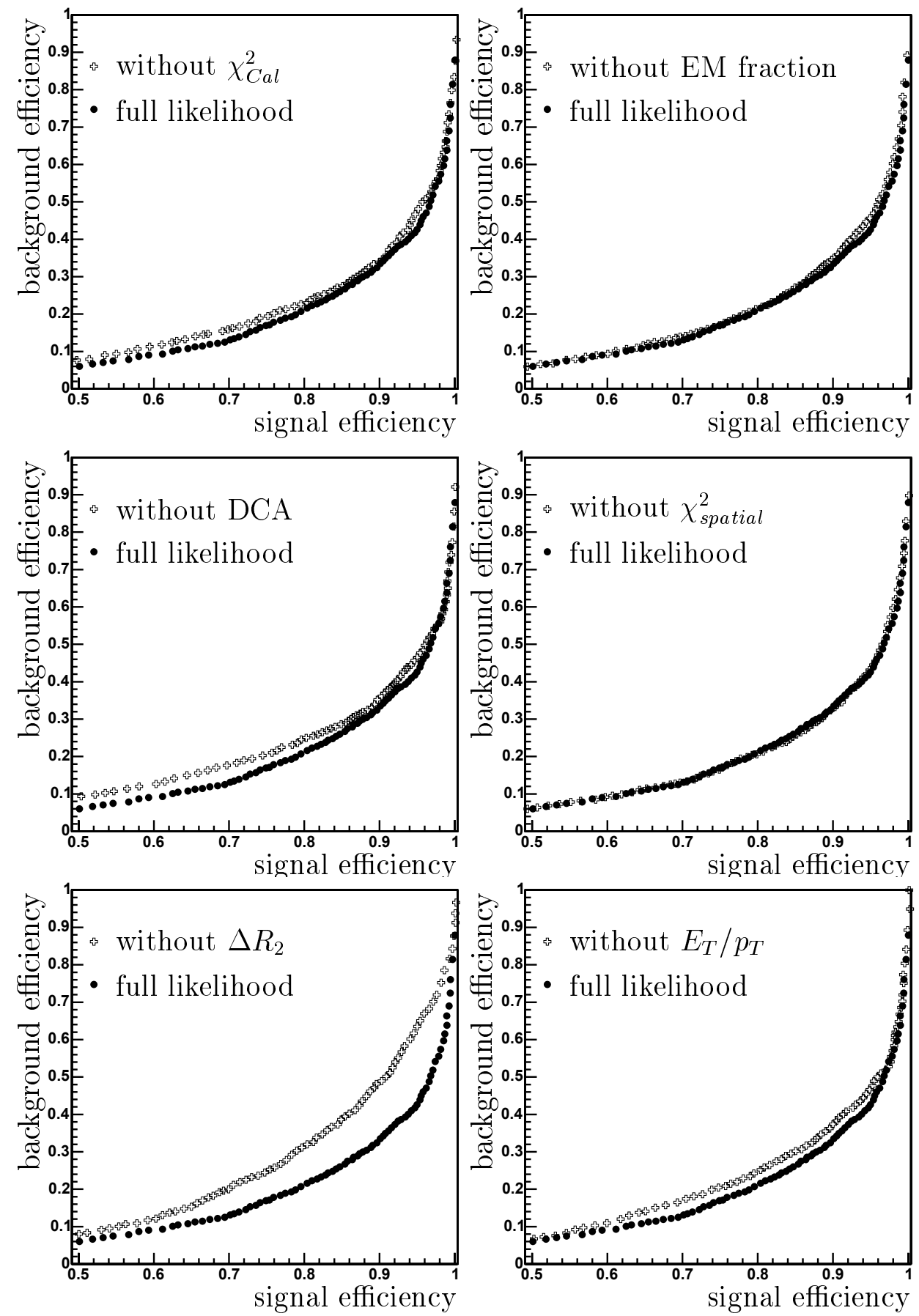

Figure 7.7: Signal and background efficiencies in the central calorimeter for varying thresholds of the discriminants, where information from an individual parameter is removed in order to probe its individual discrimination power. Note that the signal and background efficiencies assume a candidate track, the efficiency for which is detailed in Table 7.1. 

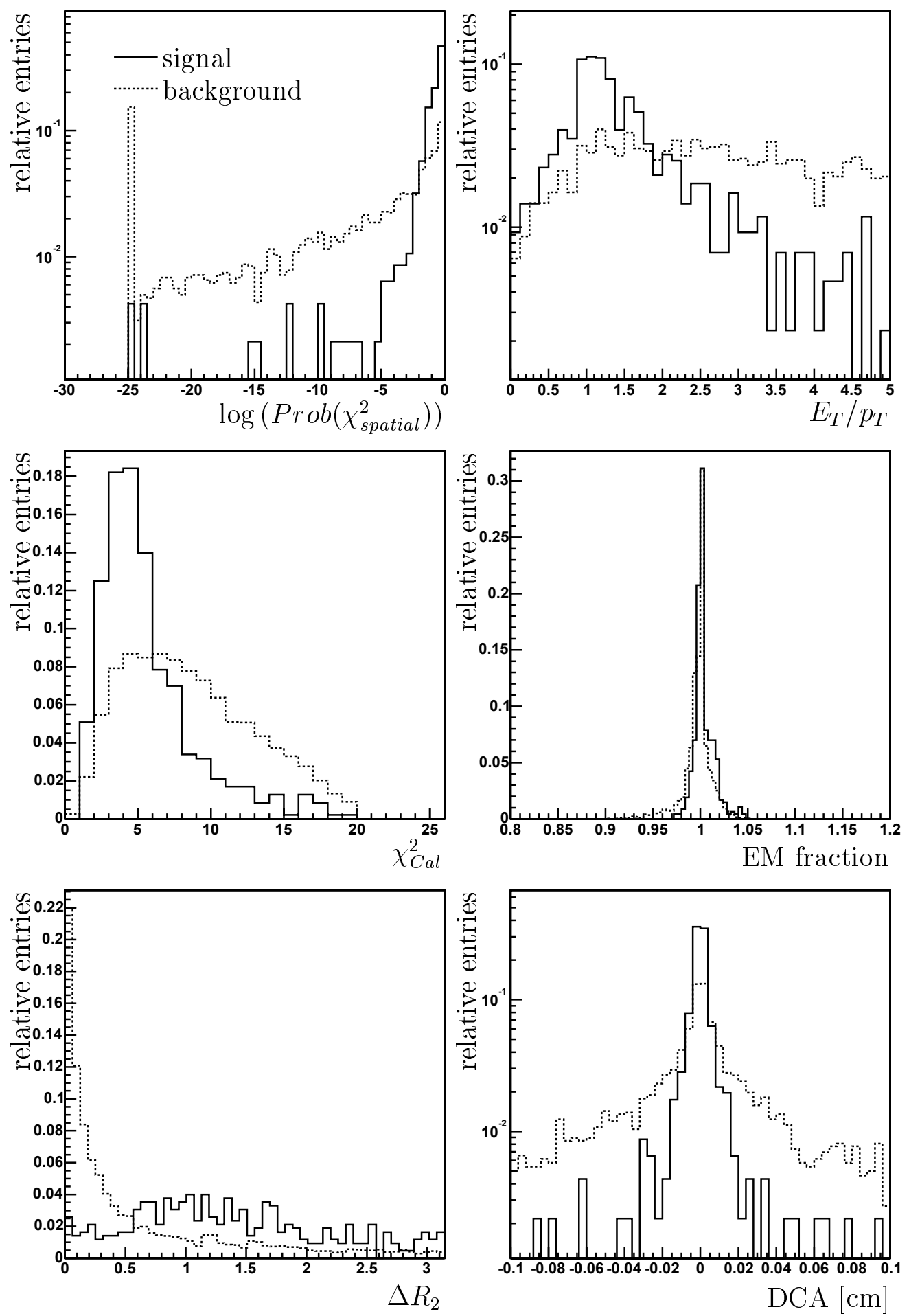

Figure 7.8: Distributions of variables used in the likelihood for endcap calorimeter signal and backgrounds samples, as defined in the text. Distributions are shown only for EM objects which have a track candidate. 


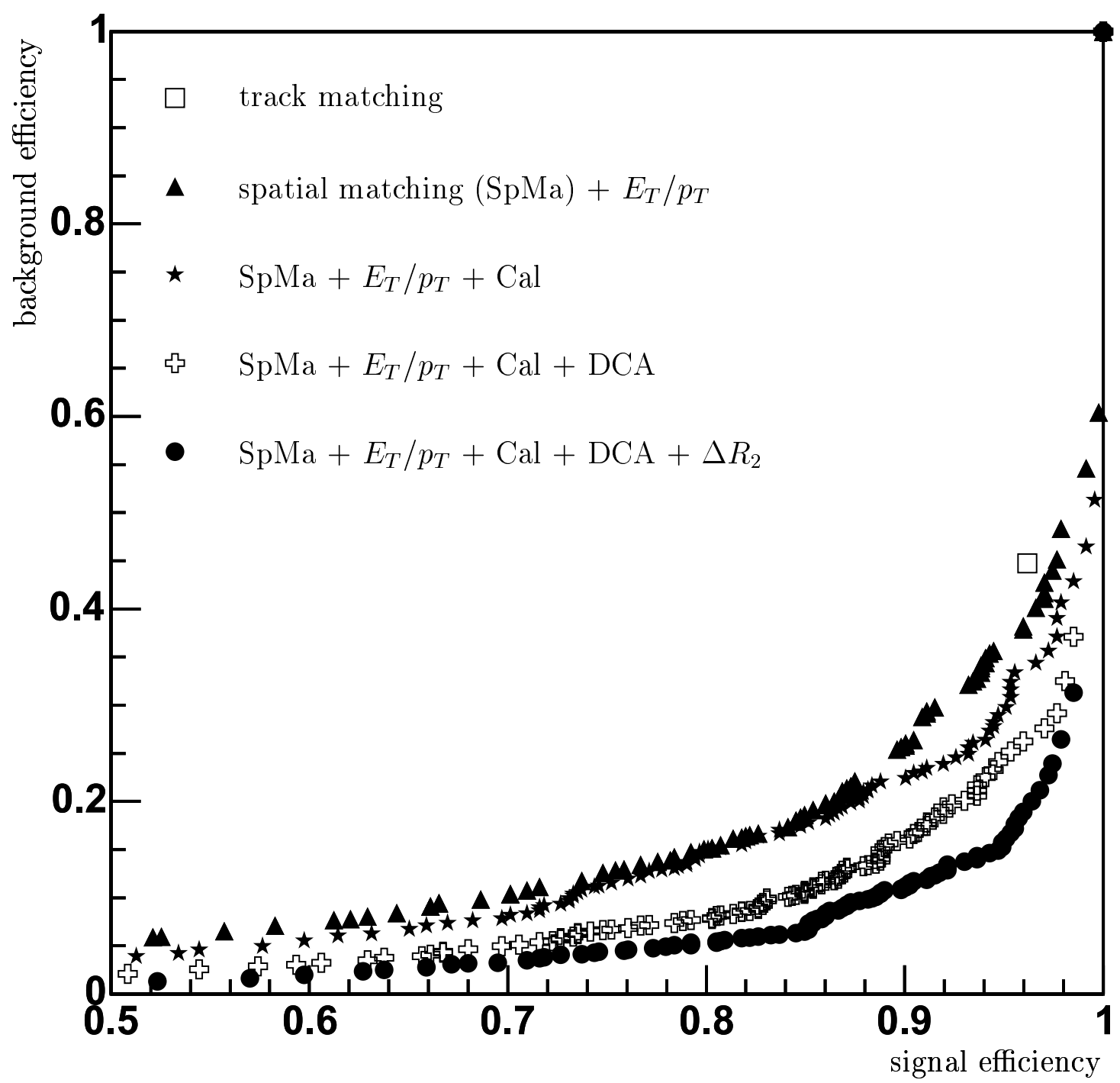

Figure 7.9: Signal and background efficiencies in the endcap calorimeter for likelihoods using different combinations of variables. The curves are produced by varying the thresholds of the discriminants. Note that the signal and background efficiencies assume a candidate track, the efficiency for which is detailed in Table 7.1. 

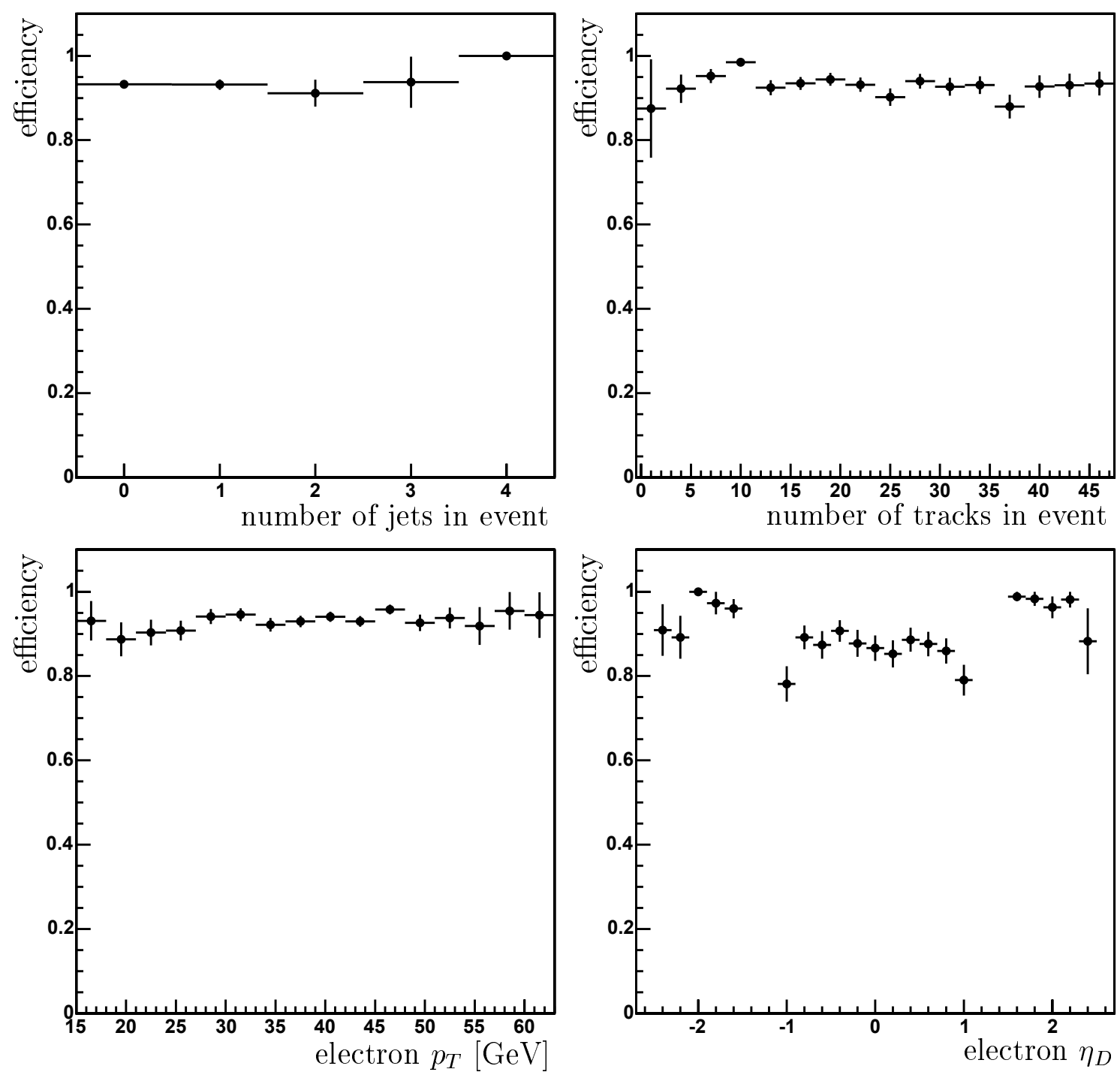

Figure 7.10: The dependence of the signal efficiency in the central calorimeter on a number of topological variables, given a fixed cut on the discriminant. 


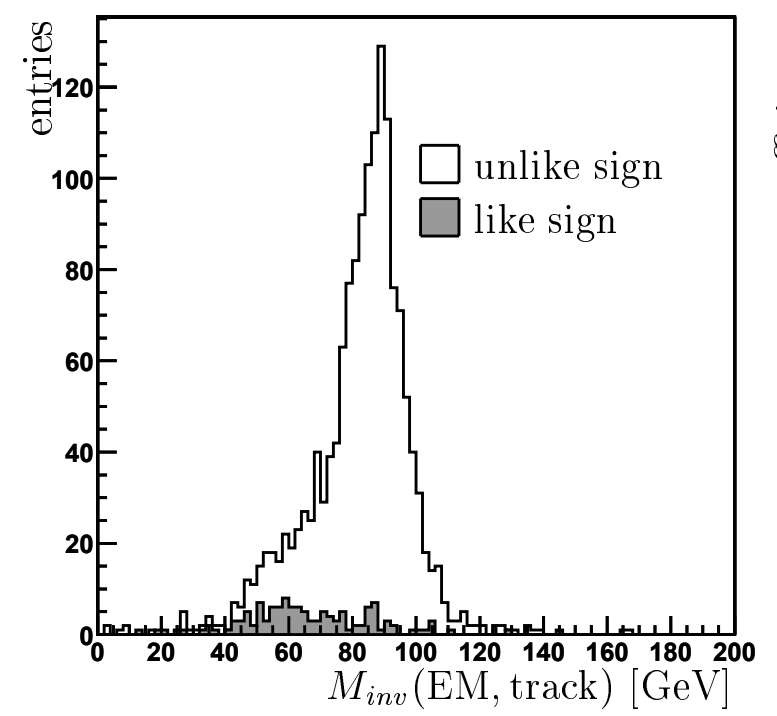

(a)

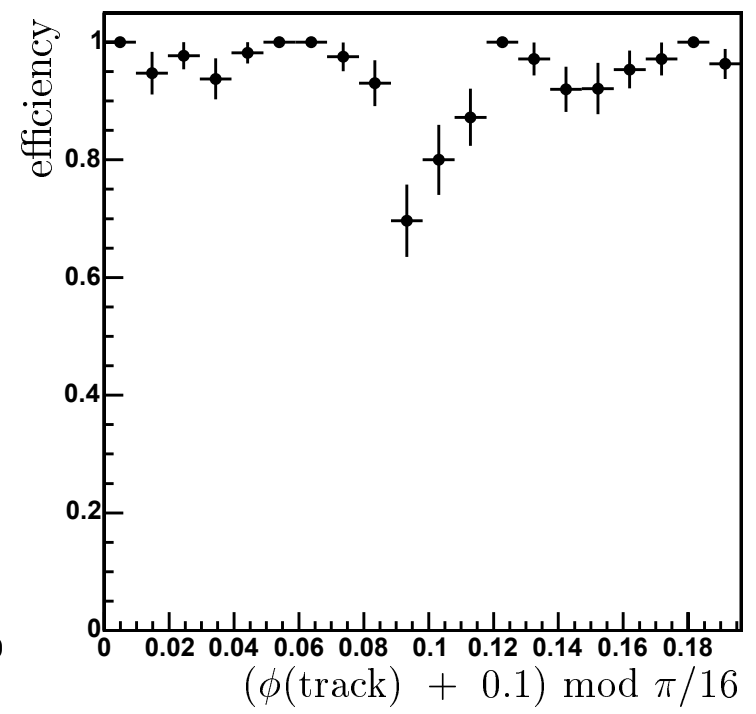

(b)

Figure 7.11: (a) Invariant mass of EM-track pairs, for both like-sign and unlike-sign pairs. (b) The reconstruction efficiency as a function of the modulo of $\phi$ (track), see main text for explanation.

Figure 7.11(a) shows the invariant mass for both the like-sign and unlike-sign EMtrack pairs. It clearly shows the wide $Z$ peak (because of low $p_{T}$ resolution for high $p_{T}$ tracks) and the low amount of background.

Using a narrow mass band for the $Z(80-100 \mathrm{GeV})$, we find a reconstruction efficiency of $95.5 \pm 0.7 \%$ for the CC. For comparison, the MC gives a reconstruction efficiency of $98.8 \pm 0.3 \%$. The measured reconstruction efficiency does not depend on the $z$ position of the electron in the calorimeter (and thus not on $\eta$ ). In the CC, there are small cracks in the $\phi$ direction between the calorimeter modules. The effect of these can be seen in Figure 7.11(b), where we plotted the efficiency as a function of $(\phi($ track $)+0.1) \bmod \pi / 16$, which basically means that all $32 \phi$ modules are overlaid, and the crack should show up at $\phi=0.1$. No background subtraction was used for this plot.

Because there is a slight dependence of the measured reconstruction efficiency on the mass band used, we assign a $2 \%$ systematic uncertainty to the measured number. 


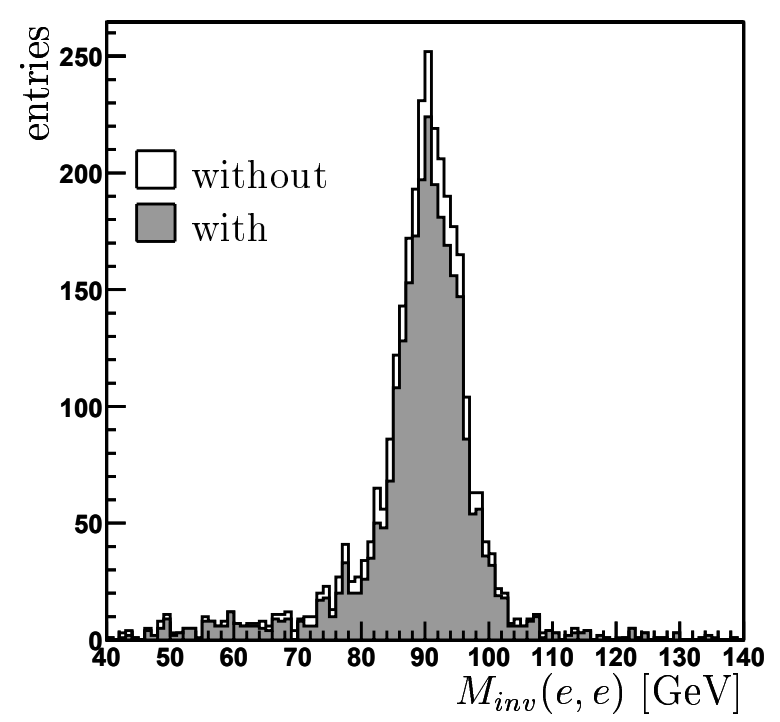

Figure 7.12: Invariant mass of EM pairs, without and with calorimeter cluster selection cuts on the probe electron. For both distributions the tag electron has passed calorimeter selection and likelihood cuts.

\subsubsection{Electron selection efficiency}

The electron selection efficiencies are computed using electrons in the $Z \rightarrow e e$ sample. One of the electrons is used for tagging purposes, and the other (probe electron) is used to evaluate the efficiency. The tag electron is required to pass calorimeter cluster selection and likelihood cuts, so that the sample has little background. The cut under study is then applied to the probe electron. The number of probe electrons that survive this cut gives (after background subtraction) the efficiency for this cut for real electrons. As the systematic uncertainty on this number we take the difference between three methods of estimating the background in the $Z \rightarrow e e$ sample, one using sidebands and the others using linear and exponential fits. We use this method to measure both the cluster selection and the likelihood efficiency.

To give an indication of the statistics available in the $Z$ sample, in Figure 7.12 we show the EM pair invariant mass for all events (which pass the requirements on the tag electron) and those events where the probe passes the calorimeter cluster selection.

\section{Calorimeter cluster selection}

The fraction of electrons satisfying the calorimeter cluster selection cuts $\left(\chi_{\text {Cal }}^{2}<\right.$ $\left.20, f_{\mathrm{EM}}>0.9, f_{\text {iso }}<0.15\right)$ is

$$
\varepsilon_{\mathrm{ID}}=(87.7 \pm 0.7 \text { stat } \pm 1.1 \text { syst }) \%
$$




\begin{tabular}{c|c|c}
\hline \hline & CC & EC \\
\hline \hline$>=0$ jets & $82.1 \pm 0.8$ stat \pm 1.1 syst & $82.9 \pm 1.2$ stat \pm 0.6 syst \\
\hline$>=1$ jets & $79.2 \pm 1.8$ stat \pm 1.0 syst & $81.7 \pm 3.2$ stat \pm 2.0 syst \\
\hline$>=2$ jets & $73.8 \pm 4.3$ stat \pm 6.0 syst & $91.9 \pm 4.5$ stat \pm 8.7 syst \\
\hline \hline
\end{tabular}

Table 7.3: The efficiency (in \%) for electrons that have an associated track to pass the discriminant cut.

in the $\mathrm{CC}$ and

$$
\varepsilon_{\mathrm{ID}}=(97.2 \pm 0.5 \text { stat } \pm 1.3 \mathrm{syst}) \%
$$

in the EC.

\section{Likelihood}

The likelihood requirement consists of two parts, the first is the requirement that the EM candidate is associated with a track, and the second part is the likelihood discriminant ${ }^{4}$ cut itself. The efficiency for an electron to pass both requirements is

$$
\varepsilon_{\mathrm{LH}}=(72.2 \pm 0.8 \text { stat } \pm 0.9 \text { syst }) \%
$$

in the $\mathrm{CC}$ and

$$
\varepsilon_{\mathrm{LH}}=(53.2 \pm 1.3 \mathrm{stat} \pm 0.2 \mathrm{syst}) \%
$$

in the $\mathrm{EC}^{5}$. We are also interested in the efficiency for just the likelihood discriminant cut, because in the next chapter we will define a sample of loose electrons as those that pass the calorimeter cluster selection criteria and have a track associated with them, and a sample of tight electrons as the ones that also pass the discriminant cut. We need to know the efficiency for electrons to go from loose to tight, which basically is the efficiency for electrons (that have a track associated) to pass the discriminant cut. This efficiency is shown in Table 7.3. There is no significant dependence on the number of jets, which was already shown in Figure 7.10.

\footnotetext{
${ }^{4}$ We have chosen a cut of $D>0.4$. This point was chosen such that the efficiency of the likelihood is the same as that of the central track confirmation, which was used in a previous version of this analysis.

${ }^{5}$ The efficiencies found here are slightly lower than the ones given in Tables 7.1 and 7.2. This is because those efficiencies were derived on the sample that the likelihood was trained on, where the efficiencies given here are derived from the full data sample.
} 


\subsubsection{Electron energy corrections}

The energy lost by electrons in the material in front of the calorimeter (solenoid and preshower) has been studied using a detailed simulation. It is parameterized as a function of $\eta$ and electron energy. This parameterization is used to correct the data.

Energy corrections for the energy lost in the $\phi$ cracks between the CC modules are also applied.

\subsection{Jets}

\subsubsection{Jet reconstruction}

Jets are reconstructed at D $\varnothing$ using the improved legacy cone algorithm [112] designed following the recommendation of the Run2 QCD workshop.

In the first step of this algorithm, energy deposits are segmented in towers of $0.1 \times 0.1$ in $\eta \times \phi$. If the transverse energy of a tower is above $0.5 \mathrm{GeV}$, it is used as a seed for the precluster stage, in which preclusters are formed with a cone size of $R_{\text {precluster }}=$ $\sqrt{\Delta \eta^{2}+\Delta \phi^{2}}=0.3$.

In the next step, all preclusters with $E_{T}>1 \mathrm{GeV}$ in a cone of size $R$ are combined to give the starting points of the final jets. The direction of these starting points is given by the $E_{T}$ weighted average of the $\eta$ and $\phi$ of the preclusters.

Next, the energy deposited within a cone of size $R$ around these starting points is accumulated, and the new $(\eta, \phi)$ direction is calculated by $E_{T}$ weighted average of the energy deposits. This step is repeated until the direction of the jets is stable.

If two of these stable jets are separated by more than $R$ but less than $2 R$, a new jet axis is defined at the midpoint of the two jets, which is then used as a precluster to try and reconstruct a jet around.

Finally, if two jets share more energy than half the energy of the lowest energy jet, they are merged. If less energy is shared, each of the shared clusters is assigned to the closest jet.

For the cross section measurement in this thesis, a cone size of $R=0.5$ is chosen.

Once jets are clustered with the cone algorithm, further quality selection cuts are applied to each jet. These criteria are aimed at removing fake jets:

- To remove isolated electromagnetic particles a cut on the fraction of energy deposited in the electromagnetic section of the calorimeter $\left(f_{\mathrm{EM}}\right)$ is applied at $0.05<f_{\mathrm{EM}}<0.95$.

- To remove jets which predominantly deposit their energy in the coarse hadronic section of the calorimeter, a cut on the fraction of the jet energy deposited therein 
$(\mathrm{CHF})$ is applied at $C H F<0.4$. This cut is essentially aimed at removing those jets which cluster around noise in the coarse hadronic section.

- To remove jets clustered from hot cells, a cut on the ratio of the highest to the next-to-highest transverse energy cell in the jet $(H o t F)$ is applied at $H o t F<10$.

- To remove jets clustered from a single hot tower, the number of towers containing $90 \%$ of the jet energy $(n 90)$ is required to be greater than one.

\subsubsection{Jet Energy Scale and resolution}

Because of non-linearities, dead material, noise and showering effects, the measured energy in a jet cone is not equal to the original particle level energy. Since the Monte Carlo simulation may not model these effects accurately, the scale of jets in data and in Monte Carlo can be different. The jet energy scale corrections attempt to correct the measured energy of jets back to the energy of the particles in the jets before their interaction with the detector ${ }^{6}$.

Jet energies are corrected using:

$$
E_{\text {corrected }}=\frac{E_{\text {measured }}-O}{\mathcal{R} \times S} .
$$

Here, $\mathcal{R}$ is the calorimeter response to a jet; it is determined using transverse energy balance in photon+jet events. $O$ is the energy offset due to the underlying event, energy pile-up, multiple interactions, electronic noise, and uranium noise from the uranium absorber. $O$ is determined by energy densities in minimum bias events. $S$ is the fraction of shower leakage outside the jet cone of $R=0.5$ in the calorimeter. This is determined by measuring the energy profile of jets in photon+jet events. In the analysis in this thesis we use the JetCorr v4.2 [114] package which incorporates all three components of the jet energy scale. It provides corrections for data as well as MC. Statistical and systematic errors are also provided so that we may investigate their effect on our overall signal and background efficiencies.

The energy resolution of jets in data and Monte Carlo is significantly different. This can be seen in [115], which gives the measured data and Monte Carlo jet resolutions for different $\eta$ ranges. To correct for this, we smear the Monte Carlo jets such that the resolution after smearing is the same as the resolution measured in data. As an example, the additional energy smearing needed for a Monte Carlo jet with a $p_{T}$ of 50 $\mathrm{GeV}$ at $\eta=0$ is about $8 \%$.

\footnotetext{
${ }^{6}$ A detailed description of the method applied to data from the 1992-1995 run of the DØ experiment can be found in [113].
} 


\subsubsection{Rejection of noise jets}

Despite the numerous quality requirements applied to avoid clustering jets on noise in the calorimeter, a large number of jets are selected seemingly due to noise. These noise jets originate from low energy but high occupancy noise in well confined regions of the calorimeter.

As these jets have their energy more or less evenly spread throughout all their towers, $n 90$ could be used to discriminate them against good jets. But in the case of very wide jets, for example with soft gluon radiation in the final state, $n 90$ could be very large as well. To avoid rejecting these jets, a more suitable discriminating variable would thus be $f 90$ which is the ratio of $n 90$ and the number of towers that form the jet.

It was found, however, that $n 90$ (and thus $f 90$ ) are calculated incorrectly for merged jets in the version of the reconstruction code used in this analysis ${ }^{7}$. But, doing a L1 calorimeter comparison ${ }^{8}$ helped to demonstrate that many of the high $f 90$ jets in actual data are fake. Further, it was determined that the number of fakes increases as one goes to lower $p_{T}$ or higher coarse hadronic fraction within the high $f 90$ population of jets. Conversely, low $C H F$ jets regardless of $f 90$ value appear to be good jets. This study is described in more detail in [116].

A two-dimensional selection in two $p_{T}$ bins was determined to effectively reject the observed fake background. We reject all jets that do not satisfy:

- if jet $p_{T}<25 \mathrm{GeV}: f 90<0.7-0.5 \times C H F$ or $C H F<0.025$;

- if jet $p_{T}>25 \mathrm{GeV}: f 90<0.8-0.5 \times C H F$ or $C H F<0.05$.

\subsubsection{Efficiency of noise rejection cuts}

Because of our aggressive attempts to suppress noise problems in the calorimeter with the cuts of Section 7.2.3, we must estimate what efficiency these cuts have for real jets. As already pointed out, the $f 90$ variable, which is used to qualify jets, is not calculated correctly for merged jets in the version of the reconstruction code used in this analysis.

\footnotetext{
${ }^{7}$ Although it is possible to recalculate $n 90$ for each jet, this would be cumbersome because it requires redoing the full analysis. It was thus decided to use the incorrect $n 90$ variable, making sure it is used consistently.

${ }^{8}$ The energy in the jet is compared with the energy that the trigger readout finds in the jet cone. The trigger readout is separate from the precision readout and does not suffer from the same problems which have been observed to produce noise jets. It can therefore be used to identify if the jet is real or fake. Since the gain of the trigger readout is low in the ICD region, the efficiency for identifying jets using this method is low in this region of the detector. But, in other detector regions it can be used to define pure samples of real and fake jets, which in turn can be used to study fake jet rejection cuts.
} 


\begin{tabular}{c|c|c|c}
\hline \hline & all & CC & EC \\
\hline \hline data & $96.6 \%$ & $95.7 \%$ & $97.7 \%$ \\
\hline $\mathrm{MC}$ & $97.2 \%$ & $96.5 \%$ & $97.8 \%$ \\
\hline \hline
\end{tabular}

Table 7.4: Noise rejection cut efficiency for jets in photon + jet sample in data and MC.

But as both the sample on which this efficiency is measured, and the final data sample have this incorrect $f 90$ variable, the final efficiency will be calculated correctly.

We study the efficiency of the noise rejection cuts in a photon + jet sample. The selection criteria are:

- a photon candidate $\left(p_{T}>15 \mathrm{GeV}, \chi_{C a l}^{2}<20, f_{E M}>0.9, f_{\text {iso }}<0.15\right)$;

- a jet with $\Delta \phi(\gamma$, jet $)>3.0,|\eta|<2.5, p_{T}>15 \mathrm{GeV}$ and (in data) a matched L2 jet $\left(E_{T}>3 \mathrm{GeV}\right)$;

- $\not_{T}<10 \mathrm{GeV}, \not_{T}$ is defined in Section 7.3.

These criteria, including the $\Delta \phi$ and L2 requirements, are chosen to minimize the presence of fake jets in this sample. We then measure the efficiency by applying the noise rejection cuts to the jet and measuring what fraction of the jets survives. The results are given in Table 7.4, and are shown as a function of $p_{T}$ and $\eta$ in Figure 7.13. A similar behavior is observed in both data and Monte Carlo.

\subsubsection{Jet/EM separation}

EM clusters with a $p_{T}$ of $8 \mathrm{GeV}$ or higher are also reconstructed as jets. If the EM cluster is a real electron or photon, we do not want to use this jet, because it is just a reclustering of the energy in the calorimeter that belongs to the electron. Thus we drop all jets which are within $\Delta R$ of 0.5 of an electron candidate ${ }^{9}$ with $p_{T}>15 \mathrm{GeV}$.

\subsection{Missing transverse energy $E_{T}$}

Even with a perfect detector, there will not be energy balance in the plane transverse to the beam if there are neutrinos produced in the event. This is because the neutrinos are not observed by the detector. Thus, the presence of a neutrino in the final state can be detected from the energy imbalance of an event in the transverse plane. In a real detector, also the finite detector resolution contributes to this imbalance.

\footnotetext{
${ }^{9}$ An EM cluster that satisfies $\chi_{\text {Cal }}^{2}<20, f_{\mathrm{EM}}>0.9, f_{\text {iso }}<0.15$.
} 


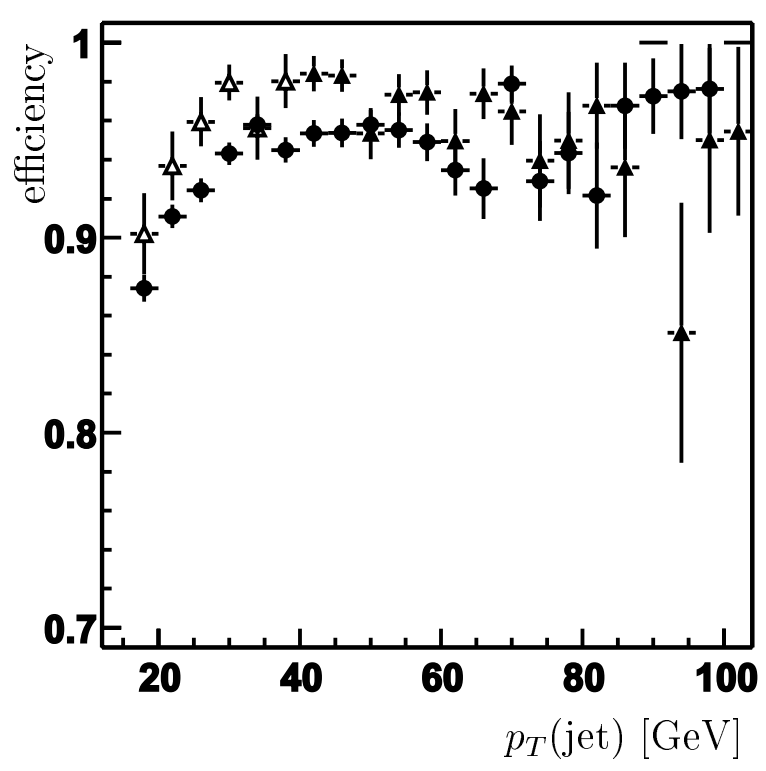

(a)

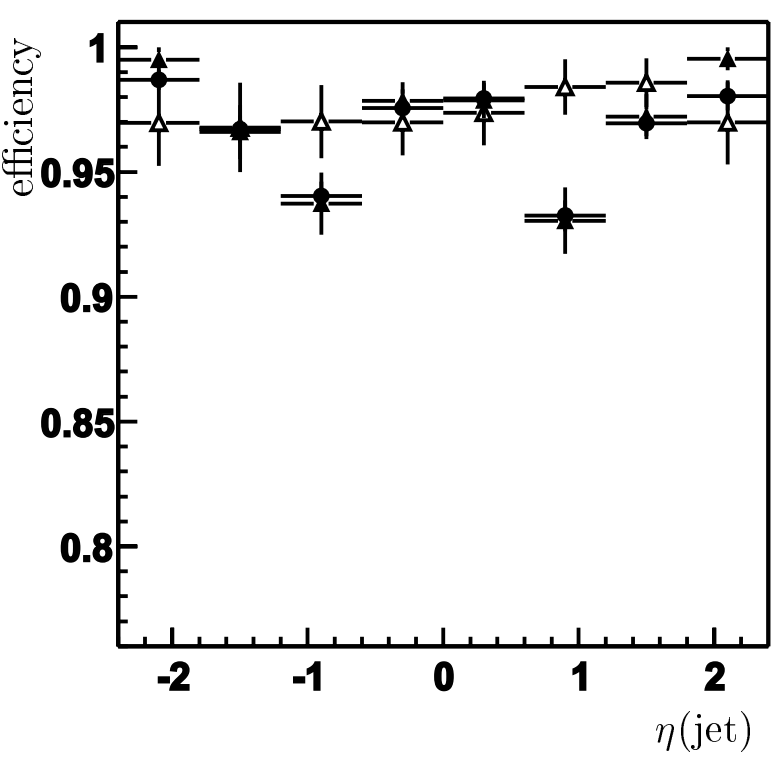

(b)

Figure 7.13: Efficiency for real jets to survive the noise rejection cuts in data (filled circles) and $M C$ (filled and unfilled triangles) as a function of jet $p_{T}$ (a) and $\eta(b)$. The unfilled triangles are a $M C$ sample of jets with $p_{T}<40 \mathrm{GeV}$, while the filled triangles are a sample of jets with $p_{T}>40 \mathrm{GeV}$.

This energy imbalance is calculated by taking the vector sum of the transverse energies of all cells (with transverse energy in excess of $100 \mathrm{MeV}$ ) in all layers of the calorimeter except for those in the coarse hadronic layer which are treated separately due to their high level of noise. The only cells of the coarse hadronic layer which are accounted for in the aforementioned sum are those clustered within good jets. The vector opposite to this vector is the raw missing transverse energy vector.

The calorimeter response to electromagnetic particles, such as photons and electrons, is different from that to jets. In events with both electromagnetic objects and jets, this imbalance translates into missing transverse energy. The response of the calorimeter to jets (JES), which has already been used to correct the jets, can also be used to correct the missing transverse energy. In order to do so, the JES correction (limited to the response part of such correction) for all good jets is subtracted from the $E_{T}$ vector.

A similar correction to the $\mathbb{E}_{T}$ is made for the electron energy corrections, which were discussed in Section 7.1.8. This is done by subtracting the corrected transverse momentum of the electrons from the $\not_{T}$ vector, where the $\not_{T}$ has now been calculated without taking the calorimeter cells in account that were used to form the electron 
clusters. The resulting quantity is the calorimeter missing transverse energy.

As a muon is a minimum ionizing particle, it will deposit only a small amount of energy in the calorimeter. Its presence can thus also fake missing transverse energy in the calorimeter. The momentum of all muons present in the event is subtracted from the missing transverse energy vector after deduction of the expected energy deposition by the muon in the calorimeter (which are derived from Monte Carlo simulation). This gives the final missing transverse energy $\mathbb{E}_{T}$. 


\section{Chapter 8}

\section{Top pair cross section analysis}

\subsection{Introduction}

We will now turn our attention to measuring the $t \bar{t}$ cross section. The data used for this measurement was taken between August 22, 2002 and June 24, 2003. The integrated luminosity of the data sample used is $92 \mathrm{pb}^{-1}$. This is after all runs and luminosity blocks ${ }^{1}$ with known problems have been removed.

The measurement described in this thesis will be in the channel where one of the $W^{\prime}$ 's (coming from the top) decays to an electron and a neutrino, and the other decays to hadrons (the so-called electron+jets channel). This means that we are looking for the following event signature:

- isolated electron (from the $W$ decay);

- missing transverse momentum $\not_{T}$ (evidence for the unseen neutrino);

- four or more jets.

The two main backgrounds are $W+$ jets production (where the $W$ decays to an electron and a neutrino) and QCD multi-jet events with a misidentified electron and mismeasured $E_{T}$. Other possible backgrounds are $W+$ jets events where the $W$ decays first to a tau which subsequently decays to an electron, and $Z+$ jets events. The $Z$ can decay to electrons and if one of the electrons is either missed or not identified as an electron candidate, this can have the correct event signature. If the $Z$ decays to taus, and one of the taus decays hadronically and the other to an electron and two neutrinos, this can also mimic the event signature. In Appendix $\mathrm{C}$ we show that these backgrounds do not need to be treated separately, because either they are so small that

\footnotetext{
${ }^{1}$ A luminosity block is the smallest part of a run (about one minute long) for which the luminosity can be calculated.
} 
they can be ignored, or they are treated correctly by lumping them together with the main $W+$ jets background.

The analysis of this particular decay channel of $t \bar{t}$ is performed in two steps. The first step consists of defining a data sample enriched in $W+$ jets and top events, within which the absolute normalization of backgrounds from both $W+$ jets and QCD multijet events is evaluated. In a second step, further kinematic cuts (based on the topology of the events) are applied to reach the final selection. In order to be able to combine the result of this topological approach with results from an independent analysis using a soft muon b-tagging approach, a soft muon tag veto is applied in the analysis to avoid overlap.

In the current analysis, we look only at events where the electron ends up in the Central Calorimeter (CC). The reason for this will become clear in Section 8.3.

\subsection{The $\mathrm{W}+$ jets selections}

We start with a rather loose preselection of $\mathrm{W}$ and top events. The basic requirements for these samples are an electron candidate $\left(p_{T}>20 \mathrm{GeV}\right.$, with an associated track) and $\not_{T}$. In addition we require one or more jets. This way, we define four loose samples based on the inclusive jet multiplicity (up to four) of the event. The one jet sample has one or more jets, the two jet sample has two or more jets, etc. A list of all the selection cuts used to define these loose samples is given in Table 8.1. A detailed explanation for each of these cuts can be found in Section 8.7. For each of these loose samples, we define a corresponding tight sample by the additional requirement that the electron in the event passes the likelihood cut. These tight samples are thus subsets of the loose samples.

\subsection{QCD background}

We will now estimate the QCD background in the four tight samples. This is done using the properties of the electron candidate in the event. The $W+$ jets and $t \bar{t}$ events have real isolated electrons, while the electron candidate in QCD events are either non-isolated or fake (a $\pi^{0}$ shower with an overlapping track from a charged particle or a photon converting to a $e^{+} e^{-}$pair). For ease of notation, we will use " $W+t \bar{t}$ events" when we mean the sum of the $W+$ jets and $t \bar{t}$ events (and thus $N^{\mathrm{W}+\mathrm{t} \overline{\mathrm{t}}} \equiv N^{\mathrm{W}}+N^{\mathrm{t} \overline{\mathrm{t}}}$ ).

We write the number of events in the loose sample $\left(N_{\text {loose }}\right)$ as the sum of the number of $W+t \bar{t}$ and QCD events:

$$
N_{\text {loose }}=N^{\mathrm{W}+\mathrm{t} \overline{\mathrm{t}}}+N^{\mathrm{QCD}} .
$$




\begin{tabular}{|c|}
\hline \hline Loose selection criteria \\
\hline \hline EM particle, $p_{T}>20 \mathrm{GeV},\left|\eta_{D}\right|<1.1$ \\
\hline electron passes calorimeter cluster selection \\
\hline electron has an associated track \\
\hline soft muon tag veto \\
\hline second electron veto \\
\hline$\not_{T}>20 \mathrm{GeV}$ \\
\hline$\Delta \phi\left(\not_{T}, e\right)>0.5$ \\
\hline Primary vertex with $\geq 3$ tracks and $|z|<60 \mathrm{~cm}$ \\
\hline$N_{\text {jets }} \geq 1,2,3,4\left(\right.$ with $\left.p_{T}>15 \mathrm{GeV},|\eta|<2.5\right)$ \\
\hline
\end{tabular}

\begin{tabular}{|c|}
\hline \hline Tight selection criteria \\
\hline \hline loose criteria + electron passes likelihood \\
\hline \hline
\end{tabular}

Table 8.1: Loose and tight selection criteria.

This is of course also true for the tight sample, but the contributions from $W+t \bar{t}$ and QCD are reduced by the additional requirement of the likelihood cut. But, we expect the amount of reduction to depend on the type of electron candidate, and thus to be different for $W+t \bar{t}$ and QCD. These reductions are actually the efficiencies for electron candidates in the $W+t \bar{t}$ and QCD samples to pass the likelihood requirement. The number of events in the tight sample $\left(N_{\text {tight }}\right)$ can thus be written as:

$$
\begin{aligned}
N_{\text {tight }} & =\varepsilon_{\text {sig }} N^{\mathrm{W}+\mathrm{t} \overline{\mathrm{t}}}+\varepsilon_{Q C D} N^{\mathrm{QCD}} \\
& \equiv N_{\text {tight }}^{\mathrm{W}+\mathrm{t} \overline{\mathrm{t}}}+N_{\text {tight }}^{\mathrm{QCD}} .
\end{aligned}
$$

If these efficiencies $\left(\varepsilon_{\text {sig }}\right.$ and $\left.\varepsilon_{Q C D}\right)$ were known, we could solve these two equations for $N^{\mathrm{W}+\mathrm{t} \overline{\mathrm{t}}}$ and $N^{\mathrm{QCD}}$, and thus get a prediction for the $W+t \bar{t}$ and QCD events in the tight sample:

$$
N_{\text {tight }}^{\mathrm{W}+\mathrm{t} \overline{\mathrm{t}}}=\varepsilon_{\text {sig }} \frac{N_{\text {tight }}-\varepsilon_{Q C D} N_{\text {loose }}}{\varepsilon_{\text {sig }}-\varepsilon_{Q C D}} \quad \text { and } \quad N_{\text {tight }}^{\mathrm{QCD}}=\varepsilon_{Q C D} \frac{\varepsilon_{\text {sig }} N_{\text {loose }}-N_{\text {tight }}}{\varepsilon_{\text {sig }}-\varepsilon_{Q C D}} .
$$

This clearly only works if $\varepsilon_{s i g}$ and $\varepsilon_{Q C D}$ are sufficiently different from each other. We expect $\varepsilon_{s i g}$ to be much higher than $\varepsilon_{Q C D}$, because the likelihood was trained to distinguish real from fake electrons. We will now show that these efficiencies can be derived from data, and that we can therefore use this method to extract the number of QCD background events in each of the four loose and tight samples. 


\begin{tabular}{c|c}
\hline \hline & $\varepsilon_{Q C D}$ \\
\hline \hline$\geq 1$ jet & $0.202 \pm 0.003$ \\
\hline$\geq 2$ jets & $0.210 \pm 0.006$ \\
\hline$\geq 3$ jets & $0.207 \pm 0.013$ \\
\hline$\geq 4$ jets & $0.197 \pm 0.034$ \\
\hline \hline
\end{tabular}

Table 8.2: $\varepsilon_{Q C D}$ for different inclusive jet multiplicities.

$\varepsilon_{Q C D}$

Figure 8.1 shows the ratio of the number of events in the loose and the tight samples (but without the $\mathbb{E}_{T}$ cut), as a function of $\mathbb{E}_{T}$, for each of the four jet multiplicities. We expect the low $\mathbb{E}_{T}$ region to be dominated by QCD, because the contributions from real isolated electrons all have nonzero $\mathbb{E}_{T}$. In addition, we expect these QCD fake electrons to be independent of the $\mathbb{E}_{T}$ of the event, because the $\mathbb{E}_{T}$ in these events originates from mismeasured jet energies, which are independent of the mechanism that produces these fake electrons. At higher $\not_{T}$, there is a contribution from the real electrons coming from $W$ 's (and at higher jet multiplicities also from $t \bar{t}$ ). As the figure shows, the distributions are flat (within statistics) at low $\mathbb{E}_{T}$, which shows that the efficiency for QCD electron candidates to go from loose to tight $\left(\varepsilon_{Q C D}\right)$ is independent of $E_{T}$. We can thus determine $\varepsilon_{Q C D}$ from a constant fit within the region $E_{T}<10 \mathrm{GeV}$ (where the real electron contribution is negligible). The values found for the different jet multiplicities are given in Table 8.2. Within statistics, $\varepsilon_{Q C D}$ is independent of the jet multiplicity, indicating that the QCD fake electrons are independent of the topology of the event.

At this point, we can explain why we use the $\Delta \phi\left(\mathbb{E}_{T}, e\right)$ cut. Without this cut, which removes events where the $\not_{T}$ and the electron are in the same $\phi$ direction, these distributions are not flat at low $E_{T}$. This means that there is a contribution in the QCD background that is dependent on $\mathbb{E}_{T}$. Because we cannot estimate this dependence in the region where real electrons contribute $\left(\mathbb{E}_{T}>20 \mathrm{GeV}\right)$, we decided to accept the efficiency loss, which is given in Table 8.8, due to this cut.

$\varepsilon_{\text {sig }}$

The corresponding rate at which signal events are reduced from the loose to the tight sample, $\varepsilon_{s i g}$, is the efficiency for real electrons to pass the likelihood requirement. This was already measured in the $Z \rightarrow e e$ sample in Section 7.1.7. As $\varepsilon_{s i g}$ was found to be, within errors, constant as a function of jet multiplicity, we use the value obtained in the one jet multiplicity sample $\left(\varepsilon_{\text {sig }}=79.2 \pm 2.1 \%\right)$ for each of the four samples. 

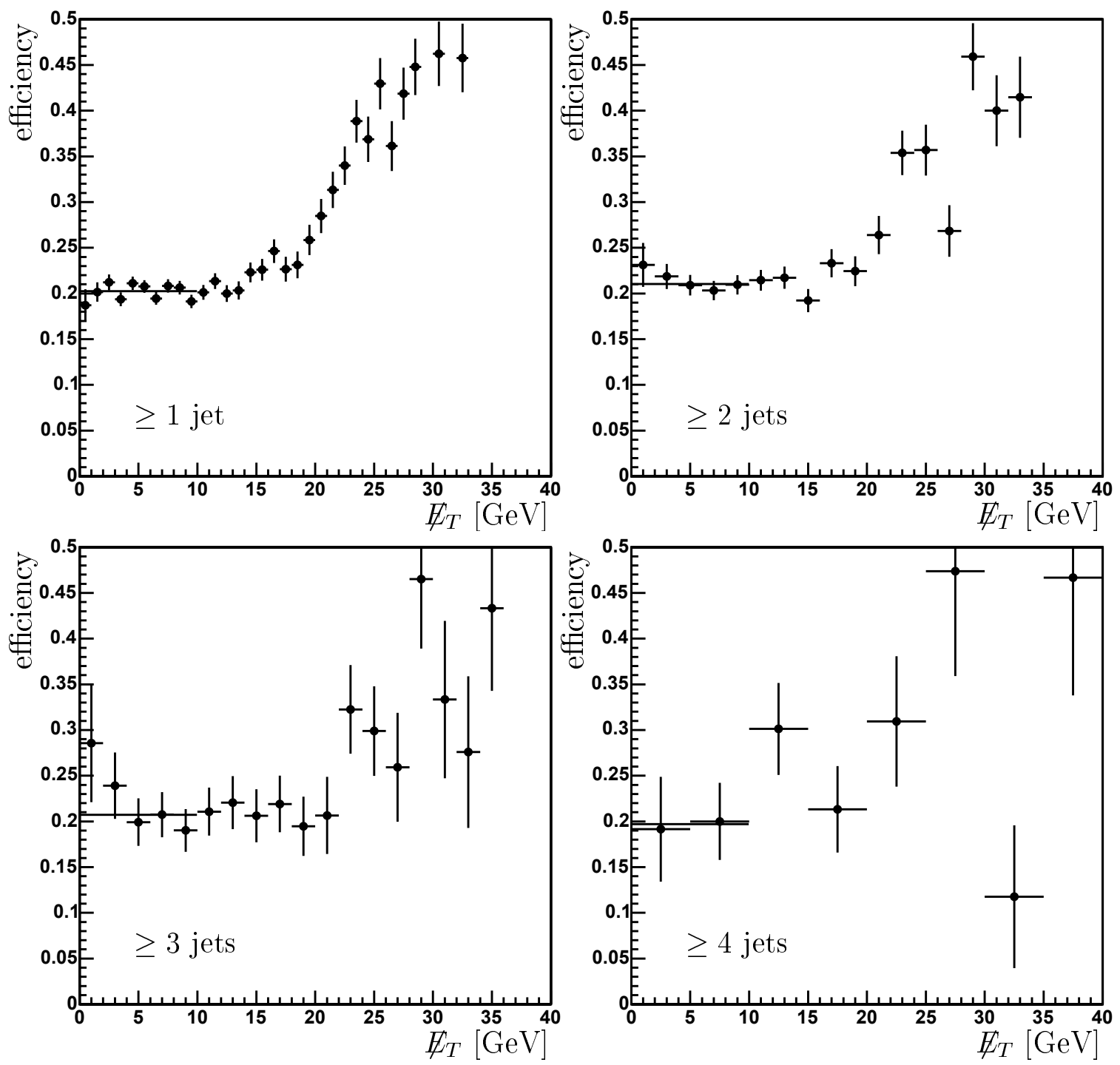

Figure 8.1: Efficiency for the electron to go from loose to tight as a function of $\mathbb{E}_{T}$ for jet multiplicities of $\geq 1, \geq 2, \geq 3$ and $\geq 4$.

\section{QCD background}

We now have all ingredients to estimate the QCD background. The number of events in the loose and the tight samples for each jet multiplicity are listed in Table 8.3, together with the estimate of the number of QCD and $W+$ jets events in the tight samples. The important number we will use later on is the QCD background in the four jet tight sample: $18.7 \pm 6.1$. As an additional check that the method is predicting the QCD background correctly, we plot the transverse mass of the electron/neutrino pair, and estimate the QCD background in each bin of transverse mass with the same method. 


\begin{tabular}{|c|c|c|c|c|c|c|}
\hline & $N_{\text {loose }}$ & $N_{\text {tight }}$ & $\begin{array}{l}N_{\text {tight }}^{\mathrm{QCD}} \\
\end{array}$ & $N_{\text {tight }}^{\mathrm{W}+\mathrm{t} \overline{\mathrm{t}}}$ & jet trigger eff. & $\begin{array}{l}N_{\text {tight, corr }}^{\mathrm{W}+\mathrm{t} \overline{\mathrm{t}}} \\
\end{array}$ \\
\hline$\geq 1$ jet & 8086 & 4195 & $759 \pm 47$ & $3436 \pm 96$ & $0.389 \pm 0.036$ & $8834 \pm 854$ \\
\hline$\geq 2$ jets & 3105 & 1426 & $373 \pm 27$ & 1053 & $0.669 \pm 0.027$ & $1573 \pm 106$ \\
\hline$\geq 3$ jets & 768 & 312 & $105 \pm 14$ & $207 \pm 27$ & $0.825 \pm 0.024$ & $251 \pm 33$ \\
\hline$\geq 4$ jets & 156 & 67 & $18.7 \pm 6.1$ & $48 \pm 12$ & $0.879 \pm 0.053$ & $55 \pm 14$ \\
\hline
\end{tabular}

Table 8.3: $N_{\text {loose }}, N_{\text {tight }}, N_{\text {tight }}^{\mathrm{QCD}}$ and $N_{\text {tight }}^{\mathrm{W}+\mathrm{t \overline {t }}}$ before and after jet trigger efficiency correction.

This is shown in Figure 8.2. In all but the four jet sample, we clearly see a contribution from the $W$ and a falling QCD contribution.

Let us discuss here the choice we have made to look only at events where the electron ends up in the central calorimeter $\left(\left|\eta_{D}\right|<1.1\right)$. The procedure to estimate the QCD background has also been applied to events where the electron ends up in the endcap calorimeter $\left(1.5<\left|\eta_{D}\right|<2.5\right)$. Although the efficiency for QCD electron candidates to go from loose to tight (without the $\mathbb{E}_{T}$ cut) is flat at low $\mathbb{E}_{T}$, there are two problems:

- $\varepsilon_{Q C D}$ depends on the jet multiplicity of the events for the EC, indicating an unwanted dependence on the topology of the event.

- The estimate of the $W+t \bar{t}$ contribution at low electron/neutrino transverse mass has a negative peak at a transverse mass of $20 \mathrm{GeV}$.

Both problems are shown in Figure 8.3.

These problems indicate that we don't have a correct description of the background in the EC. Moreover, because there is only a $19 \%$ relative gain in acceptance times reconstruction efficiency when including the EC and the background in the EC is much higher than in the CC, we decided to concentrate on the CC for this analysis.

\subsection{Berends scaling}

Now that we know the QCD and $W+t \bar{t}$ contributions in each of the four tight samples (distinguished by their number of jets), the next step is to estimate the number of $W$ events in the four jet sample, which we will use as our final sample. What we expect to see is that in the lower multiplicities, the $W+t \bar{t}$ events are dominated by $W$ 's, while in the four jet multiplicity there will be a significant contribution from $t \bar{t}$.

We will evaluate the $W$ background by using the Berends scaling rule $[117,118$, 

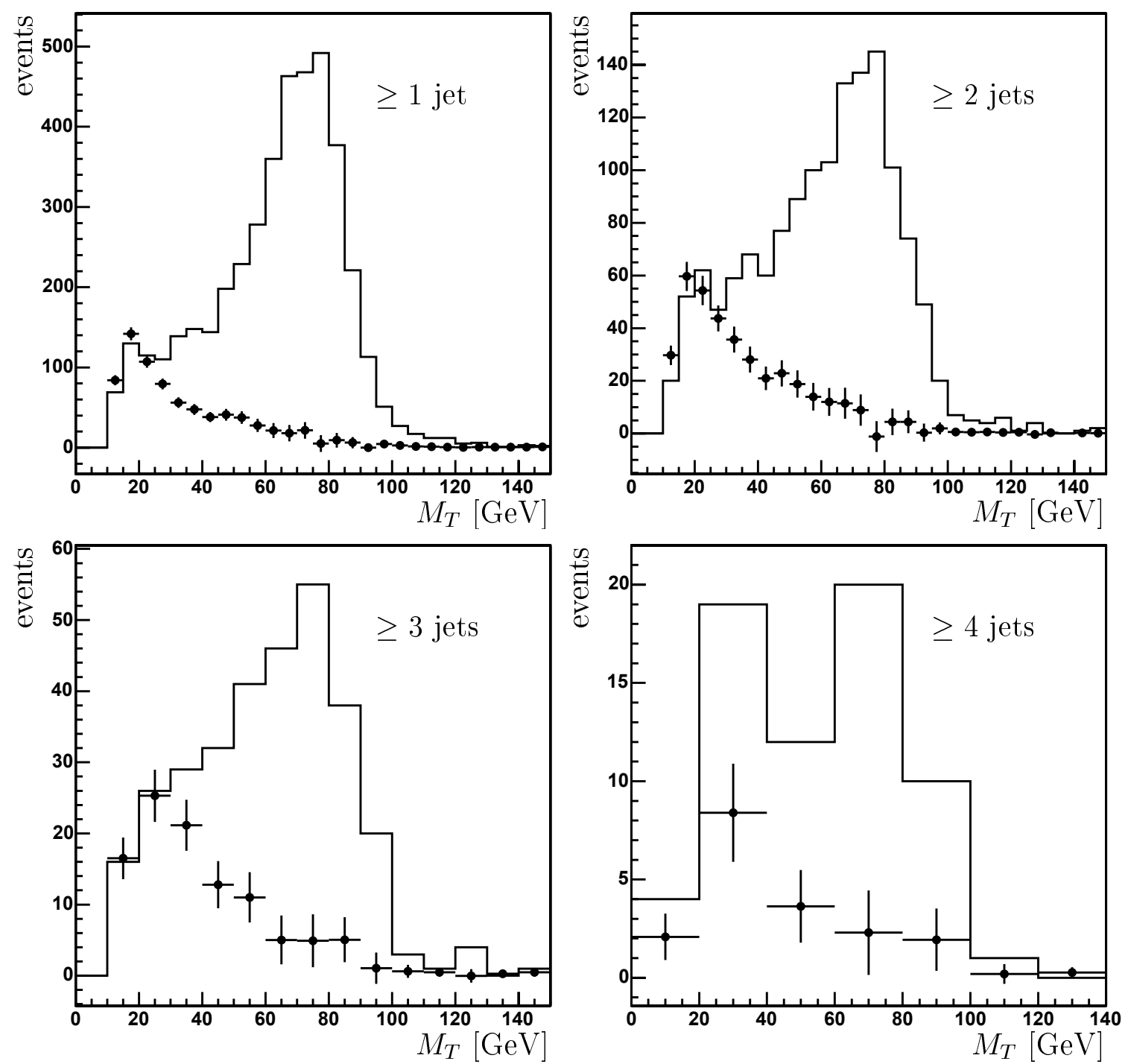

Figure 8.2: Transverse mass distributions for $\geq 1, \geq 2, \geq 3$, and $\geq 4$ jet multiplicity. Histograms are the observed numbers of events after selection cuts, points give the QCD background estimate.

119, 120], which reads

$$
\frac{\sigma\left(W+(n+1)_{\text {jets }}\right)}{\sigma\left(W+n_{\text {jets }}\right)}=\alpha,
$$

where $\alpha$ is dependent on the jet transverse energy and pseudo rapidity requirements, but independent of $n$, the inclusive jet multiplicity. Using this behavior (as a function of jet multiplicity) for the $W$ contribution to the $W+t \bar{t}$ samples, the total number of 


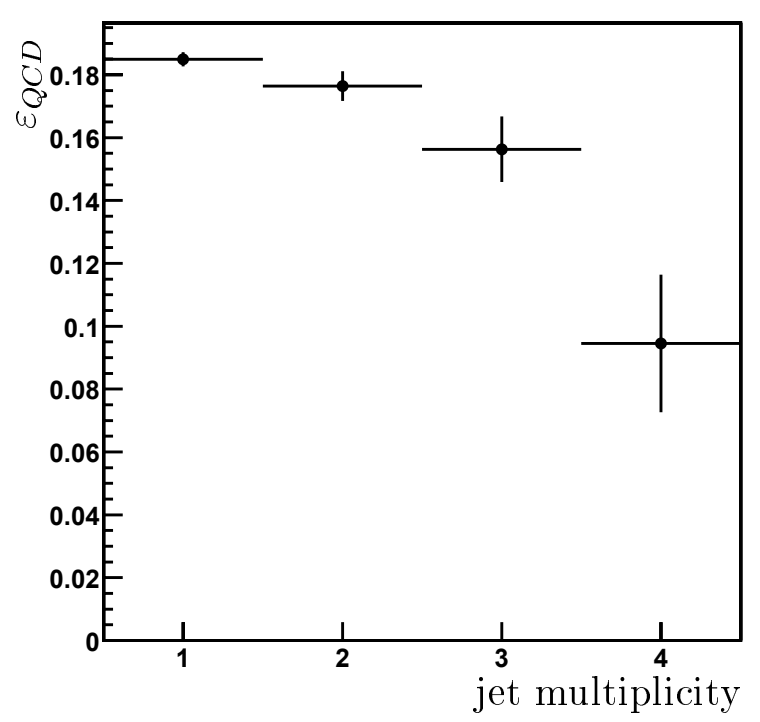

(a)

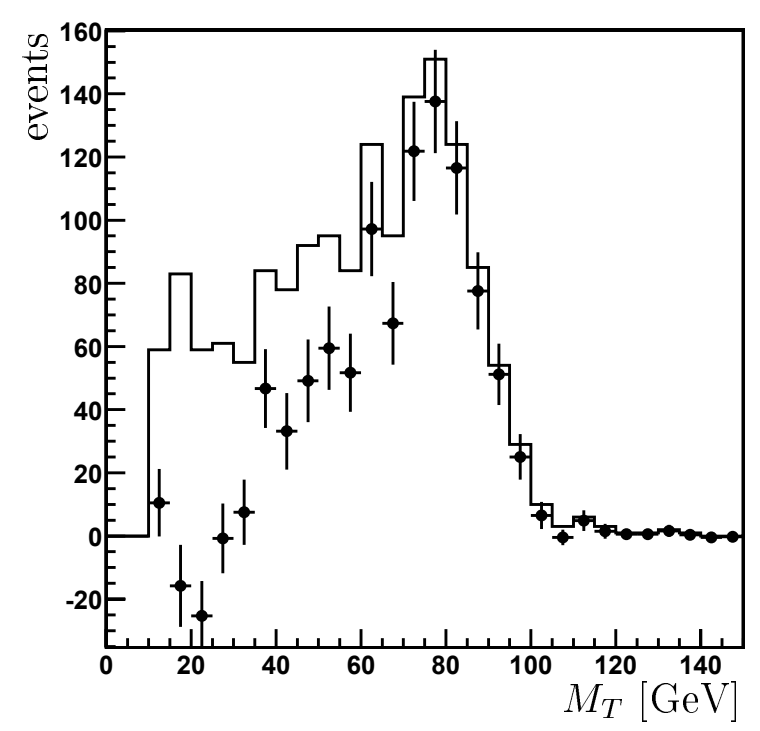

(b)

Figure 8.3: Figure (a) shows $\varepsilon_{Q C D}$ as a function of the jet multiplicity for events with the electron candidate in the EC. Figure (b) shows the transverse mass distribution for events with the electron candidate in the $E C$ which have a jet multiplicity of one. The histogram is the observed number of events, points give the $W+t \bar{t}$ signal estimate.

\begin{tabular}{c|c}
\hline \hline jet multiplicity $i$ & $f_{i}^{t \bar{t}}$ \\
\hline \hline 1 & 1.000 \\
\hline 2 & 0.992 \\
\hline 3 & 0.885 \\
\hline 4 & 0.545 \\
\hline \hline
\end{tabular}

Table 8.4: Inclusive top fraction as a function of jet multiplicity.

events in the different $W+t \bar{t}$ samples can be connected as follows:

$$
N_{i}^{\mathrm{W}+\mathrm{t} \overline{\mathrm{t}}}=N_{1}^{W} \times \alpha^{i-1}+f_{i}^{t \bar{t}} N^{t \bar{t}} .
$$

Here $N_{i}^{\mathrm{W}+\mathrm{t} \overline{\mathrm{t}}}$ is the number of events remaining with a jet multiplicity $\geq i$ in the $W+t \bar{t}$ sample, $N_{1}^{W}$ is the number of W events with at least one jet in the final state, and $f_{i}^{t \bar{t}}$ is the fraction of $t \bar{t}$ events expected to survive the criteria of a jet multiplicity greater or equal to $i$. These fractions are computed from Monte Carlo simulation, and are given in Table 8.4. The values of $\alpha, N_{1}^{W}$ and $N^{t \bar{t}}$ can be obtained from a fit to the distribution of $N_{i}^{\mathrm{W}+\mathrm{t} \overline{\mathrm{t}}}$ for $i=1,2,3,4$. Using the information from this fit, we can then 


\begin{tabular}{c|c}
\hline \hline$\alpha$ & $0.168 \pm 0.019$ \\
\hline$N_{1}^{W}$ & $9012 \pm 789$ \\
\hline$N^{t \bar{t}}$ & $15 \pm 39$ \\
\hline \hline
\end{tabular}

Table 8.5: Results of the Berends scaling fit.

extrapolate the number of $\mathrm{W}$ events in the four or more jets topology:

$$
N_{4}^{W}=N_{1}^{W} \times \alpha^{3}
$$

Before we can apply this to our samples, there is one additional effect we need to take into account: the inclusive jet multiplicity distribution for $W+t \bar{t}$ events is influenced by the presence of jet selection criteria in the trigger ${ }^{2}$, which is described in Section 6.3.2. This jet trigger efficiency is estimated from the fraction of $W+t \bar{t}$ events (after QCD background subtraction) that were triggered by a single electron trigger ${ }^{3}$, and in addition also fired the electron + jet trigger used in the analysis. As a cross check, the same number is estimated from the number of events in the tight sample (without QCD background subtraction) ${ }^{4}$. These efficiencies are measured and applied separately in each jet multiplicity sample. The jet trigger efficiencies we found are given in Table 8.3. Correcting the $N_{\text {tight }}^{\mathrm{W}+\mathrm{t \overline {t }}}$ for this effect, we get the numbers in the column $N_{\text {tight, corr }}^{\mathrm{W}+\mathrm{t} \overline{\mathrm{t}}}$. These are now corrected for the trigger requirement, and can thus be fit with (8.5). The number of events in each bin $\left(N_{\text {tight, corr }}^{\mathrm{W}+\mathrm{t} \overline{\mathrm{t}}}\right)$ and the contribution from $W$ events that we get from the fit are shown in Figure 8.4. Although there are correlations between the bins, we ignore them for the fit, because they are small (only a small amount of the events in each bin is also in the previous bin, because the spectrum is steeply falling). The small effect that this might have on the fit and its errors is thus neglected. The parameters extracted from the fit are given in Table 8.5.

Using (8.6), we get an estimate for the number of $W$ events in the four jet tight sample of $43.1 \pm 11.9$. But this is the estimate without the jet trigger efficiency. To get the actual number of $\mathrm{W}$ events in the four jet tight sample, we need to multiply this number by the jet trigger efficiency for events with a jet multiplicity $\geq 4$ (which is given in Table 8.3). This gives as the final estimate for the number of $W$ events in the four jet tight sample $37.8 \pm 10.7$.

\footnotetext{
${ }^{2}$ It is easier for several jets to fire the single jet trigger requirement than it is for one jet.

${ }^{3}$ Although it would simplify things to use a single electron trigger instead of an electron + jet trigger to do this analysis, this was not possible because the single electron trigger was prescaled during high luminosity runs.

${ }^{4}$ We expect to find the same efficiency here, because the QCD contribution is relatively small, and the jets in $W+$ jets and QCD events are expected to have roughly the same $\eta$ and $p_{T}$ distributions.
} 


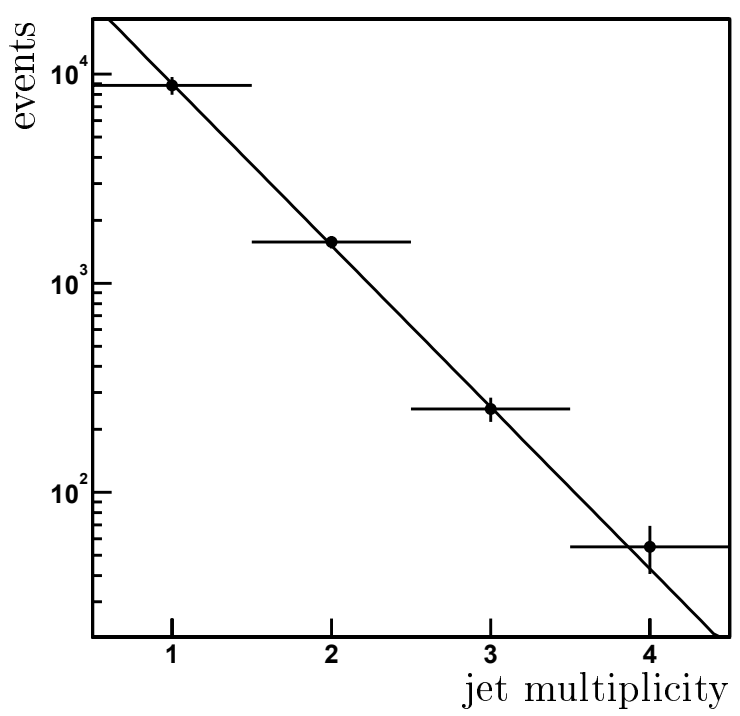

Figure 8.4: The points show the inclusive number of $W+$ jets and $t \bar{t}$ events. The line gives the contribution from $W+$ jets events that we get from the Berends scaling fit.

\subsection{Topological selection}

In the Berends scaling fit, there seems to be a contribution from the $t \bar{t}$ events, but it is hardly significant. We therefore need to enhance the contribution of signal in this sample. We do this by applying topological cuts. We select variables that provide good separation between signal and background. The two most discriminating variables used in Run I [102] in the lepton plus jets analyses are the scalar sum over all jet transverse energies $H_{T}=\sum_{\text {jets }} E_{T}$ and the aplanarity $\mathcal{A}$, a measure of the flatness of the event, defined as $3 / 2$ times the smallest eigenvalue of the normalized momentum tensor $\mathcal{M}$ :

$$
\mathcal{M}_{i j}=\frac{\sum_{o} p_{i}^{o} p_{j}^{o}}{\sum_{o}\left|\overrightarrow{p^{o}}\right|^{2}}
$$

where $\overrightarrow{p^{o}}$ is the momentum of a reconstructed object $o, i$ and $j$ are the laboratory spatial coordinates, and the objects included in the sum are the jets and the reconstructed leptonic ${ }^{5} W(\mathcal{A}(W+$ jets $))$. Large values of $\mathcal{A}$ are indicative of spherical events, whereas small values correspond to more planar events. $t \bar{t}$ events are quite spherical, as is typical for the decay of a heavy object. $W$ +jets and QCD events are more planar, owing

\footnotetext{
${ }^{5}$ The $W$ is reconstructed using the electron momentum and the missing transverse energy. Constraining the electron/neutrino invariant mass to the $W$ mass leads in general to two solutions for the $p_{z}$ of the neutrino. The solution with the smallest $\left|p_{z}\right|$ is chosen. If the electron/neutrino transverse mass is higher than the $W$ mass, the missing transverse momentum is scaled so that the transverse mass is equal to the $W$ mass, and $p_{z}$ is thus taken to be zero.
} 


\begin{tabular}{c|c|c|c|c|c}
\hline \hline & $\mathrm{N}_{\text {obs }}$ & $\mathrm{N}_{b k g}$ & $\mathrm{~N}_{W}$ & $\mathrm{~N}_{Q C D}$ & $\varepsilon_{t \bar{t}}(\%)$ \\
\hline \hline preselection $\left(\mathrm{N}_{\text {jets }} \geq 4\right)$ & 67 & 56.6 & 37.8 & 18.7 & 100.0 \\
\hline$\left|\eta_{W}\right|<2$ & 64 & 54.9 & 36.9 & 18.0 & 98.9 \\
\hline$E_{T}^{W}>60 \mathrm{GeV}$ & 55 & 48.4 & 35.2 & 13.2 & 97.3 \\
\hline$H_{T}>180 \mathrm{GeV}$ & 27 & 21.6 & 16.6 & 5.0 & 82.7 \\
\hline $\mathcal{A}(W+$ jets $)>0.065$ & 12 & $7.2 \pm 1.7$ & $5.3 \pm 1.6$ & $1.9 \pm 0.7$ & 52.9 \\
\hline \hline
\end{tabular}

Table 8.6: Number of observed events, estimated background and relative signal efficiency after each of the topological cuts.

primarily to the fact that the jets in these events arise mainly from gluon radiation. Another variable used is $E_{T}(W)=p_{T}(e)+E_{T}$ (scalar sum). Only jets that have $p_{T}>15 \mathrm{GeV}$ and $\left|\eta_{j e t}\right|<2.5$ are used in calculating these topological variables.

In addition, a cut on $\eta_{W}$ is applied. This is the pseudorapidity of the reconstructed leptonic $W$. This cut is designed to remove from consideration those regions of phase space where the $W+$ jets Monte Carlo does not model the $W+$ jets data very well (see [102]). Although in the reference the data is compared with the VECBOS [119] Monte Carlo, the ALPGEN Monte Carlo we use has the same underlying matrix elements, and will therefore have the same problem.

The efficiency for the topological selection is determined from $\mathrm{MC}$ for the $t \bar{t}$ signal and the $W$ +jets background. The efficiency for QCD is measured in data, using a sample containing an EM cluster plus four jets, with identical selection cuts as the loose four jet sample, except that no electron identification cuts are applied. This sample will thus be dominated by QCD.

An indication of the effect of different cuts on $H_{T}$ and aplanarity is shown in Figure 8.5. While the cuts used in this analysis $\left(H_{T}>180 \mathrm{GeV}\right.$ and $\left.\mathcal{A}>0.065\right)$ do not give the optimal statistical uncertainty on the cross section (equivalent to optimal $\left.\frac{\sqrt{s+b}}{s}\right)$, for a given amount of background, they are rather good 6 .

The efficiency of the chosen topological cuts for W+jets and QCD are 14.1 $\pm 1.1 \%$, and $10.0 \pm 1.6 \%$, respectively. The number of observed events, the predicted background and the relative signal efficiency after each of the topological cuts are listed in Table 8.6. The 12 candidate events we are left with are listed in Table 8.7. Figure 8.6 shows one of the candidate events.

\footnotetext{
${ }^{6}$ The optimal set of cuts (as indicated in the figure) was not used, because the optimization study is not complete (it should also include the effects of the systematic error). In addition, the expected background before topological cuts was set to the value that we found in the data sample, which biases the cut selection. A full study would include both statistical and systematic errors, and would use an estimate of the background which is independent of the used sample. We therefore decided to use the Run I cuts.
} 


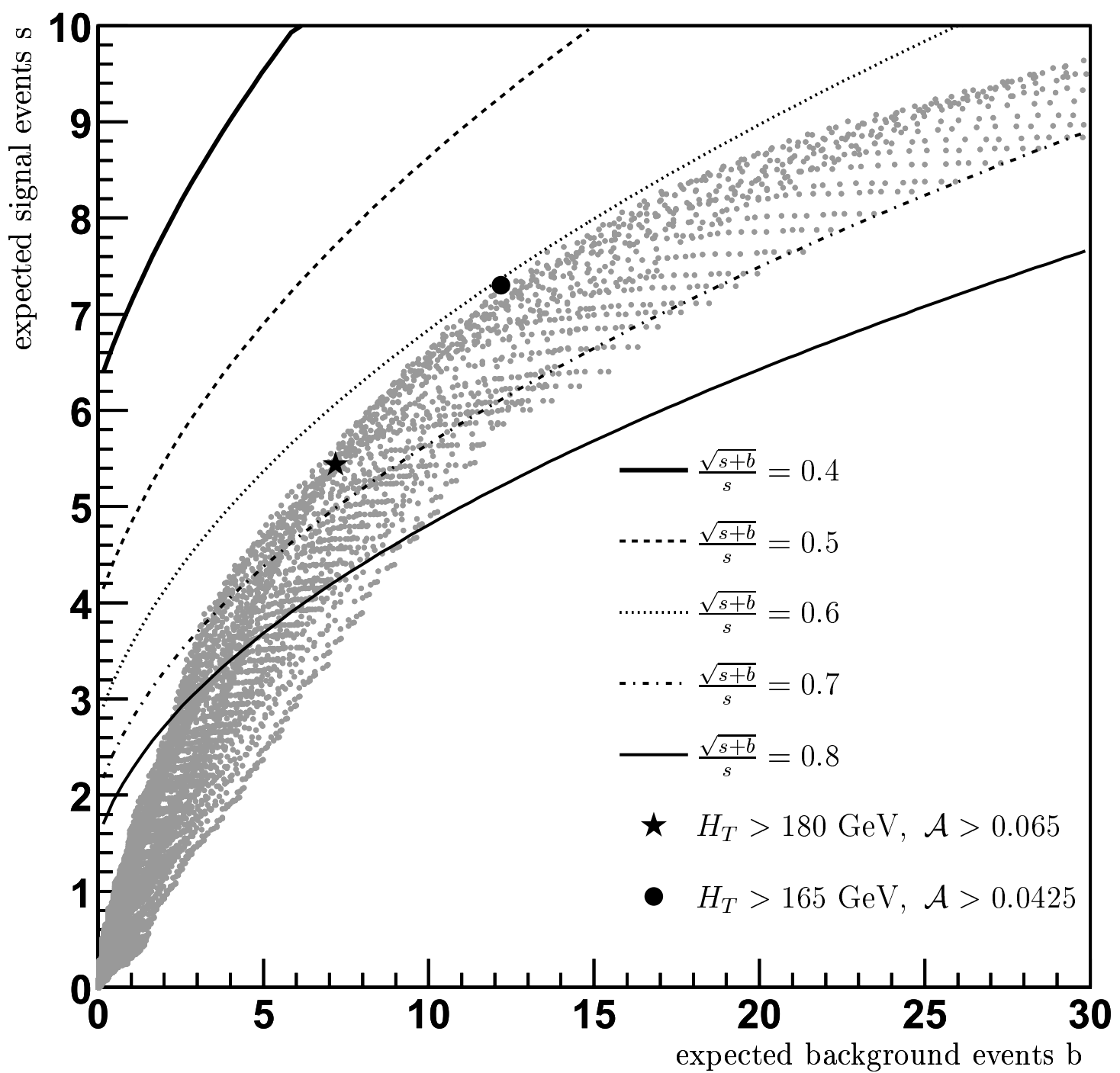

Figure 8.5: Expected signal versus expected background, where each point corresponds to a different combination of $\mathcal{A}$ and $H_{T}$ cuts. Expected signal is the number of events we expect for an integrated luminosity of $92 \mathrm{pb}^{-1}$ (taking a signal cross section of 7 pb). Expected background gives the number of background events in our sample that would survive the topological cuts under study. The cut pair chosen for this analysis is indicated by the star. The circle shows the "optimal" cut point, if only the statistical error is taken into account $\left(\frac{\sqrt{s+b}}{s}\right.$ is a measure for the (statistical) uncertainty on the cross section $\frac{\delta \sigma}{\sigma}$ ). The curves are contours of constant (statistical) uncertainty on the cross section. 


\begin{tabular}{c|r}
\hline \hline run & event \\
\hline \hline 164041 & 21154890 \\
\hline 165600 & 23337177 \\
\hline 166830 & 17709325 \\
\hline 167885 & 19738570 \\
\hline 169923 & 16396718 \\
\hline 173076 & 3125163 \\
\hline 174666 & 17387772 \\
\hline 175335 & 29822900 \\
\hline 175818 & 1123080 \\
\hline 177034 & 11407190 \\
\hline 177275 & 5302321 \\
\hline 178152 & 32384663 \\
\hline \hline
\end{tabular}

Table 8.7: Candidate events.

\subsection{Alternative $W+$ jets background prediction}

There is an alternate method to estimate the $\mathrm{W}+$ jets background, which is independent of Berends scaling and the jet trigger efficiency. This uses the same principle as used for estimating the QCD background, except the loose and the tight sample are now replaced by the tight and the topological sample (the events that pass the topological cuts).

The number of events in the tight sample $\left(N_{\text {tight }}\right)$ and the number of events in the topological sample $\left(N_{\text {topo }}\right)$ can be written as:

$$
\begin{aligned}
N_{\text {tight }} & =N^{t \bar{t}}+N^{W}+N^{Q C D} \\
N_{\text {topo }} & =\varepsilon_{\text {topo }}^{t \bar{t}} N^{t \bar{t}}+\varepsilon_{\text {topo }}^{W} N^{W}+\varepsilon_{\text {topo }}^{Q C D} N^{Q C D} \\
& \equiv N_{\text {topo }}^{t \bar{t}}+N_{\text {topo }}^{W}+N_{\text {topo }}^{Q C D} .
\end{aligned}
$$

We now solve for the number of $W$ events in the topological sample $\left(N_{\text {topo }}^{W}\right)$ :

$$
N_{\text {topo }}^{W}=\frac{\varepsilon_{\text {topo }}^{W}}{\varepsilon_{\text {topo }}^{W}-\varepsilon_{\text {topo }}^{t \bar{t}}}\left\{N_{\text {topo }}-\varepsilon_{\text {topo }}^{t \bar{t}} N_{\text {tight }}-\left(\varepsilon_{\text {topo }}^{Q C D}-\varepsilon_{\text {topo }}^{t \bar{t}}\right) N^{Q C D}\right\} .
$$

This method gives an estimate of the $\mathrm{W}+$ jets background after topological cuts of $5.6 \pm 1.1$ events, where the error is statistical only. This is consistent with the number we find using Berends scaling. We will therefore use the latter number in the rest of this analysis. 


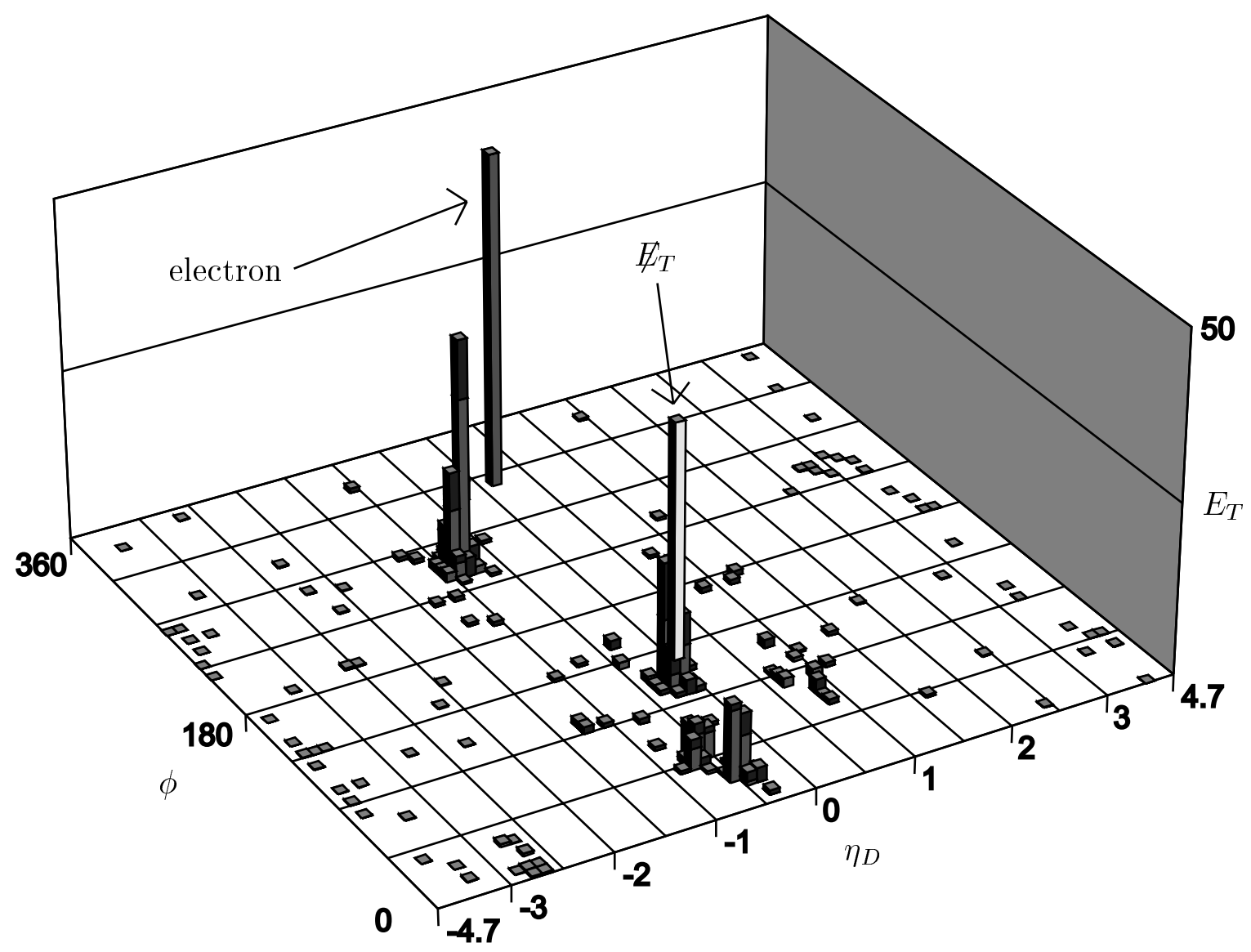

Figure 8.6: Event display of a candidate event (run 174666, event 17387772). The figure shows the distribution of transverse energy in the calorimeter as a function of $\eta_{D}$ and $\phi$. The transverse energy deposited by the electron and the $\mathbb{E}_{T}$ resulting from all energy deposits are indicated by arrows. The rest of the energy is reconstructed into four jets.

\subsection{Selection efficiency}

Now that we have estimated the background in the final sample, and thus have an estimate for the number of top events we see in the final sample, we will turn our attention to explaining the cuts (where needed) and calculating the efficiency of each cut. The selection cuts and the efficiency of each cut are listed in Table 8.8, for a top mass of $175 \mathrm{GeV}$. The sensitivity of these cuts to the top mass will be treated as a systematic uncertainty (see next section).

The electron selection requires that we find the electron, that it ends up in the Central Calorimeter $\left(\left|\eta_{D}\right|<1.1\right)$, passes calorimeter cluster and likelihood requirements, and has a $p_{T}$ greater than $20 \mathrm{GeV}$. All these requirements on the electron are simply to 


\begin{tabular}{c|c|c|c}
\hline \hline Selection cut & $\varepsilon$ & \multicolumn{2}{|c}{ derived from } \\
\hline \hline second electron veto & & MC & data \\
\hline acceptance $\times$ reconstruction & $60.6 \pm 0.8 \%$ & $\sqrt{ }$ & $\mathrm{c}$ \\
\hline$p_{T}(e)>20 \mathrm{GeV}$ & $92.0 \pm 0.4 \%$ & $\sqrt{ }$ & \\
\hline cluster selection electron & $82.9 \pm 0.7 \pm 2.9 \%$ & $\mathrm{c}$ & $\sqrt{ }$ \\
\hline likelihood electron & $68.1 \pm 0.8 \pm 2.9 \%$ & $\mathrm{c}$ & $\sqrt{ }$ \\
\hline$E_{T}>20$ GeV & $89.5 \pm 0.5 \%$ & $\sqrt{ }$ & \\
\hline soft muon veto & $81.6 \pm 0.8 \%$ & $\sqrt{ }$ & $\mathrm{c}$ \\
\hline$\Delta \phi\left(\not_{T}, e\right)>0.5$ & $87.9 \pm 0.6 \%$ & $\sqrt{ }$ & \\
\hline$N_{\text {jets }} \geq 4\left(|\eta|<2.5\right.$ and $\left.p_{T}>15 \mathrm{GeV}\right)$ & $54.5 \pm 1.1 \%$ & $\sqrt{ }$ & \\
\hline topological cuts & $52.9 \pm 1.5 \%$ & $\sqrt{ }$ & \\
\hline primary vertex & $96.7 \pm 1.9 \%$ & & $\sqrt{ }$ \\
\hline trigger efficiency & $92 \pm 1 \pm 2 \%$ & $\sqrt{ }$ & $\sqrt{ }$ \\
\hline$\tau$ factor & $\times 1.04$ & $\sqrt{ }$ & \\
\hline NLO factor & $\times 1.08$ & $\sqrt{ }$ & \\
\hline \hline total signal efficiency & $5.8 \pm 0.2$ stat $\%$ & & \\
\hline \hline
\end{tabular}

Table 8.8: Selection cuts efficiencies for a top mass of $175 \mathrm{GeV}$. The "derived from" column lists if the number are derived from data and/or $M C(\sqrt{ })$, and if correction factors are applied, where these were derived (c). An explanation can be found in the main text. 
reduce background. The second electron veto (no second electron with $p_{T}>15 \mathrm{GeV}$ and $\left.\left|\eta_{D}\right|<2.5\right)$ is made to keep the analysis orthogonal to the di-electron $t \bar{t}$ analysis and to reject $Z+$ jets background.

The acceptance and efficiencies for the reconstruction, kinematic cut and second electron veto are determined from a MC $t \bar{t}$ sample. For the reconstruction efficiency, a correction factor of 0.967 is applied to account for the difference in electron reconstruction efficiency seen between data and MC, see Section 7.1.6.

The calorimeter cluster selection efficiency is measured in $Z \rightarrow e e$ data, see Section 7.1.7. To account for the difference in topology and particle multiplicities in the $Z \rightarrow e e$ events (where the efficiency is measured) and the $t \bar{t}$ events (where it is used), a correction factor of 0.946 is applied to the efficiency as measured in $Z \rightarrow e e$ data. This correction factor was derived by looking at the difference between the electron cluster selection efficiency in $Z \rightarrow e e \mathrm{MC}(97.5 \pm 0.2 \pm 0.8 \%)$ and in $t \bar{t} \mathrm{MC}(92.2 \pm 0.4 \%)$. The same method was used to get the final electron likelihood efficiency. For the likelihood, we get a correction factor of 0.943 .

The missing transverse energy cut is applied to reduce the huge QCD background, which can basically only have $E_{T}$ due to imperfect energy measurements of the jets (the resolution of the energy measurement is not zero).

The soft muon veto is applied to keep this measurement orthogonal to the measurement which uses soft muon tagging. The requirement is that events are vetoed when there is a muon (with $p_{T}>4 \mathrm{GeV}$ ) within a jet. When we measure the efficiency for the soft muon veto in Monte Carlo, we get $83.3 \pm 0.7 \%$, meaning that in $16.7 \pm 0.7 \%$ of the events one or more jets are tagged by a soft muon. But it has been shown [121] that the muon reconstruction efficiency is a factor $1.11 \pm 0.05$ higher in data than in MC. Thus in data, we expect the efficiency for an event to be tagged to be $18.4 \pm 0.8 \%$. Therefore we will use a soft muon veto efficiency of $81.6 \pm 0.8 \%$.

The reason for the $\Delta \phi\left(E_{T}, e\right)$ cut has already been explained in Section 8.3. The jet multiplicity and the topological cuts are applied to reduce the background, both QCD and $W+$ jets. All these efficiencies are taken from MC.

Next are the primary vertex cut and the trigger requirement. We require a good primary vertex because we need it to define the DCA of the electron (which is used in the electron likelihood) and because it reduces the chance of events showing up in the sample that only have energy deposits in the calorimeter due to noise. The efficiency of the primary vertex requirement is measured in the four jet tight data sample. The trigger requirement is last on the list, although it is of course applied first. It is put at the end of the list as a reminder that the trigger efficiency is measured for events that pass all requirements, including object identification and topological cuts. How this efficiency was derived has already been explained in Section 6.3.4.

The " $\tau$ factor" takes into account that instead of the $W$ (coming from one of the tops) decaying to an electron, it can decay to a tau. If the tau decays to an electron, 
this gives a contribution to the efficiency. As the branching ratio times efficiency for this to happen is $4 \%$ of the direct decay to an electron, we take this into account by multiplying the efficiency by 1.04. This is lower than the branching ratio for the tau to decay to an electron (18\%), because the momentum of the electron is lower as a result of the cascade decay.

All the efficiencies so far have been derived with a MC that has a Leading Order description of the $t \bar{t}$ production process. Recently, a MC generator (MC@NLO [108]) has become available that computes the $t \bar{t}$ production process at Next to Leading Order. The program uses HERWIG [14] to decay the top quarks and to simulate the (properly matched) parton shower. We do not use it to get the signal efficiency directly, but use it to derive a correction factor. This correction factor is derived by calculating the difference in signal efficiency on a $t \bar{t}$ MC sample generated with MC@NLO, and a $t \bar{t}$ MC sample generated with standard HERWIG. The only difference between the two samples is thus the treatment of the hard scattering process (NLO and LO respectively). The correction factor we find is $1.08 \pm 0.05$, meaning that NLO gives a $8 \%$ higher signal selection efficiency. As the NLO prediction is expected to be better than the LO one, we can directly apply this correction factor to our signal efficiency (which was derived with a LO description of the $t \bar{t}$ production process $)^{7}$.

Combining all the selection efficiencies, we get a total $t \bar{t}$ signal efficiency of $5.8 \pm$ $0.2 \%$.

\subsection{Systematic uncertainties}

In this section we will discuss the individual uncertainties which affect the background and the signal efficiencies. A summary of the different contributions is given in Table 8.9 .

\section{Luminosity}

The uncertainty on the luminosity measurement is $10 \%$ and comes from the uncertainty in the total $p \bar{p}$ inelastic cross section and incomplete modelling of the luminosity monitors acceptance [122].

\section{Jet energy scale}

The uncertainty in the jet energy scale affects the cross section determination, but only through the uncertainty of the relative scale between data and MC. We take the MC and data uncertainties to be uncorrelated with each other, thus the uncertainty on the

\footnotetext{
${ }^{7}$ The uncertainty on the correction factor is treated as a separate systematic uncertainty, and is therefore not included in the statistical error on the selection efficiency.
} 


\begin{tabular}{c|c|c|c|c|c}
\hline \hline Effect & $\varepsilon$ & $N_{W}$ & $N_{Q C D}$ & $\mathcal{L}$ & $B r$ \\
\hline \hline Luminosity & & & & $\pm 10 \%$ & \\
\hline Jet energy scale (correlated) & $\begin{array}{c}+12 \% \\
{ }_{-14 \%} \\
\text { Jet energy scale (uncorrelated) }\end{array}$ & $\begin{array}{c}+3 \% \\
+6 \%\end{array}$ & & & \\
\hline Jet resolution & ${ }_{-8.3 \%}^{+4.0 \%}$ & $-16 \%$ & & & \\
\hline Jet ID & $\pm 3.2 \%$ & & & & \\
\hline$e$ resolution & $\pm 0.6 \%$ & & & & \\
\hline$e$ electron reconstruction & $\pm 2 \%$ & & & & \\
\hline$e$ cluster identification & $\pm 3.5 \%$ & & & & \\
\hline$e$ likelihood & $\pm 4.3 \%$ & & & & \\
\hline Trigger & $\pm 2.2 \%$ & & & & \\
\hline Primary vertex & $\pm 0.8 \%$ & & & & \\
\hline Top mass & ${ }_{-5.0 \%}^{+4.4 \%}$ & & & & \\
\hline Skimming & $\pm 0.2 \%$ & & & & \\
\hline Background estimate & & \pm 1.7 events & & \\
\hline MC statistical uncertainty & $\pm 3.3 \%$ & & & & \\
\hline Branching fraction & & & & & $\pm 1.6 \%$ \\
\hline Soft muon veto & $\pm 1.2 \%$ & & & & \\
\hline NLO/LO correction & $\pm 5 \%$ & & & & \\
\hline \hline
\end{tabular}

Table 8.9: Summary of systematic uncertainties. For each effect, the uncertainties on the signal efficiency, backgrounds ( $N_{W}$ and $\left.N_{Q C D}\right)$, integrated luminosity and branching fraction are given (where appropriate). 
scale between data and $\mathrm{MC}$ is the MC and data jet energy scale uncertainties added in quadrature.

The uncertainties on the JES are dominated by the uncertainty on the showering correction [114]. This is because the method used to derive this correction (showering profiles) has been shown to be only approximate, and thus large systematic errors are assigned to the correction. This means that a large part of the uncertainty on the JES is correlated between the jets. We therefore deal with the correlations between the jets as follows:

- We take the JES to be fully correlated between the different jets. We thus vary the energy of each jet in the event up or down by one sigma (of the MC and data uncertainties added in quadrature), for all jets in the event at the same time. This has an effect on both the signal selection efficiency $\left({ }_{-14 \%}^{+12 \%}\right)$ and the $\mathrm{W}+\mathrm{jets}$ topological efficiency and thus $N_{W}\left(\begin{array}{l}+3 \% \\ -6 \%\end{array}\right)$.

- In the second method, we take the JES as uncorrelated between the jets. We vary the energy of each jet by a randomly chosen amount from a Gaussian distribution with the width equal to the MC and data uncertainties added in quadrature, for each jet in the event independently. Again, both the signal efficiency $(+1 \%)$ and $N_{W}(+16 \%)$ are affected.

For the final systematic uncertainty due to the JES, we try both methods, and use the largest uncertainty (both up and down) that we get.

\section{Jet resolution}

As mentioned in Section 7.2.2, the jet energy resolution is different in data and MC. We therefore smear the MC such that the jet resolution in MC corresponds to the one measured in data. Of course, both the measured resolutions in data and $\mathrm{MC}$ have errors. By varying the smearing within these errors, we derive the systematic uncertainty on the signal selection efficiency due to the uncertainty in the jet resolution. This uncertainty on the selection efficiency is ${ }_{-8.3 \%}^{+4.0 \%}$. Of course, there is also an effect on the $\mathrm{W}+$ jets topological efficiency of $\underset{+16.5 \%}{-1.7 \%}$ (fully correlated with the effect on $t \bar{t}$ ).

\section{Jet identification}

We use the jet identification efficiency derived from the $t \bar{t} \mathrm{MC}$ sample. We treat the difference in efficiency between MC and data (see Section 7.2.4) as a systematic uncertainty. To be conservative, we take the largest discrepancy (in the central calorimeter) of $0.8 \%$ as the systematic uncertainty per jet. This gives a systematic uncertainty of $\pm 3.2 \%$ ( 4 jets) on the signal efficiency. 


\section{Electron resolution}

The electron $p_{T}$ resolution is different in data and MC. We smear all MC electrons by $4.7 \%$ in energy to make the resolution of MC electrons consistent with what was measured in data [123]. As systematic uncertainty due to electron resolution we take the full difference between smeared and unsmeared signal selection efficiency. This gives an uncertainty in the selection efficiency of $\pm 0.6 \%$.

\section{Electron reconstruction}

As explained in Section 7.1.6, there is a $2 \%$ systematic uncertainty in the 0.967 correction factor that is applied to the MC reconstruction efficiency to account for the differences seen in the reconstruction efficiency between data and MC. This leads to a $2 \%$ uncertainty in the signal selection efficiency.

\section{Electron identification}

Both the calorimeter cluster selection cuts and the likelihood give two contributions to the cross section uncertainty. The two contributions are:

- The systematic uncertainty of the measured efficiency in the $Z \rightarrow e e$ data sample, which comes from the uncertainty in the background estimation in those samples.

- The uncertainty on the correction factor that is applied to the efficiency measured in the $Z \rightarrow e e$ sample. This correction factor takes into account the difference in the $Z \rightarrow e e$ sample (where the efficiency is measured), and the $t \bar{t}$ sample (where it is used). It is derived by comparing the measured efficiency in $Z \rightarrow e e$ and $t \bar{t}$ MC. We quote half the effect of this correction factor as its systematic uncertainty.

Combining these two contributions to the systematic uncertainty, we get a systematic uncertainty of $\pm 3.5 \%$ due to the cluster selection, and $\pm 4.3 \%$ coming from the uncertainty on the likelihood efficiency.

\section{Trigger}

In Section 6.3.4 it is explained how the assumption of factorization of the trigger terms leads to a systematic uncertainty on the trigger efficiency. This gives a systematic uncertainty on the selection efficiency of $\pm 2.2 \%$. 


\section{Primary vertex}

The efficiency for finding a good primary vertex is measured in the four jet tight signal sample, which is dominated by $W+$ jet events. This efficiency might not be the same for $t \bar{t}$ and $W+$ jet events. We compare the primary vertex efficiency for these two samples in MC. For $t \bar{t}$, we find $99.4 \pm 0.2 \%$ and for $W+$ jets, we get $98.6 \pm 0.4 \%$. Thus the uncertainty on the selection efficiency due to the primary vertex requirement is $0.8 \%$.

\section{Top mass}

For this analysis, we have used a top mass of $175 \mathrm{GeV}$. The signal selection efficiency depends on the top mass. Therefore the uncertainty in the top mass (about $5 \mathrm{GeV}$ ) gives an uncertainty in the selection efficiency. To derive this uncertainty, we calculated the selection efficiency for various MC samples with different top masses. We fit the efficiency as a function of mass (see Figure 8.7) to lower the dependence on the statistical fluctuations. We get a (relative) uncertainty on the selection efficiency of ${ }_{-5.0 \%}^{+4.4 \%}$ when we vary the top mass by $5 \mathrm{GeV}$.

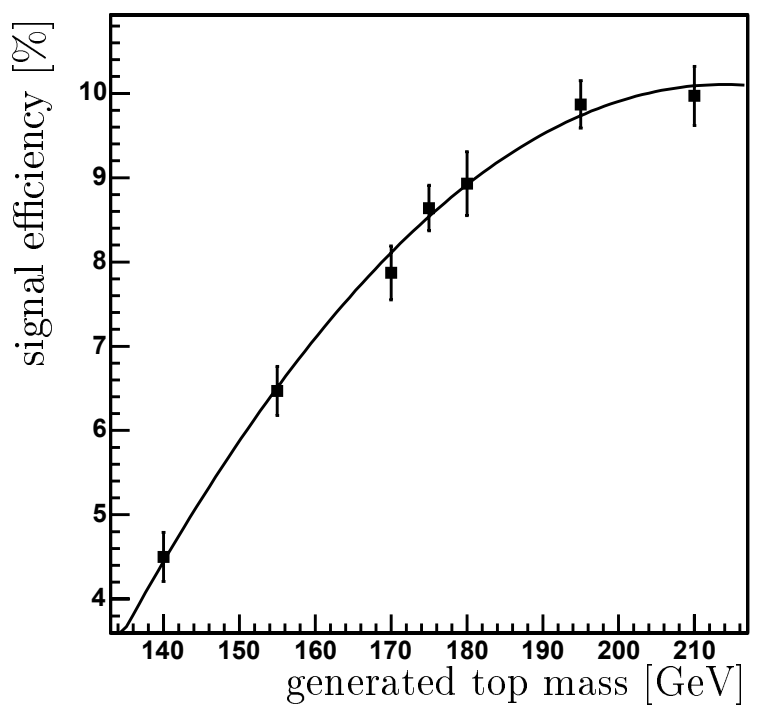

Figure 8.7: Signal selection efficiency as a function of generated top mass.

\section{Skimming}

Before the full analysis code is used, a sample is skimmed (preselected) based on simple properties. Some events might have passed the selection criteria in the analysis code that may not have passed the skimming, because: 
- The skimming requires that there is a $10 \mathrm{GeV}$ track near the electron. In some extreme cases the track of the electron might be reconstructed below $10 \mathrm{GeV}$.

- The version of the electron reconstruction that is used for the skimming and the one in the analysis code are slightly different.

- During most of the run period from which data is used for this analysis, there were problems in the calorimeter electronics. As a result of this, part of the energy deposited in certain towers would also show up in neighboring towers. These energy sharing problems are corrected in the analysis. At the skimming stage these problems were, however, still present.

The size of these effects has been estimated by simulating them on a $t \bar{t}$ Monte Carlo sample, leading to an uncertainty on the signal selection efficiency of $\pm 0.2 \%$ for the three effects together.

\section{Uncertainty on background estimate}

There is an uncertainty on the background estimate:

- For the QCD background, this comes from the uncertainties on the input parameters for the matrix method and the statistical uncertainty on the topological cut efficiency.

- For the $\mathrm{W}+$ jets background, it comes from the uncertainty on the Berends scaling fit (which in turn depends on the uncertainties in the matrix method and jet trigger efficiencies) and again the uncertainty on the topological cut efficiency.

\section{Statistical uncertainty on signal efficiency}

There is a statistical uncertainty on the signal efficiency determination because of the limited statistics available in the $Z \rightarrow e e$ data sample and the Monte Carlo samples. This leads to a systematic uncertainty in the signal efficiency.

\section{Branching ratio}

We use $(10.68 \pm 0.12) \%$ for the $W \rightarrow l \nu$ branching fraction, and $(67.96 \pm 0.35) \%$ for the $W \rightarrow$ hadrons branching fraction [103]. This gives a $t \bar{t} \rightarrow e+$ jets branching fraction of 0.145 with a relative uncertainty of $1.6 \%$. 


\section{Soft muon veto}

We calculated the soft muon veto efficiency by applying a $1.11 \pm 0.05$ correction factor to the soft muon tag efficiency as measured in Monte Carlo, because the muon reconstruction efficiency is higher in data than in Monte Carlo. The uncertainty on this correction factor gives a systematic uncertainty on the soft muon veto efficiency, and thus on the selection efficiency. The relative uncertainty on the selection efficiency from the soft muon veto is thus $1.2 \%$.

\section{Berends scaling}

The assumption of Berends scaling is tested by looking at $Z \rightarrow e e$ events. We require that both electrons in these events pass the same cuts as used in signal sample of the analysis. We use the number of events with a minimum jet multiplicity of one and two to predict the number of events with a jet multiplicity of three. The exponential fit to the first two jet multiplicities is shown in Figure 8.8(a). The fit predicts $9.7 Z \rightarrow e e$ events with a minimum jet multiplicity of three, while the actual number of events found is 9 . In addition we fit the number of events in the first three multiplicity bins,

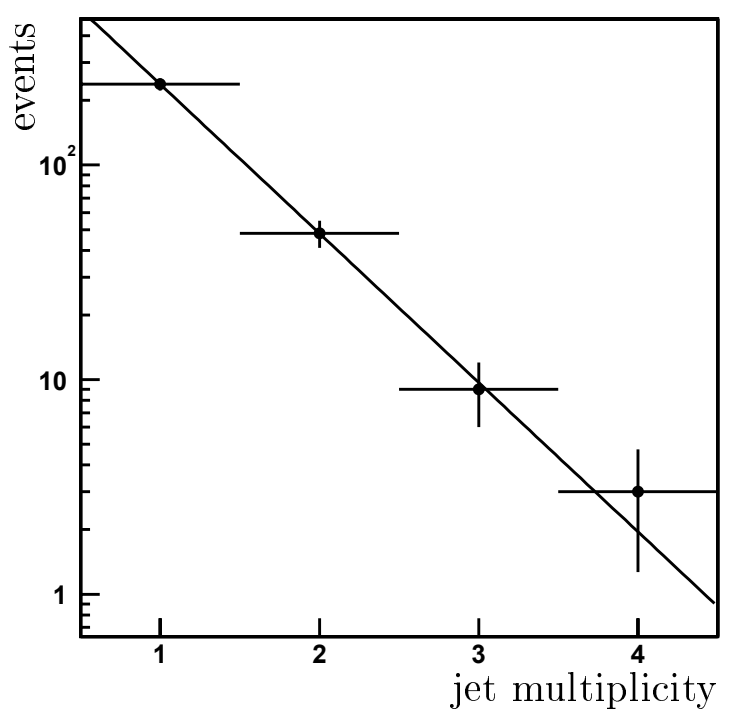

(a)

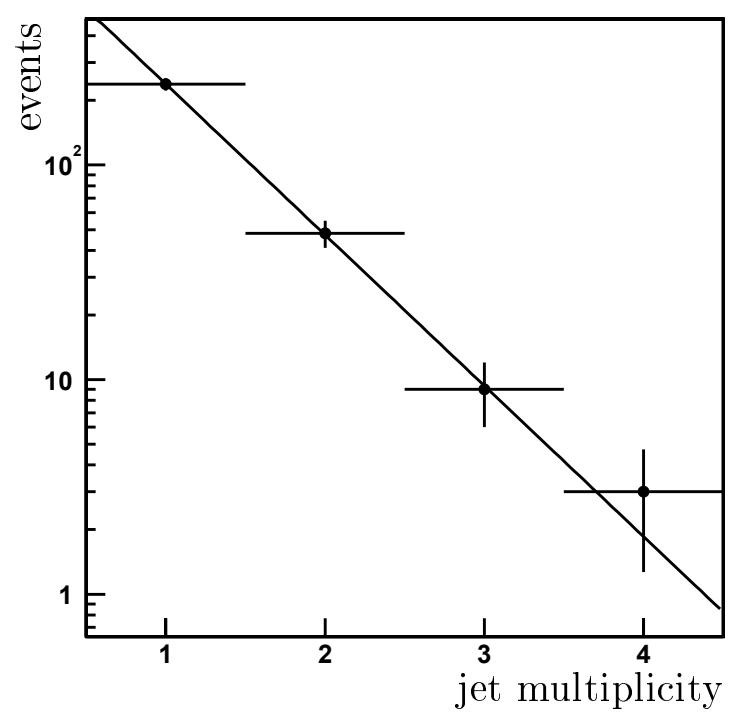

(b)

Figure 8.8: Jet multiplicity for $Z \rightarrow$ ee events. The exponential fit in Figure (a) is to the first two bins, the fit in Figure (b) is to the first three bins.

and use that fit to predict the number of events with a minimum jet multiplicity of four. The fit is shown in Figure 8.8(b). The prediction is 1.9 events, while the actual 
number of events found with four jets (or more) is 3. From this it is clear that within the limited statistics available in the $Z \rightarrow e e$ sample, Berends scaling holds.

In addition, the alternative $W+$ jets background method (Section 8.6), which is independent of Berends scaling, gives a prediction for the $W+$ jets background which is consistent with the result we get from Berends scaling. We therefore conclude that there is no statistically significant systematic uncertainty due to Berends scaling.

\section{NLO correction}

The NLO/LO correction factor in the selection efficiency has a large uncertainty, due to low statistics in the MC samples used. The correction factor is $1.08 \pm 0.05$, giving a systematic uncertainty on the selection efficiency of $5 \%$.

\subsection{Cross section}

In order to estimate the cross section a maximum likelihood method is used. To account for the small number of observed events, Poisson statistics are used. The estimate for the cross section $\sigma$ is given by

$$
\sigma=\frac{N_{o b s}-N_{b k g}}{B r \times \mathcal{L} \times \varepsilon}
$$

where $N_{o b s}, N_{b k g}, B r, \mathcal{L}$ and $\varepsilon$ are respectively the number of observed events, the number of background events, the branching fraction, the integrated luminosity and the overall signal efficiency.

If the cross section is $\sigma$, the expected number of events is

$$
\tilde{N}=\sigma \times B r \times \mathcal{L} \times \varepsilon+N_{b k g} .
$$

The likelihood to observe $N_{\text {obs }}$ events when $\tilde{N}$ events are expected is

$$
\mathcal{L}\left(\sigma,\left\{N_{o b s}, N_{b k g}, B r, \mathcal{L}, \varepsilon\right\}\right)=\frac{\tilde{N}^{N_{o b s}}}{N_{o b s} !} e^{-\tilde{N}}
$$

The cross section is then estimated by minimization of

$$
-2 \log \mathcal{L}\left(\sigma,\left\{N_{\text {obs }}, N_{b k g}, B r, \mathcal{L}, \varepsilon\right\}\right) .
$$

The likelihood function is shown in Figure 8.9.

This function is also used to derive the statistical uncertainty on the cross section measurement. If a probability distribution is Gaussian, its $1 \sigma$ width is measured at $\frac{1}{\sqrt{e}}$ from the peak value. This means that in the log likelihood formulation, the points 


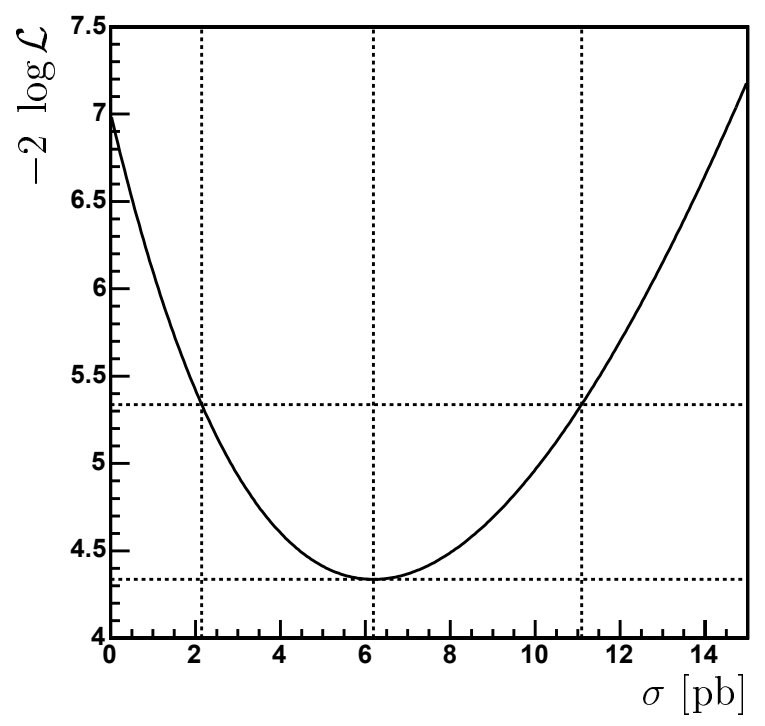

Figure 8.9: Likelihood function.

where -2 times the log of the likelihood function is one higher than the minimum give the statistical uncertainty.

The last step is to estimate the systematic uncertainty on the cross section. We do this by deriving the systematic uncertainty due to each effect (listed in Table 8.9) separately. The affected parameters $\left(\varepsilon, N_{W}, N_{Q C D}, \mathcal{L}\right.$ and/or $\left.B r\right)$ are varied up and down by one $\sigma$ (where correlations between the different parameters are kept) to get the uncertainty on the cross section due to that effect. As we take the different systematic effects to be uncorrelated, we get the final systematic uncertainty on the cross section by adding all the effects in quadrature.

Putting all this together, we get a final $t \bar{t}$ cross section estimate from the $e+$ jets channel of

$$
\sigma_{t \bar{t}}=6.2{ }_{-4.0}^{+4.9}(\text { stat }){ }_{-2.7}^{+2.8}(\text { syst }) \quad \mathrm{pb} .
$$

\subsection{Combined cross section}

For the Lepton Photon 2003 conference, an earlier version of the cross section measurement presented in this thesis was combined with Run II measurements in several other channels. The other channels are the dilepton channels, the $\mu+$ jets channel, and the lepton+jets soft muon tag channels. In the dilepton channels, both top quarks in the event decay to leptons. The dilepton channels used where $e e, \mu \mu$ and $e \mu$. The $\mu+$ jets channel is basically the same as the $e+$ jets channel described in this thesis, except that the $W$ from the top decays to a muon instead of an electron (the other top still decays hadronically). The soft muon tag channels also look at events where one 


\begin{tabular}{c|rccc}
\hline \hline channel & \multicolumn{4}{|c}{$\sigma[\mathrm{pb}]$} \\
\hline \hline$e e$ & 15.8 & ${ }_{-12.3}^{+19.8}$ (stat) & ${ }_{-6.1}^{+6.2}$ (syst) & \pm 1.6 (lumi) \\
\hline$\mu \mu$ & \multicolumn{4}{|c}{$<7.7$ (stat) } \\
\hline$e \mu$ & 9.7 & ${ }_{-5.7}^{+8.4}$ (stat) & ${ }_{-1.9}^{+2.1}$ (syst) & \pm 1.0 (lumi) \\
\hline \hline$e+$ jets & 6.8 & ${ }_{-4.1}^{+5.0}$ (stat) & ${ }_{-2.5}^{+2.6}$ (syst) & \pm 0.7 (lumi) \\
\hline$\mu+$ jets & 2.3 & ${ }_{-3.5}^{+4.2}$ (stat) & ${ }_{-2.6}^{+2.5}$ (syst) & \pm 0.2 (lumi) \\
\hline \hline$e+$ jets $/ \mu$ & 14.2 & ${ }_{-5.6}^{+7.3}$ (stat) & ${ }_{-2.8}^{+2.9}$ (syst) & \pm 1.4 (lumi) \\
\hline$\mu+$ jets $/ \mu$ & 9.5 & ${ }_{-4.1}^{+5.2}$ (stat) & ${ }_{-2.1}^{+2.1}$ (syst) & \pm 0.9 (lumi) \\
\hline \hline
\end{tabular}

Table 8.10: The measured $t \bar{t}$ cross sections for each individual channel.

of the $W$ 's from the top decays leptonically (either to $e$ or $\mu$ ), but in addition require that a muon is found in one of the jets. This indicates that the jet originated from a heavy quark (bottom or charm). Due to the presence of two bottom quarks (from the top decays) and possible one charm quark (from the hadronic W decay) in $t \bar{t}$ events, and minimal heavy quark content in $W+$ jets and QCD events, this cut considerable improves the signal over background ratio. These soft muon tag channels are denoted $e+\mathrm{jets} / \mu$ and $\mu+\mathrm{jets} / \mu$. The $t \bar{t}$ cross sections measured in each of the channels are given in Table 8.10 .

Both the central value and the uncertainties shown for the $e+$ jets channel in the table are somewhat different from the results found in the previous section. This is because several improvements have been made to the analysis as presented in this thesis, leading to a more reliable analysis. The most important are:

- The jets in the MC are now smeared so that they have the same energy resolution as measured in data.

- All systematic uncertainties were recalculated, and additional sources of systematic uncertainty were studied.

- The signal efficiency is corrected for NLO effects.

The measurements in the individual channels can be combined into a single measurement of the cross section. The method used, including the treatment of correlated systematics, is explained in [124]. The combination gives a cross section measurement of

$$
\sigma_{t \bar{t}}=8.1{ }_{-2.0}^{+2.2} \text { (stat) }{ }_{-1.4}^{+1.6} \text { (syst) } \pm 0.8 \text { (lumi) } \mathrm{pb} .
$$

The probability for the observations to be compatible with background processes only is one in one hundred thousand, corresponding to 4.4 standard deviations. This is very close to a rediscovery of the top quark in Run II. 
The combined result, together with D $\varnothing$ Run I measurement of the $t \bar{t}$ cross section and the theoretical prediction, is shown in Figure 8.10 as a function of center of mass energy.

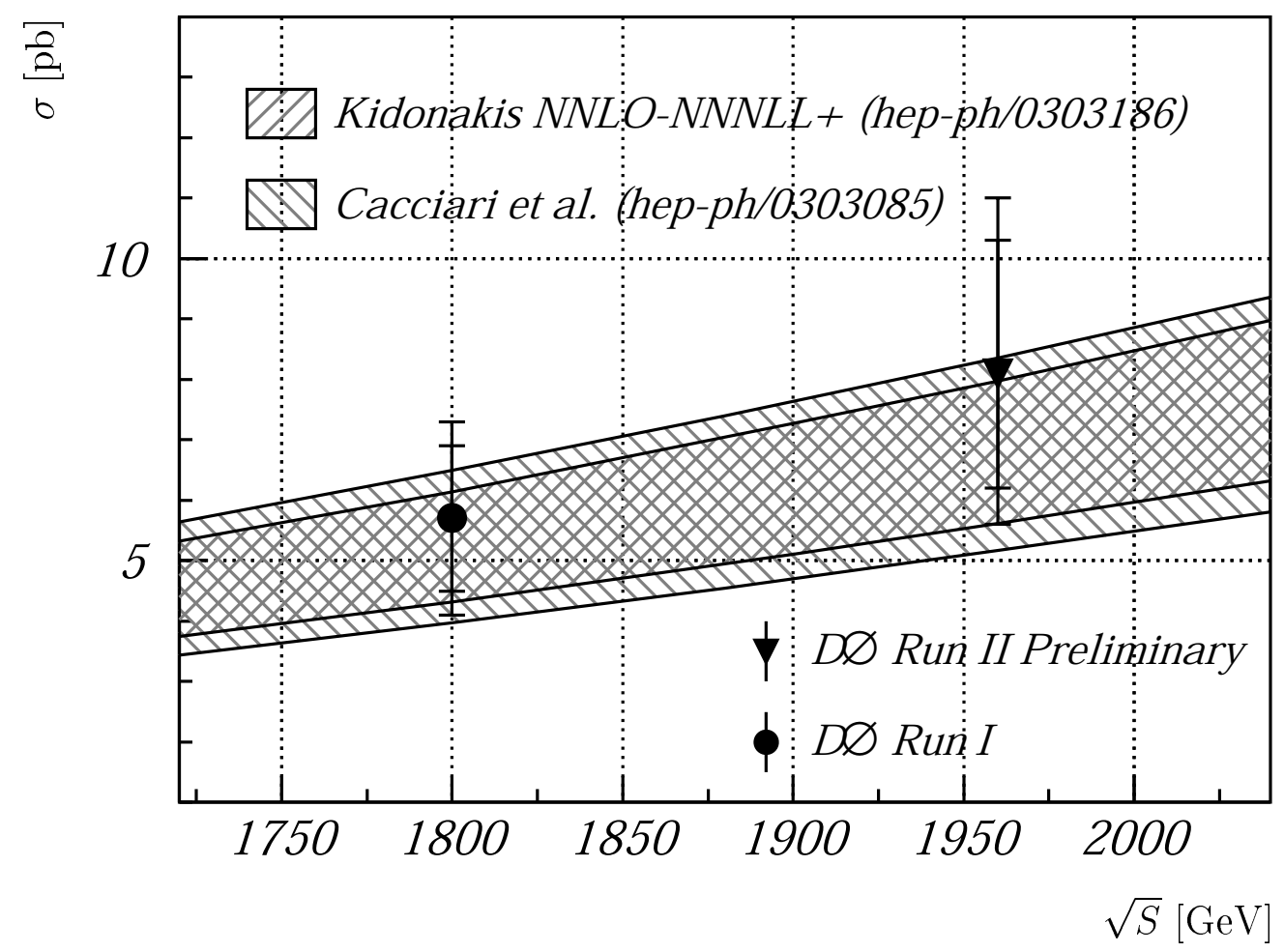

Figure 8.10: DØ measurements of the $t \bar{t}$ cross section at Run I and Run II together with the theoretical prediction. The uncertainty on the theoretical prediction is dominated by the uncertainty on the measurement of the top mass.

\subsection{Conclusion and outlook}

A measurement of the top pair cross section in the $e+$ jets channel at the new Tevatron center of mass energy of $1.96 \mathrm{TeV}$ was presented. Combining the $e+$ jets channel with measurements in other $t \bar{t}$ decay channels, the process was observed at the $4.4 \sigma$ level. The measured cross section is consistent with Standard Model predictions.

In Chapter 1 it was explained why studying the properties of the top quark is important. It should be noted that any measurement on top quarks has to start with the selection of a sample that is enriched with top quark events, and the estimation of backgrounds therein. The experimental methods described in this thesis can thus be important building blocks for other top quark analyses. 
This includes single-top studies, as leptonic single-top events have similar event signatures (lepton, $E_{T}$, two or three jets) and backgrounds ( $W+$ jets, QCD). The observation of single-top production at the $5 \sigma$ level will require about $400 \mathrm{pb}^{-1}$ of integrated luminosity. This amount of data is expected to be available to the CDF and DØ experiments by the end of 2004, and the discovery of the single-top production process should soon follow. With even more integrated luminosity available (the current plan is for the Tevatron to deliver $4 \mathrm{fb}^{-1}$ to each of the experiments before 2009), precision studies of all the top quark properties will get underway. 


\section{Appendix A}

\section{Leading order helicity amplitudes}

In this appendix we have collected the partial helicity amplitudes calculated in Chapter 2 .

\section{A.1 Helicity amplitudes for W-gluon fusion}

We give here the helicity amplitudes for the configurations $\left(p_{3}^{-}, p_{4}^{+}, p_{5}^{+}\right),\left(p_{3}^{-}, p_{4}^{+}, p_{5}^{-}\right)$, $\left(p_{3}^{+}, p_{4}^{-}, p_{5}^{+}\right)$and $\left(p_{3}^{+}, p_{4}^{-}, p_{5}^{-}\right)$.

$$
\begin{aligned}
& A_{W g}^{(1,1)}=\frac{4 \sqrt{2} i B_{W g}^{(1,1)}}{\left(s_{12}-m_{W}^{2}\right)\left(s_{67}-m_{W}^{2}\right)}, \\
& B_{W g}^{(1,1)}\left(p_{3}^{-}, p_{4}^{+}, p_{5}^{+}\right)= \frac{1}{s_{123}-m^{2}} \frac{\langle 31\rangle}{\langle 35\rangle}\left(\frac{\langle 3-|4+5| 7-\rangle\langle 6-|1+3| 2-\rangle}{\langle 54\rangle}\right. \\
&\left.+\frac{[47]}{s_{467}-m^{2}}\left([21]\langle 13\rangle\langle 6-|4+7| 5-\rangle-m^{2}[25]\langle 36\rangle\right)\right), \\
& B_{W g}^{(1,1)}\left(p_{3}^{-}, p_{4}^{+}, p_{5}^{-}\right)= \frac{1}{s_{467}-m^{2}} \frac{[74]}{[54]}\left(\frac{\langle 1-|3+5| 4-\rangle\langle 6-|4+7| 2-\rangle}{[35]}\right. \\
&\left.+\frac{\langle 13\rangle}{s_{123}-m^{2}}\left(\langle 67\rangle[74]\langle 5-|1+3| 2-\rangle-m^{2}\langle 56\rangle[24]\right)\right), \\
& B_{W g}^{(1,1)}\left(p_{3}^{+}, p_{4}^{-}, p_{5}^{+}\right)= 0, \\
& B_{W g}^{(1,1)}\left(p_{3}^{+}, p_{4}^{-}, p_{5}^{-}\right)= 0,
\end{aligned}
$$

$$
A_{W g}^{(1,2)}=\frac{4 \sqrt{2} i B_{W g}^{(1,2)}}{\left(s_{12}-m_{W}^{2}\right) s_{345} s_{127}}
$$




$$
\begin{aligned}
& B_{W g}^{(1,2)}\left(p_{3}^{-}, p_{4}^{+}, p_{5}^{+}\right)=\frac{\langle 36\rangle}{\langle 54\rangle\langle 35\rangle}[27]\langle 1-|(2+7)(4+5)| 3+\rangle, \\
& B_{W g}^{(1,2)}\left(p_{3}^{-}, p_{4}^{+}, p_{5}^{-}\right)=\frac{[27]}{[54][53]}\langle 6-|3+5| 4-\rangle\langle 1-|2+7| 4-\rangle, \\
& B_{W g}^{(1,2)}\left(p_{3}^{+}, p_{4}^{-}, p_{5}^{+}\right)=\frac{\langle 64\rangle}{\langle 45\rangle\langle 35\rangle}[27]\langle 4-|(3+5)(2+7)| 1+\rangle, \\
& B_{W g}^{(1,2)}\left(p_{3}^{+}, p_{4}^{-}, p_{5}^{-}\right)=\frac{[27]}{[45][53]}\langle 1-|2+7| 3-\rangle\langle 6-|4+5| 3-\rangle, \\
& A_{W g}^{(1,3)}=\frac{4 \sqrt{2} i B_{W g}^{(1,3)}}{\left(s_{12}-m_{W}^{2}\right) s_{345} s_{126}}, \\
& B_{W g}^{(1,3)}\left(p_{3}^{-}, p_{4}^{+}, p_{5}^{+}\right)=\frac{\langle 61\rangle}{\langle 45\rangle\langle 35\rangle}\langle 3-|1+6| 2-\rangle\langle 3-|4+5| 7-\rangle, \\
& B_{W g}^{(1,3)}\left(p_{3}^{-}, p_{4}^{+}, p_{5}^{-}\right)=\frac{[47]}{[54][53]}\langle 61\rangle\langle 4+|(3+5)(1+6)| 2-\rangle, \\
& B_{W g}^{(1,3)}\left(p_{3}^{+}, p_{4}^{-}, p_{5}^{+}\right)=\frac{\langle 61\rangle}{\langle 45\rangle\langle 53\rangle}\langle 4-|1+6| 2-\rangle\langle 4-|3+5| 7-\rangle, \\
& B_{W g}^{(1,3)}\left(p_{3}^{+}, p_{4}^{-}, p_{5}^{-}\right)=\frac{[37]}{[54][53]}\langle 61\rangle\langle 2+|(1+6)(4+5)| 3-\rangle, \\
& A_{W g}^{(1,4)}=\frac{4 \sqrt{2} i B_{W g}^{(1,4)}}{\left(s_{12}-m_{W}^{2}\right)\left(s_{67}-m_{W}^{2}\right) s_{345}}, \\
& B_{W g}^{(1,4)}\left(p_{3}^{-}, p_{4}^{+}, p_{5}^{+}\right)=\frac{1}{\langle 54\rangle\langle 35\rangle}(\langle 31\rangle\langle 6-|1+2| 7-\rangle\langle 3-|4+5| 2-\rangle \\
& -\langle 36\rangle\langle 1-|6+7| 2-\rangle\langle 3-|4+5| 7-\rangle+\langle 16\rangle[72]\langle 3-|(6+7)(4+5)| 3+\rangle), \\
& B_{W g}^{(1,4)}\left(p_{3}^{-}, p_{4}^{+}, p_{5}^{-}\right)=\frac{1}{[53][54]}([24]\langle 6-|1+2| 7-\rangle\langle 1-|3+5| 4-\rangle \\
& -[74]\langle 1-|6+7| 2-\rangle\langle 6-|3+5| 4-\rangle-[72]\langle 16\rangle\langle 4+|(6+7)(3+5)| 4-\rangle), \\
& B_{W g}^{(1,4)}\left(p_{3}^{+}, p_{4}^{-}, p_{5}^{+}\right)=\frac{1}{\langle 45\rangle\langle 53\rangle}(\langle 14\rangle\langle 6-|1+2| 7-\rangle\langle 4-|3+5| 2-\rangle \\
& -\langle 64\rangle\langle 1-|6+7| 2-\rangle\langle 4-|3+5| 7-\rangle+\langle 16\rangle[72]\langle 4-|(3+5)(6+7)| 4+\rangle), \\
& B_{W g}^{(1,4)}\left(p_{3}^{+}, p_{4}^{-}, p_{5}^{-}\right)=\frac{1}{[54][53]}([32]\langle 6-|1+2| 7-\rangle\langle 1-|4+5| 3-\rangle \\
& -[37]\langle 1-|6+7| 2-\rangle\langle 6-|4+5| 3-\rangle+\langle 16\rangle[72]\langle 3+|(6+7)(4+5)| 3-\rangle),
\end{aligned}
$$




$$
\begin{aligned}
& A_{W g}^{(1,5)}=\frac{4 \sqrt{2} i B_{W g}^{(1,5)}}{\left(s_{67}-m_{W}^{2}\right) s_{345} s_{167}} \\
& B_{W g}^{(1,5)}\left(p_{3}^{-}, p_{4}^{+}, p_{5}^{+}\right)=\frac{\langle 16\rangle}{\langle 35\rangle\langle 45\rangle}\langle 3-|1+6| 7-\rangle\langle 3-|4+5| 2-\rangle, \\
& B_{W g}^{(1,5)}\left(p_{3}^{-}, p_{4}^{+}, p_{5}^{-}\right)=\frac{[24]}{[45][35]}\langle 16\rangle\langle 7+|(1+6)(3+5)| 4-\rangle, \\
& B_{W g}^{(1,5)}\left(p_{3}^{+}, p_{4}^{-}, p_{5}^{+}\right)=-\frac{\langle 16\rangle}{\langle 35\rangle\langle 45\rangle}\langle 4-|1+6| 7-\rangle\langle 4-|3+5| 2-\rangle, \\
& B_{W g}^{(1,5)}\left(p_{3}^{+}, p_{4}^{-}, p_{5}^{-}\right)=-\frac{[23]}{[35][45]}\langle 16\rangle\langle 7+|(1+6)(4+5)| 3-\rangle, \\
& A_{W g}^{(1,6)}=\frac{4 \sqrt{2} i B_{W g}^{(1,6)}}{\left(s_{67}-m_{W}^{2}\right) s_{345} s_{267}} \\
& B_{W g}^{(1,6)}\left(p_{3}^{-}, p_{4}^{+}, p_{5}^{+}\right)=\frac{\langle 13\rangle}{\langle 35\rangle\langle 45\rangle}[27]\langle 3-|(4+5)(2+7)| 6+\rangle, \\
& B_{W g}^{(1,6)}\left(p_{3}^{-}, p_{4}^{+}, p_{5}^{-}\right)=-\frac{[27]}{[35][45]}\langle 1-|3+5| 4-\rangle\langle 6-|2+7| 4-\rangle, \\
& B_{W g}^{(1,6)}\left(p_{3}^{+}, p_{4}^{-}, p_{5}^{+}\right)=-\frac{\langle 14\rangle}{\langle 35\rangle\langle 45\rangle}[27]\langle 4-|(3+5)(2+7)| 6+\rangle, \\
& B_{W g}^{(1,6)}\left(p_{3}^{+}, p_{4}^{-}, p_{5}^{-}\right)=\frac{[27]}{[35][45]}\langle 1-|4+5| 3-\rangle\langle 6-|2+7| 3-\rangle, \\
& A_{W g}^{(2,1)}=\frac{4 \sqrt{2} i B_{W g}^{(2,1)}}{\left(s_{12}-m_{W}^{2}\right)\left(s_{567}-m_{W}^{2}\right)\left(s_{123}-m^{2}\right)}, \\
& B_{W g}^{(2,1)}\left(p_{3}^{-}, p_{4}^{+}, p_{5}^{+}\right)=\frac{\langle 31\rangle}{\langle 65\rangle\langle 75\rangle}\langle 6-|1+3| 2-\rangle\langle 6-|5+7| 4-\rangle, \\
& B_{W g}^{(2,1)}\left(p_{3}^{-}, p_{4}^{+}, p_{5}^{-}\right)=\frac{[47]}{[57][56]}\langle 31\rangle\langle 2+|(1+3)(5+6)| 7-\rangle, \\
& B_{W g}^{(2,1)}\left(p_{3}^{+}, p_{4}^{-}, p_{5}^{+}\right)=0, \\
& B_{W g}^{(2,1)}\left(p_{3}^{+}, p_{4}^{-}, p_{5}^{-}\right)=0, \\
& A_{W g}^{(2,2)}=\frac{4 \sqrt{2} i B_{W g}^{(2,2)}}{\left(s_{12}-m_{W}^{2}\right) s_{34}}
\end{aligned}
$$




$$
\begin{aligned}
& B_{W g}^{(2,2)}\left(p_{3}^{-}, p_{4}^{+}, p_{5}^{+}\right)= \\
& \frac{1}{s_{346}} \frac{\langle 63\rangle}{\langle 65\rangle}\left(\frac{\langle 1-|3+6| 4-\rangle\langle 6-|5+7| 2-\rangle}{\langle 57\rangle}-\frac{[43]\langle 36\rangle}{s_{127}}[27]\langle 1-|2+7| 5-\rangle\right), \\
& B_{W g}^{(2,2)}\left(p_{3}^{-}, p_{4}^{+}, p_{5}^{-}\right)= \\
& -\frac{1}{s_{127}} \frac{[27]}{[57]}\left(\frac{\langle 3-|5+6| 7-\rangle\langle 1-|2+7| 4-\rangle}{[56]}+\frac{\langle 63\rangle\langle 12\rangle[27]\langle 5-|3+6| 4-\rangle}{s_{346}}\right), \\
& B_{W g}^{(2,2)}\left(p_{3}^{+}, p_{4}^{-}, p_{5}^{+}\right)= \\
& \frac{1}{s_{346}} \frac{\langle 64\rangle}{\langle 65\rangle}\left(\frac{\langle 1-|4+6| 3-\rangle\langle 6-|5+7| 2-\rangle}{\langle 57\rangle}-\frac{[34]\langle 46\rangle}{s_{127}}[27]\langle 1-|2+7| 5-\rangle\right), \\
& B_{W g}^{(2,2)}\left(p_{3}^{+}, p_{4}^{-}, p_{5}^{-}\right)= \\
& -\frac{1}{s_{127}} \frac{[27]}{[57]}\left(\frac{\langle 4-|5+6| 7-\rangle\langle 1-|2+7| 3-\rangle}{[56]}+\frac{\langle 64\rangle\langle 12\rangle[27]\langle 5-|4+6| 3-\rangle}{s_{346}}\right), \\
& A_{W g}^{(2,3)}=\frac{4 \sqrt{2} i B_{W g}^{(2,3)}}{\left(s_{12}-m_{W}^{2}\right) s_{34}}, \\
& B_{W g}^{(2,3)}\left(p_{3}^{-}, p_{4}^{+}, p_{5}^{+}\right)= \\
& \frac{1}{s_{126}} \frac{\langle 61\rangle}{\langle 65\rangle}\left(\frac{\langle 3-|1+6| 2-\rangle\langle 6-|5+7| 4-\rangle}{\langle 57\rangle}-\frac{[21]\langle 16\rangle[47]\langle 3-|4+7| 5-\rangle}{s_{347}}\right), \\
& B_{W g}^{(2,3)}\left(p_{3}^{-}, p_{4}^{+}, p_{5}^{-}\right)= \\
& -\frac{1}{s_{347}} \frac{[47]}{[57]}\left(\frac{\langle 1-|5+6| 7-\rangle\langle 3-|4+7| 2-\rangle}{[56]}-\frac{\langle 61\rangle[74]\langle 34\rangle\langle 5-|1+6| 2-\rangle}{s_{126}}\right), \\
& B_{W g}^{(2,3)}\left(p_{3}^{+}, p_{4}^{-}, p_{5}^{+}\right)= \\
& \frac{1}{s_{126}} \frac{\langle 61\rangle}{\langle 65\rangle}\left(\frac{\langle 4-|1+6| 2-\rangle\langle 6-|5+7| 3-\rangle}{\langle 57\rangle}-\frac{[21]\langle 16\rangle[37]\langle 4-|3+7| 5-\rangle}{s_{347}}\right), \\
& B_{W g}^{(2,3)}\left(p_{3}^{+}, p_{4}^{-}, p_{5}^{-}\right)= \\
& -\frac{1}{s_{347}} \frac{[37]}{[57]}\left(\frac{\langle 1-|5+6| 7-\rangle\langle 4-|3+7| 2-\rangle}{[56]}-\frac{\langle 61\rangle[73]\langle 43\rangle\langle 5-|1+6| 2-\rangle}{s_{126}}\right), \\
& A_{W g}^{(2,4)}=\frac{4 \sqrt{2} i B_{W g}^{(2,4)}}{\left(s_{12}-m_{W}^{2}\right)\left(s_{567}-m_{W}^{2}\right) s_{34}}, \\
& B_{W g}^{(2,4)}\left(p_{3}^{-}, p_{4}^{+}, p_{5}^{+}\right)=-\frac{1}{\langle 75\rangle\langle 65\rangle}(\langle 63\rangle\langle 1-|3+4| 2-\rangle\langle 6-|5+7| 4-\rangle
\end{aligned}
$$




$$
\begin{aligned}
& -\langle 61\rangle\langle 3-|1+2| 4-\rangle\langle 6-|5+7| 2-\rangle+\langle 13\rangle[42]\langle 6-|(1+2)(5+7)| 6+\rangle), \\
& B_{W g}^{(2,4)}\left(p_{3}^{-}, p_{4}^{+}, p_{5}^{-}\right)=-\frac{1}{[57][65]}([47]\langle 1-|3+4| 2-\rangle\langle 3-|5+6| 7-\rangle \\
& -[27]\langle 3-|1+2| 4-\rangle\langle 1-|5+6| 7-\rangle+[42]\langle 13\rangle\langle 7+|(5+6)(1+2)| 7-\rangle), \\
& B_{W g}^{(2,4)}\left(p_{3}^{+}, p_{4}^{-}, p_{5}^{+}\right)=-\frac{1}{\langle 75\rangle\langle 65\rangle}(\langle 64\rangle\langle 1-|3+4| 2-\rangle\langle 6-|5+7| 3-\rangle \\
& -\langle 61\rangle\langle 4-|1+2| 3-\rangle\langle 6-|5+7| 2-\rangle+\langle 14\rangle[32]\langle 6-|(1+2)(5+7)| 6+\rangle), \\
& B_{W g}^{(2,4)}\left(p_{3}^{+}, p_{4}^{-}, p_{5}^{-}\right)=-\frac{1}{[57][65]}([37]\langle 1-|3+4| 2-\rangle\langle 4-|5+6| 7-\rangle \\
& -[27]\langle 4-|1+2| 3-\rangle\langle 1-|5+6| 7-\rangle+[32]\langle 14\rangle\langle 7+|(5+6)(1+2)| 7-\rangle), \\
& A_{W g}^{(2,5)}=\frac{4 \sqrt{2} i B_{W g}^{(2,5)}}{\left(s_{567}-m_{W}^{2}\right) s_{34} s_{234}} \\
& B_{W g}^{(2,5)}\left(p_{3}^{-}, p_{4}^{+}, p_{5}^{+}\right)=\frac{\langle 16\rangle}{\langle 56\rangle\langle 57\rangle}[24]\langle 6-|(5+7)(2+4)| 3+\rangle, \\
& B_{W g}^{(2,5)}\left(p_{3}^{-}, p_{4}^{+}, p_{5}^{-}\right)=-\frac{[24]}{[56][57]}\langle 1-|5+6| 7-\rangle\langle 3-|2+4| 7-\rangle, \\
& B_{W g}^{(2,5)}\left(p_{3}^{+}, p_{4}^{-}, p_{5}^{+}\right)=\frac{\langle 16\rangle}{\langle 56\rangle\langle 57\rangle}[23]\langle 6-|(5+7)(2+3)| 4+\rangle, \\
& B_{W g}^{(2,5)}\left(p_{3}^{+}, p_{4}^{-}, p_{5}^{-}\right)=-\frac{[23]}{[56][57]}\langle 1-|5+6| 7-\rangle\langle 4-|2+3| 7-\rangle, \\
& A_{W g}^{(2,6)}=\frac{4 \sqrt{2} i B_{W g}^{(2,6)}}{\left(s_{567}-m_{W}^{2}\right) s_{34} s_{134}} \\
& B_{W g}^{(2,6)}\left(p_{3}^{-}, p_{4}^{+}, p_{5}^{+}\right)=\frac{\langle 13\rangle}{\langle 56\rangle\langle 57\rangle}\langle 6-|1+3| 4-\rangle\langle 6-|5+7| 2-\rangle, \\
& B_{W g}^{(2,6)}\left(p_{3}^{-}, p_{4}^{+}, p_{5}^{-}\right)=\frac{[27]}{[56][57]}\langle 13\rangle\langle 4+|(1+3)(5+6)| 7-\rangle, \\
& B_{W g}^{(2,6)}\left(p_{3}^{+}, p_{4}^{-}, p_{5}^{+}\right)=\frac{\langle 14\rangle}{\langle 56\rangle\langle 57\rangle}\langle 6-|1+4| 3-\rangle\langle 6-|5+7| 2-\rangle, \\
& B_{W g}^{(2,6)}\left(p_{3}^{+}, p_{4}^{-}, p_{5}^{-}\right)=\frac{[27]}{[56][57]}\langle 14\rangle\langle 3+|(1+4)(5+6)| 7-\rangle .
\end{aligned}
$$




\section{A.2 Helicity amplitudes for flavor excitation and the $s$-channel}

We give here the helicity amplitudes for the configurations $\left(p_{3}^{-}, p_{4}^{+}\right)$, and $\left(p_{3}^{+}, p_{4}^{-}\right)$.

$$
\begin{aligned}
& A_{W b}^{(1,1)}=\frac{-4 i B_{W b}^{(1,1)}}{\left(s_{12}-m_{W}^{2}\right)\left(s_{67}-m_{W}^{2}\right)\left(s_{123}-m^{2}\right)}, \\
& B_{W b}^{(1,1)}\left(p_{3}^{-}, p_{4}^{+}\right)=\langle 31\rangle[74]\langle 6-|1+3| 2-\rangle, \\
& B_{W b}^{(1,1)}\left(p_{3}^{+}, p_{4}^{-}\right)=0, \\
& A_{W b}^{(1,2)}=\frac{-4 i B_{W b}^{(1,2)}}{\left(s_{12}-m_{W}^{2}\right) s_{34} s_{346}}, \\
& B_{W b}^{(1,2)}\left(p_{3}^{-}, p_{4}^{+}\right)=\langle 63\rangle[27]\langle 1-|3+6| 4-\rangle, \\
& B_{W b}^{(1,2)}\left(p_{3}^{+}, p_{4}^{-}\right)=\langle 64\rangle[27]\langle 1-|4+6| 3-\rangle, \\
& A_{W b}^{(1,3)}=\frac{-4 i B_{W b}^{(1,3)}}{\left(s_{12}-m_{W}^{2}\right) s_{34} s_{126}} \\
& B_{W b}^{(1,3)}\left(p_{3}^{-}, p_{4}^{+}\right)=\langle 61\rangle[47]\langle 3-|1+6| 2-\rangle, \\
& B_{W b}^{(1,3)}\left(p_{3}^{+}, p_{4}^{-}\right)=\langle 61\rangle[37]\langle 4-|1+6| 2-\rangle \text {, } \\
& A_{W b}^{(1,4)}=\frac{-4 i B_{W b}^{(1,4)}}{\left(s_{12}-m_{W}^{2}\right)\left(s_{67}-m_{W}^{2}\right) s_{34}} \\
& B_{W b}^{(1,4)}\left(p_{3}^{-}, p_{4}^{+}\right)=-\langle 63\rangle[47]\langle 1-|6+7| 2-\rangle-\langle 13\rangle[42]\langle 6-|3+4| 7-\rangle \\
& -\langle 61\rangle[27]\langle 3-|1+2| 4-\rangle \text {, } \\
& B_{W b}^{(1,4)}\left(p_{3}^{+}, p_{4}^{-}\right)=-\langle 64\rangle[37]\langle 1-|6+7| 2-\rangle-\langle 14\rangle[32]\langle 6-|3+4| 7-\rangle \\
& -\langle 61\rangle[27]\langle 4-|1+2| 3-\rangle, \\
& A_{W b}^{(1,5)}=\frac{-4 i B_{W b}^{(1,5)}}{\left(s_{67}-m_{W}^{2}\right) s_{34} s_{167}}
\end{aligned}
$$




$$
\begin{aligned}
B_{W b}^{(1,5)}\left(p_{3}^{-}, p_{4}^{+}\right) & =\langle 16\rangle[42]\langle 3-|1+6| 7-\rangle, \\
B_{W b}^{(1,5)}\left(p_{3}^{+}, p_{4}^{-}\right) & =\langle 16\rangle[32]\langle 4-|1+6| 7-\rangle, \\
A_{W b}^{(1,6)} & =\frac{-4 i B_{W b}^{(1,6)}}{\left(s_{67}-m_{W}^{2}\right) s_{34} s_{134}}, \\
B_{W b}^{(1,6)}\left(p_{3}^{-}, p_{4}^{+}\right) & =\langle 13\rangle[72]\langle 6-|1+3| 4-\rangle, \\
B_{W b}^{(1,6)}\left(p_{3}^{+}, p_{4}^{-}\right) & =\langle 14\rangle[72]\langle 6-|1+4| 3-\rangle .
\end{aligned}
$$




\section{Appendix B}

\section{Scalar integrals}

Expressions for the scalar integrals encountered in Chapter 4 are derived in this appendix. We calculate the integrals in $D=4-2 \varepsilon$ dimensions and use the notation

$$
\Delta=\frac{1}{\varepsilon}-\gamma+\ln 4 \pi \text {. }
$$

All integrals are calculated in the Euclidean region (invariants $p^{2}<0$ and masses $\left.m^{2}>0\right)$. These expressions can then be continued analytically to the regions of interest, using the substitution $-s \rightarrow-s-i \varepsilon$ (where $\varepsilon$ denotes a small parameter, not to be confused with the one appearing in dimensional regularization) and the formula

$$
\ln (-s-i \varepsilon)=\ln (|s|)-i \pi \Theta(s) .
$$

Massive integrals: The tadpole is given for $m^{2}>0$ by

$$
\begin{aligned}
A_{0}\left(m^{2}\right) & =(4 \pi)^{2} \mu^{4-D} \int \frac{d^{D} k}{(2 \pi)^{D} i} \frac{1}{k^{2}-m^{2}}=-m^{2} \Gamma(-1+\varepsilon)(4 \pi)^{\varepsilon}\left(\frac{m^{2}}{\mu^{2}}\right)^{-\varepsilon} \\
& =m^{2}\left(\Delta+1-\ln \frac{m^{2}}{\mu^{2}}\right)+O(\varepsilon) .
\end{aligned}
$$

The bubble with one internal mass is given for $-p^{2}>0, m^{2}>0$ by

$$
\begin{aligned}
B_{0}^{(a)}\left(p^{2}, m^{2}\right) & =(4 \pi)^{2} \mu^{4-D} \int \frac{d^{D} k}{(2 \pi)^{D}} i \frac{1}{k^{2}\left((k-p)^{2}-m^{2}\right)} \\
& =\Delta+2-\ln \frac{m^{2}-p^{2}}{\mu^{2}}+\frac{m^{2}}{-p^{2}} \ln \frac{m^{2}}{m^{2}-p^{2}}+O(\varepsilon) .
\end{aligned}
$$

On-shell integrals: The bubble with one internal mass is given for $p^{2}=m^{2}>0$ by

$$
\begin{aligned}
B_{0}^{(b)}\left(m^{2}\right) & =(4 \pi)^{2} \mu^{4-D} \int \frac{d^{D} k}{(2 \pi)^{D} i} \frac{1}{k^{2}\left((k-p)^{2}-m^{2}\right)} \\
& =\Delta+2-\ln \frac{m^{2}}{\mu^{2}}+O(\varepsilon) .
\end{aligned}
$$


The triangle with two external masses and one internal mass, between the two external massive lines is given for $p_{1}^{2}=0,\left(p_{1}+p_{2}\right)^{2}=m^{2}>0$ by

$$
\begin{aligned}
C_{0}^{(a)}\left(p_{2}^{2}, m^{2}\right)=(4 \pi)^{2} \mu^{4-D} \int \frac{d^{D} k}{(2 \pi)^{D} i} \frac{1}{k^{2}\left(k-p_{1}\right)^{2}\left(\left(k-p_{1}-p_{2}\right)^{2}-m^{2}\right)} \\
=\quad\left(4 \pi \mu^{2}\right)^{\varepsilon} \frac{\Gamma(1+\varepsilon)}{m^{2}-p_{2}^{2}}\left\{-\frac{1}{2 \varepsilon^{2}}+\frac{1}{\varepsilon}\left(\ln \left(m^{2}-p_{2}^{2}\right)-\frac{1}{2} \ln \left(m^{2}\right)\right)\right. \\
\left.\quad+\frac{1}{4} \ln ^{2}\left(m^{2}\right)-\frac{1}{2} \ln ^{2}\left(m^{2}-p_{2}^{2}\right)+\operatorname{Li}_{2}\left(\frac{-p_{2}^{2}}{m^{2}-p_{2}^{2}}\right)\right\}+O(\varepsilon) \\
=\frac{1}{m^{2}-p_{2}^{2}}\left\{-\frac{1}{2} \Delta^{2}+\Delta\left(-\frac{1}{2} \gamma+\frac{1}{2} \ln 4 \pi+\frac{1}{2} \ln \frac{m^{2}}{\mu^{2}}+\ln \frac{m^{2}-p_{2}^{2}}{m^{2}}\right)-\frac{1}{4}(\gamma-\ln 4 \pi)^{2}\right. \\
\left.\quad+\frac{1}{4} \ln ^{2}\left(\frac{m^{2}}{\mu^{2}}\right)-\frac{1}{2} \ln ^{2}\left(\frac{m^{2}-p_{2}^{2}}{\mu^{2}}\right)+\operatorname{Li}_{2}\left(\frac{-p_{2}^{2}}{m^{2}-p_{2}^{2}}\right)-\frac{\pi^{2}}{24}\right\}+O(\varepsilon) .
\end{aligned}
$$

Integrals with no internal masses: The bubble with $-p^{2}>0$ is given by

$$
\begin{aligned}
B_{0}^{(c)}\left(p^{2}\right) & =(4 \pi)^{2} \mu^{4-D} \int \frac{d^{D} k}{(2 \pi)^{D} i} \frac{1}{k^{2}(k-p)^{2}}=\Gamma(\varepsilon) \frac{\Gamma^{2}(1-\varepsilon)}{\Gamma(2-2 \varepsilon)}(4 \pi)^{\varepsilon}\left(\frac{-p^{2}}{\mu^{2}}\right)^{-\varepsilon} \\
& =\Delta+2-\ln \frac{-p^{2}}{\mu^{2}}+O(\varepsilon) .
\end{aligned}
$$

The triangle with one external mass $p_{1}^{2}=p_{2}^{2}=0,-s=-2 p_{1} p_{2}>0$ is given by

$$
\begin{aligned}
& C_{0}^{(b)}(s)=(4 \pi)^{2} \mu^{4-D} \int \frac{d^{D} k}{(2 \pi)^{D} i} \frac{1}{k^{2}\left(k-p_{1}\right)^{2}\left(k-p_{1}-p_{2}\right)^{2}} \\
& =\frac{1}{s} \frac{1}{\varepsilon^{2}} \frac{\Gamma(1+\varepsilon) \Gamma^{2}(1-\varepsilon)}{\Gamma(1-2 \varepsilon)}(4 \pi)^{\varepsilon}\left(\frac{-s}{\mu^{2}}\right)^{-\varepsilon} \\
& =\frac{1}{s}\left(\Delta^{2}+\Delta\left(\gamma-\ln 4 \pi-\ln \frac{-s}{\mu^{2}}\right)+\frac{1}{2}(\gamma-\ln 4 \pi)^{2}+\frac{1}{2} \ln ^{2}\left(\frac{-s}{\mu^{2}}\right)-\frac{\pi^{2}}{12}\right)+O(\varepsilon) .
\end{aligned}
$$

Six-dimensional integrals: The triangle with an additional power of $k_{-2 \varepsilon}^{2}$ in the numerator is equivalent to an integral in $D=6-2 \varepsilon$ dimensions and yields for the massless and massive case

$$
\begin{aligned}
C_{0}^{(-2 \varepsilon)} & =(4 \pi)^{2} \mu^{4-D} \int \frac{d^{D} k}{(2 \pi)^{D} i} \frac{k_{(-2 \varepsilon)}^{2}}{k^{2}\left(k-p_{1}\right)^{2}\left(\left(k-p_{1}-p_{2}\right)^{2}-m^{2}\right)} \\
& =-\frac{1}{2}+O(\varepsilon) .
\end{aligned}
$$




\section{Appendix C}

\section{Additional backgrounds}

In this appendix we will discuss the $W+$ jets background where the $W$ decays to a tau and we will show that all background contributions coming from $Z+$ jets events can be neglected.

\section{C.1 $\quad W \rightarrow \tau \nu$ background}

The relative size of this background contribution is estimated from a $W+$ jets Monte Carlo sample. If the $W$ is allowed to decay only to an electron, $4.7 \%$ of the events pass all cuts up to (but excluding) the topological cuts. If the $W$ can only decay to a tau, only $0.5 \%$ of the events survive these cuts. This is so much lower, because less than one fifth of the taus decays to an electron, and these electrons have lower energy than in a direct decay.

In the analysis, the $W+$ jets contribution is a combination of the $W$ events where the $W$ decays directly to an electron and events where the $W$ decays first to a tau. The cascade decays make up about $10 \%$ of the events. As the jets in these events come only from initial state QCD interactions, we expect the jet multiplicity spectra to be the same for both contributions. We can therefore treat them together as one $W+$ jets background in the Berends scaling fit. As the efficiency for the topological cuts for the cascade events $(18 \pm 7 \%)$ is consistent with the one found in events where the $W$ decays directly to an electron, it is correct to treat both $W$ backgrounds together for the topological cuts, as we do in the analysis.

\section{C.2 $Z+$ jets backgrounds}

The $Z+$ jets events can mimic the correct event signature in two ways: 
- The $Z$ decays to electrons, and one of the electrons is either missed, or not identified as an electron.

- The $Z$ decays to taus, where one tau decays to an electron (and two neutrinos), and the other tau decays hadronically.

We study both contributions at once by looking at a Z/Drell-Yan +2 jet Monte Carlo sample, where the $Z$ decays to leptons (either $e^{+} e^{-}$or $\tau^{+} \tau^{-}$). In this Monte Carlo sample, there are 958 events where the $Z$ decays to electrons, both electrons are reconstructed in the $\mathrm{CC}$ and in addition two jets are found. In a data sample of the same integrated luminosity as used in the analysis we find 48 events that pass these requirements. This means that the integrated luminosity of the Monte Carlo sample is roughly twenty times bigger than the $92 \mathrm{pb}^{-1}$ used in the analysis.

If we now apply all the analysis cuts to this same Monte Carlo sample, we see that only 1 event survives. This includes the contributions from the $Z$ decaying to taus or electrons. As the integrated luminosity used for the analysis is only $5 \%$ of what is available in the Monte Carlo sample, the background from $Z$ events after all cuts is $0.05 \pm 0.05$ events, and can thus be neglected. 


\section{References}

[1] F. Mandl and G. Shaw, Quantum Field Theory (John Wiley \& Sons, 1984).

[2] M. E. Peskin and D. V. Schroeder, An Introduction to Quantum Field Theory (Perseus books, 1995).

[3] G. Sterman, An Introduction to Quantum Field Theory (Cambridge University Press, 1993).

[4] J. van der Heide, E. Laenen, L. Phaf, and S. Weinzierl, Phys. Rev. D62, 074025 (2000), hep-ph/0003318.

[5] L. Phaf and S. Weinzierl, JHEP 04, 006 (2001), hep-ph/0102207.

[6] B. W. Harris, E. Laenen, L. Phaf, Z. Sullivan, and S. Weinzierl, Phys. Rev. D66, 054024 (2002), hep-ph/0207055.

[7] I. I. Y. Bigi, Y. L. Dokshitzer, V. A. Khoze, J. H. Kuhn, and P. M. Zerwas, Phys. Lett. B181, 157 (1986).

[8] R. Pittau, Phys. Lett. B386, 397 (1996), hep-ph/9603265.

[9] G. Mahlon and S. Parke, Phys. Lett. B347, 394 (1995), hep-ph/9412250.

[10] N. Kauer and D. Zeppenfeld, Phys. Rev. D65, 014021 (2002), hep-ph/0107181.

[11] T. Stelzer and W. F. Long, Comput. Phys. Commun. 81, 357 (1994), hep$\mathrm{ph} / 9401258$.

[12] D. O. Carlson, Ph.D. Thesis, Michigan State University (1995), hep-ph/9508278.

[13] T. Sjostrand et al., Comput. Phys. Commun. 135, 238 (2001), hep-ph/0010017.

[14] G. Corcella et al., JHEP 01, 010 (2001), hep-ph/0011363.

[15] A. P. Heinson, A. S. Belyaev, and E. E. Boos, Phys. Rev. D56, 3114 (1997), hep-ph/9612424. 
[16] T. Stelzer, Z. Sullivan, and S. Willenbrock, Phys. Rev. D58, 094021 (1998), hep-ph/9807340.

[17] D. A. Dicus and S. S. D. Willenbrock, Phys. Rev. D34, 148 (1986).

[18] A. S. Belyaev, E. E. Boos, and L. V. Dudko, Phys. Rev. D59, 075001 (1999), hep-ph/9806332.

[19] F. A. Berends, R. Kleiss, P. De Causmaecker, R. Gastmans, and T. T. Wu, Phys. Lett. B103, 124 (1981).

[20] R. Kleiss and W. J. Stirling, Nucl. Phys. B262, 235 (1985).

[21] Z. Xu, D.-H. Zhang, and L. Chang, Nucl. Phys. B291, 392 (1987).

[22] J. F. Gunion and Z. Kunszt, Phys. Lett. B161, 333 (1985).

[23] R. Gastmans and T. T. Wu, The Ubiquitous photon: Helicity method for QED and QCD (Clarendon Press, Oxford, 1990).

[24] M. L. Mangano and S. J. Parke, Phys. Rept. 200, 301 (1991).

[25] L. J. Dixon, (1996), hep-ph/9601359.

[26] F. A. Berends, P. H. Daverveldt, and R. Kleiss, Nucl. Phys. B253, 441 (1985).

[27] A. Ballestrero and E. Maina, Phys. Lett. B350, 225 (1995), hep-ph/9403244.

[28] S. Dittmaier, Phys. Rev. D59, 016007 (1999), hep-ph/9805445.

[29] M. Bohm, H. Spiesberger, and W. Hollik, Fortsch. Phys. 34, 687 (1986).

[30] A. Pukhov et al., (1999), hep-ph/9908288.

[31] H. L. Lai et al., Phys. Rev. D55, 1280 (1997), hep-ph/9606399.

[32] A. D. Martin and D. Zeppenfeld, Phys. Lett. B345, 558 (1995), hep-ph/9411377.

[33] S. Eidelman and F. Jegerlehner, Z. Phys. C67, 585 (1995), hep-ph/9502298.

[34] M. L. Swartz, Phys. Rev. D53, 5268 (1996), hep-ph/9509248.

[35] R. Alemany, M. Davier, and A. Hocker, Eur. Phys. J. C2, 123 (1998), hep$\mathrm{ph} / 9703220$.

[36] S. Catani, Y. L. Dokshitzer, M. H. Seymour, and B. R. Webber, Nucl. Phys. B406, 187 (1993). 
[37] A. Denner, S. Dittmaier, M. Roth, and D. Wackeroth, Nucl. Phys. B560, 33 (1999), hep-ph/9904472.

[38] C. P. Yuan, Phys. Rev. D41, 42 (1990).

[39] R. K. Ellis and S. Parke, Phys. Rev. D46, 3785 (1992).

[40] G. Mahlon and S. Parke, Phys. Rev. D55, 7249 (1997), hep-ph/9611367.

[41] G. Mahlon and S. Parke, Phys. Lett. B476, 323 (2000), hep-ph/9912458.

[42] G. Mahlon and S. Parke, Phys. Rev. D53, 4886 (1996), hep-ph/9512264.

[43] M. Jezabek, Nucl. Phys. Proc. Suppl. 37B, 197 (1994), hep-ph/9406411.

[44] CDF, Y.-C. Liu, to be published in the proceedings of 13th Topical Conference on Hadron Collider Physics, Mumbai, India, 14-20 Jan 1999.

[45] S. Catani and M. H. Seymour, Nucl. Phys. B485, 291 (1997), hep-ph/9605323.

[46] K. Fabricius, I. Schmitt, G. Kramer, and G. Schierholz, Zeit. Phys. C11, 315 (1981).

[47] W. L. van Neerven and J. A. M. Vermaseren, Nucl. Phys. B238 (1984).

[48] G. Kramer and B. Lampe, Fortschr. Phys. 37, 161 (1989).

[49] R. K. Ellis, D. A. Ross, and A. E. Terrano, Nucl. Phys. B178, 421 (1981).

[50] Z. Kunszt and P. Nason, in "Z Physics at LEP 1", Proceedings of the 1989 LEP Physics Workshop, Geneva, ed. by G. Altarelli, R. Kleiss and C. Verzegnassi, vol. 1, p. 373.

[51] H. Baer, J. Ohnemus, and J. F. Owens, Phys. Rev. D40, 2844 (1989).

[52] B. W. Harris and J. F. Owens, Phys. Rev. D65, 094032 (2002), hep-ph/0102128.

[53] S. D. Ellis, Z. Kunszt, and D. E. Soper, Phys. Rev. D40, 2188 (1989).

[54] S. D. Ellis, Z. Kunszt, and D. E. Soper, Phys. Rev. Lett. 64, 2121 (1990).

[55] Z. Kunszt and D. E. Soper, Phys. Rev. D46, 192 (1992).

[56] W. T. Giele and E. W. N. Glover, Phys. Rev. D46, 1980 (1992).

[57] W. T. Giele, E. W. N. Glover, and D. A. Kosower, Nucl. Phys. B403, 633 (1993), hep-ph/9302225. 
[58] S. Keller and E. Laenen, Phys. Rev. D59, 114004 (1999), hep-ph/9812415.

[59] S. Frixione, Z. Kunszt, and A. Signer, Nucl. Phys. B467, 399 (1996), hep$\mathrm{ph} / 9512328$.

[60] M. L. Mangano, P. Nason, and G. Ridolfi, Nucl. Phys. B373, 295 (1992).

[61] S. Frixione, M. L. Mangano, P. Nason, and G. Ridolfi, Nucl. Phys. B412, 225 (1994), hep-ph/9306337.

[62] B. W. Harris and J. Smith, Nucl. Phys. B452, 109 (1995), hep-ph/9503484.

[63] B. W. Harris and J. F. Owens, Phys. Rev. D54, 2295 (1996), hep-ph/9603305.

[64] S. Dittmaier, Nucl. Phys. B565, 69 (2000), hep-ph/9904440.

[65] S. Catani, M. H. Seymour, and Z. Trocsanyi, Phys. Rev. D55, 6819 (1997), hep-ph/9610553.

[66] S. Catani, S. Dittmaier, and Z. Trocsanyi, Phys. Lett. B500, 149 (2001), hep$\mathrm{ph} / 0011222$.

[67] A. B. Goncharov, Math. Res. Lett. 5, 497 (1998), available at http: //www . math . uiuc.edu/K-theory/0297.

[68] J. M. Borwein, D. M. Bradley, D. J. Broadhurst, and P. Lisonek, Trans. Amer. Math. Soc. 353:3, 907 (2001), math.CA/9910045.

[69] J. A. M. Vermaseren, Int. J. Mod. Phys. A14, 2037 (1999), hep-ph/9806280.

[70] E. Remiddi and J. A. M. Vermaseren, Int. J. Mod. Phys. A15, 725 (2000), hep-ph/9905237.

[71] T. Gehrmann and E. Remiddi, Nucl. Phys. B601, 248 (2001), hep-ph/0008287.

[72] E. Byckling and K. Kajantie, Particle Kinematics (John Wiley and Sons, 1973).

[73] A. Erdélyi et al., Higher Transcendental Functions (Vol. I, McGraw Hill, 1953).

[74] G. Bordes and B. van Eijk, Nucl. Phys. B435, 23 (1995).

[75] M. C. Smith and S. Willenbrock, Phys. Rev. D54, 6696 (1996), hep-ph/9604223.

[76] S. Mrenna and C. P. Yuan, Phys. Lett. B416, 200 (1998), hep-ph/9703224.

[77] T. Stelzer, Z. Sullivan, and S. Willenbrock, Phys. Rev. D56, 5919 (1997), hep$\mathrm{ph} / 9705398$. 
[78] T. Tait and C. P. Yuan, (1997), hep-ph/9710372.

[79] L. J. Bergmann, Ph.D. Thesis, Florida State University (1989), UMI-89-15738.

[80] S. Catani, S. Dittmaier, M. H. Seymour, and Z. Trocsanyi, Nucl. Phys. B627, 189 (2002), hep-ph/0201036.

[81] T. M. P. Tait, Phys. Rev. D61, 034001 (2000), hep-ph/9909352.

[82] A. Belyaev and E. Boos, Phys. Rev. D63, 034012 (2001), hep-ph/0003260.

[83] J. C. Collins and W.-K. Tung, Nucl. Phys. B278, 934 (1986).

[84] T. Gottschalk, Phys. Rev. D23, 56 (1981).

[85] M. Gluck, S. Kretzer, and E. Reya, Phys. Lett. B380, 171 (1996), hep$\mathrm{ph} / 9603304$.

[86] S. Weinzierl, (1999), hep-ph/9903380.

[87] Z. Kunszt, A. Signer, and Z. Trocsanyi, Nucl. Phys. B411, 397 (1994), hep$\mathrm{ph} / 9305239$.

[88] W. Siegel, Phys. Lett. B84, 193 (1979).

[89] W. Siegel, Phys. Lett. B94, 37 (1980).

[90] D. M. Capper, D. R. T. Jones, and P. van Nieuwenhuizen, Nucl. Phys. B167, 479 (1980).

[91] A. Brandenburg, M. Flesch, and P. Uwer, Phys. Rev. D59, 014001 (1999), hep$\mathrm{ph} / 9806306$.

[92] CTEQ, H. L. Lai et al., Eur. Phys. J. C12, 375 (2000), hep-ph/9903282.

[93] T. H. Chang, K. J. F. Gaemers, and W. L. van Neerven, Nucl. Phys. B202, 407 (1982).

[94] J. Blumlein et al., (1996), hep-ph/9609400.

[95] Z. Sullivan and P. M. Nadolsky, eConf C010630, P511 (2001), hep-ph/0111358.

[96] A. D. Martin, R. G. Roberts, W. J. Stirling, and R. S. Thorne, Eur. Phys. J. C14, 133 (2000), hep-ph/9907231.

[97] S. D. Ellis and D. E. Soper, Phys. Rev. D48, 3160 (1993), hep-ph/9305266. 
[98] CDF, F. Abe et al., Phys. Rev. D50, 2966 (1994).

[99] CDF, F. Abe et al., Phys. Rev. Lett. 74, 2626 (1995), hep-ex/9503002.

[100] D0, S. Abachi et al., Phys. Rev. Lett. 74, 2632 (1995), hep-ex/9503003.

[101] CDF, T. Affolder et al., Phys. Rev. D64, 032002 (2001), hep-ex/0101036.

[102] D0, V. M. Abazov et al., Phys. Rev. D67, 012004 (2003), hep-ex/0205019.

[103] Particle Data Group, K. Hagiwara et al., Phys. Rev. D66, 010001 (2002).

[104] N. Kidonakis, E. Laenen, S. Moch, and R. Vogt, Phys. Rev. D64, 114001 (2001), hep-ph/0105041.

[105] E. Laenen, (2003), hep-ph/0308025, talk given at 38th Rencontres de Moriond on QCD and High-Energy Hadronic Interactions, Les Arcs, Savoie, France, 22-29 Mar 2003.

[106] R. Bonciani, S. Catani, M. L. Mangano, and P. Nason, Nucl. Phys. B529, 424 (1998), hep-ph/9801375.

[107] M. Cacciari, S. Frixione, M. L. Mangano, P. Nason, and G. Ridolfi, (2003), hep-ph/0303085.

[108] S. Frixione, P. Nason, and B. R. Webber, JHEP 08, 007 (2003), hep-ph/0305252.

[109] O. Peters, Ph.D. Thesis, Universiteit van Amsterdam (2003).

[110] M. L. Mangano, M. Moretti, F. Piccinini, R. Pittau, and A. D. Polosa, JHEP 07, 001 (2003), hep-ph/0206293.

[111] U. Heintz and M. Narain, DØ Note 2386.

[112] G. Blazey et al., DØ Note 3750.

[113] D0, B. Abbott et al., Nucl. Instrum. Meth. A424, 352 (1999), hep-ex/9805009.

[114] Private communication with Jet Energy Scale group.

[115] M. Kopál, Jet $p_{T}$ resolution with JES v. 4.2, http://www-d0.fnal.gov/ kopal/ files/relate-D0/sumJetRes_0812.2003.ps.

[116] Top Production Group, DØ Note 4116.

[117] S. D. Ellis, R. Kleiss, and W. J. Stirling, Phys. Lett. B154, 435 (1985). 
[118] F. A. Berends, W. T. Giele, H. Kuijf, R. Kleiss, and W. J. Stirling, Phys. Lett. B224, 237 (1989).

[119] F. A. Berends, H. Kuijf, B. Tausk, and W. T. Giele, Nucl. Phys. B357, 32 (1991).

[120] E. Abouzaid and H. Frisch, Phys. Rev. D68, 033014 (2003), hep-ph/0303088.

[121] F. Beaudette and J.-F. Grivaz, DØ Note 4206.

[122] M. Begel et al., DØ Note 3973.

[123] Private communication with EM ID group.

[124] E. Barberis, J.-F. Grivaz, and M. Kado, DØ Note 4246. 


\section{Summary}

This thesis describes both theoretical and experimental research into top quark production. The theoretical part contains a calculation of the single-top quark production cross section at hadron colliders, at Next to Leading Order (NLO) accuracy. The experimental part describes a measurement of the top quark pair production cross section in proton-antiproton collisions, at a center of mass energy of $1.96 \mathrm{TeV}$.

The theoretical work starts with a Leading Order description of the different singletop quark production channels. This description includes all Feynman diagrams that have the same final state as the one where the top quark decays to a lepton, neutrino and a bottom quark. Only a small subset of the diagrams actually contains a top quark. It is shown that in each of the channels the top quark is clearly seen in the distribution of the invariant mass of the lepton-neutrino-bottom combination. In addition, the validity of the narrow width approximation for these channels is studied.

The next step in the calculation, a NLO description, requires the cancellation of infrared divergences. The most advanced method to handle this in general is the dipole method. The extension of this method to massive fermions is developed in this thesis.

In the final part of the theoretical work expressions for the virtual and radiative contributions needed for the NLO calculation are derived. These are then used, together with the dipole formalism, to implement a NLO Monte Carlo program for single-top production. In addition, NLO programs were implemented that treat the infrared divergences using the method of phase space slicing. All methods are shown to give the same distributions for several kinematical variables, and in addition total and differential cross sections are calculated for the Tevatron and LHC colliders at different energies.

The experimental work was done on data collected by the D $\varnothing$ experiment at the Tevatron collider at Fermilab. The Tevatron is an accelerator that collides protons and antiprotons at a center of mass energy of $1.96 \mathrm{TeV}$. The large D $\varnothing$ detector surrounds one of the collision points. It consists of several subdetectors, of which the calorimeter is the most important for the analysis described in this thesis.

Due to the very high collision rate, a trigger system (consisting of three levels) is used to select the most interesting events. A trigger was designed that selects events 
that have an electron and jet activity in the calorimeter. Efficiencies for the different components of the trigger and the total efficiency for the events we are interested in are derived.

The experimental analysis addresses the top pair production process in the case where one of the $W$ 's (from the top) decays leptonically, and the other decays to hadrons. Such a decay mode results in an electron, missing transverse energy $\left(\mathbb{E}_{T}\right)$ and four jets in the final state. To reduce the huge background from QCD processes in this decay channel, an electron identification that gives a good separation between real and fake electrons is needed. Several variables give some discrimination between these real and fake electrons. A likelihood method is developed that combines the information in six of these variables to get an optimal separation.

The cross section measurement was performed on a data sample corresponding to an integrated luminosity of $92 \mathrm{pb}^{-1}$. The two important backgrounds are the already mentioned QCD background, and the background from $W+$ jets events, in which the $W$ decays to an electron and a neutrino. Both backgrounds are estimated from data. The QCD background is minimized by requiring a good quality electron in addition to $E_{T}$. The remaining QCD background is estimated by studying the behavior of the event sample under electron identification cuts. The $W+$ jets background is estimated using the Berends scaling rule, which predicts the number of $W$ plus four jet events from the number of $W$ 's observed with one, two and three jets. After these backgrounds have been estimated, a final set of topological cuts is applied to improve the signal significance. This results in a $t \bar{t}$ cross section measurement in this channel of

$$
6.2{ }_{-4.0}^{+4.9} \text { (stat) }{ }_{-2.7}^{+2.8}(\text { syst }) \quad \mathrm{pb} .
$$

Combining this result with measurements in different decay channels gives a combined $t \bar{t}$ cross section measurement of

$$
8.1{ }_{-2.0}^{+2.2} \text { (stat) }{ }_{-1.4}^{+1.6}(\text { syst }) \pm 0.8(\text { lumi }) \mathrm{pb}
$$

consistent with the Standard Model prediction. 


\section{Samenvatting}

Dit proefschrift beschrijft zowel theoretisch als experimenteel onderzoek naar top quark productie. Het theoretisch gedeelte bevat een berekening van de werkzame doorsnede van single-top quark productie in hadron botsingen, op Next to Leading Order (NLO) nauwkeurigheid. Het experimentele gedeelte beschrijft een meting van de top paar productie werkzame doorsnede in proton-antiproton botsingen met een zwaartepuntsenergie van $1.96 \mathrm{TeV}$.

Het theoretisch werk begint met een Leading Order beschrijving van de verschillende single-top quark productie kanalen. Deze beschrijving bevat alle Feynman diagrammen die dezelfde eindtoestand hebben als die waar het top quark vervalt naar een lepton, een neutrino en een bottom quark. Slechts een klein deel van deze diagrammen bevat daadwerkelijk een top quark. Er wordt aangetoond dat in elk van de kanalen het top quark duidelijk te zien is in de distributie van de invariante massa van de lepton-neutrino-bottom combinatie. Ook wordt de geldigheid van de "narrow width" benadering voor deze kanalen bestudeerd.

De volgende stap in de berekening, een NLO beschrijving, vereist het tegen elkaar wegvallen van de verschillende infrarood divergenties. De meest geavanceerde methode om dit in het algemene geval te doen is de dipool methode. De uitbereiding van deze methode naar massieve fermionen is in dit proefschrift ontwikkeld.

In het laatste deel van het theoretisch werk worden uitdrukkingen voor de virtuele en stralings bijdragen afgeleid, die nodig zijn voor de NLO berekening. Deze worden tezamen met het dipool formalisme gebruikt om een NLO Monte Carlo programma voor single-top produktie te implementeren. Bovendien zijn er NLO programmas geïmplementeerd die de infrarood divergenties behandelen met behulp van de phase space slicing methode. Alle methoden geven dezelfde distributies voor diverse kinematische variabelen. Verder zijn er totale en differentiële werkzame doorsnedes berekend voor de Tevatron en LHC versnellers bij verschillende energieën.

Het experimentele werk is verricht met data verzameld door het $D \varnothing$ experiment gelegen aan de Tevatron versneller op Fermilab. De Tevatron is een versneller die protonen en antiprotonen laat botsen met een zwaartepuntsenergie van $1.96 \mathrm{TeV}$. De DØ detector omringt één van de interactie punten van de versneller. De detector 
bestaat uit diverse subdetectoren, van welke de calorimeter het belangrijkst is voor de analyse die in dit proefschrift beschreven wordt.

Omdat de botsings frequentie zeer hoog is, wordt een trigger systeem (bestaande uit drie niveaus) gebruikt om de meest interessante events te selecteren. Er is een trigger ontworpen die events selecteert die een electron en jet activiteit in de calorimeter hebben. De efficiënties voor de verschillende componenten van de trigger en de totale efficiëntie voor de events waar we in zijn geïnteresseerd worden afgeleid.

De experimentele analyse bekijkt het top paar productie process in het geval waar één van de $W$ 's (van de top) leptonisch vervalt, en de ander naar hadronen. Dit verval resulteert in een electron, ontbrekende transversale energie $\left(E_{T}\right)$ en vier jets in de eindtoestand. Om de enorme achtergrond van QCD processen in dit vervalskanaal te reduceren, is een electron identificatie nodig die een goede scheiding tussen echte en misgeïndentificeerde electronen geeft. Verscheidene variabelen geven enige discriminatie tussen echte en misgeïndentificeerde electronen. Een waarschijnlijkheids methode is ontwikkeld die de informatie in zes van deze variabelen combineert om een optimale scheiding te krijgen.

De werkzame doorsnede meting is uitgevoerd op een data set overeenkomend met een geïntegreerde luminositeit van $92 \mathrm{pb}^{-1}$. De twee belangrijke achtergronden zijn de al genoemde QCD achtergrond, en de achtergrond van $W+$ jets events, in welke de $W$ vervalt naar een electron en een neutrino. Beide achtergronden worden afgeschat met behulp van data. De QCD achtergrond wordt geminimaliseerd door een goede kwaliteit electron en $E_{T}$ te eisen. De overgebleven QCD achtergrond wordt geschat door het gedrag van de events te bestuderen onder variatie van de electron identificatie snedes. De $W+$ jets achtergrond wordt geschat door gebruik te maken van de Berends schalings regel, die het aantal $W$ plus vier jets events voorspelt uit het aantal geobserveerde $W$ 's met een, twee en drie jets. Nadat deze achtergronden zijn afgeschat, wordt een set topologische snedes gebruikt om de signaal significantie te verbeteren. Dit resulteert in een $t \bar{t}$ werkzame doorsnede meting in dit kanaal van

$$
6.2{ }_{-4.0}^{+4.9} \text { (stat) }{ }_{-2.7}^{+2.8}(\text { syst }) \quad \mathrm{pb}
$$

Indien we dit resultaat combineren met metingen in andere vervalskanalen, krijgen we een gecombineerde $t \bar{t}$ werkzame doorsnede meting van

$$
8.1{ }_{-2.0}^{+2.2}(\text { stat }){ }_{-1.4}^{+1.6}(\text { syst }) \pm 0.8(\text { lumi }) \mathrm{pb},
$$

wat consistent is met de Standaard Model voorspelling. 


\section{Acknowledgements}

Let's start at the beginning. I would like to thank Frits Berends for putting forward the option of doing a combined theoretical/experimental $\mathrm{PhD}$ thesis, and for getting me in contact with Eric Laenen and Frank Linde. I thank Frank and Eric for giving me this opportunity.

In the first two years, I worked in the NIKHEF theory group. There I worked together with Stefan Weinzierl, who, with endless patience, explained me how to do the calculations. The daily support from Eric in this period was also highly appreciated, and so were the additional explanations by Sven Moch. My fellow PhD students Tim Eynck and Tino Nyawelo were great fun to talk to and hang out with.

I would also like to mention our friends Peter Overduin and Aart Draijer here. Although they have nothing to do with physics, they were a great help, which included offering me and my wife (and our four suitcases) a place to stay the four weeks that we were homeless before we left for Chicago.

Arriving at Fermilab, it was great to be supported by all the PhD students from our group. I would like to especially mention Paul Balm and Onne Peters, who got me up to speed in the complicated world of $D \varnothing$. Onne was also a very good neighbor, with who we spent a lot of evenings. We also have a lot of nice memories from Friday night dinner+drinks+movies together with Martijn Mulders, Michiel Sanders and whoever wanted to come along.

During my 18 month stay at Fermilab, I recieved lots of good advice from Marcel Demarteau. Although he was very busy, he always found time for friday afternoon visits to our office. After I decided to switch analysis subject, Ia Iashvili helped me get started with the top pair cross section analysis. While doing this analysis, I recieved lots of good advice and help from Daniel Whiteson and Markus Klute, two of my fellow analyzers.

In the second summer at Fermilab, besides work, we spent most of our time on Lake Michigan, racing on Rainbow's End. A special thanks go to her skipper, Steven Fink, and fellow crew members Darren Beck and Amy Guajardo for making this the best summer ever. And let's not forget the shore crew, Kristina and Lisa, for the hospitality and the cookies!

All good things have to end, so in December 2002 we came back to the Netherlands. 
There I again kept Onne and Paul from their work with my endless questions. When it came to the thesis writing stage, Paul de Jong did a great job by quickly proofreading endless iterations of the chapters of my thesis.

Through all this, the support of my parents, brother and wife was unconditional. 